\title{
Measurement of the Forward-Backward Asymmetry in Top-Antitop Quark Events in the Lepton+Jets Channel at DØ
}

\author{
by \\ Douglas A. Orbaker \\ Submitted in Partial Fulfillment \\ of the Requirements for the Degree \\ Doctor of Philosophy
}

Supervised by

Regina Demina

Department of Physics and Astronomy

Arts, Sciences and Engineering

School of Arts and Sciences

\author{
University of Rochester \\ Rochester, New York
}


To my parents.

You might not have any idea what I do, but you support me nonetheless. 


\section{Curriculum Vitae}

The author

attended Amherst College from 2000 to 2004 and graduated with a Bachelor of Arts in 2004. He came to the University of Rochester in Fall 2006 and began graduate studies in Physics. In 2008 he successfully completed the preliminary examination and earned a Master of Arts. He began research in the field of high energy physics under the direction of Professor R. Demina in 2007, which has culminated in the work presented in this dissertation. 


\section{Acknowledgments}

First and foremost, I would like to thank Regina for giving me the opportunity to work on this measurement and for taking me on as a graduate student.

I am also extremely grateful for all of the help and countless hours of discussion over Skype offered by Amnon. I do not think this analysis would have been completed without your support.

I would like to thank my friends and colleagues at Fermilab for many interesting conversations over lunch and coffee at DØ. There are many, but Tim, Yvonne, Louise, Kostas and Carrie stick out the most in my memory. If it wasn't for you guys, I would have never known about Two Brothers.

I would like to thank my friends at Rochester, particularly my brothers in arms Burt and Dan. Thanks to Sergey for welcoming me to the group with a warm smile and open arms.

I would like to thank my parents and my sister, Jen, for all of their support and excitement about my research. Along with my family, I would like to thank Sarah's parents and her sisters, Abby and Becca.

I would also like to thank cnn.com, for helping me keep things in perspective: "Fermilab scientists also recently announced a possible discovery of something called 'forward-backward top quark asymmetry.' That doesn't sound as sexy as 'new particle potentially discovered', but physicists are enthusiastic about it, too." - "Is it a new particle, or just a fluke?", Elizabeth Landau, cnn.com, April 8, 2011

Last but not least, I would like to thank my wife Sarah for all of her support during my graduate career. Her intelligence, drive and sense of humor have inspired me and kept me going. 


\section{Abstract}

We present a measurement of forward-backward asymmetries in top-antitop quark pairs produced in proton-antiproton collisions decaying via the lepton+jets channel. Using data recorded by the $\mathrm{D} \varnothing$ experiment at the Fermilab Tevatron collider and corresponding to an integrated luminosity of $5.4 \mathrm{fb}^{-1}$, we measure the forward-backward asymmetry in top-antitop quark events to be $(9.2 \pm 3.7) \%$, after background processes have been subtracted. After correcting for the effects of acceptance and detector reconstruction, we measure an asymmetry of $(19.6 \pm 6.5) \%$. In addition, we measure an acceptance-corrected asymmetry based on the lepton from top-antitop quark decay of $(15.2 \pm 4.0) \%$. We compare these results to predictions from the MC@NLO next-to-leading-order QCD simulation. 


\section{Table of Contents}

Curriculum Vitae iii

Acknowledgments $\quad$ iv

$\begin{array}{lll}\text { Abstract } & \text { V }\end{array}$

List of Tables $\quad$ ix

List of Figures xii

List of Abbreviations $\quad$ xviii

1 Foreword 1

2 Introduction $\quad 2$

2.1 Quick and hole-filled history of particle physics . . . . . . . . 2

2.2 The standard model . . . . . . . . . . . . . . . . . 3

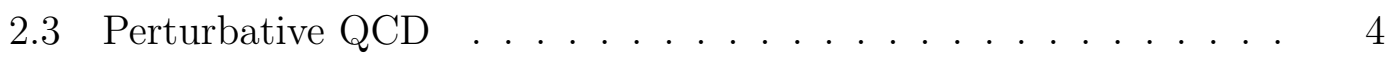

2.4 The top quark . . . . . . . . . . . . . . . 5

2.5 Top quark production at the Tevatron . . . . . . . . . . 6

2.6 Top quark decay . . . . . . . . . . . . . . . . . . . 7

2.7 Backgrounds to top quark decay . . . . . . . . . . . . . . . 9

2.8 Observable quantities . . . . . . . . . . . . . . . . . . . 10

2.9 Definition of forward-backward asymmetry . . . . . . . . . . . . 12 
2.10 Forward-backward asymmetry in $t \bar{t}$ production . . . . . . . . . . . 14

2.11 Asymmetry beyond the standard model . . . . . . . . . . . . . 18

2.12 Connecting theory to measurement . . . . . . . . . . . . . 19

2.13 An analogy ......................... 21

3 Experimental Apparatus $\quad 23$

3.1 Accelerator ........................ 23

3.2 DØ Detector . . . . . . . . . . . . . . . . 26

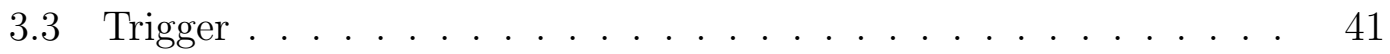

4 Object Identification $\quad 45$

4.1 Track Identification . . . . . . . . . . . . . . . . . 45

4.2 Primary Vertex Reconstruction . . . . . . . . . . . . . . . 47

4.3 Electrons. . . . . . . . . . . . . . . . . . 48

4.4 Muons . . . . . . . . . . . . . . . . . . . 52

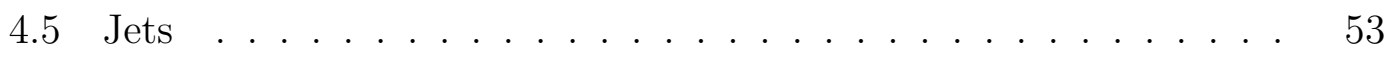

$4.6 \quad$ b Jets. . . . . . . . . . . . . . . . . . . . . . . 59

4.7 Missing Transverse Energy . . . . . . . . . . . . . . . . 62

5 Data and Simulation $\quad 64$

5.1 Data Sample.................... 64

5.2 Event Simulation . . . . . . . . . . . . . . . 65

5.3 Event Weights.................... 69

6 Selection and Reconstruction $\quad 71$

6.1 Event Selection . . . . . . . . . . . . . . . . 71

6.2 Matrix Method for Multijet Background Determination . . . . . . 74

6.3 Constrained Kinematic Fit . . . . . . . . . . . . . . . . . . 75 
7 Analysis Technique and Results $\quad 79$

7.1 Predicting reconstruction-level asymmetries . . . . . . . . . 79

7.2 Reconstruction-level measurements . . . . . . . . . . . . . . 79

7.3 Production-level measurements . . . . . . . . . . . . . . . . . 90

7.4 Ensemble testing of procedures . . . . . . . . . . . . . 103

7.5 Crosschecks ......................... 111

7.6 Systematic Uncertainties . . . . . . . . . . . . . . . . 119

8 Conclusion $\quad 124$

8.1 Projections for the Future . . . . . . . . . . . . . 125

$\begin{array}{ll}\text { Bibliography } & 126\end{array}$

A Derivation of statistical uncertainty for weighted events 136

$\begin{array}{ll}\text { B Dilution } & 137\end{array}$

$\begin{array}{ll}\text { C Template fits } & 141\end{array}$

$\begin{array}{ll}\text { D Complete systematics } & 144\end{array}$ 


\section{List of Tables}

2.1 Table of particles in the standard model. . . . . . . . . . . . . 4

5.1 Integrated luminosity analyzed in $e+$ jets final states in Run IIa. $\quad 65$

5.2 Integrated luminosity analyzed in $\mu+$ jets final states in Run IIa. $\quad 65$

5.3 Integrated luminosity analyzed in $e+$ jets and $\mu+$ jets final states in Run IIb. . . . . . . . . . . . . . . . . . . . . . . . . . . . 66

6.1 Efficiencies for isolated leptons and mis-identified jets to pass the tight selection criteria. The uncertainties include both systematic and statistical contributions. . . . . . . . . . . . . . 75

6.2 Table of potential jet-parton assignments for the reconstructed top quarks for events with one $b$ tag. . . . . . . . . . . 76

6.3 Table of potential jet-parton assignments for the reconstructed top quarks for events with two $b$ tags. . . . . . . . . . . . . 77

7.1 Predictions from MC@NLO. The quoted uncertainties are statistical, from the size of the MC sample. . . . . . . . . . . . . . 80

7.2 Sample sizes and $A_{\mathrm{FB}}$ fit results. Only statistical uncertainties are included. . . . . . . . . . . . . . . . . . . 87

7.3 Result of fit for sample composition and lepton-based reconstructionlevel $A_{\mathrm{FB}}^{l}$. Only statistical uncertainties are included. . . . . . . . 88

7.4 Relative selection efficiency between bins in $\Delta y$, shown so that the trace of $\mathrm{A}$ is equal to $4 . \Delta y$ increases as the entries go from left to right. . . . . . . . . . . . . . . . . . 92 
7.5 Results using simultaneous unfolding of $\Delta y$ and $m_{t \bar{t}}$ with comparison of CDF results. . . . . . . . . . . . . . . . . . . . . . . 93

7.6 Experimental resolution and the fraction of problematic events for different observables. The cosmic ray resolutions in data are used as a crosscheck for the simulated resolution. $y_{t \text {,had }}$ refers to the rapidity of the hadronically-decaying top quark. . . . . . . . . . . 98

7.7 Predicted and observed lepton-based asymmetries. . . . . . . . . 103

7.8 Number of signal and background events for each type of process included in ensemble testing. . . . . . . . . . . . . . . . . . . . 104

$7.9 A_{\mathrm{FB}}$ and $A_{\mathrm{FB}}^{l}$ shown for events with different numbers of $b$-tagged

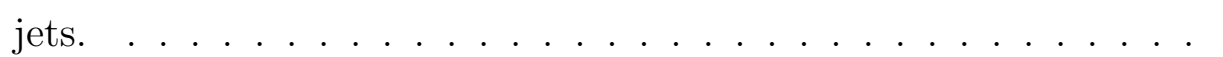

7.10 Fitted sample compositions and $\Delta y$-based asymmetries for $t \bar{t}$ and $W+$ jets, with the simulated $W+$ jets asymmetry. . . . . . . . 116

7.11 Fitted sample compositions and lepton-based asymmetries for $t \bar{t}$ and $W+$ jets, with the simulated $W+$ jets asymmetry. The number of data events $\left(N_{\mathrm{t}}\right.$ and $\left.N_{\mathrm{l}-\mathrm{t}}\right)$ is the same as in Table 7.10 . . . . . 116

7.12 Fitted sample compositions and $A_{\mathrm{FB}}$ by magnet polarity. . . . . . 117

7.13 Fitted sample compositions and $A_{\mathrm{FB}}^{l}$ by magnet polarity. The number of data events $\left(N_{\mathrm{t}}\right.$ and $\left.N_{\mathrm{l}-\mathrm{t}}\right)$ and the fitted sample composition $\left(N_{t \bar{t}}, N_{W}\right.$, and $\left.N_{\mathrm{MJ}}\right)$ are the same as in Table $7.10 . \ldots 118$

$7.14 A_{\mathrm{FB}}$ by toroid polarity and channel. . . . . . . . . . . . . . 118

$7.15 A_{\mathrm{FB}}^{l}$ by toroid polarity and channel. . . . . . . . . . . . . . 118

7.16 Systematic uncertainties on the $A_{\mathrm{FB}}$. Only uncertainties above $0.1 \%$ are listed. . . . . . . . . . . . . . . 122

7.17 Systematic uncertainties on the $A_{\mathrm{FB}}^{l}$. Only uncertainties above $0.1 \%$ are listed. . . . . . . . . . . . . . . 123

B.1 Dilution Parameters. . . . . . . . . . . . . . . . . 138

B.2 Comparison between asymmetries predicted with Equation B.5 (center column) and the full detector simulation (right column). . . . 140 
D.1 Absolute systematic uncertainties on $A_{\mathrm{FB}}^{\mathrm{pred}}$. The 2 nd and 3rd columns list the effect of each systematic variation on $A_{\mathrm{FB}}^{\mathrm{pred}}$, and the 4 th and 5th columns list the resulting up and down uncertainties. . . . . 144

D.2 Absolute systematic uncertainties on $A_{\mathrm{FB}}^{\text {lep,pred }}$. The 2 nd and $3 \mathrm{rd}$ columns list the effect of each systematic variation on $A_{\mathrm{FB}}^{\text {lep,pred }}$, and the 4 th and 5 th columns list the resulting up and down uncertain-

D.3 Absolute systematic uncertainties on the observed $A_{\mathrm{FB}}$. The 2 nd and 3rd columns list the effect of each systematic variation on $A_{\mathrm{FB}}$, and the 4th and 5th columns list the resulting up and down uncertainties. . . . . . . . . . . . . . . . . .

D.4 Absolute systematic uncertainties on the observed $A_{\mathrm{FB}}^{l}$. The 2 nd and 3rd columns list the effect of each systematic variation on $A_{\mathrm{FB}}^{l}$, and the 4th and 5th columns list the resulting up and down uncer-

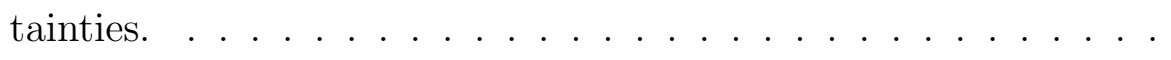

D.5 Absolute systematic uncertainties on the $50 \rightarrow 26$ unfolded $A_{\mathrm{FB}}$. The 2nd and 3rd columns list the effect of each systematic variation on the unfolded $A_{\mathrm{FB}}$, and the 4 th and 5 th columns list the resulting up and down uncertainties. . . . . . . . . . . . . 148

D.6 Absolute systematic uncertainties on the unfolded $A_{\mathrm{FB}}^{l}$. The 2nd and 3rd columns list the effect of each systematic variation on the unfolded $A_{\mathrm{FB}}^{l}$, and the 4 th and 5 th columns list the resulting up and down uncertainties. . . . . . . . . . . . . . . . . . . 149 


\section{List of Figures}

2.1 Feynman diagrams for $t \bar{t}$ production at the Tevatron, with $q \bar{q} \rightarrow t \bar{t}$ shown on the left and $g g \rightarrow t \bar{t}$ shown on the right. . . . . . . 7

2.2 Feynman diagrams of single top production at the Tevatron, with $q g \rightarrow \bar{q}^{\prime} b \bar{t}$ ( $t$ channel, left) and $q \bar{q}^{\prime} \rightarrow t \bar{b}$ ( $s$ channel, right). . . . . 7

2.3 Example Feynman diagrams of the $e+$ jets channel (left) and the $\mu+$ jets channel (right) for $t \bar{t}$ decay. . . . . . . . . . . . . . 8

2.4 Example Feynman diagram of $W+$ jets background for $t \bar{t}$ decay. $\quad$. 9

2.5 Example Feynman diagram of MJ background for $t \bar{t}$ decay. . . 10

2.6 Examples of forward (left) and backward (right) events. . . . . . 13

2.7 Example Feynman diagrams that interfere to produce $A_{\mathrm{FB}}$ in $t \bar{t}$ production. . . . . . . . . . . . . . . . 16

2.8 Feynman diagrams for $t \bar{t}$ production via massive color octets (left) and $t$-channel $Z^{\prime}$ exchange (right). . . . . . . . . . . . . . . . . 19

3.1 The sequence of accelerators that constitute the accelerator chain at Fermilab. . . . . . . . . . . . . . . . . . . . . . 24

3.2 Cross section of the $\mathrm{D} \varnothing$ detector as viewed from inside the Tevatron ring, facing outward. . . . . . . . . . . . . . . 27

3.3 Diagram of the SMT. . . . . . . . . . . . . . . . . . . 28

3.4 Diagram of the tracking system, with focus on the CFT. . . . . . 29

3.5 Diagram of the solenoid and toroid magnet fields used in the $\mathrm{D} \varnothing$ detector. . . . . . . . . . . . . . . . 31 
3.6 a) Cross section of the triangular scintillator used in the preshower, with the wavelength-shifting fiber shown in the center. b) Geometry of scintillator stacking in the CPS. c) Geometry of scintillator stacking in the FPS. . . . . . . . . . . . . . .

3.7 Diagram of an example wedge from the FPS showing the stereo angle of the fibers. . . . . . . . . . . . . . . . 32

3.8 Cut-away view of the $\mathrm{D} \varnothing$ calorimeter. . . . . . . . . . . . . 34

3.9 Cross section of the calorimeters showing $\eta$. . . . . . . . . 35

3.10 Schematic diagram of an individual calorimeter cell. . . . . . . . . 35

3.11 Simulated energy deposition spectrum of a shower initiated by a 45 $\mathrm{GeV}$ electron, overlaid with the different sections of the calorimeter. 36

3.12 View of the drift tubes (top) and scintillation counters (bottom) in the muon system. . . . . . . . . . . . . . 37

3.13 Side view of the PDTs used in the central muon system. . . . . . 39

3.14 Cross section of the eight cells making up an MDT. . . . . . . . . 39

3.15 Picture of the scintillator panels in layer $\mathrm{C}$ of the forward muon detector. . . . . . . . . . . . . . . . . 40 40

3.16 Diagram of the luminosity monitor, with the 24 PMTs displayed at red circles. . . . . . . . . . . . . . . . . . 42

3.17 Cross sections for different processes at different colliding energies. The discontinuities occur because of the difference between the colliding particles at the Tevatron $(p \bar{p})$ and the LHC (pp). . . . . . 43

3.18 Diagram of the trigger framework. . . . . . . . . . . . . . . . 44

3.19 Example of trigger rates and luminosity at $\mathrm{D} \varnothing$ taken from one round of Tevatron collisions. . . . . . . . . . . . . . . . 44

4.1 An example of a track from a charge particle passing through the CFT detector, reconstructed from clusters of hits. . . . . . . . 46

4.2 Diagram of a hard-scattered parton decaying into a jet. . . . . . 57

4.3 Diagram describing the MPF method. . . . . . . . . . . . 58

4.4 Plot of the absolute response for photon+jet events. . . . . . . . 59 
4.5 Plot of the fractional JES uncertainty. . . . . . . . . . . 60

4.6 The neural net outputs for jets from simulated $b$ hadrons and light hadrons (left). Comparison of the efficiency and mis-tag rates for the NN algorithm and the JLIP method (right). . . . . . . . . .

5.1 Integrated luminosity for Run II of DØ. This analysis uses data recorded up to the red line. . . . . . . . . . . . 66

5.2 Example parton distributions functions measured at HERA. . . . 67

5.3 Production asymmetry (left) and top quark tranverse momentum (right) from NLO predictions, HERWIG, and MC@NLO. HERWIG does not predict any asymmetry, while NLO predictions and MC@NLO do................................. 68

6.1 Which jets originate from which quarks? D $\varnothing$ event display of toplike $e+$ jets event (left) and rendition of $t \bar{t}$ decay to lepton+jets (right) . . . . . . . . . . . . . . 76

$6.2 \chi^{2}$ distribution from the kinematic fit for $t \bar{t}$ signal events. . . . . . 78

7.1 Comparison of data and simulation for the four variables used to construct the discriminant. . . . . . . . . . . 86

7.2 Distributions in the discriminant for $\Delta y<0$ (a) and $\Delta y>0$ (b). 86

7.3 The $\Delta y$ distribution for the data, shown with the fitted sample composition. ................. 86

7.4 The $q_{l} y_{l}$ distribution for data, shown with the fitted sample composition. . . . . . . . . . . . . . . 88

7.5 Differential asymmetry measurements with respect to $m_{t \bar{t}}$ (left) and $|\Delta y|$ (right). Results from the CDF collaboration are included in the left plot. . . . . . . . . . . . . . . . . 89

7.6 The 4 by 4 migration matrix used in the simplest form of unfolding. 91

7.7 Migration matrix for different $\Delta y$ and $m_{t \bar{t}}$ regions. . . . . . . . 93

7.8 Data and simulation for the fit to the discriminant (left) and the fit to $\Delta y$ with the template unfolding technique (right) . . . . . . 96 
7.9 Experimental resolution for different observables. $y_{\text {th }}$ refers to the rapidity of the hadronically-decaying top quark. $y_{1}$ refers to the rapidity of the lepton. . . . . . . . . . . . . . . . . . . . 97

7.10 Left: Migration matrix used for regularization with 50 bins for reconstructed $\Delta y$ and 26 bins for the produced $\Delta y$. Right: Selection efficiency as a function of $\Delta y . \ldots \ldots$. . . . . . . . . . . . 98

7.11 Example of an L-curve used to choose the regularization strength. The standard likelihood from matrix inversion is shown on the $\mathrm{x}$ axis. The likelihood from the regularization term is shown on the yaxis. The size of the $\tau$ parameter and the amount of regularization increase as the points move toward the right of the plot. . . . . . 100

7.12 Example simulated data unfolded with different regularization strengths: under regularized (left), more-or-less optimally regularized (center) and over regularized (right). . . . . . . . . . . . . 100

7.13 Effect of different regularization strengths on the statistical fluctuation and the bias of the asymmetry for multiple input distributions. The optimized parameter is shown with an arrow. . . . . . . 100

7.14 Bias for different $\Delta y$ distributions. For positive asymmetries, the bias in general causes the unfolded asymmetry to be smaller. The solid line is fit to the difference between the unfolded and true asymmetries. The dotted lines represent the systematic uncertainty on the bias correction. . . . . . . . . . . . . . . 101

7.15 Comparison of $\Delta y$ distribution from data and the regularized $\Delta y$ distribution after "folding" it back to the reconstruction-level. . . 102

7.16 Selection efficiency as a function of $q_{l} y_{l}$. Because of the sharp drops at $\left|y_{l}\right|=1.5$, an additional selection criterion of $\left|y_{l}\right|<1.5$ is included for $A_{\mathrm{FB}}^{l} \ldots \ldots \ldots \ldots$. . . . . . . . . . . . . . 103

7.17 The fitted number of events minus the actual number of events in each PDS for $t \bar{t}$ (top, left), $W+$ jets (top, right) and MJ (bottom).

7.18 The fitted asymmetry minus the true asymmetry for each PDS for $A_{\mathrm{FB}}$ (left) and $A_{\mathrm{FB}}^{l}$ (right). . . . . . . . . . 106 
7.19 Correlation plots for unfolded $A_{\mathrm{FB}}$ versus reconstruction-level $A_{\mathrm{FB}}$ (top, left), unfolded $A_{\mathrm{FB}}^{l}$ versus reconstruction-level $A_{\mathrm{FB}}^{l}$ (top, right), reconstruction level $A_{\mathrm{FB}}^{l}$ versus reconstruction level $A_{\mathrm{FB}}$ (bottom, left) and unfolded $A_{\mathrm{FB}}^{l}$ versus unfolded $A_{\mathrm{FB}}$ (bottom, right). The red lines represent the values measured from data. . . . . . . . . 107

7.20 Correlation plots with confidence contour lines for different asymmetries. The plots on the left compare reconstruction-level asymmetries and the plots on the right compare unfolded asymmetries. Events in the bottom plots are weighted to change the production level asymmetries. The red point represents values measured from data. Systematic uncertainties are not included. . . . . . . . . . . 110

7.21 Correlation plots with standard error contour lines for different asymmetries. The plot on the left compares reconstruction-level asymmetries and the plot on the right compares unfolded asymmetries. The red point represents values measured from data. Systematic uncertainties are not included. . . . . . . . . . . . . 110

7.22 The templates used in the fit for the $W+$ jets $\Delta y$-based asymmetry. Shown fully (top), and without the $\operatorname{sgn}(\Delta y)$ bins below (bottom).

7.23 The templates used in the fit for the $W+$ jets $q_{l} y_{l}$-based asymmetry. Shown fully (top), and without the $\operatorname{sgn}\left(q_{l} y_{l}\right)$ bins (bottom). . . . . 114

7.24 Data versus simulation for the $q_{l} y_{l}$ (left) and $\Delta y$ (right) distributions for events with 0 b-tagged jets. . . . . . . . . . . 114

7.25 Data versus simulation for the $q_{l} y_{l}$ (left) and $\Delta y$ (right) distributions for events with 1 b-tagged jet. . . . . . . . . . 115

7.26 Data versus simulation for the $q_{l} y_{l}$ (left) and $\Delta y$ (right) distributions for events with 2 or more $b$-tagged jets. . . . . . . . .

8.1 Correlation plots with sigma contour lines for different asymmetries. The plots on the left compare reconstruction-level asymmetries and the plots on the right compare unfolded asymmetries. These plots are a projection for the future with the amount of data doubling and the measured asymmetries staying the same. The red lines represent the current values measured from data. . . . . . . 125 
B.1 Dilution for $A_{\mathrm{FB}}$ using reconstructed $t \bar{t}$ events from MC@NLO. The dilution curves are shown separately for the fully reconstructed $\Delta y$ (left), and for the signed lepton rapidty, $\left|q_{l} y_{l}\right|$ (right). . . . . 138

B.2 Fitted functions for for $A_{\mathrm{FB}}(\Delta y)$ with different levels of selection. $A_{\mathrm{FB}}(\Delta y)$ before selection (top, left), $A_{\mathrm{FB}}(\Delta y)$ after the full selection (top, right) and $A_{\mathrm{FB}}(\Delta y)$ after parameterized selection with "particle" jets (bottom). . . . . . . . . . . . . . . . 139

C.1 Run IIa template fits. The left plots show the distributions of the input variables used in the likelihood discriminant in simulated $t \bar{t}$ events (red curve) and $W+$ jets events (dashed blue curve). The right plots show the logarithms of the ratios of these distributions (points and histogram), and the fit to them (curve). Overflows are shown in the extreme bins. . . . . . . . . . . . . . . 142

C.2 Run IIb template fits. The left plots show the distributions of the input variables used in the likelihood discriminant in simulated $t \bar{t}$ events (red curve) and $W+$ jets events (dashed blue curve). The right plots show the logarithms of the ratios of these distributions (points and histogram), and the fit to them (curve). Overflows are shown in the extreme bins. . . . . . . . . . . . . . . 143 
xviii

\section{List of Abbreviations}

$\begin{array}{ll}\text { ALPGEN } & \text { ALPGEN MC simulation } \\ \text { AA } & \text { Alternative Algorithm } \\ \text { ADC } & \text { Analog to Digital Converter } \\ \text { BSM } & \text { Beyond the Standard Model } \\ \text { CC } & \text { Central Calorimeter } \\ \text { CDF } & \text { Collider Detector at Fermilab } \\ \text { CFT } & \text { Central Fiber Tracker } \\ \text { CH } & \text { Coarse Hadronic Calorimeter } \\ \text { CPS } & \text { Central Preshower Detector } \\ \text { CSIP } & \text { Counting Signed Impact Parameters } \\ \text { EC } & \text { End Calorimeter } \\ \text { EM } & \text { Electromagnetic Calorimeter } \\ \text { FCNC } & \text { Flavor Changing Neutral Currents } \\ \text { FH } & \text { Fine Hadronic Calorimeter } \\ \text { FNAL } & \text { Fermi National Accelerator Laboratory } \\ \text { FPS } & \text { Forward Preshower Detector } \\ \text { FSR } & \text { Final State Radiation } \\ \text { HITFIT } & \text { Kinematic fitting program } \\ \text { HTF } & \text { Histogram Track Finder } \\ \text { ICD } & \text { Inter Cryostat Detector } \\ \text { ISR } & \text { Initial State Radiation } \\ \text { JER } & \text { Jet Energy Resolution } \\ \text { JES } & \text { Jet Energy Scale } \\ \text { JLIP } & \text { Jet Lifetime Probability } \\ \text { L-T } & \text { Loose Minus Tight } \\ \text { LD } & \text { Likelihood Discriminant } \\ & \end{array}$


LHC Large Hadron Collider

LO Leading Order

MC Monte Carlo

MI Main Injector

MIP Minimum Ionizing Particle

MJ Multijet

MTC Muon Tracking in Calorimeter

NLO Next to Leading Order

NN Neural Net

NNLL Next to Next to Leading Logarithm

NNLO Next to Next to Leading Order

PDF Parton Distribution Function

PDS Pseudo Data Set

pQCD perturbative Quantum Chromodynamics

QCD Quantum Chromodynamics

QED Quantum Electrodynamics

ROOT Program for physics analysis

SLT Soft Lepton Tagger

SM Standard Model of particle physics

SMT Silicon Microstrip Tracker

SVT Secondary Vertex Tagger

TRF Tag Rate Function

D $\quad$ The D $\varnothing$ Detector or The D $\varnothing$ Collaboration

$E_{T} \quad$ Transverse Energy

$E_{\mathrm{T}} \quad$ "missing" Transverse Energy

HERWIG HERWIG MC simulation

GEANT GEANT MC simulation

$p_{T} \quad$ Transverse Momentum

MC@NLO Monte Carlo simulation at Next to Leading Order

PYTHIA PYTHIA MC simulation 


\section{Foreword}

The work presented in this thesis is done in conjunction with the entire DØ collaboration. I did not build the D $\varnothing$ detector, nor did I create the reconstruction algorithms necessary for analyzing the data. I did take a series of detector data acquisition (DAQ) shifts on the DØ detector during the summer of 2010, although the data collected during that time period are not used in the analysis presented here. I did not make the simulated data samples used to model signal and background processes, but used those provided by the D $\varnothing$ top group. I did change the formatting of these samples so that we could use them for acceptance studies.

The majority of my contributions lie in Chapter 7, the analysis section. The skeletal structure of the analysis is based on code from the original asymmetry measurement (see Reference [1]) given to me by Amnon Harel. I made many changes to the analysis, including adjusting it for the format of the $\mathrm{D} \varnothing$ top group and expanding on it. The technique for regularized unfolding was developed by Amnon Harel, but I carried out the changes necessary to link this technique to data and simulation, and to check for systematic effects. 


\section{Introduction}

This thesis presents work which has recently been published in Physical Review $\mathrm{D}$ [2]. The goal of this thesis is to explain the measurement of the forwardbackward asymmetry in $t \bar{t}$ production and the results of the measurement. Specifically, this document aims to answer the following questions:

i What is a forward-backward asymmetry?

ii What are $t \bar{t}$ events?

iii How does one measure the forward-backward asymmetry?

iv Why is this asymmetry interesting?

\subsection{Quick and hole-filled history of particle physics}

Throughout the twentieth century and up to the present, researchers have been working to find and understand the interactions of the smallest portions of matter through what is now refered to as the discipline of high energy physics. Since Rutherford's [3] idea to bombard gold foil with energetic particles to understand the structure of atoms, physicists have been extending this technique, revealing a complex picture of the subatomic world.

As a result of this search, many conservation laws once held sacrosanct have been toppled, leading to a world where right differs from left, and (luckily for our existence) matter behaves differently than antimatter. The first violation of an assumed conservation law was predicted in 1956 by Lee and Yang [4]. An 
experiment assembled by $\mathrm{Wu}$ and collaborators [5] found this violation to exist in nature in 1957, which was quickly and independently verified by Lederman and collaborators [6]. Since then a thorough investigation of physics at subatomic scales has been underway, which is currently manifested in the construction of massive particle colliders, colloquially known as atom smashers.

The culmination of all of the experiments conducted at colliders through the years is a mathematical framework referred to as the standard model of particle physics (SM).

\subsection{The standard model}

The standard model of particle physics is analogous to the periodic table of elements, where the chemical elements are organized by their atomic structure. In the SM, particles are arranged into two groups: matter and force carriers. The particles making up matter are the building blocks for all of the visible universe. There two types of matter particles: quarks and leptons. At the temperatures that life as we know it exists, up and down quarks combine to form atomic nuclei. The most familiar example of a lepton is the electron, of which humanity's understanding is so great that an entire field of technology has arisen based on it: electronics. For the force carriers, the photon, the carrier of electromagnetism, is the particle people experience on a daily basis. Our understanding of the photon has led to another branch of technology: optics.

Along with the electromagnetic force, which is carried, or mediated, by the photon of quantum electrodynamics (QED), three other known forces exist within nature: the weak interaction, mediated by the $W^{+}, W^{-}$and $Z$ bosons; the strong interaction, mediated by eight different gluons and described by quantum chromodynamics (QCD); and gravity, which is hypothesized to be mediated by the graviton. The first three sets of particles have been observed and are an integral part of the standard model. The force carriers are referred to as bosons because of the integer size of their internal bar magnets, or spins. In contrast to the force carriers, particles making up matter are referred to as fermions because of the half-integer nature of their spins.

There are three sets of fermions, called families, which each contain two quarks, 


\begin{tabular}{|l|c|c|c|c||c|}
\hline \multicolumn{5}{|c|}{ Matter } & Force Carriers \\
\hline & Electric Charge & Family 1 & Family 2 & Family 3 & \\
\hline \multirow{3}{*}{ quarks } & $+2 / 3$ & $u$ & $c$ & $t$ & $\gamma$ \\
& $-1 / 3$ & $d$ & $s$ & $b$ & $W^{-}, W^{+}$ \\
leptons & -1 & $e$ & $\mu$ & $\tau$ & $Z$ \\
& 0 & $\nu_{e}$ & $\nu_{\mu}$ & $\nu_{\tau}$ & $g$ \\
antiquarks & $-2 / 3$ & $\bar{u}$ & $\bar{c}$ & $\bar{t}$ & \\
& $+1 / 3$ & $\bar{d}$ & $\bar{s}$ & $\bar{b}$ & \\
& +1 & $\bar{e}$ & $\bar{\mu}$ & $\bar{\tau}$ & \\
& 0 & $\bar{\nu}_{e}$ & $\bar{\nu}_{\mu}$ & $\bar{\nu}_{\tau}$ & \\
\hline
\end{tabular}

Table 2.1 Table of particles in the standard model.

a lepton and a neutrino. In total there are six flavors of quarks, three neutrinos and three leptons. All of the particles carry a weak charge and interact with the weak force carriers $W$ and $Z$. All of the particles, except for neutrinos, are electrically charged and interact with the electromagnetic force, mediated by $\gamma$. Only the quarks have a strong, or color, charge and participate in the strong interactions transmitted by gluons, $g$. In addition, quarks are fractionally electrically charged, with up-type quarks (top row of Table 2.1) having a charge of $+\frac{2}{3}$ and down-type quarks (second row of Table 2.1) having a charge of $-\frac{1}{3}$. If the idea of fractional electric charges seems strange, just imagine that $\frac{1}{3}$ is the normal unit of electric charge and the charge of the electron is -3 . To double the fun, each type of particle also has an antimatter twin. Table 2.1 shows all of the particles in the standard model.

\subsection{Perturbative QCD}

One of the standard ways to make predictions from a model is to use perturbation theory. Given a coupling strength, such as the coupling strength of quantum electrodynamics, $\alpha$, the theory can be expanded to different orders with terms containing $\alpha^{2}$ (leading order), $\alpha^{3}$ (next-to-leading order), $\alpha^{4}$ (next-to-next-toleading order) and so on. For calculations with $\alpha$, which is $\approx \frac{1}{137}$ at low energies, the first term offers enough precision. For instance, at order $\alpha^{2}$, QED can be used to calculate the cross section $(\sigma)$ for $e^{+} e^{-} \rightarrow \gamma \rightarrow \mu^{+} \mu^{-}$. The cross section is 
likelihood for a particular interaction to take place.

For QCD, the coupling strength, $\alpha_{s}$, is a dependent on the energy, or distance, of the interaction taking place. Because $\alpha_{s}$ is not constant with energy, this behavior is known as a "running" coupling. In QED, $\alpha$ also changes at shorter and shorter distance scales, but at a much slower pace. The running coupling strength leads to a property of QCD known as asymptotic freedom [7, 8]. At extremely high energies, $\alpha_{s}$ approaches 0 , and the color charge essentially goes to zero, meaning that quarks and gluons stop interacting with each other. On the flip side, at low energies and large distance scales, $\alpha_{s}$ grows so large that color confinement occurs and quarks exist as bound states, glued together in colorless configurations. These properties can be seen in Equation 2.1 [9]:

$$
\alpha_{s}\left(Q^{2}\right)=\frac{12 \pi}{\left(33-2 n_{f}\right) \log \left(\frac{Q^{2}}{\Lambda^{2}}\right)}
$$

where $Q^{2}$ is the momentum transfer, $n_{f}$ is the number of quark flavors and $\Lambda$ is the QCD scale parameter, about $210 \mathrm{MeV}$ [10]. At energies of $Q^{2}=M_{Z}^{2}=$ $(90 \mathrm{GeV})^{2}$, close to the energy scale for top quark pair production, $\alpha_{s} \approx \frac{1}{10}[11]$ and $\alpha \approx \frac{1}{129}$ [12]. Comparing these two numbers, it is obvious that QCD plays a much larger role at hadron colliders than QED. Because of the high momentum transfers involved, perturbative QCD (pQCD) can be put to use to make calculations for the Tevatron. Another method, called Lattice QCD [13], is a non-perturbative approach used to predict the masses of bound states of quarks, such as the neutron [11], as well as $\alpha_{s}\left(M_{Z}\right)$ and $\Lambda$.

\subsection{The top quark}

Discovered in 1995 at the Tevatron ${ }^{1}$ by the CDF [14] and D $\varnothing$ [15] collaborations, the top quark is the heaviest elementary particle currently known to science. At a mass of $172.9 \pm 0.6$ (stat) \pm 0.9 (syst) $\mathrm{GeV}$ [16], the top quark is about 190 times heavier than the proton, which weighs $0.981 \mathrm{GeV}$. The top quark has an extremely short lifetime of $5 \times 10^{-25} \mathrm{~s}$. Because the lifetime of the top quark is

\footnotetext{
${ }^{1}$ Thus it is not a coincidence that this current measurement also takes place at the Tevatron.
} 
so short, QCD does not have time to take effect and the top quark is the only "bare" quark observed.

The top quark is about as massive as a gold atom. This equivalence can be shown using the conversion formulae, $173 \mathrm{GeV} \times 1.78 \times 10^{-24} \frac{\mathrm{g}}{\mathrm{GeV}}=3.1 \times 10^{-22} \mathrm{~g}$ and $196.97 \frac{\mathrm{g}}{\mathrm{mol}} /\left(6.02 \times 10^{23} \frac{\mathrm{atoms}}{\mathrm{mol}}\right)=3.3 \times 10^{-22} \mathrm{~g} .{ }^{2}$ On the other hand, top quarks are far more expensive than gold. At current prices, gold costs $\$ 55$ per gram. Estimating of the price of an individual top quark in a collider is a bit harder. Combining an estimate of the cost of the construction and operation of the Tevatron and the $\mathrm{D} \varnothing$ detector, about $\$ 1$ billion to $\$ 4$ billion, and an estimate of the number of top quarks produced, $5.4 \mathrm{fb}^{-1} \times 7,000 \mathrm{fb} \approx 35,000$ top quarks, leads to a ball park cost of $\$ 25,000$ to $\$ 100,000$ per top quark. Top quarks are worth far more than their weight in gold.

\subsection{Top quark production at the Tevatron}

At the Tevatron, where protons, made up of quarks, and antiprotons, made up of antiquarks, collide, the dominant process for creating top quarks is called top pair production. At leading order in QCD $\left(\alpha_{s}^{2}\right)$, either a pair of gluons or a quarkantiquark pair collide to form a gluon, which decays to a $t \bar{t}$ pair. At the Tevatron, the main production mode at $85 \%$ is $q \bar{q} \rightarrow t \bar{t}$, as seen in Figure 2.1, while $g g \rightarrow t \bar{t}$ makes up only $15 \%$ of production. This ratio is important for this measurement, as will be explained later on. At the LHC, where both colliding beams are made up of protons, the ratio between $q \bar{q}$ and $g g$ is reversed. The cross section for $t \bar{t}$ production at the Tevatron $\left(\sigma_{t \bar{t}}\right)$ is theorized to be $\sim 7 \mathrm{pb}[17,18,19,20,21,22,23]$ and is measured to be $\sigma_{t \bar{t}}=7.78_{-0.64}^{+0.77} \mathrm{pb}[24]$ for the data sample being studied here.

Another mode of creation for top quarks at the Tevatron is single top production, where only one top quark (or antiquark) is made during a collision. The cross section for this process is smaller than for $t \bar{t}$ production, with a prediction of $\sigma_{t}=2.54 \pm 0.13 \mathrm{pb}$ (Figure 2.2) [25]. The latest combined single top production

\footnotetext{
${ }^{2}$ All conversion numbers are taken from wikipedia.org.
} 

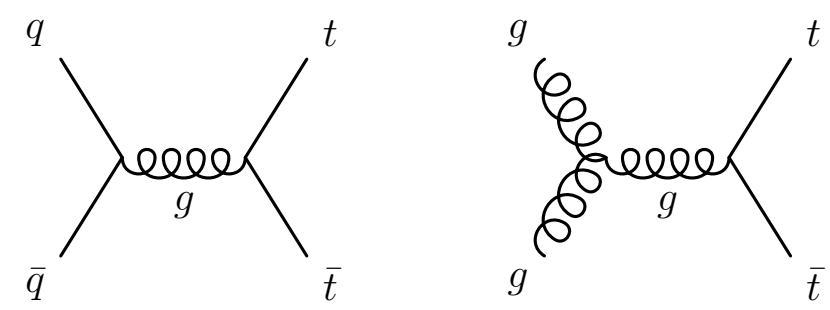

Figure 2.1 Feynman diagrams for $t \bar{t}$ production at the Tevatron, with $q \bar{q} \rightarrow t \bar{t}$ shown on the left and $g g \rightarrow t \bar{t}$ shown on the right.

cross section measurement at $\mathrm{D} \varnothing$ of $\sigma\left(p \bar{p} \rightarrow t b+t b q+X^{3}\right)=3.43_{-0.74}^{+0.73} \mathrm{pb}$ is in good agreement with the prediction [26].
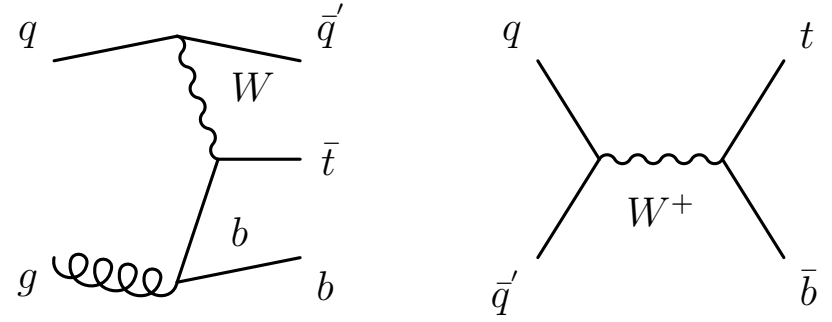

Figure 2.2 Feynman diagrams of single top production at the Tevatron, with $q g \rightarrow \bar{q}^{\prime} b \bar{t}$ ( $t$ channel, left) and $q \bar{q}^{\prime} \rightarrow t \bar{b}$ ( $s$ channel, right).

\subsection{Top quark decay}

The top quark is predicted to decay to a $W$ boson and a $b$ quark $(t \rightarrow W b)$ almost $100 \%$ of the time [16]. Recent DØ measurements have shown this preference may not hold exactly [27]. For the purposes of this measurement, the probability of $t \rightarrow W b$ is considered to be exactly one. The daughters from the $W$ boson decay determine the type of decay for the top quark. The $W$ boson has two different types of decays. For the first type, called the "leptonic" channel, the $W$ boson decays to a lepton and a neutrino, $W \rightarrow l_{i} \nu_{i}$, where $i$ is either the electron flavor, the muon flavor or the tau flavor of lepton-neutrino pair. In the second type of decay, called the "hadronic" channel, the $W$ boson decays to two quarks, either an up and a down quark $(W \rightarrow u d)$ or a charm and strange quark $(W \rightarrow c s)$. In the hadronic case, the daughter quarks can have one of three colors. Because all

\footnotetext{
${ }^{3}$ In the equation the $X$ is for anything else that might be included in production, such as an additional gluon or a photon.
} 
of the potential daughter products are small in mass compared to the $W$ boson, kinematic or "phase space" requirements do not play a large role. Doing a "back of the envelope" calculation and treating all of the daughter products equally shows that the $W$ boson has a $\frac{1}{3}$ chance of decaying to leptons and neutrinos, and a $\frac{2}{3}$ probability of decaying to quarks. Looking at the literature shows that this approximation is good [16].

In $t \bar{t}$ decay, there are two $W$ bosons and two $b$ quarks, $t \bar{t} \rightarrow W^{+} b W^{-} \bar{b}$. There are three different ways the pair of $W$ bosons can decay. Both $W$ bosons can decay to leptons and neutrinos, $t \bar{t} \rightarrow b l \nu \bar{b} \bar{l} \nu$, the "di-lepton" decay mode. Both $W$ bosons can decay to quarks, the "fully hadronic" or "all jets" mode. Or the $W$ bosons can decay to different types of quarks and leptons, the "semi-leptonic" or "lepton+jets" mode. For the current measurement, we use the lepton+jets $(l+$ jets $)$ mode of decay. Tau leptons are much harder to detect than electrons or muons. To keep things simple, only electrons or muons are used in this measurement, although a small fraction of events, about $6 \%$, are electrons or muons resulting from the decay of an intermediate tau lepton. Doing the math for the decay of two $W$ bosons, one with a probability to decay to an electron or muon and a neutrino and the other with a probability to decay to quarks, $2\left(\frac{2}{9}\right)\left(\frac{6}{9}\right)=\frac{24}{81}$, or approximately $30 \%$ of $t \bar{t}$ decays occur via the $l+$ jets mode.

Figure 2.3 shows an example of Feynman diagrams for the $e+$ jets and the $\mu+$ jets decay channels.

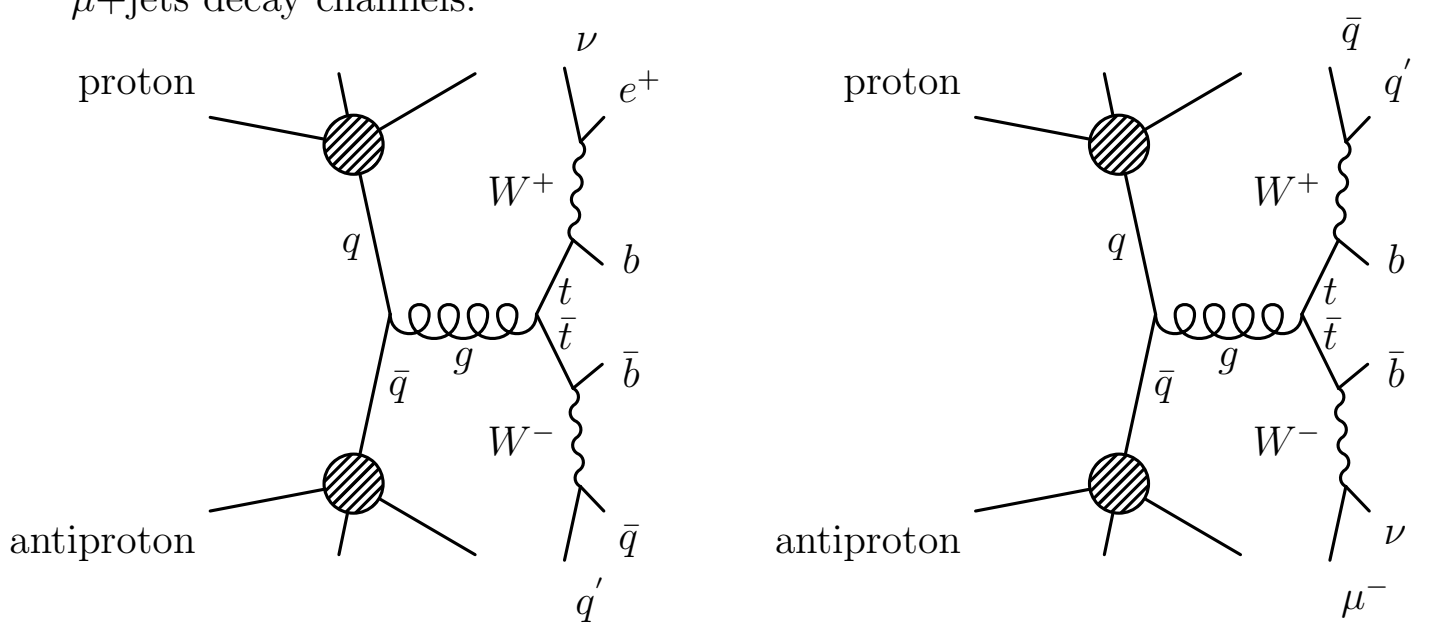

Figure 2.3 Example Feynman diagrams of the $e+$ jets channel (left) and the $\mu+$ jets channel (right) for $t \bar{t}$ decay. 


\subsection{Backgrounds to top quark decay}

In particle physics, processes that mimic the detector signature of the main physical process being studied are called backgrounds. One measure of the purity of a sample is the signal-to-background ratio. This is analogous to the signal-to-noise ratio. In the case of radio broadcasts, when the signal-to-noise ratio is low, one hears static interfering with a song. There are multiple background processes to top quark decay. Some processes have the same daughter particles as $t \bar{t}$ decay. Other processes have different daughter particles but are still detected as a top quark decays due to the imperfect nature of the $\mathrm{D} \emptyset$ detector.

There are multiple backgrounds that decay to the lepton+jets channel with the same daughter particles as $t \bar{t}$ decay: a $W$ boson produced in association with quarks and gluons ( $W+$ jets), single top production, $W W, Z Z$ and $W Z$ production and the production of a $Z$ boson in association with quarks and gluons $(Z+$ jets). The largest background, in terms of events produced, which passes the $t \bar{t}$ selection criteria, is $W+$ jets.

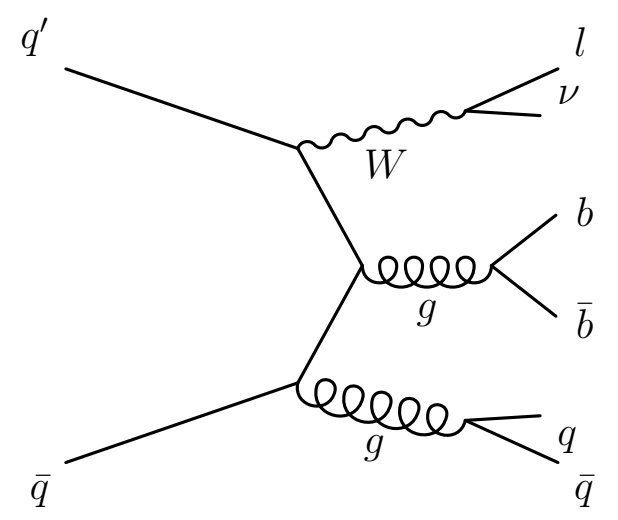

Figure 2.4 Example Feynman diagram of $W+$ jets background for $t \bar{t}$ decay.

Another set of background processes consists of the production of quarks and gluons that mimic $t \bar{t}$ decay in the detector. Because these processes originate only from quarks and gluons, which are detected as jets, the collection of these processes is known as the multijet background. 


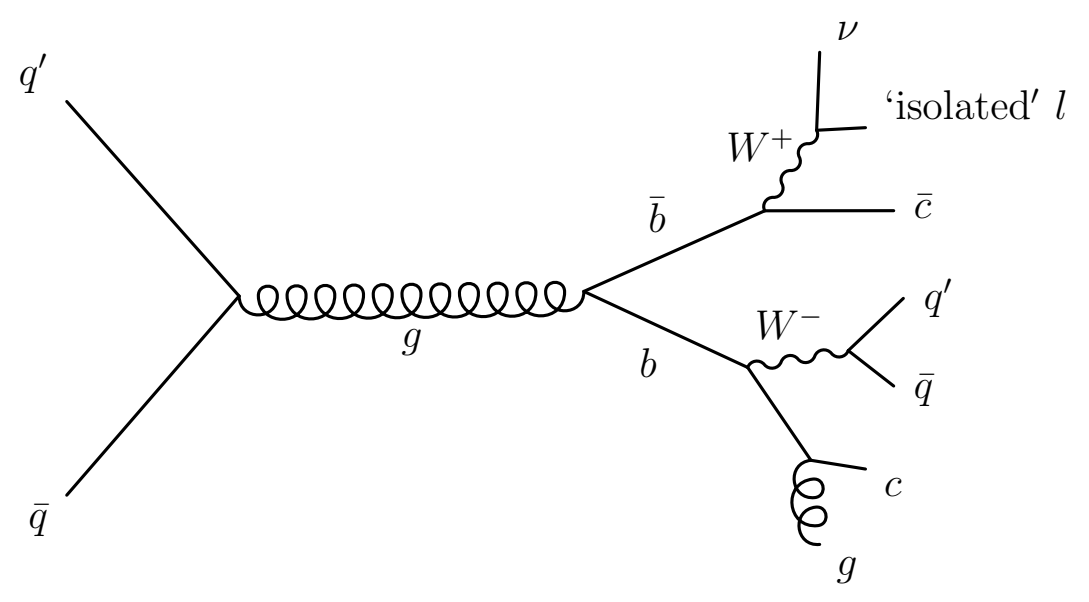

Figure 2.5 Example Feynman diagram of MJ background for $t \bar{t}$ decay.

\subsection{Observable quantities}

To make a measurement in physics, one needs something to measure. This may sound tautological, but many important discoveries have been made by measuring the right thing. The things that one can measure in an experiment are known as observables. In this analysis, we measure two different observables, $\Delta y$ and $q_{l} y_{l}$. The first observable, $\Delta y$, is defined as

$$
\Delta y=y_{t}-y_{\bar{t}},
$$

where $y$ is the rapidity, defined as

$$
y=\frac{1}{2} \log \left(\frac{E+p_{z}}{E-p_{z}}\right),
$$

where $E$ is the energy of a particle, $p_{z}$ is the component along the $z$ direction and the $z$ direction is parallel to the beam axis [1]. In the ultra-relativistic approximation, that is when the energy of a particle is equal to the magnitude of the momentum of a particle, the rapidity reduces to the pseudorapidity, $\eta$, 


$$
\begin{aligned}
y & =\frac{1}{2} \log \left(\frac{E+E \cos (\theta)}{E-E \cos (\theta)}\right)=\frac{1}{2} \log \left(\frac{1+\cos (\theta)}{1-\cos (\theta)}\right) \\
& =\frac{1}{2} \log \left(\frac{\frac{1}{2}\left(1+\cos \left(2 \frac{\theta}{2}\right)\right)}{\frac{1}{2}\left(1-\cos \left(2 \frac{\theta}{2}\right)\right)}\right)=\frac{1}{2} \log \left(\frac{\cos \left(\frac{\theta}{2}\right)^{2}}{\sin \left(\frac{\theta}{2}\right)^{2}}\right) \\
& =\frac{1}{2} \log \left(\tan \left(\frac{\theta}{2}\right)^{-2}\right)=-\log \left(\tan \left(\frac{\theta}{2}\right)\right)=\eta,
\end{aligned}
$$

where $\theta$ is the angle with respect to the beam axis. The angle $\theta=0$ corresponds to the proton direction, while $\theta=\pi$ corresponds to the antiproton direction. Although $\Delta y$ looks complicated and depends on both the energies and the momenta parallel to the beam line of the top quarks, this quantity is a function of the angle of the top quarks to the beam line in the $t \bar{t}$ center of momentum frame. In the $t \bar{t}$ center of momentum frame, if the total energy is $E$, the energy of each top quark is $\frac{E}{2}$, due to conservation of energy. Each top quark also has equal and opposite momentum, $\vec{p}_{t}=-\vec{p}_{\vec{t}}$, from conservation of momentum. Substituting these terms in to Equation 2.2 leads to

$$
\begin{aligned}
\Delta y & =\frac{1}{2} \log \left(\frac{E^{t}+p_{z}^{t}}{E^{t}-p_{z}^{t}}\right)-\frac{1}{2} \log \left(\frac{E^{\bar{t}}+p_{z}^{\bar{t}}}{E^{\bar{t}}-p_{z}^{\bar{t}}}\right)=\frac{1}{2} \log \left(\left(\frac{\frac{E}{2}+p \cos \left(\theta_{t \bar{t}}\right)}{\frac{E}{2}-p \cos \left(\theta_{t \bar{t}}\right)}\right)^{2}\right) \\
& =\log \left(\frac{1+\beta_{t} \cos \left(\theta_{t \bar{t}}\right)}{1-\beta_{t} \cos \left(\theta_{t \bar{t}}\right)}\right)=2 \operatorname{atanh}\left(\beta_{t} \cos \left(\theta_{t \bar{t}}\right)\right),
\end{aligned}
$$

where $\cos \left(\theta_{t \bar{t}}\right)$ is the cosine of the angle between the top quark and the beam line and $\beta_{t}=\sqrt{1-\frac{4 m_{t}^{2}}{E}}=\sqrt{1-\frac{4 m_{t}^{2}}{\hat{S}}}$ is the relativistic top velocity. A nice feature of $\Delta y$ is invariance to boosts along the direction of the beam line. Using the Lorentz transformations, $E \rightarrow \gamma E-\beta \gamma p_{z}$ and $p_{z} \rightarrow \gamma p_{z}-\beta \gamma E$, leads to: 


$$
\begin{aligned}
y^{\prime} & =\frac{1}{2} \log \left(\frac{E^{\prime}+p_{z}^{\prime}}{E^{\prime}-p_{z}^{\prime}}\right)=\frac{1}{2} \log \left(\frac{\gamma E-\beta \gamma p_{z}+\gamma p_{z}-\beta \gamma E}{\gamma E-\beta \gamma p_{z}-\gamma p_{z}+\beta \gamma E}\right) \\
& =\frac{1}{2} \log \left(\frac{\gamma(1-\beta)}{\gamma(1+\beta)} \frac{E+p_{z}}{E-p_{z}}\right)=\frac{1}{2} \log \left(\frac{E+p_{z}}{E-p_{z}}\right)+\frac{1}{2} \log \left(\frac{1-\beta}{1+\beta}\right) \\
& =y+\frac{1}{2} \log \left(\frac{1-\beta}{1+\beta}\right)
\end{aligned}
$$

So $\Delta y^{\prime}=y_{t}^{\prime}-y_{\bar{t}}^{\prime}=y_{t}+\frac{1}{2} \log \left(\frac{1-\beta}{1+\beta}\right)-y_{\bar{t}}-\frac{1}{2} \log \left(\frac{1-\beta}{1+\beta}\right)=\Delta y$ and $\Delta y$ is invariant to boosts along the beam line. If a boost occurs perpendicular to the beam line, this invariance no longer holds.

To measure $\Delta y$, the rapidities of both the hadronically decaying top quark $\left(y_{t, \text { had }}\right)$ and the leptonically decaying top quark $\left(y_{t, \text { lep }}\right)$ are reconstructed. The charge of the lepton, $q_{l}$, decides which reconstructed top is the quark and which is the antiquark, leading to:

$$
\Delta y=q_{l}\left(y_{t, \text { lep }}-y_{t, \text { had }}\right)
$$

The second observable, the charge-signed lepton rapidity or $q_{l} y_{l}$, the product of the lepton charge and the lepton rapidity $\left(y_{l}\right)$, is much simpler than $\Delta y$. Since leptons are much smaller in mass than the top quark and the $W$ boson, they are produced with relativistic velocities, leading to $y_{l} \approx \eta_{l}$. The relative brevity of the description of $q_{l} y_{l}$ compared the description of $\Delta y$ should not detract from the importance of this variable. As a simple quantity that is easily measured by the detector, much can be learned from $q_{l} y_{l}$ without complicated reconstruction techniques. Not just a simple crosscheck to $\Delta y, q_{l} y_{l}$ is a strong observable in its own right.

\subsection{Definition of forward-backward asymmetry}

As seen in Section 2.5, the measured $t \bar{t}$ production cross section is in good agreement with SM predictions. Therefore other measurements are necessary to probe for new physics and test the predictive power of the SM. One such measurement, the one described in this document, determines the difference between the ratios 


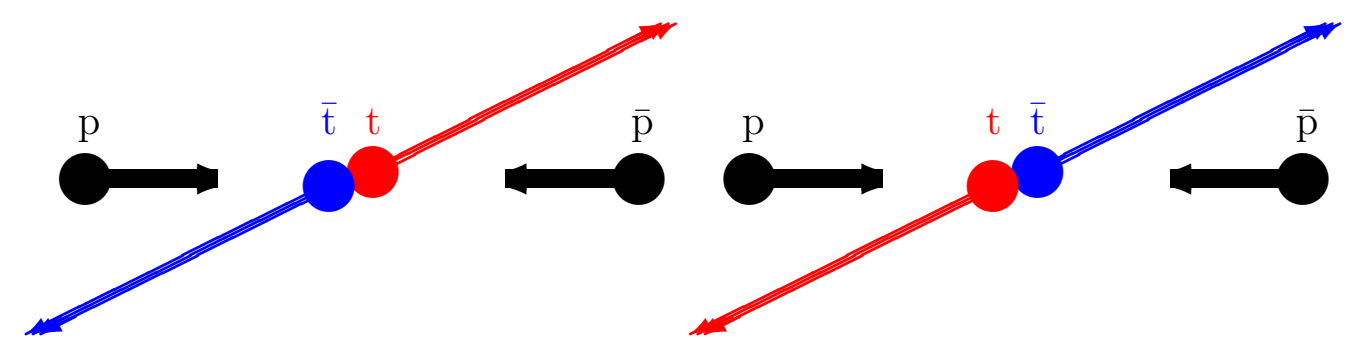

Figure 2.6 Examples of forward (left) and backward (right) events.

of cross sections, also known as the forward-backward asymmetry, $A_{\mathrm{FB}}$. There are many equivalent ways to define $A_{\mathrm{FB}}$. First, the total number of events can be defined such that:

$$
N=N_{F}+N_{B}
$$

where $N_{F}$ is the number of forward events, which means events that have $\Delta y>0$ or $q_{l} y_{l}>0$, and $N_{B}$ is the number of backward events, where $\Delta y<0$ or $q_{l} y_{l}<0$. For an illustration of the difference between forward and backward events, see Figure 2.6. The ratio of the number of forward to the number of backward events is:

$$
R=\frac{N_{F}}{N_{B}}
$$

The forward-backward asymmetry, $A_{\mathrm{FB}}$, is defined as:

$$
A_{\mathrm{FB}}=\frac{N_{F}-N_{B}}{N_{F}+N_{B}}=\frac{N_{F}}{N}-\frac{N_{B}}{N}=f_{F}-f_{B}=\frac{\frac{N_{F}}{N_{B}}-1}{\frac{N_{F}}{N_{B}}+1}=\frac{R-1}{R+1}
$$

The uncertainty on $A_{\mathrm{FB}}$ is founding using:

$$
(\Delta F(x, y))^{2}=\left(\frac{d F}{d x}\right)^{2}(\delta x)^{2}+\left(\frac{d F}{d y}\right)^{2}(\delta y)^{2},
$$

where $F$ is some function, in this case $A_{f b}$, and $x$ and $y$ are variables that the function depends on, $N_{F}$ and $N_{B}$. 


$$
\begin{gathered}
\frac{d A_{f b}}{d N_{F}}=\frac{1}{N}-\left(\frac{N_{F}-N_{B}}{N^{2}}\right)=\frac{2 N_{B}}{N^{2}} \\
\frac{d A_{f b}}{d N_{B}}=\frac{-1}{N}-\left(\frac{N_{F}-N_{B}}{N^{2}}\right)=\frac{-2 N_{F}}{N^{2}}
\end{gathered}
$$

These steps lead to the uncertainty for $A_{\mathrm{FB}}$ :

$$
\left(\Delta A_{f b}\right)^{2}=\left(\frac{2 N_{B}}{N^{2}}\right)^{2}\left(\delta N_{F}\right)^{2}+\left(\frac{-2 N_{F}}{N^{2}}\right)^{2}\left(\delta N_{B}\right)^{2} .
$$

For data, the Poisson uncertainty (Equation 2.15) is used for the uncertainty on the number of events, leading to Equation 2.16.

$$
\begin{gathered}
\delta N=\sqrt{N} \\
\Delta A_{f b}=\frac{2}{N}\left[\frac{N_{F} N_{B}}{N}\right]^{\frac{1}{2}}
\end{gathered}
$$

Putting things together, the forward-backward asymmetry of the $\Delta y$ distribution is

$$
A_{\mathrm{FB}}=\frac{N(\Delta y>0)-N(\Delta y<0)}{N(\Delta y>0)+N(\Delta y<0)},
$$

and the forward-backward asymmetry of the $q_{l} y_{l}$ distribution is

$$
A_{\mathrm{FB}}^{l}=\frac{N\left(q_{l} y_{l}>0\right)-N\left(q_{l} y_{l}<0\right)}{N\left(q_{l} y_{l}>0\right)+N\left(q_{l} y_{l}<0\right)} .
$$

\subsection{Forward-backward asymmetry in $t \bar{t}$ produc- tion}

The first paper outlining a procedure for observing an asymmetry in $t \bar{t}$ production at the Tevatron appeared in 1998 [28, 29]. Predictions that an asymmetry might be present in $t \bar{t}$ production came as early as the late 1980's, before the top quark was even discovered [30,31]. Further studies have added to more details to the prediction. 
The first measurements of the $t \bar{t}$ asymmetry were published in 2008 by the DØ [1] and CDF [32] collaborations. This thesis describes the update and expansion of the first $\mathrm{D} \emptyset$ measurement. The CDF collaboration also recently updated and expanded their first measurement with $5.3 \mathrm{fb}^{-1}$ [33] and presented an $A_{\mathrm{FB}}$ measurement in the dilepton channel [34].

At leading order (LO) in quantum chromodynamics (QCD), no asymmetry is present. The gluon contents of the proton and antiproton are equal, so no asymmetry arises from gluon-gluon fusion at any order. When an incoming $q \bar{q}$ pair annihilates into a gluon, all memory of the differences in direction between the quark and antiquark is erased, which means there is a lack of asymmetry. The leading order calculation for QCD is essentially the same as the calculation of $e^{+} e^{-} \rightarrow \gamma \rightarrow \mu^{+} \mu^{-}$at leading order in QED, as shown in Reference [9]. This calculation leads to a differential cross section that is proportional to $1+\cos (\theta)^{2}$, where $\theta$ is the angle of between the outgoing $\mu^{+}$and the incoming $e^{+}$. There is no asymmetric term in the differential cross section and no forward-backward asymmetry appears. Although the calculation for $q \bar{q} \rightarrow t \bar{t}$ at leading order is slightly more complicated due to the large mass of the top quark and the fact that eight gluons, rather than one photon, are the mediating particles, no asymmetric term arises.

When calculations are made at next-to-leading order (NLO) in QCD, a positive asymmetry appears. Reference [28] predicts an asymmetry of about $5 \%$ in the difference in direction between the $t$ and $\bar{t}$ quarks, driven by the interference of the diagrams in Figure 2.7. Reference [35] investigates the measurement of the asymmetry from an experimental perspective and was the first to suggest asymmetries based on $\eta_{l}$, stating, "First, experimentally the most reliable avenue for measuring the asymmetry is likely via the charged leptons, where both the direction and the sign of the charge can be determined with confidence." Due to limitations of the theoretical tools at the time of writing, Reference [35] does not make any predictions for $A_{\mathrm{FB}}^{l}$.

In addition, Reference [28] calculated the differential asymmetry for soft (low energy) and hard (high energy) gluon radiation, and found that the asymmetry is positive in regions of phase space with soft gluon radiation and negative in regions of phase space with hard gluon radiation. In the more technical words of Reference [35], "It was found [in Ref. [28]] that the virtual contribution pro- 

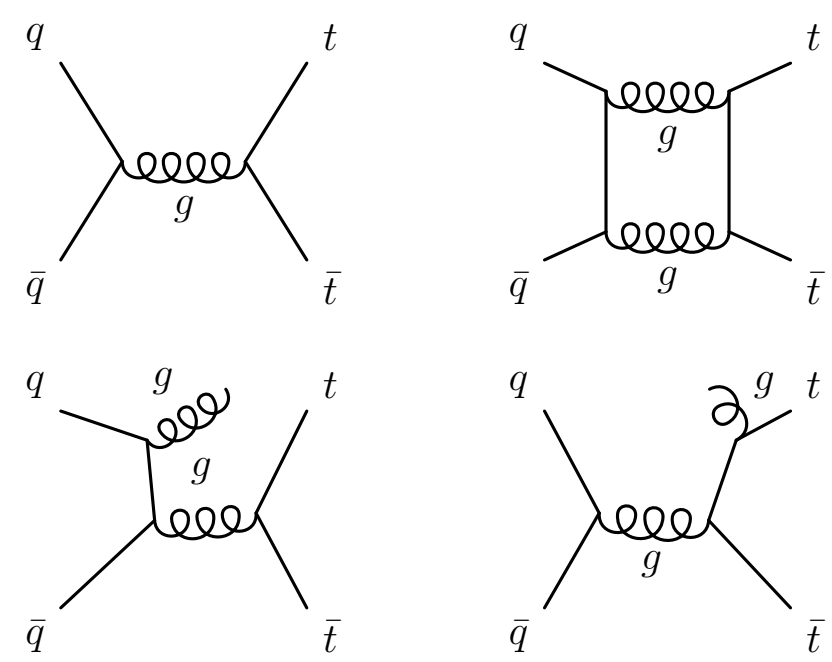

Figure 2.7 Example Feynman diagrams that interfere to produce $A_{\mathrm{FB}}$ in $t \bar{t}$ production.

duces an asymmetry opposite in sign to and larger than that of the real emission component." This dependence leads to a rule of thumb for the experimental measurement: in events with extra gluon radiation, such as an additional jet, the asymmetry is going to be smaller than in events without additional gluon radiation.

The first prediction of the asymmetry for events containing one or more additional jets, $q \bar{q} \rightarrow t \bar{t}+$ jet, was carried out in Reference [36]. They found an asymmetry of $\sim-2 \%$ for NLO in QCD, compared to an asymmetry of $\sim-7 \%$ for LO in QCD. Reference [37] independently verified the $t \bar{t}+$ jet calculation for $A_{\mathrm{FB}}$ and argues that the large change in the asymmetry from LO to NLO is "natural" and further calculation at higher orders in QCD should be more stable.

A more recent calculation at NLO in QCD with higher-order corrections for soft gluon radiation (NNLL), carried out in Reference [38] predicts an asymmetry of about $7 \%$ for inclusive $t \bar{t}$ production (with or without an extra jet). Reference [39] includes contributions from electroweak interactions in the asymmetry calculation and finds that the inclusion of the photon has a large enhancement, while effects from the $Z$ boson are negligible. The resulting asymmetry from QCD combined with QED is about $9 \%$. An additional contribution to the asymmetry from $b \bar{b} \rightarrow t \bar{t}$ has not been included in the predictions yet [40].

There are two different ways that the asymmetry is calculated. When only 
including contributions from QCD, the perturbative expansion in the asymmetry is:

$$
A_{\mathrm{FB}}=\frac{\left(\alpha_{s}^{2} \sigma_{\mathrm{LO}}^{\mathrm{F}}+\alpha_{s}^{3} \delta\left(\sigma_{\mathrm{NLO}}^{\mathrm{F}}\right)+\alpha_{s}^{4} \delta\left(\sigma_{\mathrm{NNLO}}^{\mathrm{F}}\right)+\ldots\right)-\left(\alpha_{s}^{2} \sigma_{\mathrm{LO}}^{\mathrm{B}}+\alpha_{s}^{3} \delta\left(\sigma_{\mathrm{NLO}}^{\mathrm{B}}\right)+\alpha_{s}^{4} \delta\left(\sigma_{\mathrm{NNLO}}^{\mathrm{B}}\right)+\ldots\right)}{\left(\alpha_{s}^{2} \sigma_{\mathrm{LO}}^{\mathrm{F}}+\alpha_{s}^{3} \delta\left(\sigma_{\mathrm{NLO}}^{\mathrm{F}}\right)+\alpha_{s}^{4} \delta\left(\sigma_{\mathrm{NNLO}}^{\mathrm{F}}\right)+\ldots\right)+\left(\alpha_{s}^{2} \sigma_{\mathrm{LO}}^{\mathrm{B}}+\alpha_{s}^{3} \delta\left(\sigma_{\mathrm{NLO}}^{\mathrm{B}}\right)+\alpha_{s}^{4} \delta\left(\sigma_{\mathrm{NNLO}}^{\mathrm{B}}\right)+\ldots\right)}
$$

where $\sigma^{\mathrm{F}}$ is the contribution to the $t \bar{t}$ cross section from the section of phase space where $\Delta y>0$ and $\sigma^{\mathrm{B}}$ is the contribution from the section of phase space where $\Delta y<0$. The terms $\delta(\sigma)$ are the changes in the magnitudes for the cross sections from the previous order of calculation, e.g. $\alpha_{s} \delta\left(\sigma_{\mathrm{NLO}}\right)=\sigma_{\mathrm{NLO}}-\sigma_{\mathrm{LO}}$ for the difference between the leading order and next-to-leading-order calculations. These changes do not need to be positive. There are two ways to expand this series. The most straight forward way is to expand the numerator $\left(\Delta \sigma=\sigma^{\mathrm{F}}-\sigma^{\mathrm{B}}\right)$ and the denominator $(\sigma)$ separately to calculate $A_{\mathrm{FB}}=\frac{\Delta \sigma}{\sigma}$. To do this expansion, next-to-leading-order MC simulation programs, such as MC@NLO [41, 42] and MCFM [43], calculate the differential cross section $\frac{\partial \sigma}{\partial(\Delta y)}$. The differential cross section is integrated to obtain the forward and backward contributions:

$$
\sigma^{\mathrm{B}}=\int_{-\infty}^{0} \frac{\partial \sigma}{\partial(\Delta y)} d(\Delta y) \text { and } \sigma^{\mathrm{F}}=\int_{0}^{+\infty} \frac{\partial \sigma}{\partial(\Delta y)} d(\Delta y)
$$

As discussed in Reference [38], most theoretical calculations use another choice of perturbation, expanding $A_{\mathrm{FB}}$ in orders of $\alpha_{s}$ :

$$
A_{\mathrm{FB}}=\alpha_{s}\left(\frac{\Delta \sigma_{\mathrm{NLO}}+\alpha_{s}\left(\Delta \sigma_{\mathrm{NNLO}}\right)+\ldots}{\sigma_{\mathrm{LO}}+\alpha_{s} \delta\left(\sigma_{\mathrm{NLO}}\right)+\ldots}\right)
$$

As stated in Reference [17], NLO+NNLL calculations of the asymmetry are more stable for the two choices of perturbation than purely NLO calculations. Still, the fact remains that the number that results from the perturbative expansion of $A_{\mathrm{FB}}$ separately for the numerator and denominator is different than the number that results from the expansion of $A_{\mathrm{FB}}$ in orders of $\alpha_{s}$ by a factor of:

$$
\frac{\frac{\Delta \sigma_{\mathrm{NLO}}}{\sigma_{\mathrm{LO}}+\alpha_{s} \delta\left(\sigma_{\mathrm{NLO}}\right)}}{\frac{\Delta \sigma_{\mathrm{NLO}}}{\sigma_{\mathrm{LO}}}}=\frac{\sigma_{\mathrm{LO}}}{\sigma_{\mathrm{LO}}+\alpha_{s} \delta\left(\sigma_{\mathrm{NLO}}\right)}=\frac{1}{1+\frac{\alpha_{s} \delta\left(\sigma_{\mathrm{NLO}}\right)}{\sigma_{\mathrm{LO}}}}
$$

Theorists readily divulge of the existence of two different perturbation schema. 
Still, the use of the expansion in orders of $\alpha_{s}$ seems somewhat strange, and is only possible due to the cancellation of the forward and backward parts of the LO $t \bar{t}$ cross section. Taking some cross sections for a "worst case" scenario from Reference [17], this factor is approximately $\frac{1}{1+\frac{5.8-4.5}{4.5}}=78 \%$, meaning that there is up to a $25 \%$ difference between the two predictions.

The sole prediction for the lepton-based asymmetry is calculated in Reference [44]. They find that $A_{\mathrm{FB}}^{l}$ is about $3.3 \%$, but simulated selection criteria are included in this calculation. No calculation of $A_{\mathrm{FB}}^{l}$ at the production level without any selection criteria is currently published, but a currently unpublished value is approximately $4 \%$, taken from internal $\mathrm{D} \varnothing$ correspondence with the authors from Reference [44]. There is perhaps a slight bias in favor of $A_{\mathrm{FB}}$ over $A_{\mathrm{FB}}^{l}$, both experimentally and theoretically, but it appears the trend is headed in the other direction.

In summary, current predictions for $A_{\mathrm{FB}}$ range from $\sim 5 \%$ to $\sim 9 \%$, while current predictions for $A_{\mathrm{FB}}^{l}$ are $\sim 3 \%$ to $\sim 4 \%$. These predictions are the current state of the art, but may change in the future if and when additional orders of calculation are carried out at NNLO (order $\left.\alpha_{s}^{4}\right)$ in QCD.

\subsection{Asymmetry beyond the standard model}

Recently, due to somewhat large $A_{\mathrm{FB}}$ results from $\mathrm{D} \varnothing$ and CDF and a paucity of other top sector measurements that disagree with SM predictions, a number of models using new physics scenarios beyond the standard model (BSM) have been put forward. As the types of BSM models are both diverse and sundry, two general scenarios are considered here. Constructing new scenarios that are plausible (also known as model building) and increase the asymmetry is hard, as there are many constraints that already exist from a plethora of other collider measurements. For instance, if a proposed BSM scenario drastically increased the $t \bar{t}$ cross section at the Tevatron, this scenario would need to be discarded because of disagreement with the cross section measurements, which are in good agreement with SM predictions. New constraints arriving from the LHC are also limiting the space for new models to occupy.

A good summary of all the new types of models developed in light of the 
Tevatron $t \bar{t}$ asymmetry measurements can be found in Reference [45], along with a detailed list of further references. These models can be broken into two categories. The first type of model has a particle that is created by $q \bar{q}$ annihilation via the $s$ channel and then decays to $t \bar{t}$. These particles are known as massive color octets, a heavy version of the gluon. The second type of particle is exchanged between $q$ and $\bar{q}$, via the $t$ or the $u$ channel, converting the incoming $q$ to a $t$ or $\bar{t}$ and the incoming $\bar{q}$ to a $\bar{t}$ or a $t$. Some types of these interactions change the flavor of the quark but carry no electric charge of the their own, and are referred to as flavor-changing neutral currents (FCNC). Example Feynman diagrams from both types of models are shown in Figure 2.8. Other detector signatures which can either confirm or rule out these models include the production of same-sign top quarks in $p p$ collisions at the LHC, resonances in the $t \bar{t}$ invariant mass spectrum and dijet production.

Different models can also predict $A_{\mathrm{FB}}$ and $A_{\mathrm{FB}}^{l}$ values that are decoupled from each other, some of which have values of $A_{\mathrm{FB}}^{l}$ that are greater than $A_{\mathrm{FB}}$. Reference [46] investigates some of these scenarios, as well as ties in the asymmetry measurement with a set of other observables, such as the polarization of the $t$ quark, to constrain and search for BSM physics.

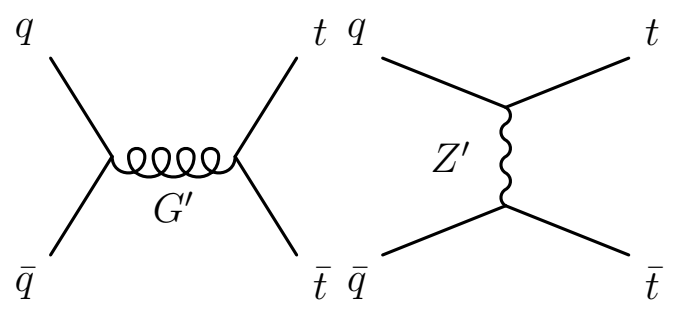

Figure 2.8 Feynman diagrams for $t \bar{t}$ production via massive color octets (left) and $t$-channel $Z^{\prime}$ exchange (right).

\subsection{Connecting theory to measurement}

One of the challenges of this measurement (and all measurements in high energy physics) is connecting the signatures in the detector with predictions from the standard model. At the most basic level there is the asymmetry actually produced in top quark pair events in nature. We can never know this perfectly. That is a 
fact of science. First of all, not all $t \bar{t}$ events are detected by $\mathrm{D} \varnothing$. The D $\varnothing$ detector does not have perfect " $4 \pi$ " coverage, that is D $\varnothing$ is not a complete sphere. Second, even when all of the daughter products from $t \bar{t}$ decays are within the volume of the $\mathrm{D} \varnothing$ detector, not all of the events are detected properly or pass the criteria necessary to differentiate them from background processes. These two effects make up a process that is called acceptance. The definition of acceptance is simple: acceptance is the process where events are accepted by the detector and recorded.

After the events are accepted by D $\varnothing$, they need to be reconstructed. Reconstruction means that the six decay products from the $t \bar{t}$ decay need to be identified correctly, answering the question "What particle did this energy, deposited in the detector, come from?" By far the best measured particles are the electrons and muons. The DØ detector has a harder time identifying and measuring the energies of the jets from the quark decays. Measuring the energy of the neutrino is the hardest for the detector, because neutrinos essentially never interact with the detector, leaving only an imbalance transverse energy.

Putting the sequence together:

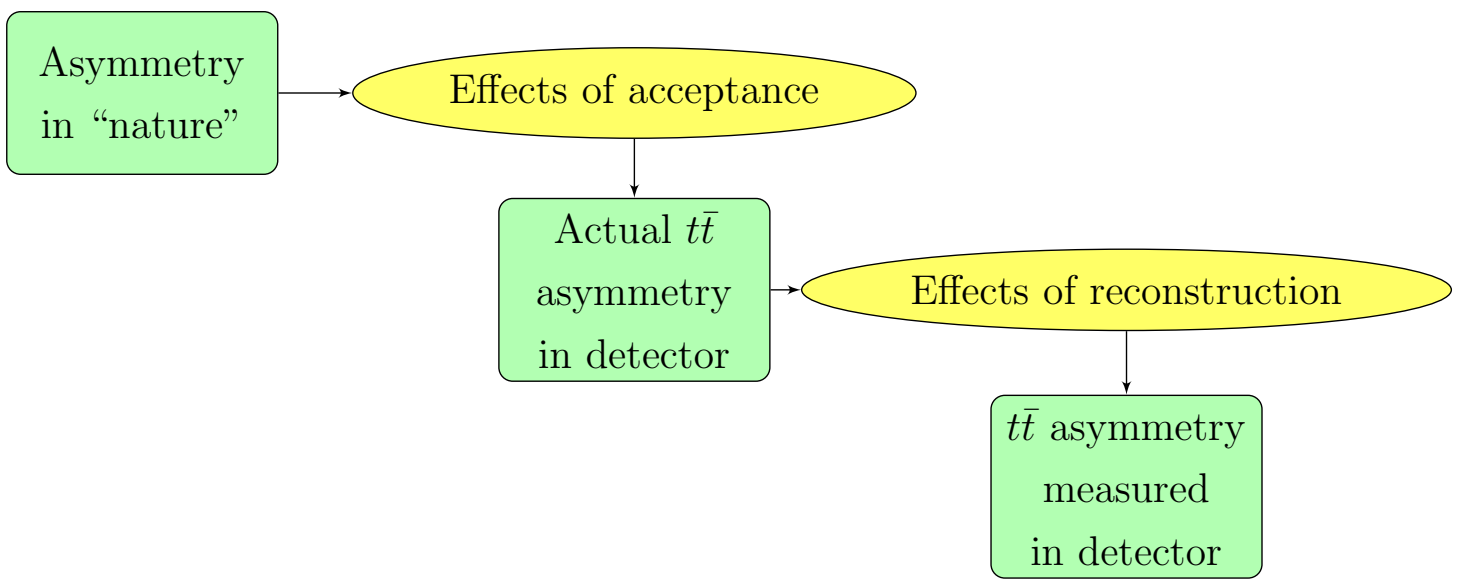

At the current time, the theoretical predictions for the asymmetry have a similar sequence. Because perturbative methods are needed to predict the results from QCD, the actual prediction from the standard model is not known perfectly.

From the theory side, the sequence looks like: 


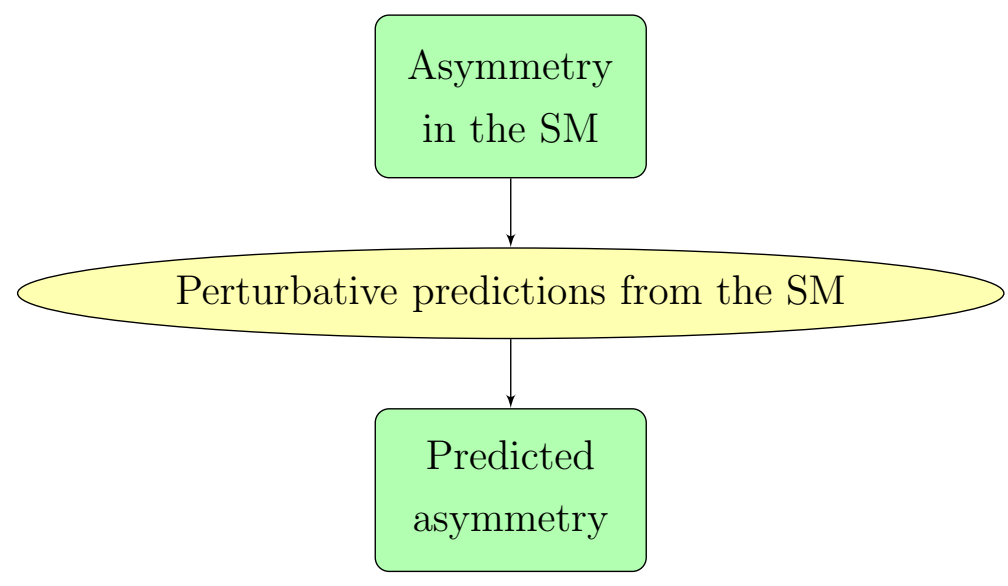

At the end of the day, the goal for the experimentalists and theorists working on this measurement is to answer this question:

\begin{tabular}{|c|c|}
\hline $\begin{array}{c}\text { Asymmetry } \\
\text { in the SM }\end{array} \longrightarrow ? \begin{array}{l}\text { Asymmetry } \\
\text { in "nature" }\end{array}$ \\
\hline
\end{tabular}

\subsection{An analogy}

As an analogy to the $A_{\mathrm{FB}}$ and $A_{\mathrm{FB}}^{l}$ measurements, I present the example of looking at the moon and trying to figure out where the craters are. First imagine a foggy night with a nice telescope. You can see the light of the moon through the fog layer, but everything is blurred out. Luckily, you have a very good theory of the fog and can use adaptive optics to understand how it blurs the light coming in, so you can take the blurry picture and undo the effects from the fog, getting a less blurry picture that shows some detail of the craters. But there is still some blurriness left over, as the effects of the fog cannot be completely reversed. Undoing the effects of the fog is essentially what happens when we correct the $\Delta y$ distribution for the effects of the detector.

Then imagine a nice, clear night. The moon is bright, hanging overhead with a warm glow. But your telescope to view the craters has a red filter over the lens. All other colors of light are blocked by the filter. So you can only see the red parts of the moon. But the shapes of the craters are made out distinctly, with little distortion. When we measure the $q_{l} y_{l}$ distribution, not all of the information from 
the $t \bar{t}$ decay is recorded, but the information that is there is very crisp. Additionally, if we were looking at Mars rather than moon, the red filter might highlight iron deposits on the surface in a way that using a telescope without a filter would not, offering additional information not seen with the standard telescope. In this way, $q_{l} y_{l}$ is an observable that is better resolved and as interesting as $\Delta y$. 


\section{Experimental Apparatus}

For this measurement to take place, two different pieces of scientific equipment are needed: a machine to create pairs of top quarks and another machine to measure the top quark decay products. The machine used to produce top quarks is a particle accelerator called the Tevatron. To identify top quarks and measure their properties, we use a general purpose particle detector called DØ [47].

\subsection{Accelerator}

Located in Batavia, IL at the Fermi National Accelerator Laboratory, or Fermilab, the Tevatron is a proton-antiproton $(p \bar{p})$ collider [48]. In the Tevatron, beams of protons and antiprotons are collided. The center of mass energy at the Tevatron is $1.96 \mathrm{TeV}$ (tera $\left(10^{12}\right)$ electron volts, hence the "Tev"-atron). Each beam is made up into distinct groups of protons and antiprotons called "bunches". During high energy physics operation, the Tevatron collides the bunches at a frequency of 1.4 MHz at the DØ and CDF [49] interaction sites. As shown in Figure 3.1, a sequence of accelerators is used to make bunches protons and anti-protons with energies of $980 \mathrm{GeV}$ : the preacc, the linac, the booster, the main injector and the Tevatron [50].

\subsubsection{Preacc, Linac, Booster and Main Injector}

The first accelerator is the Cockroft-Walton pre-accelerator, or "preacc," an electrically charged dome with a potential of $-750 \mathrm{kV}$. In the pre-accelerator, hydrogen 


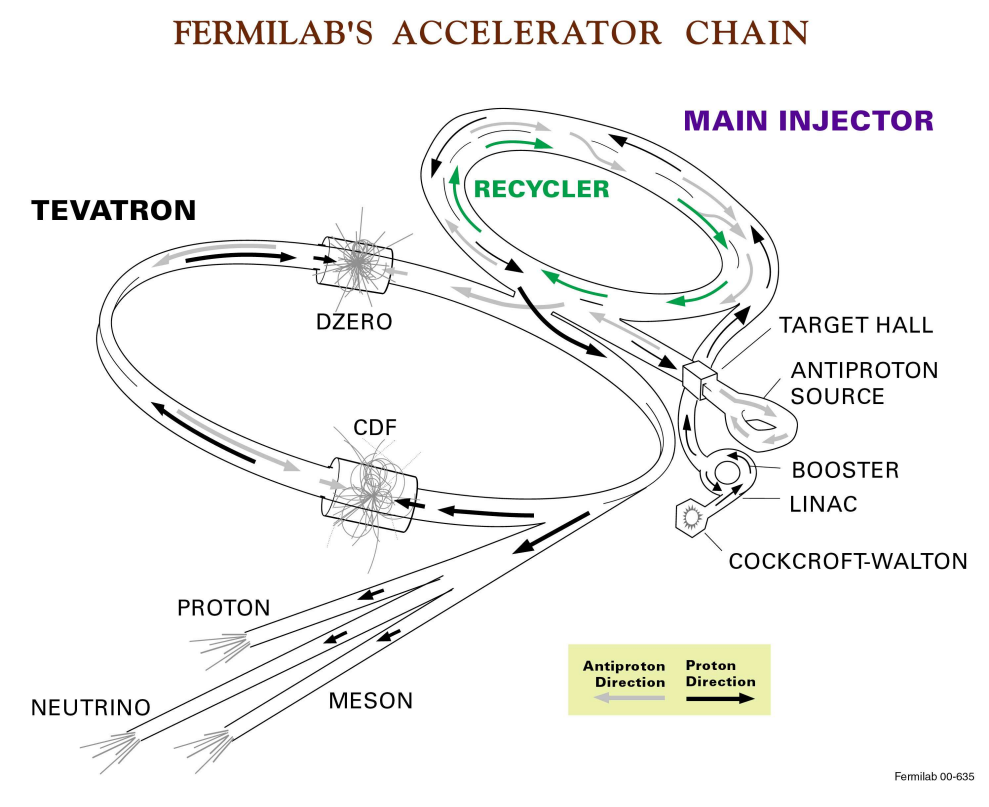

Figure 3.1 The sequence of accelerators that constitute the accelerator chain at Fermilab [51].

gas, $\mathrm{H}_{2}$, is converted into ionized hydrogen gas, $\mathrm{H}^{-}$, and accelerated to an energy of $750 \mathrm{keV}$. The $\mathrm{H}^{-}$ions are transferred to the linac at a rate of $15 \mathrm{~Hz}$.

The next stage is the linear accelerator, or "linac," which increases the kinetic energy of the hydrogen ions a factor of greater than 400 , to $400 \mathrm{MeV}$. The linear accelerator uses a $201 \mathrm{MHz}$ RF signal to accelerate the hydrogen ions, also at a rate of $15 \mathrm{~Hz}$.

After the linac, the booster accelerates the hydrogen ions by a factor of 20 to an energy of $8 \mathrm{GeV}$. A thin foil of carbon strips the electrons from the incoming hydrogen ions, creating protons. The booster is a synchrotron, or circular accelerator, with a $75 \mathrm{~m}$ radius, consisting of magnets and RF cavities. The $8 \mathrm{GeV}$ protons are sent to the main injector.

The main injector (MI), a one kilometer wide synchrotron, is used to accelerate protons to $120 \mathrm{GeV}$ as inputs to the antiproton source and to $150 \mathrm{GeV}$ for injection into the Tevatron, as well as accelerate and store antiprotons for the Tevatron. 


\subsubsection{Antiproton Source}

Protons with an energy of $120 \mathrm{GeV}$ from the main injector are aimed at a nickelbased target, resulting in the creation of two antiprotons with energies of $8 \mathrm{GeV}$ for every 100,000 protons. This process is the most inefficient part of acceleration chain and the largest determiner of the luminosity. Antiprotons undergo a process known as stochastic cooling to reduce the difference in momenta between the antiprotons and decrease the bunch length. The $8 \mathrm{GeV}$ beam of antiprotons is sent to the main injector for storage and accumulation.

\subsubsection{Tevatron}

The Tevatron is housed in a tunnel that is approximately four miles in circumference. The purpose of the Tevatron is to accelerate the incoming proton and antiproton beams from $150 \mathrm{GeV}$ to $980 \mathrm{GeV}$ and collide these beams. More than 1,000 superconducting magnets with fields of $\sim 4 \mathrm{~T}$, cooled by liquid helium, steer the beams of protons and antiprotons. The protons and antiprotons are grouped into "trains" of 12 bunches, spaced 396 ns part. Each bunch is contained in an RF "bucket" of $\approx 18 \mathrm{~ns}$. There are three trains, separated by $2.6 \mu \mathrm{s}$, for a total of 36 bunches. The protons circle clockwise around the ring and the antiprotons circle counterclockwise. There are $2.7 \times 10^{11}$ protons per bunch and $7 \times 10^{10}$ antiprotons

per bunch. The Tevatron has peak luminosities in excess of $400 \times 10^{30} \frac{\mu \mathrm{b}^{-1}}{\mathrm{~s}}$ and on a good week can deliver $70 \mathrm{pb}^{-1}$ of data.

\subsubsection{Recycler}

The Recycler is a ring that stores antiprotons within the Main Injector tunnel. The Recycler was originally designed to recapture antiprotons from the Tevatron at the end of collisions and combine them with antiprotons from the Antiproton Source. This goal proved to be problematic, so the Recycler is instead used to cool the beam of antiprotons further than the Antiproton Source. In addition to stochastic cooling, the Recycler uses electron cooling, where a beam of electrons is overlapped with the beam of antiprotons. The electrons absorb some of the momenta from the antiprotons, making the antiproton bunches more compact, which leads to more collisions and higher luminosity in the Tevatron. 


\section{$3.2 \mathrm{D} \emptyset$ Detector}

Initially designed during the 1980s as a multipurpose particle detector, DØ is wellsuited to measure the daughter products from the decay of heavy particles such as the top quark, and $W, Z$ and Higgs bosons $[52,53,47]$. Shown in Figure 3.2, the $\mathrm{D} \varnothing$ detector has dimensions of 30' $\mathrm{x} 30^{\prime} \mathrm{x} 50^{\prime}$ and weighs approximately 5,000 tons [54]. The purpose of the $\mathrm{D} \varnothing$ detector is to identify particles and precisely measure their energy and momenta using three main systems:

- The tracking system consists of silicon and fiber layers inside a 2T magnetic field. The tracking system is used to identify and measure the momenta of charged particles, to identify interaction vertices and assist with particle identification via displaced vertices. In addition, the tracking system is designed to have a minimal amount of material, so that particles passing through it do not scatter from it and leave the majority of their energy in the calorimeters.

- The calorimetry system uses uranium and liquid argon to identify and measure the energy from neutral and charged particles. The calorimeters are designed to stop most types of particles, except for muons and neutrinos, to help with particle identification.

- The muon system contains drift tubes, scintillators, wire chambers and toroidal magnets, which are used together to identify and measure the momenta of muons. Because muons minimally interact with the other parts of the detector and have long lifetimes, the muon system surrounds the tracking and calorimetry systems.

In the case of a $t \bar{t}$ pair decaying via the lepton + jets channel, electrons and jets are identified by the tracking and calorimetry systems, muons are identified by the tracking, calorimetry and muon systems and the component of the neutrino momenta transverse to the beam pipe are inferred from an imbalance in the momenta of the other particles. 


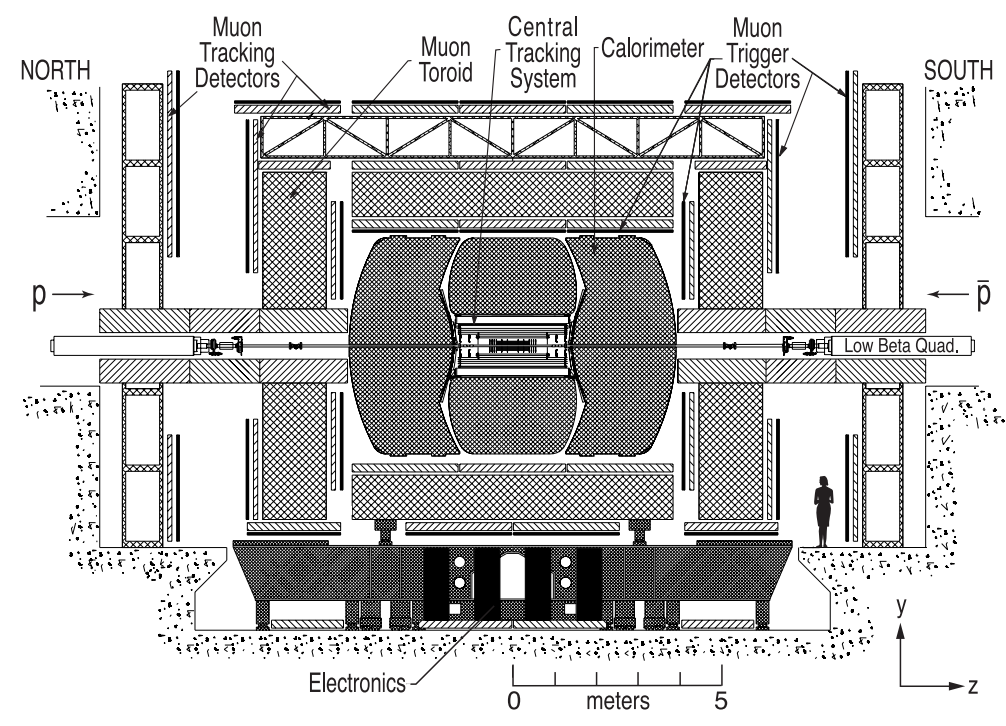

Figure 3.2 Cross section of the $\mathrm{D} \varnothing$ detector as viewed from inside the Tevatron ring, facing outward [47].

\subsubsection{D $\varnothing$ coordinate system}

To ensure that no confusion arises later, in this section the $\mathrm{D} \varnothing$ coordinate system is defined. The standard directions in three-dimensional space are designed such that the $x$ and $y$ axes lie perpendicular to the beam pipe, with the positive $x$ direction pointing along the ground away from the ring and the positive $y$ direction pointing toward the sky, against the force of gravity. The $z$ axis is parallel to the beam pipe, with the positive $z$ direction parallel to the incoming proton momenta and the negative $\mathrm{z}$ direction parallel to the incoming antiproton momenta. The origin, $x=y=z=0$, is at the center of the detector, inside the beam pipe at the nominal interaction point.

A set of modified spherical coordinates, $r, \eta$ and $\phi$, is used. The radial coordinate, $r$, is the distance from the beam pipe, $\sqrt{x^{2}+y^{2}}$, while $\tan (\phi)=\frac{y}{x}$. As shown earlier in Equation 2.4, $\eta=-\log \left(\tan \left(\frac{\theta}{2}\right)\right)$, where $\tan (\theta)=\frac{r}{z}$.

The coordinate system for the particles is not fixed, as the vertex of the main interaction can be up to $60 \mathrm{~cm}$ away from the detector origin, and the magnetic fields bend charged particles, changing their orientation. To account for this difference, two different coordinate systems are used, detector coordinates and physics coordinates. Detector coordinates refer to a specific point in the detector. 


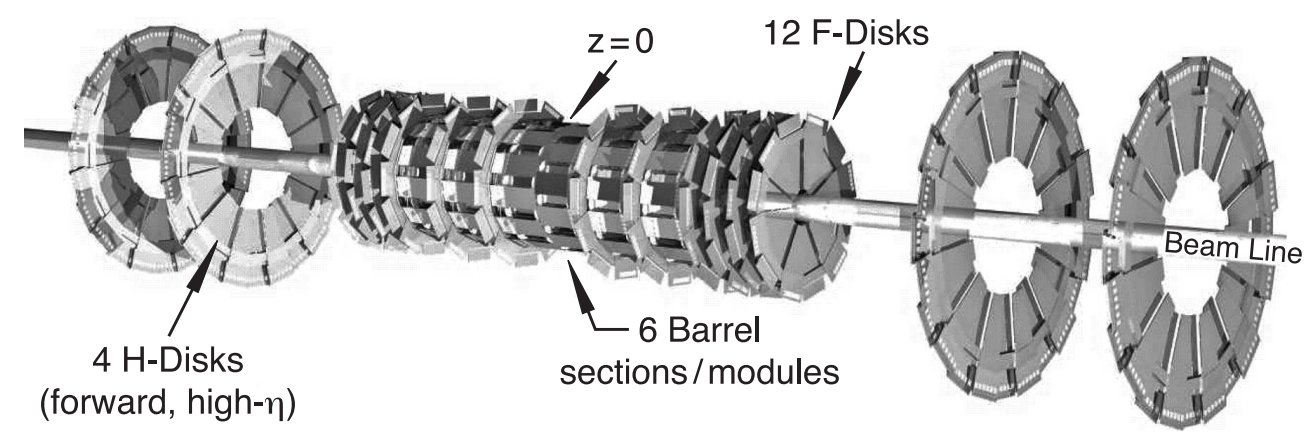

Figure 3.3 Diagram of the SMT [47].

Physics coordinates represent the four-momenta of a particle at the point where the particle is created. In most cases, physics coordinates are used. In situations where detector coordinates are used, the difference will be clearly marked with labels, such as $\phi_{\text {det }}$ and $\eta_{\text {det }}$.

Readers not interested in the detailed workings of the detector can skip to Chapter 4.

\subsubsection{Central Tracking System}

The Central Tracking System is made up of the Silicon Microstrip Tracker (SMT), surrounded by the Central Fiber Tracker (CFT), again surrounded by the $2 \mathrm{~T}$ superconducting solenoid magnet. Figure 3.4 shows the tracking system, with the SMT closest to the beam pipe, followed by the CFT and then the solenoid magnet. The central tracking system is designed to measure the position of the main interaction vertex, i.e. where the $t \bar{t}$ pair is produced, with a resolution of $\sim 35 \mu \mathrm{m}$, and to measure the impact parameter of tracks with a resolution of $15 \mu \mathrm{m}$ to assist in the identification of jets from $b$ quarks. The precision of the tracker also allows for good lepton and jet transverse momentum resolution, as well as the measurement of missing transverse energy, $\mathbb{E}_{\mathrm{T}}$. In addition, the tracking system is crucial for particle identification. Charged particles such as electrons and muons leave single tracks. Photons, neutrons and other neutral particles, such as $\pi^{0}$ mesons, leave no tracks. Jets from quark decay leave groups of tracks that initiate at the point of decay. 


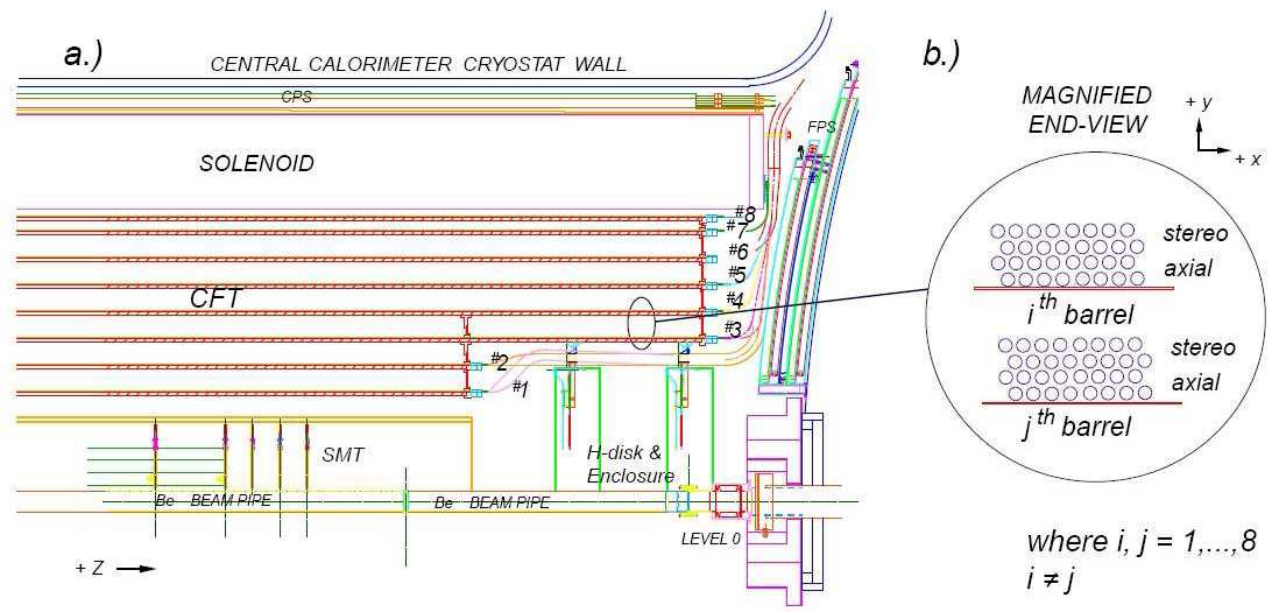

Figure 3.4 Diagram of the tracking system, with focus on the CFT.

The SMT consists of $300 \mu \mathrm{m}$ thick silicon wafers lined with charge collecting strips separated by a distance (pitch) of $50 \mu \mathrm{m}$ [55]. Due to the long interaction region $\left(\sigma_{z}=25 \mathrm{~cm}\right)$, the SMT has sections that are both parallel (barrel sections) and perpendicular (disk sections) throughout its $2.4 \mathrm{~m}$ length, as seen in Figure 3.3. There are six barrel sections, twelve F-disk sections and four H-disk sections in the SMT. The barrel sections have four different layers of silicon microstrip detectors. To measure additional tracking information, the four layers of the SMT barrels are double sided, with one set of strips parallel to the beam pipe (axial) and another set of strips at angles of either $2^{\circ}$ or $90^{\circ}$ to the axial side (stereo). Two layers in the outer barrels are single-sided with axial strips. The F-disk sections are disks made up of twelve double-sided wedge-shaped detectors with $30^{\circ}$ of stereo angle. Each H-disk is made up of twenty four wedges each with a pair of single sided wedge detector mounted back-to-back, giving the pair an effective stereo angle of $15^{\circ}$. An additional layer of silicon situated between the beam pipe and layer 1 of the SMT, known as layer 0, was added to the detector in 2005 [56]. This new layer further improves vertex resolution.

Eight concentric cylinders set at a distance of $20 \mathrm{~cm}$ to $52 \mathrm{~cm}$ from the center of the beam pipe make up the CFT [57]. As seen in Figure 3.4, each cylinder is made up of a doublet layer of scintillating fiber parallel to the beam pipe and another doublet layer of fiber that is at an angle of $+3^{\circ}$ or $-3^{\circ}$ with respect to the axial layer. The orientations of the stereo double layers alternate between the two 
angles. Each doublet layer is made up of two overlapping layers of $835 \mu \mathrm{m}$ thick fiber in parallel to each other.

Designed to optimize the momentum resolution $\frac{\delta p_{T}}{p_{T}}$, the superconducting solenoid magnet is $2.73 \mathrm{~m}$ in length and $1.42 \mathrm{~m}$ at the outer diameter [58]. The solenoid is made up of niobium-titanium and copper wire cooled to a temperature of 4.7 $\mathrm{K}$ by liquid helium. The solenoid provides a uniform $1.9 \mathrm{~T}$ magnetic field parallel to the beam pipe that causes particles passing through the tracking detectors to change their direction and curve as they move away from the beam pipe. The field configuration is shown in Figure 3.5. The radius of curvature in meters, $R$, for a particle track is

$$
R=\frac{p_{T}}{0.3 q B}
$$

where $p_{T}$ is the transverse momentum of the particle, $B$ is the magnetic field strength in Tesla and $q$ is the charge of the particle in units of the charge of an electron. The momentum of a particle being detected is not known before detection and is measured with the radius of curvature of the track.

\subsubsection{Preshower Detector}

The central preshower detector (CPS) surrounds the solenoid magnet and the forward preshower detectors (FPS) lie between the end of the CFT and the beginning of the end calorimeter. As shown in Figure 3.6, the preshower detectors use triangular scintillating fiber with a wavelength-shifting fiber down the center. The preshower detectors aid in photon and electron identification, as well as assisting in the background rejection of hadronic particles.

The CPS has three concentric cylinders of scintillating fibers. In between the scintillating fibers and the solenoid is a layer of lead. The fibers in the concentric cylinders are arranged such that the fibers of the first layer are parallel to the beam pipe and then the following layers have orientations of $24^{\circ}$ and $-24^{\circ}$ with respect to the first layer. The combination of material from the solenoid and the layer of lead creates approximately two to four radiation lengths of material before the calorimeter, depending on $\eta_{\text {det }}$. A radiation length, $X_{0}$, is the mean distance 


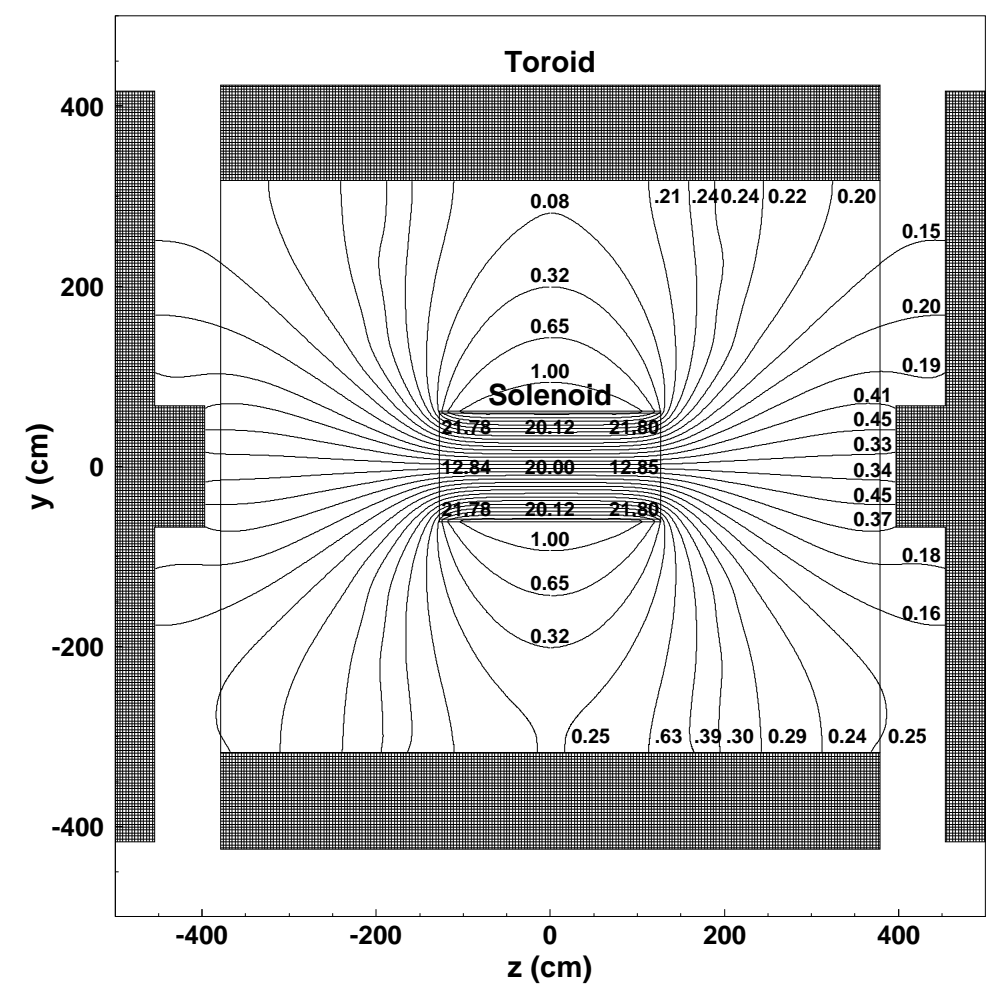

Figure 3.5 Diagram of the solenoid and toroid magnet fields used in the D $\varnothing$ detector [47]. The units for the magnetic fields are $\mathrm{kG}$.

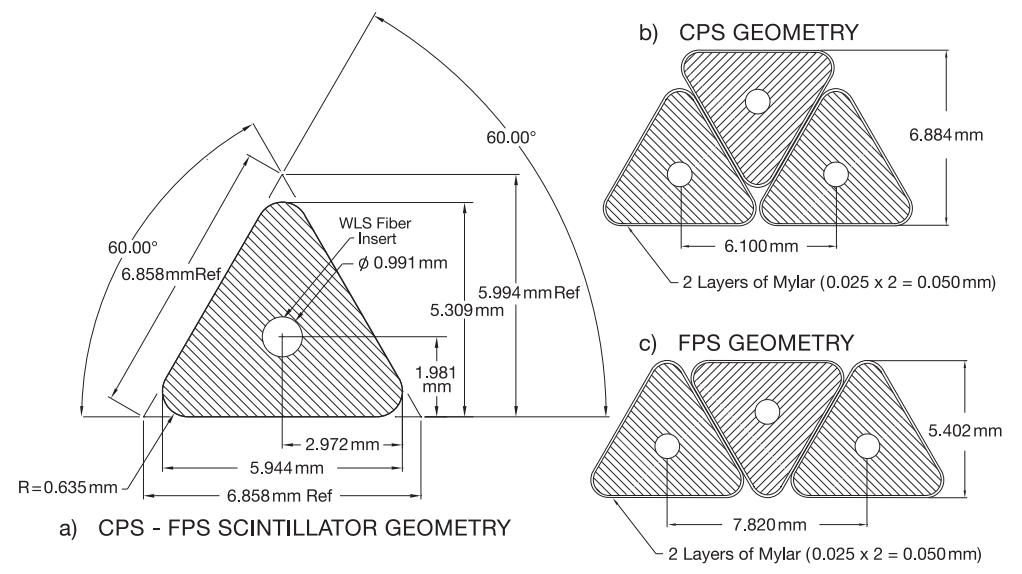

Figure 3.6 a) Cross section of the triangular scintillator used in the preshower, with the wavelength-shifting fiber shown in the center. b) Geometry of scintillator stacking in the CPS. c) Geometry of scintillator stacking in the FPS [47]. 


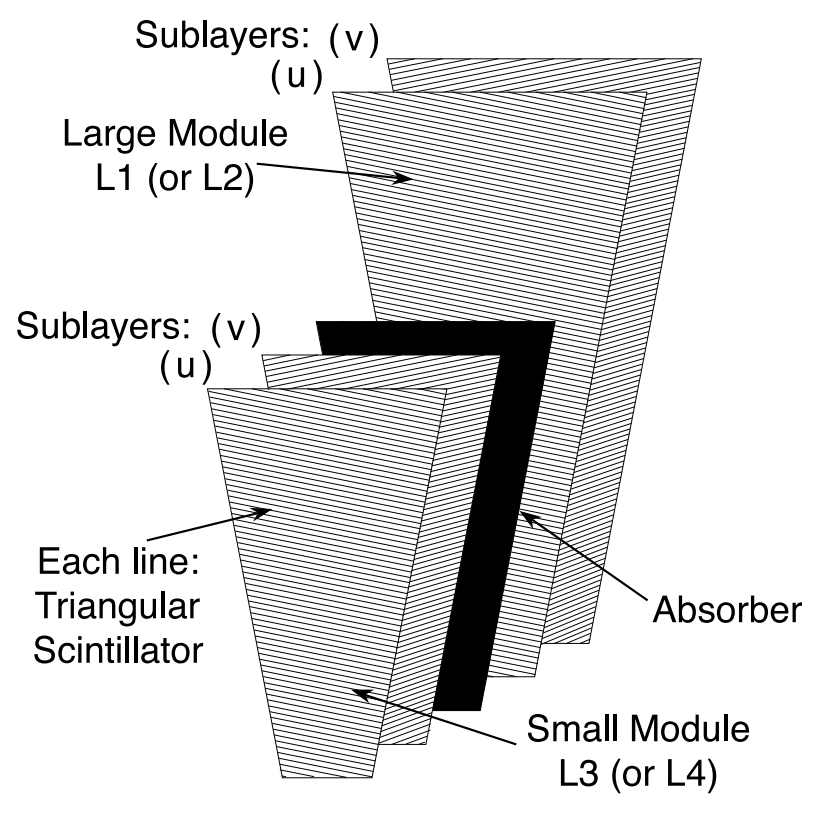

Figure 3.7 Diagram of an example wedge from the FPS showing the stereo angle of the fibers [47].

that a high energy electron travels through a material before losing all but $\frac{1}{e}$ of its initial energy.

The FPS detectors are made up of 16 wedges each. Each wedge is made of two layers of scintillator, followed by two layers of lead and stainless steel absorber material, followed by two more layers of scintillator, as shown in Figure 3.7. The layers of scintillator are oriented with an angle of $22.5^{\circ}$ with respect to each other. Electrons will generally pass through in the first two layers with minimal interaction, allowing the location information to be combined with the information from the tracker for greater resolution. However, electrons will typically shower in the absorber layer, while charged hadronic particles will not, allowing for electron identification.

\subsubsection{Calorimetry System}

The calorimeter is made primarily of uranium and liquid argon [52]. The calorimetry system is used to identify and measure the energy of electrons, photons, neutrons and jets created by quarks and gluons, which decay as showers of hadrons, as well as to measure the missing transverse energy and aid in muon identifica- 
tion. As seen in Figure 3.8, there are three sections of calorimeter. The central calorimeter $(\mathrm{CC})$ is arranged concentrically around the solenoid magnet with coverage out to $|\eta|<1$, and the end calorimeters (EC) are situated at the ends of the solenoid magnet, with coverage out to approximately $|\eta|<4$. The three calorimeter sections are housed in separate cryostats, which keep the calorimeters at a temperature near $90 \mathrm{~K}$.

Alternating sections of absorber material (uranium) and active material (liquid argon) make up the calorimeter. This segmentation allows energy deposits to be sampled at different depths, assisting in shower shape measurement and particle identification. The electromagnetic (EM) section of the calorimeters is the first four readout layers, which are designed to measure and fully absorb the energy of electrons and photons. In the $\mathrm{CC}$, three separate readout layers make up the fine hadronic $(\mathrm{FH})$ section and the last readout layer is the course hadronic $(\mathrm{CH})$ section. The EC hadronic sections are slightly more complex, with inner fine hadronic, inner course hadronic, middle fine hadronic, middle course hadronic and outer course hadronic sections. The readout layers are made up of cells which measure $0.1 \times 0.1$ in $\eta$ and $\phi$. The third EM layer is segmented twice as finely at $0.05 \times 0.05$ in $\eta$ and $\phi$, to assist in electron ID. Figure 3.9 shows that the cells are stacked in towers with dimensions of $0.1 \times 0.1$ in $\eta$ and $\phi$.

Figure 3.10 shows an example readout cell from the calorimeter. Each cell is made up of layers of grounded absorber material and signal boards with a high voltage up to $2 \mathrm{kV}$. Liquid argon fills the gaps between the absorber material and the signal boards. The interaction of the liquid argon with charged particles from electromagnetic or hadronic showers will cause it to ionize, sending a current to the signal boards. In the EM section, depleted uranium is used as the absorber material. Uranium-niobium is the absorber material in the FH section, and the $\mathrm{CH}$ section has copper for the absorber.

The calorimeters have two different measures of radiation density: $X_{0}$, the radiation length for an electron, described earlier, and $\lambda_{A}$, the nuclear absorption length, which is the amount of material needed to absorb $\left(1-\frac{1}{e}\right)$ of the energy of a hadronic particle, such as a pion. In most materials, $\lambda_{A}$ is much longer than $X_{0}$. The EM section of the calorimeter has $X_{0}=20.5$ and $\lambda_{A}=0.76$. The three read out cells making up the $\mathrm{FH}$ section each have $\lambda_{A} \approx 1$ and the $\mathrm{CH}$ section has $\lambda_{A}=3.2$. The numbers of nuclear interaction lengths in the EC sections are 
slightly larger.

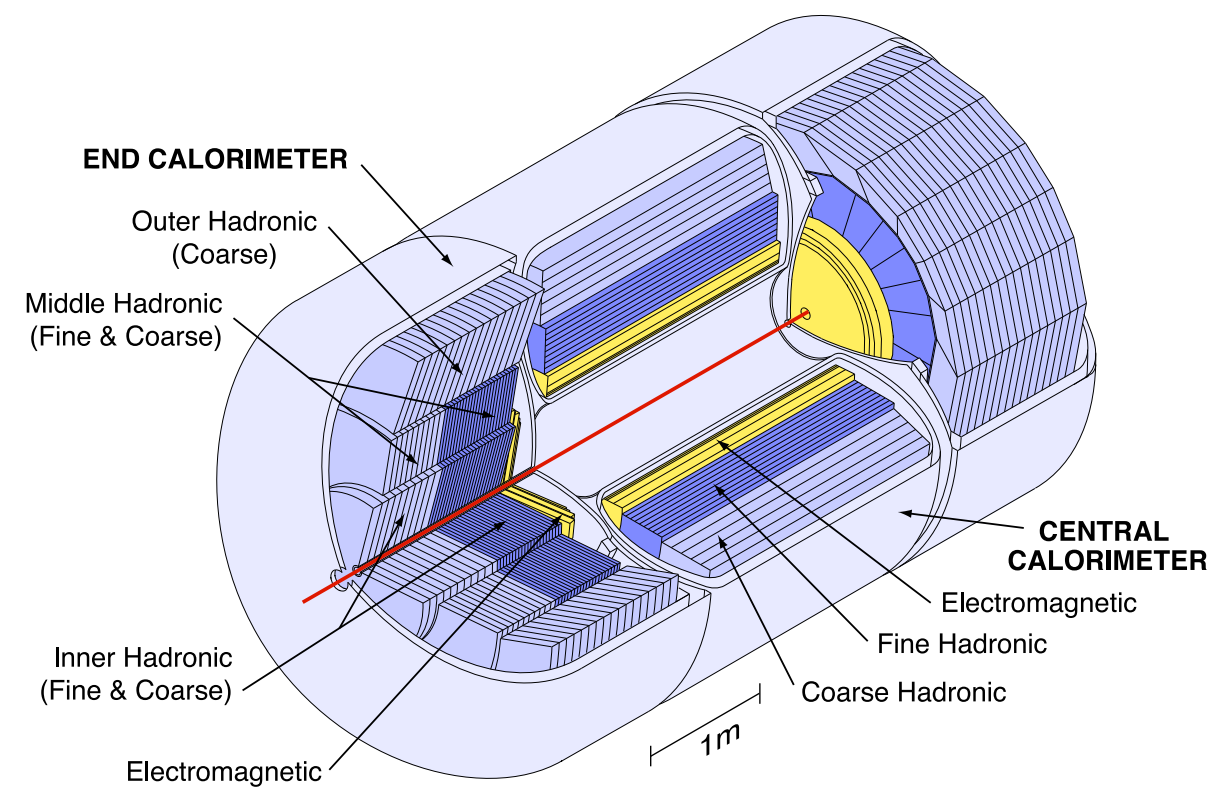

Figure 3.8 Cut-away view of the D $\varnothing$ calorimeter [47].

When highly energetic electrons or photons interact with the calorimeter, they will lose energy via bremsstrahlung and pair production, creating an electromagnetic shower. When the energies of the electrons in the shower are reduced to below a threshold value, the electrons ionize the liquid argon, rather than continuing to emit bremsstrahlung, causing the shower to cease. The overall length of the shower depends on the material properties of the absorber, mainly density. Because the EM section of the calorimeter has $X_{0}=20.5$, almost all of the energy from electrons and photons are deposited in the EM section, as shown in Figure 3.11.

When quarks or gluons decay into cone-shaped jets, many of the particles are hadrons, which interact with the detector via the strong force. The hadrons scatter off heavy nuclei as they propagate through the calorimeter, creating a hadronic shower. Because the nuclear interaction length is typically much longer than the radiation length, hadronic showers are much longer in extent than electromagnetic showers, and may engulf a large part (in $r$ ) of the hadronic calorimeter.

The calorimeter has approximately 50,000 readout channels from all of the cells. With so many cells, the likelihood of a false signal from electronic noise 


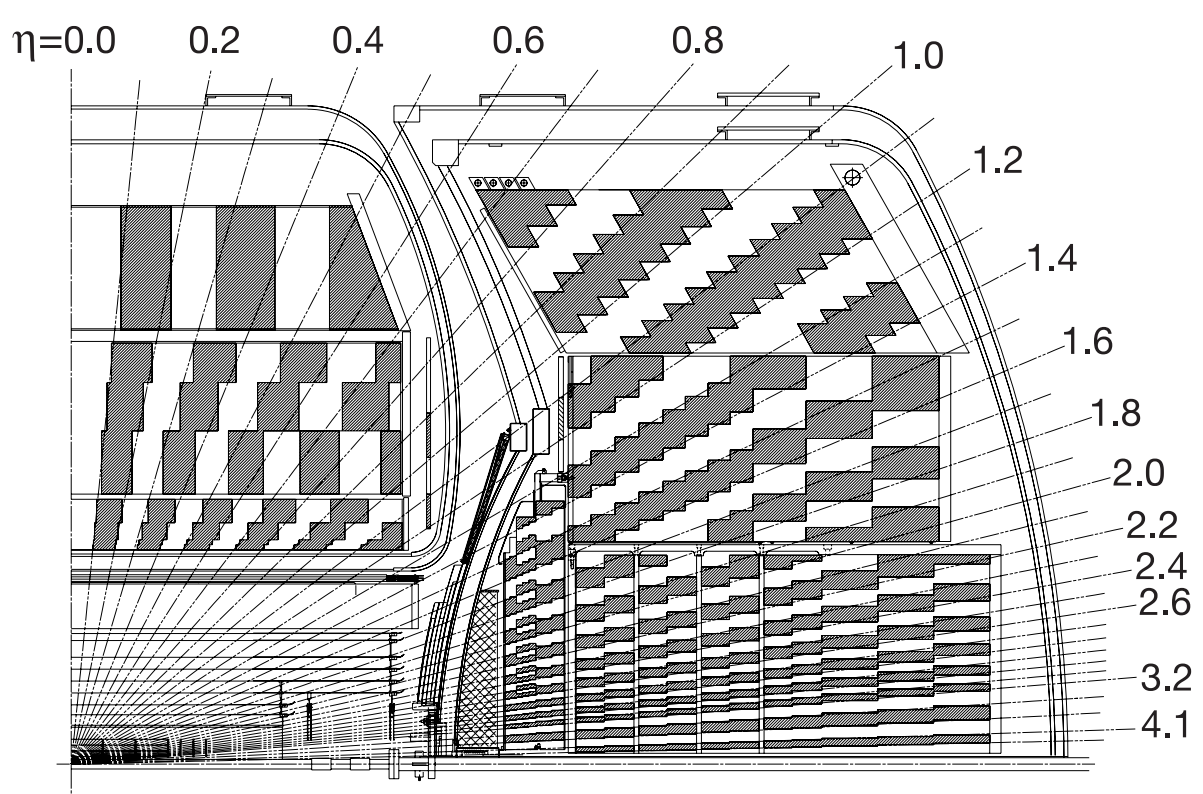

Figure 3.9 Cross section of the calorimeters showing $\eta[47]$.

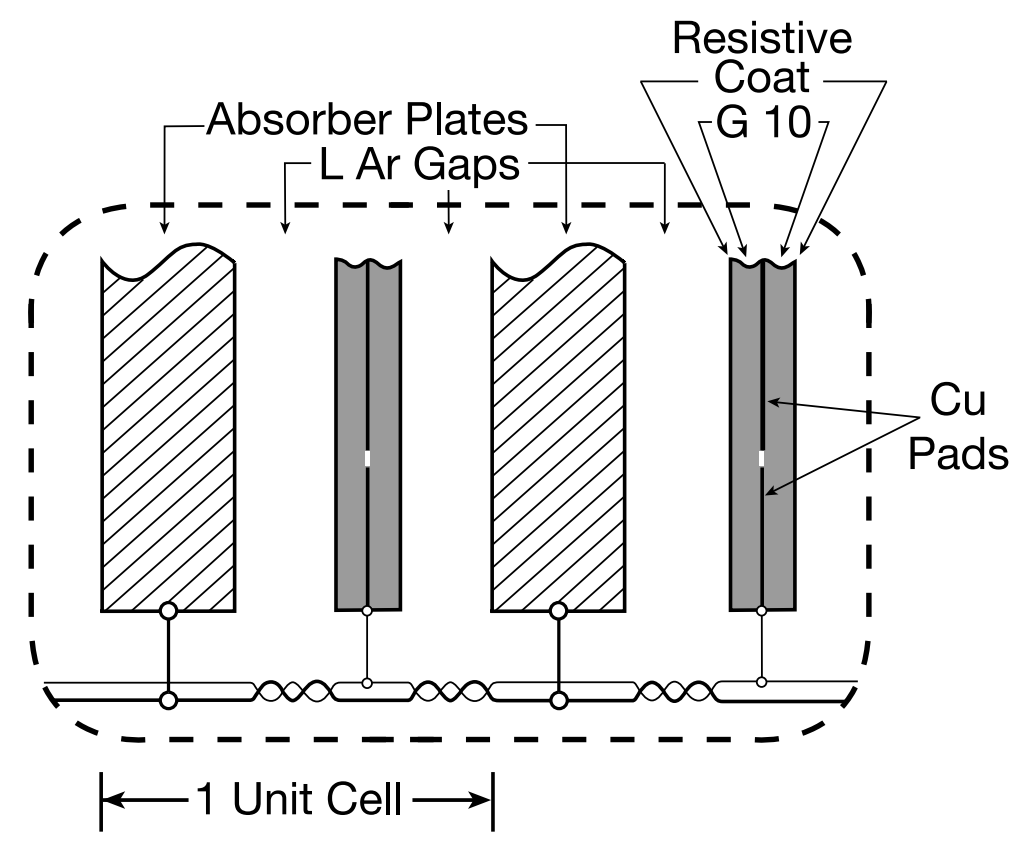

Figure 3.10 Schematic diagram of an individual calorimeter cell [47]. 


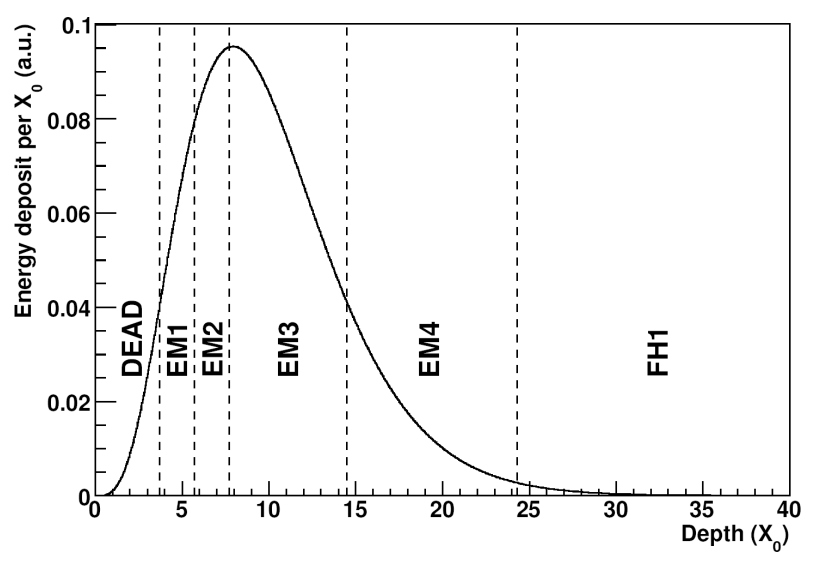

Figure 3.11 Simulated energy deposition spectrum of a shower initiated by a $45 \mathrm{GeV}$ electron, overlaid with the different sections of the calorimeter.

or the decay of a uranium atom is not negligible. For this reason, the "T42" zero-suppression algorithm is used, and cells that do not measure energy deposits that are significantly above the noise level ( $4 \sigma$ for a standalone cell, or $2.5 \sigma$ for a cell next to a cell with $4 \sigma$ ) are not sent to the reconstruction algorithms [59]. Zero-suppression helps to properly measure $\mathbb{E}_{\mathrm{T}}$, as well as other quantities.

Since there are gaps between the calorimeters from $0.8<|\eta|<1.4$, an additional detector known as the inter-cryostat detector (ICD) is used to ensure no charged particles escape detector. The ICD is made up of 0.5" thick tiles of scintillating material attached to the exterior of the cryostats encompassing the EC between $1.1<|\eta|<1.4$. In addition, single calorimeter cells known as massless gaps are placed in front of the uranium absorbers inside of the central and end cryostats. These additional detectors aid in the sampling of electromagnetic and hadronic showers and allow continuity between the cryostats for that portion of $|\eta|$.

\subsubsection{Muon System}

Muons are the only type of particle (besides neutrinos) to pass all of the way through calorimetry system with a high probability. Muons at the energies from typical $t \bar{t}$ decays, $\sim 20 \mathrm{GeV}$ to $\sim 100 \mathrm{GeV}$, are minimum-ionizing particles (MIP), and they will only deposit approximately $0.25 \mathrm{GeV}$ of energy for every nuclear interaction length of material they cross. Because muons escape the dense material 


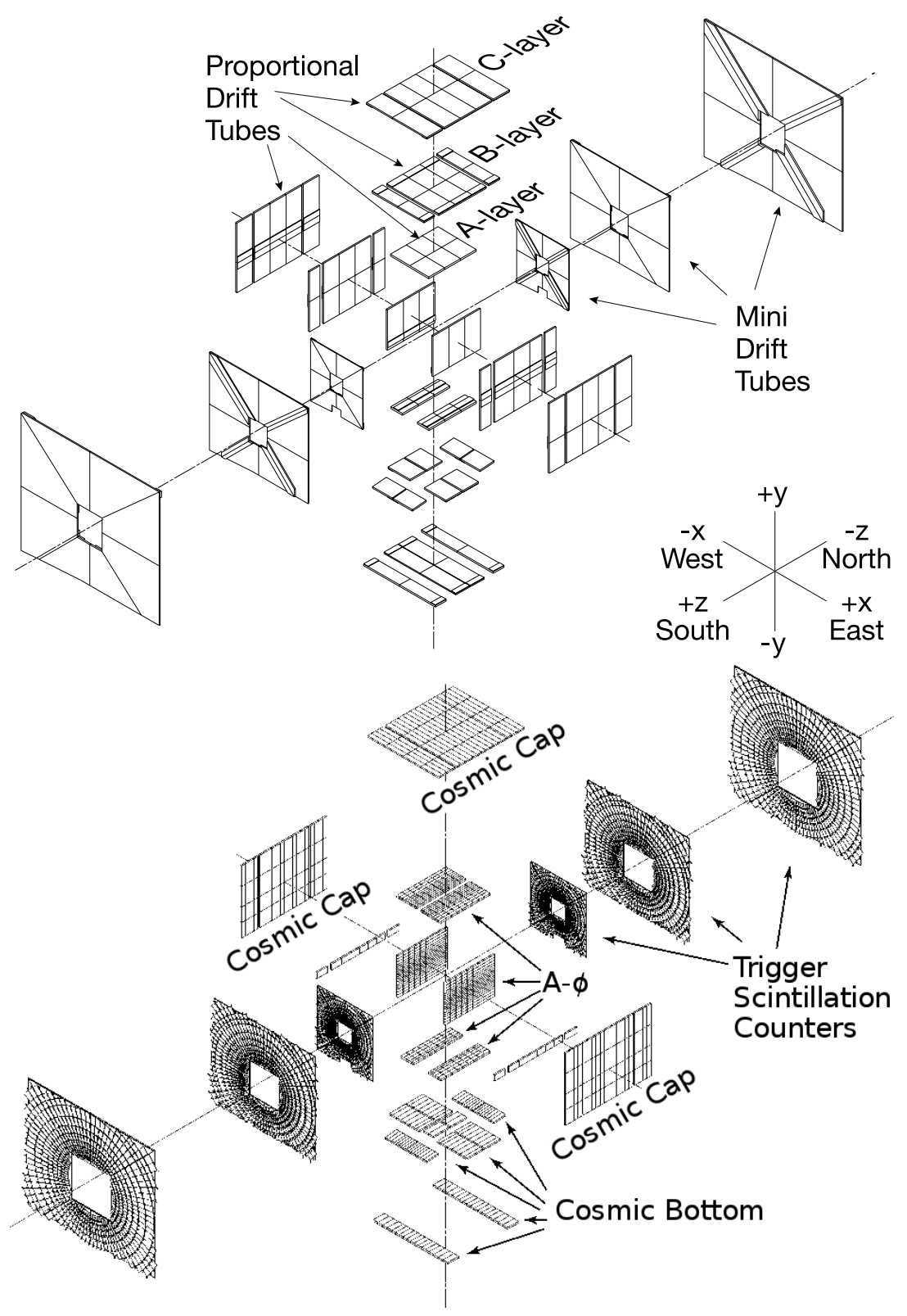

Figure 3.12 View of the drift tubes (top) and scintillation counters (bottom) in the muon system [47]. 
of the absorbers, a layer of detectors known as the muon system surrounds the calorimeters.

The central muon system, with coverage up to $|\eta| \lesssim 1.0$, consists of a set of toroid magnets (see Figure 3.5), proportional drift tubes (PDT) and central scintillation counters [52]. The forward muon system continues coverage up to $|\eta| \approx 2.0$ and contains another set of toroid magnets, mini drift tubes (MDT) rather than PDTs and trigger scintillation counters. Figure 3.12 shows the "exploded" view of the different parts of the muon system.

The central muon has three layers, A, B and C, with layer A lying closest to the beam pipe, inside of the toroid magnets. Layers $B$ and $C$ are outside of the 1.9 T toroid magnets. Four decks of PDTs make up the panels of layer A, while layers B and C each have three decks, as seen in Figure 3.13. The individual PDTs are $10 \mathrm{~cm}$ wide and 5.5 thick with copper cathode pads on the top and bottom walls, and have gold-plated tungsten anode wires running down the middle. The cathode pads are kept at a voltage of $+2.3 \mathrm{kV}$ and the anode wires have a voltage of $+4.56 \mathrm{kV}$. The PDTs contain a gas mixture of $84 \%$ argon, $8 \%$ methane and $8 \%$ $\mathrm{CF}_{4}$, optimized for faster drift times for the high luminosity environment of Run II.

The toroid magnets bend the muons in the $\eta$ direction, allowing the muon momentum to measured from the curvature of the track. Because the tracking system is more precise and the muons have lost a few GeV of energy by passing through the calorimeter, the muon system is mostly used for muon identification. In the case where muons have transverse momenta greater than $100 \mathrm{GeV}$, the muon system provides a much longer path than the tracker for determining the radius.

When a muon passes through the PDTs, it ionizes the gas, leaving a trail of electrons. These electrons drift toward the anode, creating a cascade of charge as more gas is ionized during the drift. The farther muons are from the anode, the greater the amount of charge that reaches the anode.

Layers of scintillators known as the cosmic cap and cosmic bottom sit on the outside of layer $\mathrm{C}$ of the PDTs. These layers have a fast response time to ensure the muons are associated with the appropriate bunch crossing and act as a veto against muons from cosmic rays. Another layer of scintillator, known as the $A \phi$ 
layer, covers layer A. The $A \phi$ layer provides fast detection, which is primarily used for triggering on muons and muon identification.

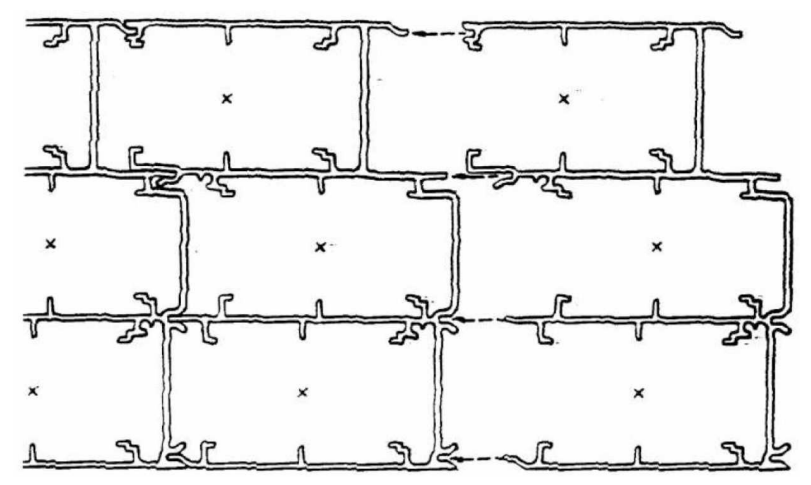

Figure 3.13 Side view of the PDTs used in the central muon system [47].

The forward muon system is very similar to the central muon system, with the PDTs replace by MDTs. The MDTs consist of eight cells that measure $9.4 \mathrm{~mm}$ x $9.4 \mathrm{~mm}$, as shown in Figure 3.14. The gas mixture in the MDTs is $90 \% \mathrm{CF}_{4}$ and $10 \%$ methane. Like the central muons system, four planes of MDTs make up layer A of the forward muon system, situated inside the toroid magnets and three planes of MDTs make up layers B and C, which are outside of the toroid magnets. These layer also contain planes of scintillator detectors, used for triggering and background rejection. Figure 3.15 shows the concentric circular arrangement of the scintillator panels in the forward muon system.

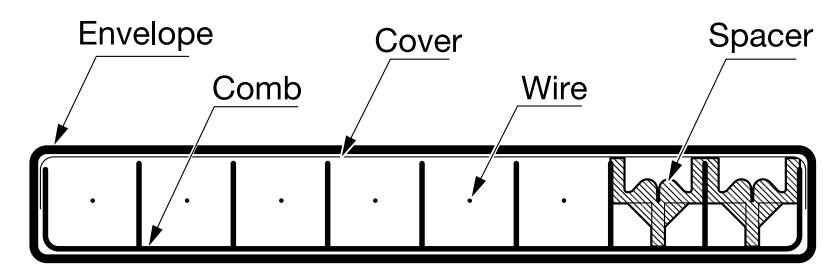

Figure 3.14 Cross section of the eight cells making up an MDT [47].

\subsubsection{Luminosity Monitor}

The luminosity monitor is needed to measure the instantaneous luminosity to choose the correct set of triggers, as well as to calculate the integrated luminosity 


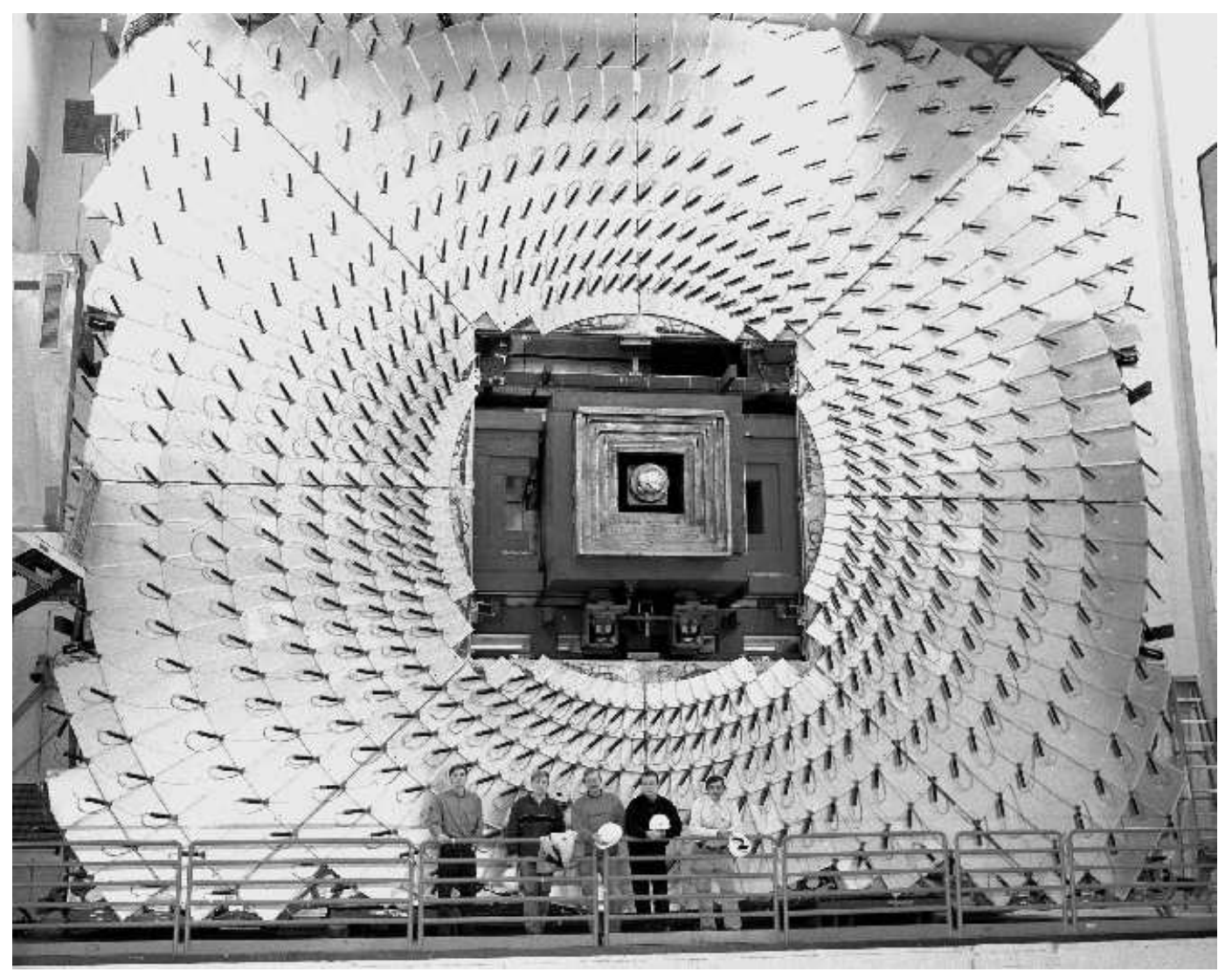

Figure 3.15 Picture of the scintillator panels in layer $\mathrm{C}$ of the forward muon detector [47]. 
for cross section measurements. The luminosity monitor essentially counts the number of inelastic $p \bar{p}$ collisions in a time interval of $60 \mathrm{~s}$, known as a luminosity block, to determine the luminosity. The luminosity blocks are short enough that the instantaneous luminosity does not vary much over the time interval.

Two luminosity detectors sit at $\pm z=140 \mathrm{~cm}$. Shown in Figure 5.1, each luminosity detector is made up of an array of twenty-four $15 \mathrm{~cm}$ long scintillating counters which use PMTs as readouts. The luminosity detectors cover an area in rapidity of $2.7<|\eta|<4.4$. After a collision, the remnants of the $p \bar{p}$ system that are not involved in the hard interaction travel down the beam pipe with little transverse momentum. The luminosity detectors count the number of these remnants.

The instantaneous luminosity, $\mathcal{L}$, is determined using the following formula [60]:

$$
\mathcal{L}=\frac{1}{\epsilon \times A \times \sigma_{p \bar{p}}} \frac{d N}{d t}(p \bar{p})
$$

where $\epsilon$ is the efficiency of the luminosity detector, $A$ is the acceptance of the luminosity detector and $\sigma_{p \bar{p}}$ is the inelastic cross section. The $z$ coordinate of the interaction vertex is found from the difference in the time-of-flight measurement from the two luminosity detectors, with a resolution of about $6 \mathrm{~cm}$.

\subsection{Trigger}

The rate of collisions at $\mathrm{D} \varnothing$ is on average approximately $1.7 \mathrm{MHz}^{1}$. As each event needs to be recorded for further analysis and takes up disk space, it is impossible to record the information from 1.7 million crossings every second. Figure 3.17 shows the cross sections for different physical processes at the Tevatron. The phenomena that the Tevatron was built to study, such as $W$ and $Z$ boson production, $t$ quark production, and $b$ quark physics, are rare compared to the total cross section, which the luminosity monitor uses. The trigger is the mining pan that helps experimenters search for particle physics gold. When a given event looks

\footnotetext{
${ }^{1} 12$ collisions every $396 \mathrm{~ns}$ with a long break of $2600 \mathrm{~ns}$ leads a length $7300 \mathrm{~ns}$ for every 12 bunch crossings, or a bunch crossing every $600 \mathrm{~ns}$.
} 


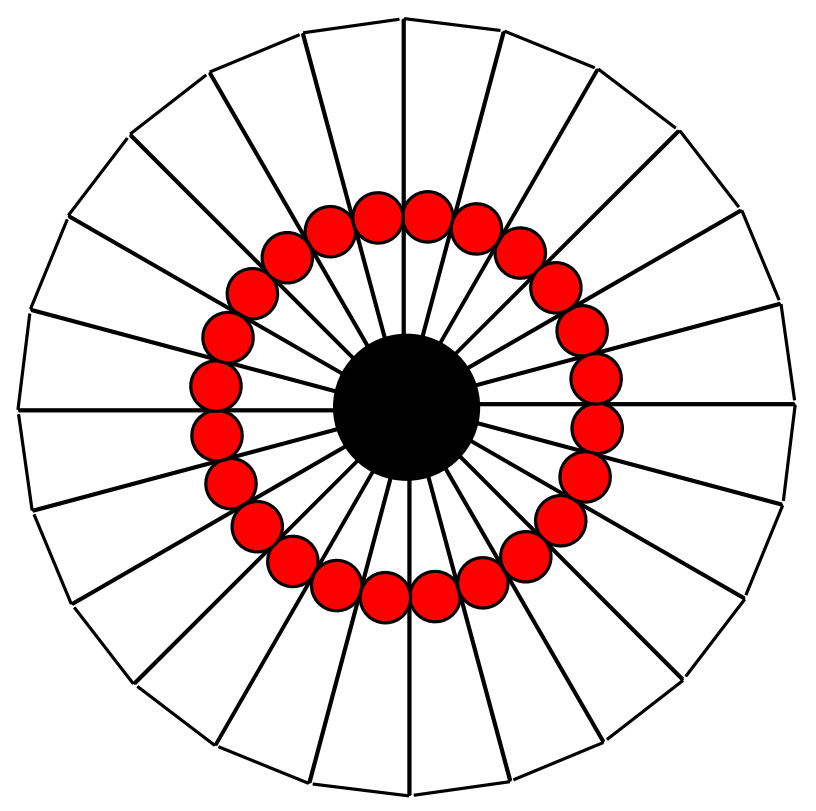

Figure 3.16 Diagram of the luminosity monitor, with the 24 PMTs displayed at red circles [47].

interesting, where interesting is defined by experimenters, the trigger is pulled and the event is recorded.

In the case of $\mathrm{D} \varnothing$, interesting events are characterized by their decay patterns in the detector. To study electroweak physics, $W$ and $Z$ bosons are detected by high $\mathrm{p}_{\mathrm{T}}$ leptons or high $\mathrm{p}_{\mathrm{T}}$ jets. Top quark decays might produce high $\mathrm{p}_{\mathrm{T}}$ leptons and high $\mathrm{p}_{\mathrm{T}} b$ jets. The triggers are designed to specifically search for these types of signatures. At the end of the day, the trigger decides which signatures in the detector will be kept, and by proxy the types of physical processes will be passed on to experimenters for further study, and which ones will be discarded.

The D $\varnothing$ trigger consists of three levels, shown in Figure 3.18 [62]. Higher levels are more complex and take inputs from the lower levels of the trigger. Events that are stored for further analysis must pass all three levels. The Level 1 trigger is made up of hardware elements in each detector subsystem that run fast tests (3.5 $\mu \mathrm{s})$ to see if patterns of hits in the tracker or energy deposits in the calorimeter are left by energetic particles. The results from each Level 1 subsystem are combined to make the final Level 1 decision. Readout of the information sent to the Level 2 trigger takes approximately $10 \mu \mathrm{s}$, so the maximum Level 1 acceptance rate is 


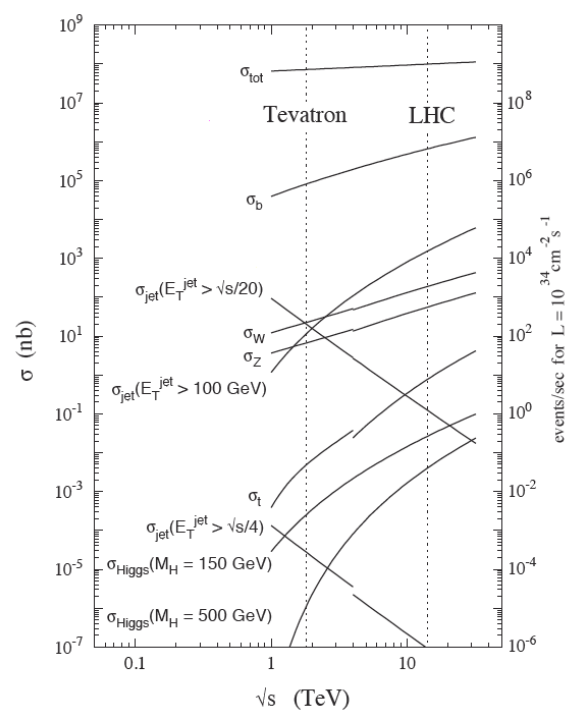

Figure 3.17 Cross sections for different processes at different colliding energies. The dicontinuities occur because of the difference between the colliding particles at the Tevatron $(p \bar{p})$ and the LHC (pp) [61].

$5 \mathrm{kHz}$.

Events that pass the Level 1 trigger are sent to the Level 2 trigger, which is made up of preprocessors which use more detailed information from each of the subsystems. These in turn feed into a global Level 2 trigger, which combines the information into physics "objects" such as electrons, muons and jets at a rate of $100 \mu \mathrm{s}$. The maximum acceptance rate of the Level 2 trigger is $1 \mathrm{kHz}$.

Events passing the Level 1 and Level 2 triggers are sent to the Level 3 trigger system, which consists of a farm of a few hundred computers that are programed to reconstruct each event in $25 \mathrm{~ms}$. Although the time frame is short, the reconstruction algorithms used by the Level 3 trigger are very close in complexity to the algorithms used by offline analyses. Events accepted by the Level 3 trigger are stored on tape. Depending on the luminosity, the Level 3 output rate runs in the range of $50 \mathrm{~Hz}$ to $200 \mathrm{~Hz}$. Larger Level 3 acceptance rates may cause issues with further computing downstream in the data flow.

When protons and antiprotons start colliding after being loaded into the Tevatron, the luminosity is very high and quickly decays to lower values, as seen in Figure 3.19. During the higher luminosity portions of the data taking, many more 
events with interesting features are produced than during periods with lower luminosities. For this reason, there are trigger settings called "pre-scales" that are designed to only accept a certain fraction of events from each type of level 1 trigger for different ranges of luminosity. Pre-scales designed for higher luminosities allow lower fractions of events to be triggered than the lower luminosity pre-scales. The pre-scales are designed to keep the Level 3 output rate constant.

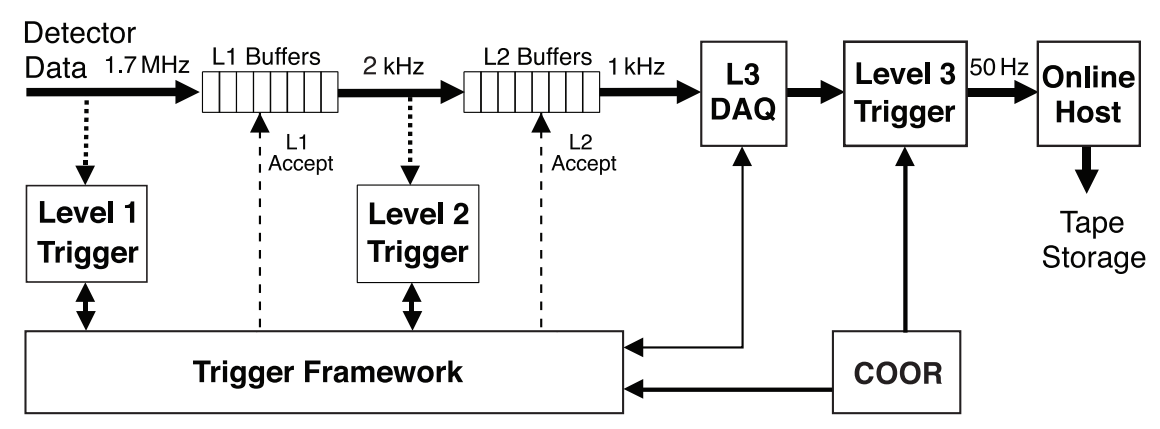

Figure 3.18 Diagram of the trigger framework [47].

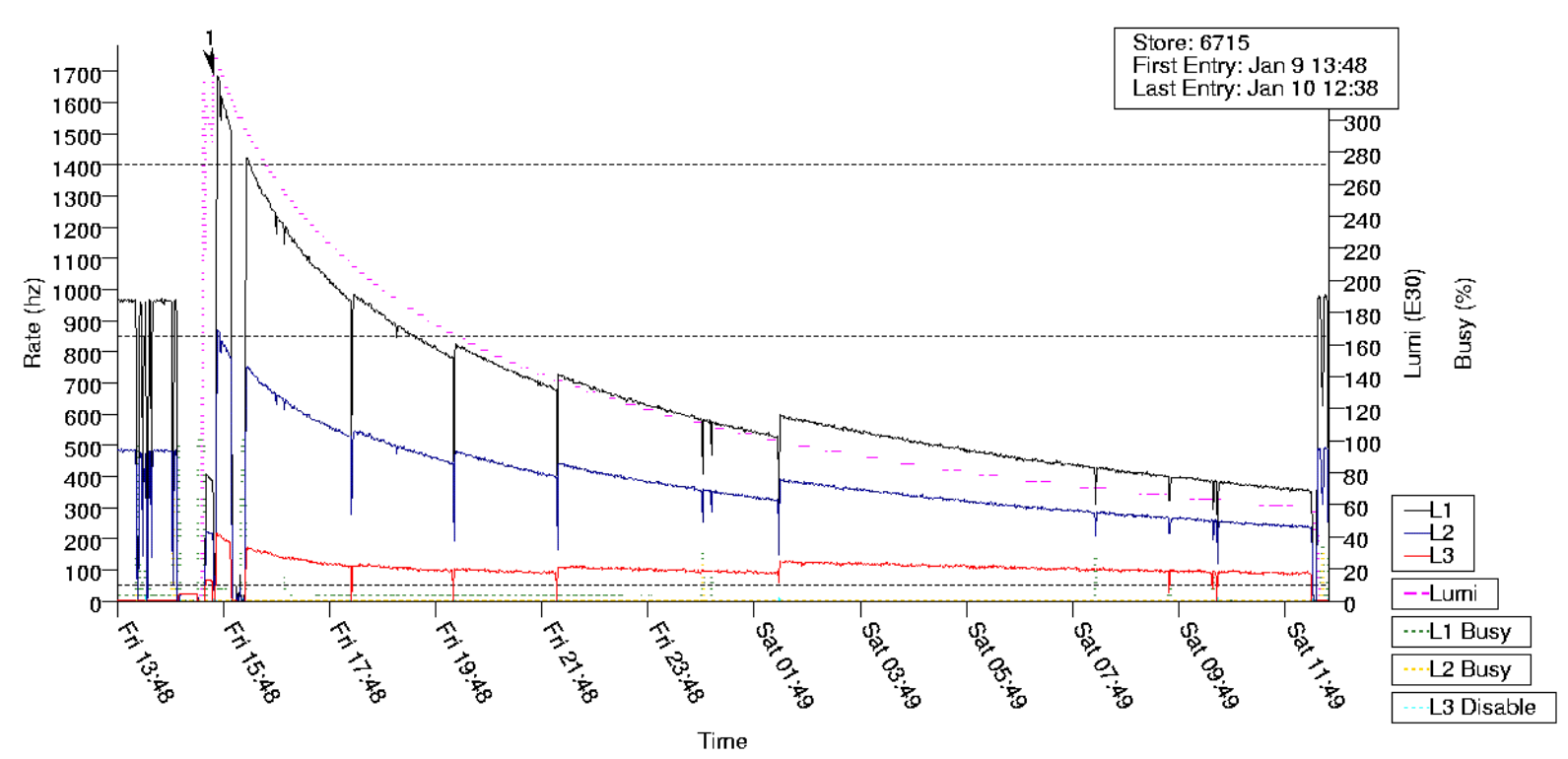

Figure 3.19 Example of trigger rates and luminosity at D $\varnothing$ taken from one round of Tevatron collisions [63]. 


\section{Object Identification}

The D $\varnothing$ detector records raw data as ones and zeroes, which are stored on hard drives and correspond to bits of electric signal collected when particles interact with the detector. The goal of object identification in $\mathrm{D} \emptyset$ is to reverse this process and put all of the signals back together into particles. This task is performed by a series of algorithms that reconstruct these signals into photons, electrons, muons, jets and the energy imbalance left by the passage of a neutrino.

\subsection{Track Identification}

Tracks are made up of the deposits of charge left behind as charged particles propagate through the layers of the tracker. Tracks are used for object identification, such as differentiating between photons and electrons, which look the same in the calorimeter, and for measuring momentum. Hits in the SMT and CFT are formed into clusters, which are fed into pattern recognition software to search for tracks.

The basic idea of the tracker is shown in Figure 4.1. When a charged particle passes through the tracker, it will interact with strips in the SMT and fibers in the CFT, resulting in an analog signal. After calibration, this signal is digitized into ADC (analog to digital converter) counts. Clusters are made of adjacent channels with ADC counts above a minimum level. The locations of hits input into the track finding algorithms are taken from the centers of the clusters. The resolution of the hits in the SMT is $\sim 10 \mu \mathrm{m}$ perpendicular to the direction of the strips (azimuthal) and $\sim 35 \mu \mathrm{m}$ in the $z$ direction along the strips. The resolution of these values in the CFT is larger: $100 \mu \mathrm{m}$ in the azimuthal direction and $2 \mathrm{~cm}$ in the $z$ direction. 


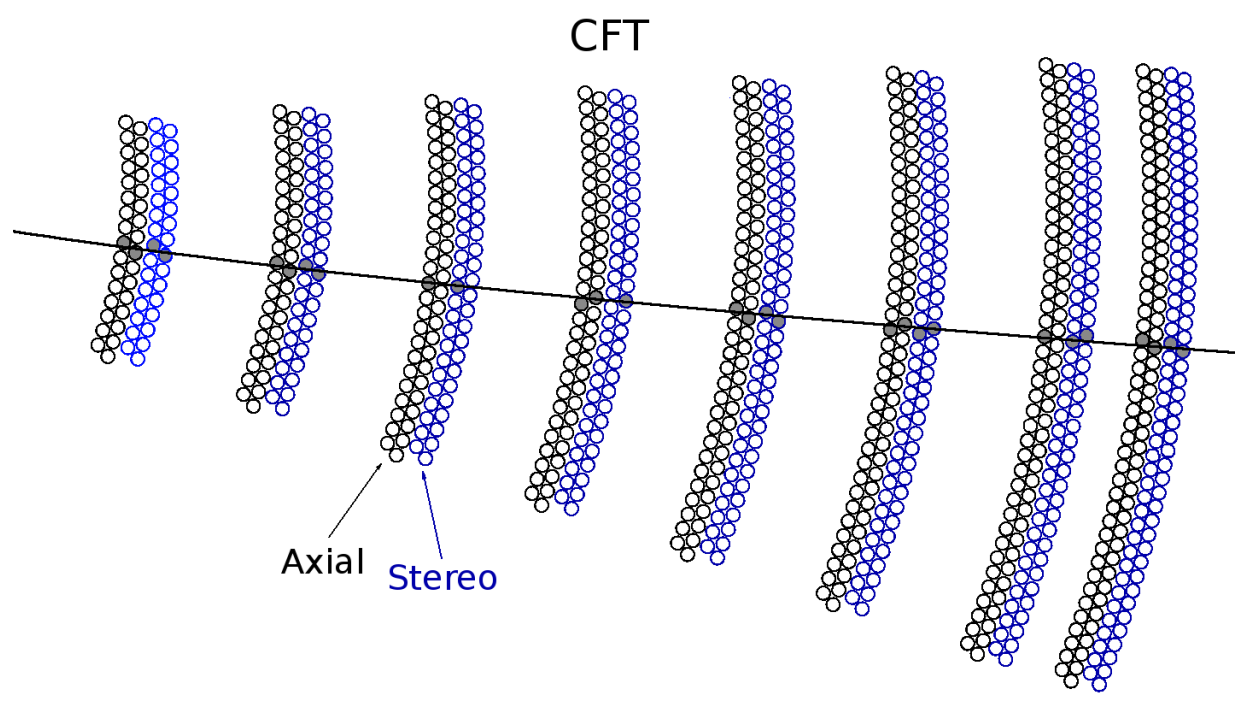

Figure 4.1 An example of a track from a charge particle passing through the CFT detector, reconstructed from clusters of hits.

Two different track finding methods, the Alternative Algorithm (AA) [64] and the Histogram Track Finder (HTF) [65], are used by DØ to ensure that all of the tracks in each event are found. Tracks of charged particles moving in a homogeneous magnetic field in a vacuum are characterized by three numbers: the radius of curvature, $\rho$, the distance of closest approach to the origin, $d_{0}$, and the direction of the track at the point of closest approach, $\phi$.

The AA track finding method starts with a hit in any layer of the SMT barrels or F-disks and works outward. The second point can be in any following layer, and the two points must have $\delta \phi<0.08$. The third point is in any layer after that, and must have a minimum radius of $30 \mathrm{~cm}$, which corresponds to $p_{T}>180$ $\mathrm{MeV}$ and must have $d_{0}<2.5 \mathrm{~cm}$ and $\chi^{2}<16$ for the track candidate. All candidates are passed to a Kalman filter [66], which uses these candidates to find tracks throughout the entire tracking system. The Kalman filter contains a detailed map of the magnetic field and the material in the detector [67], and uses this information to extrapolate the trajectories of the tracks to the next layers of the tracking system. Any new hits found within the extrapolated trajectories are included in the tracks. Track candidates are not allowed to have more than two consecutive missed layers of hits and cannot share more than $2 / 3$ of their hits with other track candidates. The hits for a given track must have $\chi^{2}<16$. 
The HTF technique takes the all of the hit positions in $x \times y$ space and applies a Hough transformation [68] to convert the hit positions in the SMT into lines in $\rho \times \phi$ space. These lines fill a 2D histogram and the track candidates correspond to peaks in the histogram where the lines cross, with each candidate having the parameters $\rho$ and $\phi$ of the peak. A Kalman filter is applied to these track candidates and removes tracks with large $\chi^{2}$. Another Kalman filter extrapolates the tracks in the CFT. A reverse of this method is also used, starting with hits in the CFT and extrapolating the track candidates back into the SMT.

\subsection{Primary Vertex Reconstruction}

The point where a $p \bar{p}$ collision occurs is known as a vertex. The average number collisions per crossing is about two, but can be higher, especially during portions of the run with higher luminosities. The goal of primary vertex construction is to distinguish an interesting physics event, such as $t \bar{t}$ production, that caused the trigger to fire from the other collisions during the same bunch crossing, known as the underlying event or minimum bias interactions. The point along the beam pipe where an interesting physics event originated is known as the primary vertex. Precisely locating the primary vertex is useful for rejecting backgrounds, such as particles from the underlying event and cosmic rays.

Tracks used to find the primary vertex must have $p_{T}>0.5 \mathrm{GeV}$ and two or more hits in the SMT if the tracks are within the geometry of the SMT [69]. Tracks within $2 \mathrm{~cm}$ of each other are clustered together using a clustering algorithm. Vertex fits are performed for each cluster in a two pass process. In the first pass, a fit finds the best location of the common vertex. If the $\chi^{2}$ per number of degrees of freedom $\left(\chi^{2} / n d f\right)$ for the vertex is greater than 10, the track with the largest contribution to the vertex is removed. This procedure is repeated until the fit reaches $\chi^{2} / n d f<10$. In the second pass, only tracks that are within 5 standard deviations of the beam spot calculated in the previous pass are included. A similar fitting technique is used, removing tracks until the fit reaches $\chi^{2} / n d f<10$.

To figure out which of these vertices is the primary vertex, the transverse momenta of the tracks at each vertex are used [70]. Tracks from vertices originating from the underlying event tend of have lower $p_{T}$. Using this information, a prob- 
ability for each vertex to come from a minimum-bias collision is created. The vertex with the lowest minimum-bias probability is the primary vertex.

\subsection{Electrons}

Electrons shower in the calorimeter, leaving clusters of energy in the EM calorimeters and tracks in the tracker. Photons, which are not used in this analysis, have the same calorimeter signature, but do not leave a track in the central tracking system. There are two major background sources that mimic electron decay: $\gamma \rightarrow e^{+} e^{-}$and jets with $\pi^{0} \rightarrow \gamma \gamma[71]$. Smaller background sources include charged pions decaying to electrons and fluctuations in the shape of hadronic showers.

Electron reconstruction starts with information from the calorimeter. An electromagnetic $(\mathrm{EM})$ tower is defined by adding the energies measured in a section of $0.1 \times 0.1$ in $\Delta \eta \times \Delta \phi$ occupying all four layers of the EM calorimeter plus the first hadronic layer. Using a simple cone algorithm centered on an EM tower with the highest transverse energy $\left(E_{T}=E / \cosh (\eta)\right)$, all energies above $E_{T}>50 \mathrm{MeV}$ from towers in a radius of $R=\sqrt{\Delta \eta^{2}+\Delta \phi^{2}}$ from the central tower are added together, forming an EM cluster.

A set of basic selection criteria are imposed on the the EM clusters to ensure they come from electron or photon-initiated showers:

- The minimum $E_{T}$ in a cluster must be $1.5 \mathrm{GeV}$.

- The cluster must be narrow, having greater than $40 \%$ of the energy contained in central tower.

- At least $90 \%$ of the cluster energy must be deposited in the electromagnetic layers. This criterion is known as the EM fraction,

$$
f_{E M}=\frac{E_{E M}}{E_{t o t}}>0.9
$$

where $E_{E M}$ is the energy in the EM layers and $E_{t o t}$ is energy of all of the layers within the cone. 
- The cluster must be isolated in $\eta \times \phi$, with an isolation fraction, $f_{\text {iso }}<0.2$. The isolation fraction is defined as

$$
f_{\text {iso }}=\frac{E_{t o t}(R<0.4)-E_{E M}(R<0.2)}{E_{E M}(R<0.2)}<0.2,
$$

where $E_{\text {tot }}(R<0.4)$ is the summed energy of all the towers except for those in the coarse hadronic calorimeter within a cone of radius of $R=0.4$ from the central tower, and $E_{E M}(R<0.2)$ is the summed energy in the EM layers in a cone of radius $R=0.2$.

Clusters passing the above criteria are considered as electron and photon candidates. The centroid of the cluster is found by using the cell energies in the finely segmented third layer of the EM calorimeter. The primary vertex, the shower centroid and information from the preshower detectors are all combined to calculate the 4-momentum of the particle. To be considered an electron, track with $p_{T}>1.5$ $\mathrm{GeV}$ must be found within a $0.05 \times 0.05$ in $\Delta \eta \times \Delta \phi$ "road" pointing leading back to the primary vertex. Electrons require further isolation, with $f_{\text {iso }}<0.15$.

Another criteria is the shower shape from electrons, which differs from the shape of hadronic showers. The H-Matrix technique uses a $7 \times 7$ covariance matrix that takes into account the shape of the shower, as well as the correlations between energy deposits in different calorimeter cells. Seven inputs are included in the covariance matrix:

- The fraction of the energy in each of the four EM layers of the calorimeter, which reflects the longitudinal shower development.

- The cluster width in the $\mathrm{r}-\phi$ plane of the third layer of the EM calorimeter, which reflects the lateral shower development.

- The logarithm of the total energy of the shower and the longitudinal $(z)$ position of the primary vertex, to include energy and impact parameter dependence.

A sample of $N$ simulated electrons is used to determine the expected shower 
shape and construct the covariance matrix

$$
M_{i j}=\frac{1}{N} \sum_{n=1}^{N}\left(x_{i}^{n}-\bar{x}_{i}\right)\left(x_{j}^{n}-\bar{x}_{j}\right)
$$

where $x_{i}^{n}$ is the value of the $i$ th variable for the $n$th simulated electron and $\bar{x}_{i}$ is the mean value for the $i$ variable. The H-matrix is the inverse of the covariance matrix, $H=M^{-1}$. For each electron candidate, a $\chi^{2}$ test is constructed from the H-matrix:

$$
\chi_{\mathrm{H}-\text { matrix }}^{2}=\sum_{i=1}^{7} \sum_{j=1}^{7}\left(x_{i}-\bar{x}_{i}\right) H_{i j}\left(x_{j}-\bar{x}_{j}\right),
$$

where $x_{i}$ is the value of the $i$ th variable for the electron candidate. Since the simulated shower shape depends on $|\eta|$, different H-matrices are constructed for different $|\eta|$ ranges. This analysis requires a relatively small value of $\chi_{\mathrm{H} \text {-matrix }}^{2}<$ 50 .

Tracks in the road to the calorimeter from the primary vertex are tested for the spatial match between the track and the EM layer 3 of the cluster in $\phi$ and $z$, as well as the energy-to-momentum fraction, $E / p$, for the energy in the calorimeter, $E$, and the momentum of the track, $p$ :

$$
\chi_{E M-\text { spatial }}^{2}=\left(\frac{\delta \phi}{\sigma_{\phi}}\right)^{2}+\left(\frac{\delta z}{\sigma_{z}}\right)^{2}+\left(\frac{E_{T} / p_{T}-1}{\sigma_{E_{T} / p_{T}}}\right)^{2}
$$

where $\sigma_{x}$ is one standard deviation for resolution from variable $x$. If multiple tracks are in the road, the track with the lowest $\chi^{2}$ is used. The probability $P\left(\chi_{E M-s p a t i a l}^{2}\right)$ must be larger than zero. The $\chi^{2}$ using the solely the spatial differences between the extrapolated track and the cluster is referred to as

$$
\chi_{\text {spatial }}^{2}=\left(\frac{\delta \phi}{\sigma_{\phi}}\right)^{2}+\left(\frac{\delta z}{\sigma_{z}}\right)^{2} .
$$

Electron candidates passing all of the requirements listed so far are referred to as "loosely isolated" or "loose" (Top_loose) electrons. Electrons which are considered to be "tightly isolated" or "tight" (Top_tight) electrons also have an 
electron likelihood output of $L_{7}>0.85$. The electron likelihood has seven different input variables:

- Probability of a spatial track match, $P\left(\chi_{\text {spatial }}^{2}\right)$.

- $E_{T} / p_{T}$, the ratio of the calorimeter energy to the track momentum.

- $f_{E M}$, the fraction of the energy in the EM calorimeter.

- $\chi_{\mathrm{H}-\text { matrix }}^{2}$.

- The distance of closest approach (DCA) in $z$ between the track and the primary vertex

- The number tracks within a cone of $\Delta R=0.05$ around and including the track. Electrons should only have one track, while backgrounds such as $\pi^{0}$ decays may have more than one track.

- The sum of the transverse momenta of all the tracks inside a cone of $\Delta R=$ 0.4 around the track, excluding the track candidate. Additional tracks with large $p_{T}$ are more likely to come from jets.

Probability distributions for each of the input variables are produced from data for real (signal) and fake (background) electrons. The product of the probability of each input variable, $x_{i}$, gives the overall probability, $P(\vec{x})$, for the electron candidate:

$$
P(\vec{x})=\prod_{i} P\left(x_{i}\right)
$$

A likelihood is constructed from the overall probability of an electron candidate to be from signal or background:

$$
\mathcal{L}(\vec{x})=\frac{P_{\text {sig }}(\vec{x})}{P_{\text {sig }}(\vec{x})+P_{b k g}(\vec{x})} .
$$

Electron candidates which are more signal-like have $\mathcal{L}(\vec{x})$ closer to one, while candidates that are more background-like tend toward zero. 


\subsection{Muons}

Muons are identified and measured with the tracking system, the calorimeters and the muon detectors $[72,73]$. The three layers of the muon detector system provide positive muon identification and, if a track segment can be reconstructed from the hits in the layers, a momentum measurement. Muon candidates reconstructed solely from this detector are known as "local" muons. Information provided by the central tracking system allows for precision measurement of muon momenta. Local muons that are matched with a central track are known as "central trackmatched muons." The calorimeter can independently confirm muon identification by the MIP signature left by a muon. This capability is known as "Muon Tracking in the Calorimeter," or "MTC." However, with an efficiency of only about 50\%, this procedure is not used for this analysis.

Different types of muons are defined by the number of hits in the three layers of the muon system and the quality of the matched track in the central tracking system. All of the types of muon identification requirements used at D $\varnothing$ are listed in References [73]. In the case of this analysis, muons are defined with following requirements:

- The "local" muon must have hits in all three (A, B and C) layers of the muon system. This requirement is know as the $n_{\text {seg }}=3$ requirement.

- The quality of the local muon must be version 2 of the "medium" definition, meaning:

- at least two A layer wire hits,

- one or more A layer scintillator hits,

- at least two wire hits in the combined BC layer,

- and at least one scintillator hit in the BC layer. For the central region, this requirement can be dropped.

- To veto cosmic muons, the time of flight between the collision and hits in the layers must be less than $10 \mathrm{~ns}$.

- The muon must be matched to a central track. 
- The matched central track must have version 2 of "trackmedium" quality. This definition means that track must have $|d c a|<0.04$ from the primary vertex if there is at least one SMT hit. If no SMT hits are found, the track must have $|d c a|<0.2$. The track fit must have $\chi^{2} / n d f<4$.

- To ensure that the muon does not arise from the decay of a heavy hadron, the central track of the muon must have $\delta R>0.5$ in $\eta \times \phi$ space from any good, non-vertex confirmed jets with $p_{T}>15 \mathrm{GeV}$ (more details in Section 4.5). This requirement is known as "deltaR" isolation.

Muons that fulfill the above criteria are known as "loosely isolated" or simply "loose" muons. Muons passing an additional isolation requirement known "TopP14" isolation are known as "tightly isolated" or "tight" muons. The TopP14 requirement means that:

- $\sum_{\# \text { of cells }} E_{T} / p_{T}^{\mu}<0.08$, where $\sum_{\# \text { of cells }} E_{T}$ is the sum of the transverse energies in all of the calorimeter clusters within a hollow cone of $0.1<$ $\Delta R<0.4$ centered around the track of the muon. Only clusters from the fine hadronic and electromagnetic calorimeters are considered.

- $\sum_{\# \text { of tracks }} p_{T} / p_{T}^{\mu}<0.06$, where $\sum_{\# \text { of tracks }} p_{T}$ is the sum of the transverse momenta of every track within a cone of $\Delta R<0.5$ around the muon track and with $\Delta\left(z_{0}^{\text {track }}, z_{0}^{\text {muon }}\right)<2 \mathrm{~cm}$. The transverse momentum from the track of the muon in consideration is, perhaps obviously, not included in the sum.

\subsection{Jets}

Jets are collimated showers of particles from the decay of a hadron originating from quarks or gluons produced at the primary vertex. In the hadron rest frame, the decays are more-or-less spherical, but the large boosts from top quarks $\left(m_{t}=172.9\right.$ $\mathrm{GeV})$ and $W$ bosons $\left(m_{W}=80.4 \mathrm{GeV}\right)$ decaying to lighter particles $\left(m_{\text {light }} \leq 5\right.$ $\mathrm{GeV}$ ) cause these decays to become cone-shaped. As the decay of top quarks to the lepton+jets channel creates at least four jets, the jet finding algorithms are an important base for the analysis. 


\subsubsection{Jet Reconstruction}

Jets at $\mathrm{D} \varnothing$ are reconstructed using the Run II cone algorithm $[74,75]$. Reconstruction starts with the calorimeter cells. Each cell is considered to have zero mass and is assigned a 4 -vector, $\mathbf{p}^{\text {cell }}=\left(E^{\text {cell }}, \vec{p}^{\text {eell }}\right)$, where $E^{\text {cell }}$ is the energy measured in the cell and $p^{\text {rell }}$ is momentum vector of the cell, with a magnitude of $E^{\text {cell }}$ and a direction defined by the primary vertex. A reconstructed tower is built from each $0.1 \times 0.1$ in $\eta \times \phi$ geometrical tower that contains a cell above the noise thresholds. The 4-momentum of the tower, $\mathbf{p}^{\text {tower }}$, is found using the "E-scheme":

$$
\mathbf{p}^{\text {tower }}=\sum_{i} \mathbf{p}_{i}^{\text {cell }} .
$$

The towers are input into a $p_{T}$-ordered list. The Simple Cone Algorithm takes this list and builds "preclusters," which are seeds for the Run II Cone Algorithm. If a tower has $p_{T}^{\text {tower }}>500 \mathrm{MeV}$, it can initiate a precluster. Any other towers within $\Delta R<0.3$ from the initiating tower and with $p_{T}>1 \mathrm{MeV}$ are combined with the precluster and removed from the list of towers, along with the initiating tower. After the list of towers has no more towers with $p_{T}^{\text {tower }}>500$ $\mathrm{MeV}$, preclustering is complete and only preclusters that contain more than one tower and have $p_{T}^{\text {precluster }}>1 \mathrm{GeV}$ are kept.

Once preclustering is complete, the $p_{T}$-ordered preclusters are used as seeds for creating "proto-jets." For each precluster, the distance $\Delta R=\sqrt{(\Delta y)^{2}+(\Delta \phi)^{2}}$ is calculated between the precluster and the nearest proto-jet. If $\Delta R$ is less than $R_{\text {cone }} / 2$, where $R_{\text {cone }}$ is 0.5 for this analysis, then the precluster is added to the proto-jet and the algortihm goes to the next precluster in the list. If not, the precluster initiates a new proto-jet candidate. For each proto-jet candidate, the 4-momenta of the towers within the cone are summed to form the 4-momentum of the proto-jet, $\mathbf{p}^{\text {proto-jet }}$. A cone is centered around the new proto-jet candidate and the procedure is repeated. This repetition takes place until a stable proto-jet candidate is found. If $p_{T}^{\text {proto-jet }}<4 \mathrm{GeV}$, the proto-jet is not kept. Preclusters with $\Delta R<0.25$ from the proto-jet are removed from the list of preclusters, and the entire process repeats to find more proto-jets.

An additional step is applied to the proto-jets to check if any proto-jets overlap 
each other and ensure that energy is not double counted. The proto-jets are grouped into a $p_{T}$-ordered list. If a proto-jet shares any preclusters with any other proto-jet, the sum of the $p_{T}$ of the shared preclusters is calculated. If the sum has more than $50 \%$ of $p_{T}$ of the neighboring proto-jet, the two proto-jets are merged. If the fraction is smaller than $50 \%$, the preclusters are split between the two protojets, according to which proto-jet is closest in $\Delta R$. After "Merging/Splitting" has taken place, these are the jets that are used by the analysis. Jets are required to have $p_{T}>8 \mathrm{GeV}$.

Because these jets still might contain "fake" jets created mainly by noise from the calorimeter, further quality requirements are used [76]:

- The transverse energy of the most energetic cell must be less than ten times larger than the transverse energy of the second most energetic cell.

- No tower in the jet can contain more than $90 \%$ of the jet energy.

- The fraction of the energy in the EM calorimeter must greater than 5\%. In addition, it must be less than $95 \%$ to different jets from EM objects.

- The fraction of energy in the course hadronic calorimeter must be less than $46 \%$ in the central calorimeter and $33 \%$ in the end calorimeter.

- Each jet must be confirmed by the Level 1 trigger. This confirmation means that the ratio of the energy read out from a $0.2 \times 0.2$ in $\eta \times \phi$ trigger tower to the energy of the jet,

$$
L 1_{\text {ratio }}=\frac{p_{T}^{\text {trigger }}}{p_{T}^{\text {jet }}\left(1-f_{C H}\right)},
$$

must be greater than 0.5 . The quantity $p_{T}^{\text {trigger }}$ is the scalar sum of the transverse mometna from the $100 \mathrm{~L} 1$ towers with the largest energy inside a cone of radius $\Delta R=0.5$ around the jet. Energy from the coarse hadronic calorimeter is not included in the jet.

In Run IIb, jets must pass an the additional requirement, known as vertex confirmation, and have at least two tracks within the cone of the jet that are associated with the primary vertex. 


\subsubsection{Jet Energy Scale}

The jet energy measured by the detector is not the same as the energy of the particles that decayed to the jet, due to imperfect detector response, as shown in Figure 4.2. The purpose of jet energy scale (JES) corrections is to correct the measured energies of the jets back to the level of jets of stable particles before interaction with the detector to get a more accurate energy measurement. To measure this correction, the same cone algorithm that is used for the detector is applied to "particle jets," that is jets of stable particles. The energy of detected jets $\left(E_{\text {jet }}^{\text {raw }}\right)$ is related to the energy of particle jets $\left(E_{\text {jet }}^{\text {ptcl }}\right)$ by the following equation:

$$
E_{j e t}^{p t c l}=\frac{E_{j e t}^{r a w}-O}{F_{\eta} \cdot R \cdot S} \cdot k_{b i a s}
$$

where:

- $O$ is the offset energy correction resulting from noise from electronics and uranium decays, multiple $p \bar{p}$ collisions and previous bunch crossings (pileup),

- $R$ is the absolute response, determined by balancing $\gamma+$ jet events in transverse energy (photons have better resolution),

- $F_{\eta}$ is the relative response, and takes into account changes in response for different jet pseudorapidities,

- $S$ is the shower correction, which takes into account energy deposited to and from the cone due to the magnetic field and other jets,

- and $k_{b i a s}$ is the correction for remaining biases from zero suppression effects.

The average values for the quantities above are measured, leading to the corrected jet energy,

$$
E_{j e t}^{c o r r}=\frac{E_{j e t}^{r a w}-\hat{O}}{\hat{F}_{\eta} \cdot \hat{R} \cdot \hat{S}} \cdot \hat{k}_{b i a s}
$$

where $\hat{x}$ is the average value that is measured for variable $x$. 


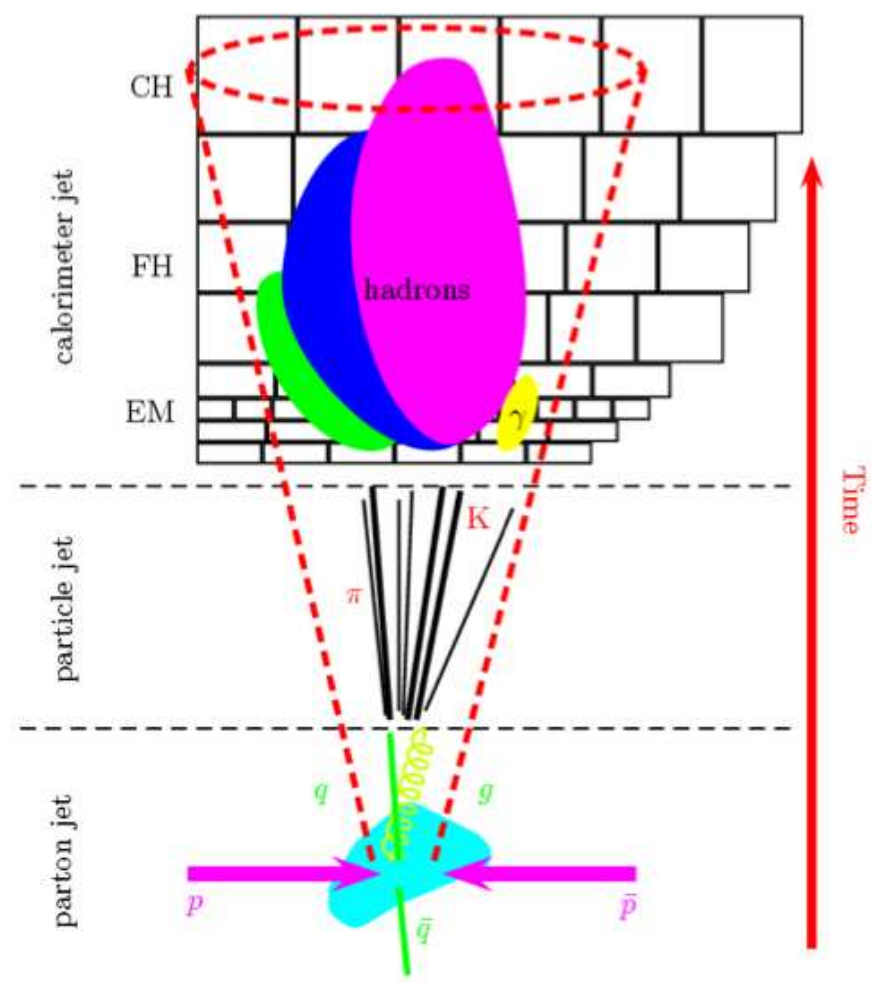

Figure 4.2 Diagram of a hard-scattered parton decaying into a jet. [77]

The offset correction, $\hat{O}$, is measured from the energy in the calorimeter from "minimum-bias" and "zero-bias" events. Minimum-bias events are triggered by the luminosity monitor, meaning that some sort of $p \bar{p}$ scatter occurred. Zero-bias events are even less discerning, and are triggered by the Tevatron clock at every bunch crossing. The offset correction is anywhere from about $2 \mathrm{GeV}$ for $\eta_{j e t}=0$ to about $22 \mathrm{GeV}$ for $\eta_{j e t}=3.3$. For this analysis, the maximum offset correction is about $10 \mathrm{GeV}$.

The absolute response correction, $\hat{R}$, is measured with the MPF (Missing $E_{T}$ Projection Fraction) method from events with one photon and one jet, as shown in Figure 4.3. The photons in the events are selected with stringent criteria to be extremely pure. The jet and the photon are required to be back-to-back, with $\Delta \phi(\gamma$, jet $)>3.0$ radians. To estimate the particle level jet energy, the response is measured as a function of $E^{\prime}=p_{T}^{\gamma} \cosh \left(\eta_{\mathrm{jet}}\right)$. Using this variable helps to minimize detector effects. The response is calculated by measuring the imbalance in the transverse momentum. If the response is 1 , no imbalance will be present. 
Results from data from the response correction are shown in Figure 4.4.

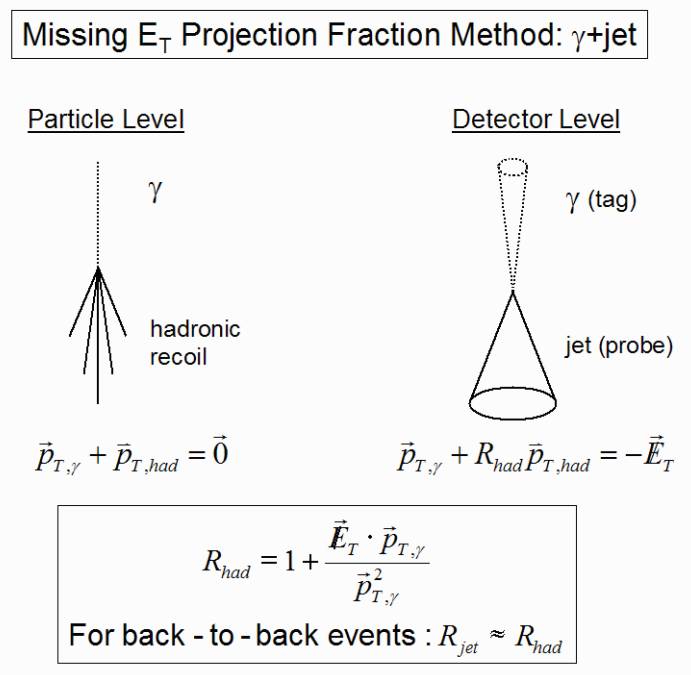

Figure 4.3 Diagram describing the MPF method. [77]

The $\eta$ dependent response, $\hat{F}_{\eta}$, is used because of differences in response between the ICD and the central and end calorimeters. The main purpose of relative response is to calibrate forward jets with respect to central jets.

The showering correction, $\hat{S}$, is applied to take into account energy leaking out of or coming into the jet from the movement of particles out of or into the cone. This correction takes into account detector effects, but not physical effects such as large-angle gluon radiation. The correction is determined by measuring the amount of energy in cones of various radii in the jet, the so called shower profile. Templates are made from MC simulation of the shower profile for particles in and outside of the jet. These templates are fit to shower profiles measured from $\gamma+$ jet data to find the size of this correction.

The remaining bias correction, $\hat{k}_{\text {bias }}$, is due to the fact that the offset measured in zero and minimum bias events do not exactly correspond to the offset for a jet. Additionally bias comes from the compactness of photons versus jets used for the MPF method. The size of this correction is small, typically less than $2 \%$, leading to $k_{\text {bias }} \geq 0.98$.

The fractional uncertainty on the jet energy is shown in Figure 4.5. The uncertainty starts out large and then decreases as the energy increases. The main 


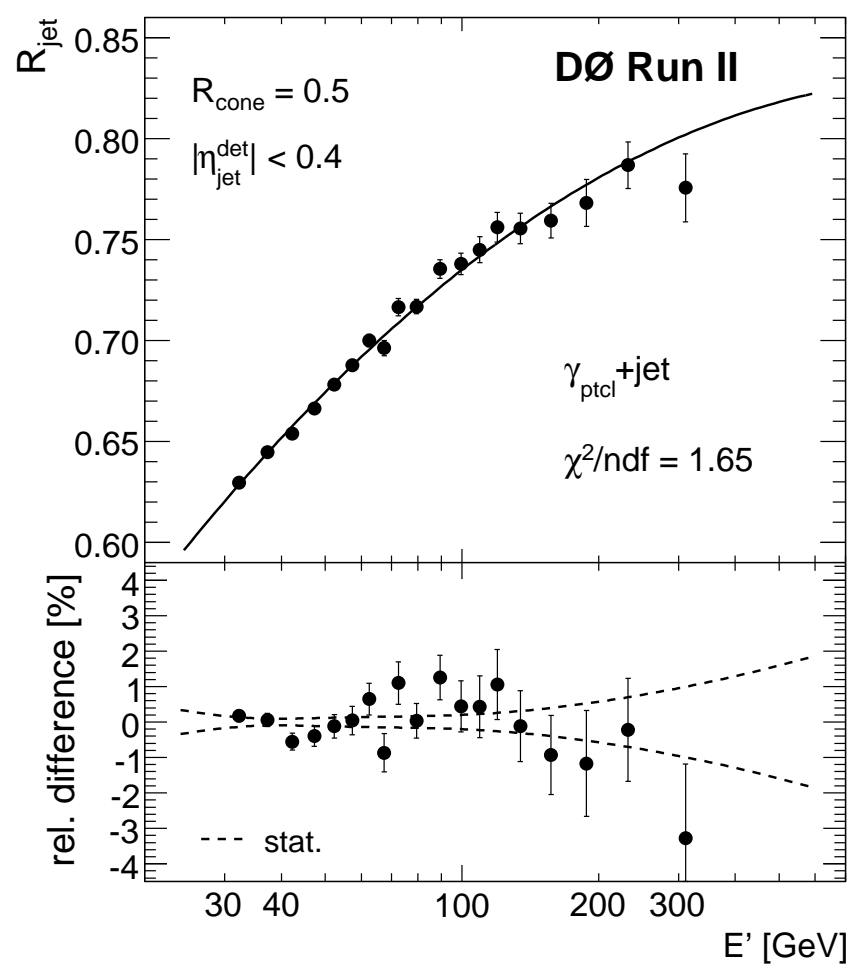

Figure 4.4 Plot of the absolute response for photon+jet events. [77]

sources of uncertainty are the uncertainty in the photon energy scale, coming from the uncertainty from the $Z \rightarrow$ ee peak and the electron-to-photon scale uncertainty. Contamination of the $\gamma+$ jet sample also plays a role, as well as limited statistics at very high energies.

\section{$4.6 \quad b$ Jets}

Each $t \bar{t}$ decay contains two jets originating from $b$ quarks. The background processes, particularly $W+$ jets, have a much smaller fraction of decays that contain $b$ quarks. For this reason, we use the ability of the detector, particularly the SMT, to differentiate between jets arising from $b$ quarks, and jets arising from gluons and lighter flavors of quarks. There are two properties that are specific to heavy flavor jets, which originate from hardons containing $c$ and $b$ quarks, that are helpful for identifying them in the detector: 


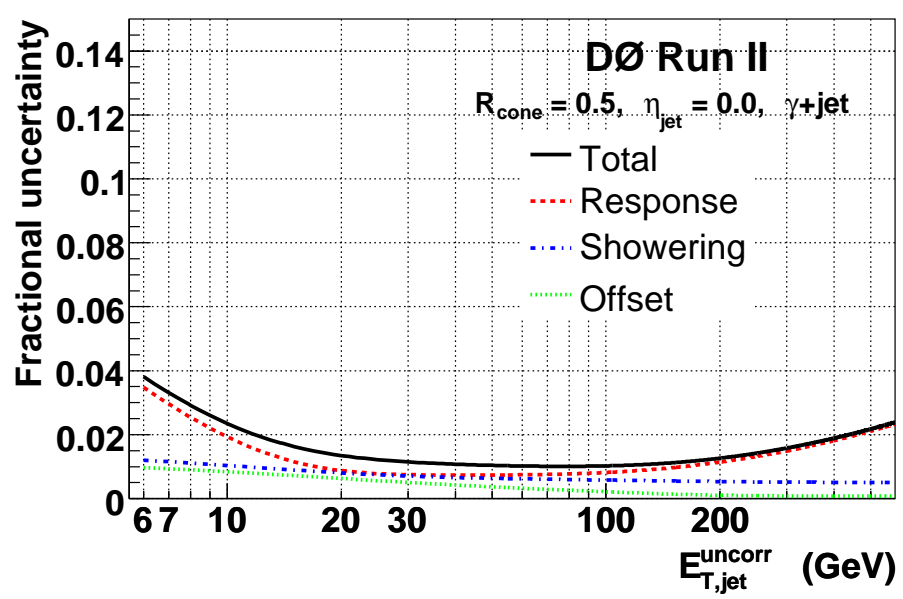

Figure 4.5 Plot of the fractional JES uncertainty. [77]

1 Heavy flavor hadrons have long lifetimes and are produced at nearly the speed of light, meaning their internal clocks experience time dilation and they travel resolvable distances from the primary vertex before decaying. For the case of hadrons containing $b$ quarks, the average decay length is $3 \mathrm{~mm}(c \tau \gamma=$ $\left.0.491 \mathrm{~mm} \times \frac{30 \mathrm{GeV}}{5 \mathrm{GeV}}\right)$, and somewhat shorter for hadrons containing $c$ quarks, which have $c \tau=0.412 \mathrm{~mm}[16]$.

2 Hadrons containing $c$ and $b$ quarks have a sizable branching fraction of decays containing leptons, about $10 \%$ of all decay modes for each type of lepton [16]. Jets originating from lighter quarks have a much lower probability of containing leptons. If a low energy lepton is contained within the cone of a jet, then it has a high probability of being a heavy flavor jet.

Multiple ways for identifying $b$ jets, also known as $b$ tagging methods, have been developed at $\mathrm{D} \varnothing$ :

- The Counting Signed Impact Parameter (CSIP) method uses the significance of the impact parameter relative to the primary vertex of tracks inside the cones of jets [78]. To ensure that the tracks are inside the cone, this method uses the sign of the dot product of the direction of the impact parameter and the direction of the jet. A jet must have a minimum number of tracks with significant signed impact parameters to be tagged as a $b$ jet. 
- The Jet Lifetime Probability (JLIP) method calculates the probability that a jet originates from the primary vertex by using the impact parameters of the tracks in the SMT [79]. Jets with small probabilities are tagged as $b$ jets.

- The Secondary Vertex Tagger (SVT) method calculates displaced vertices from the decay of $b$ hadrons $[80,81,82]$. This method requires jets to have at least two tracks inside their cones. Using methods similar to the calculation of the primary vertex, the secondary vertices of the tracks inside the jets are calculated. If the magnitude of the significance (value/resolution) of the secondary vertex in $x$ and $y$ is greater than a specified value, the jet is $b$ tagged. Unlike the CSIP method, jets in the SVT method can have negative significances.

- The Soft Lepton Tagging (SLT) method tags jets that have low $p_{T}$ muon inside their cone [83]. This method is not included in this analysis, and is the only method which $b$ tags jets based on their decay mode, rather on their decay length.

- The Neural Net (NN) algorithm uses seven different variables taken from the CSIP, JLIP and SVT methods [84]. No information from the SLT method is included in the NN algorithm. The NN algorithm outputs a value between 0 and 1 for each jet. Larger neural net values indicate that the jet has a high probability of originating from a $b$ hadron. Figure 4.6 shows the neural net output for simulated $b \bar{b}$ events and light jets from simulated multijet events, as well as the efficiency and mis-tag rates for the NN algorithm and the stand alone JLIP method.

In this analysis, a jet must have an neural net value of $\mathrm{NN}>0.2$ to be tagged as a $b$ jet. Internally, this value is known as the "L4" working point. The tagging efficiency for $t \bar{t}$ events is approximately $70 \%$ and the mis-tagging is rate for $W+$ jets events is about $8 \%$.

To be considered for $b$ tagging, jets must pass the "taggability" requirement, which means having at least one track inside their cone. Jets in data are tagged directly from the NN output. Because the simulation of the tracking system is not perfect, the $b$ tagging probabilities in simulation are not used. Instead, the 

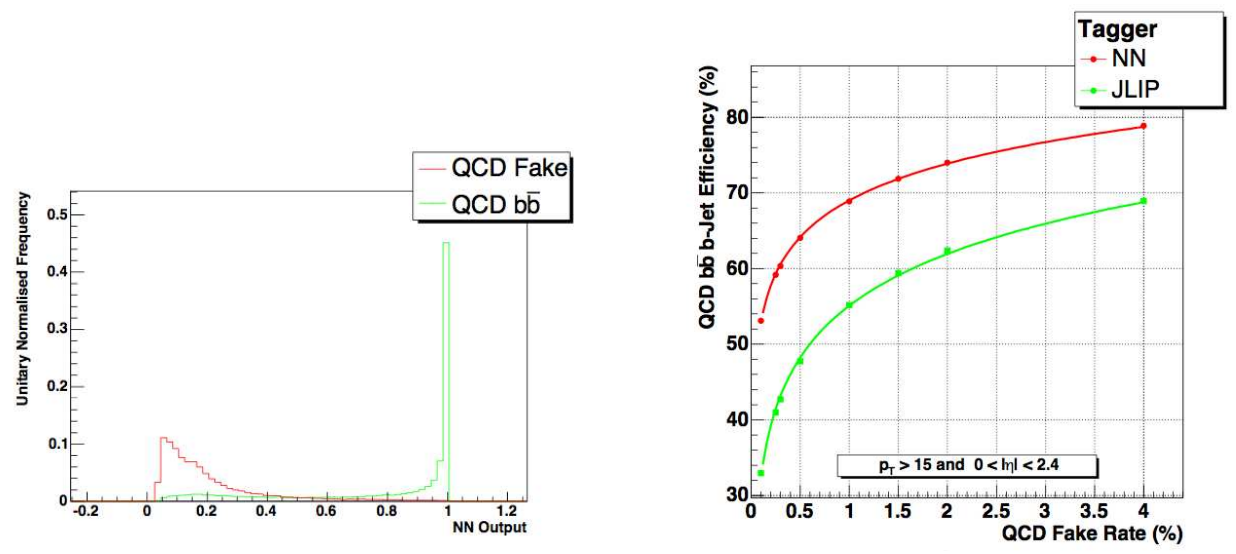

Figure 4.6 The neural net outputs for jets from simulated $b$ hadrons and light hadrons (left). Comparison of the efficiency and mis-tag rates for the NN algorithm and the JLIP method (right). [84]

probability of a jet to be $b$ tagged is calculated with a data-derived parameterization known as the Tag Rate Function (TRF). For each jet, the $p_{T}$ and $\eta$ values are put into the TRF to calculate the probability that the jet was $b$ tagged. This analysis applies the TRFs to simulation with method known as Direct Tagging, discussed further in Section 5.3.1.

\subsection{Missing Transverse Energy}

The overall momentum of the initial collision is zero, as the protons and antiprotons have equal masses and equal and opposite velocities. However, when protons and anti-protons collide, remnants from the proton and antiproton that did not take part in the collision travel down the beam pipe without interacting with the detector. For this reason, the $z$ momentum of the colliding partons (quarks or gluons) is unknown. However, momentum transverse to the beam pipe is very close to zero. Instead of the conservation of momentum, we use the conservation of transverse momentum. An imbalance in the total transverse momentum, known as missing transverse energy $\left(\mathscr{E}_{\mathrm{T}}\right)$, is a sign that one or more particles originating from the collision were not detected. In the case of $t \bar{t}$ decay, this indicates the presence of a neutrino.

Missing transverse energy starts with the calculation of the vector sum of 
energy deposits from calorimeter cells:

$$
\mathbb{E}_{\mathrm{T}}^{\text {uncorr }}=-\sum_{i} \bar{p}_{i}^{\text {cell }}
$$

where $\bar{p}_{i}^{\text {cell }}$ is the energy of $i$ th calorimeter cell with the direction of the coordinates of the cell in the EM and fine hadronic calorimeters. Cells from the coarse hadronic calorimeter are left out to reduce the noise.

Corrections are applied to $\mathbb{E}_{\mathrm{T}}{ }^{\text {uncorr }}$ to take into accounts muons, which leave only a fraction of their energy in the calorimeter, and reconstructed photons, electrons and jets, which have energy corrections that are more accurate than the sum of calorimeter cells included with these objects. Any calorimeter cell associated with a reconstructed object is removed the sum, leading to a corrected missing transverse energy of:

$$
\mathbb{E}_{\mathrm{T}}^{\text {corr }}=-\sum_{i} \vec{p}_{i}^{\text {cell }}-\sum_{\text {objects }}\left(\vec{p}^{\text {object }}-\sum_{i \in \text { object }} \vec{p}_{i}^{\text {cell }}\right),
$$

where all reconstructed objects are included and $\vec{p}^{\text {object }}$ is the momentum of the object after corrections, and $i$ is the index of the cells contained in each object. Only calorimeter cells that are not contained by any reconstructed object, including the projected path of the muon, are included in the computation of the fully corrected missing energy. 


\section{Data and Simulation}

This chapter describes the data sample and the computer-driven Monte Carlo event simulations used in this analysis.

\subsection{Data Sample}

The data sample used by this analysis was collected over multiple years, starting in April 2002 and ending in August 2009. Tens of thousands of runs are used in the analysis, numbering 151817 through 252918. There are two distinct periods of collection: Run IIa, which ended in February 2006 and collected the first $1.1 \mathrm{fb}^{-1}$ of data and Run IIb, which began in June 2006 and collected $4.3 \mathrm{fb}^{-1}$ of data. Different triggers were used in each run period. Details of the specific triggers used are listed in Tables 5.1 and 5.2 for Run IIa and in Table 5.3 for Run IIb. More information on the details of the $e+$ jets superOR, $\mu+$ jets superOR and single muon OR triggers can be found in Reference [85].

A total of 5.9 billion events were recorded by $\mathrm{D} \varnothing$ during these run periods. By including $e+$ jets and $\mu+$ jets specific triggers, the overall number of events input into the analysis is reduced by a factor of twenty to roughly 260 million, with 150 million events in the $e+$ jets channel and 110 million events triggered in the $\mu+$ jets channel.

The set of triggers in the $e+$ jets channel are made up of single electron triggers, which require energy in the EM calorimeter with or without a track, and the $e+$ jets triggers, which require two jets with $E_{T}>20 \mathrm{GeV}$, one jet with $E_{T}>25 \mathrm{GeV}$ and an electron with $E_{T}>15 \mathrm{GeV}$ that matches certain shower shape requirements. Similarly the triggers used in the $\mu+$ jets channel are made up of single muon 
triggers, which require hits in the muon system, with or without a loosely isolated track, and $\mu+$ jets triggers, which require at least one jet made with a simple cone algorithm with $E_{T}>35 \mathrm{GeV}$ and a muon with $p_{T}>3 \mathrm{GeV}$. Each selected event is required to pass one of these triggers.

\begin{tabular}{l|c|c} 
Triggerlist Version & Trigger & Integrated luminosity $\left[\mathrm{pb}^{-1}\right]$ \\
\hline \hline V8.0 - V14.99 & JT_125TT & 1078.81 \\
\hline T O T A L & & 1078.81 \\
\hline
\end{tabular}

Table 5.1 Integrated luminosity analyzed in $e+$ jets final states in Run IIa.

\begin{tabular}{l|c|c} 
Triggerlist Version & Trigger & Integrated luminosity $\left[\mathrm{pb}^{-1}\right]$ \\
\hline \hline V8.0 - V9.0 & MU_JT20_L2M0 & 24.80 \\
V9.0 - V10.0 & MU_JT20_L2M0 & 25.01 \\
V10.0 - V11.0 & MU_JT20_L2M0 & 10.70 \\
V11.0 - V12.0 & MU_JT20_L2M0 & 65.85 \\
V12.0 - V13.0 & MU_JT25_L2M0 & 231.84 \\
V13.0 - V13.2 & MUJ2_JT25 & 31.86 \\
V13.2 - V13.3 & MUJ2_JT25_LM3 & 16.11 \\
V13.3 - V14.0 & MUJ2_JT30_LM3 & 255.80 \\
V14.0 - V14.2 & MUJ1_JT25_LM3 & 0.01 \\
V14.2 - V14.3 & MUJ1_JT25_ILM3 & 21.89 \\
V14.3 - V15.0 & MUJ1_JT35_LM3 & 317.17 \\
\hline T O T A L & & 1001.02 \\
\hline
\end{tabular}

Table 5.2 Integrated luminosity analyzed in $\mu+$ jets final states in Run IIa.

\subsection{Event Simulation}

To get a handle on the effects of the detector on the signal asymmetry and predict the amount of asymmetry from background processes, this analysis needs event simulation. A set of computer programs known as Monte Carlo (MC) event generators simulate the physical processes using perturbative QCD and simulate how the decay products from these processes will interact with the detector. The term Monte Carlo is used because the programs rely on random numbers to sample the phase space of the interactions. The entire chain of event generation includes 


\begin{tabular}{l|c|c} 
Triggerlist Version & Trigger & Integrated luminosity[ $\left.\mathrm{pb}^{-1}\right]$ \\
\hline \hline V15.0 - V15.99 & JT125_L3J125 & 1619.77 \\
V16.0 - V16.99 & JT125_L3J125 & 2661.89 \\
\hline T O T A L & & 4281.66 \\
\hline
\end{tabular}

Table 5.3 Integrated luminosity analyzed in $e+$ jets and $\mu+$ jets final states in Run IIb.

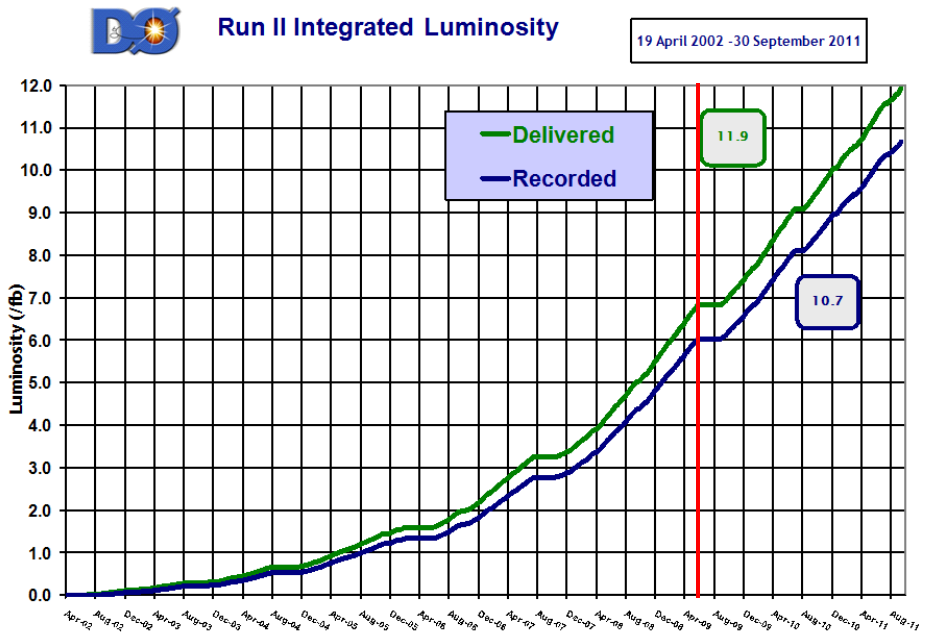

Figure 5.1 Integrated luminosity for Run II of DØ. This analysis uses data recorded up to the red line.

simulation of the hard scattering interaction, gluon radiation from the initial and final states, hadronization, the underlying event from minimum-bias interactions, effects of the detector and effects of reading out the detector from electronic signals.

Multiple event generators are used to calculate the matrix elements for $t \bar{t}$ production and background production, including MC@NLO [41, 42], ALPGEN [86], and PyтнiA [87]. The decay of unstable particles, known as parton showering and hadronization, is carried about by PYTHIA and HERWIG [88]. In hard scatter processes at hadron colliders, protons and antiprotons do not collide. Instead, partons from the proton and antiprotons interact with each other. In $t \bar{t}$ production these partons are usually a quark and an antiquark. The fraction of the momentum of the proton carried by the parton is referred to as $x$. Parton distribution functions (PDFs) are used to properly simulate the colliding momenta of the partons, as shown in Figure 5.2. This analysis uses the CTEQ6L1 PDF [89] 
with all event generators.

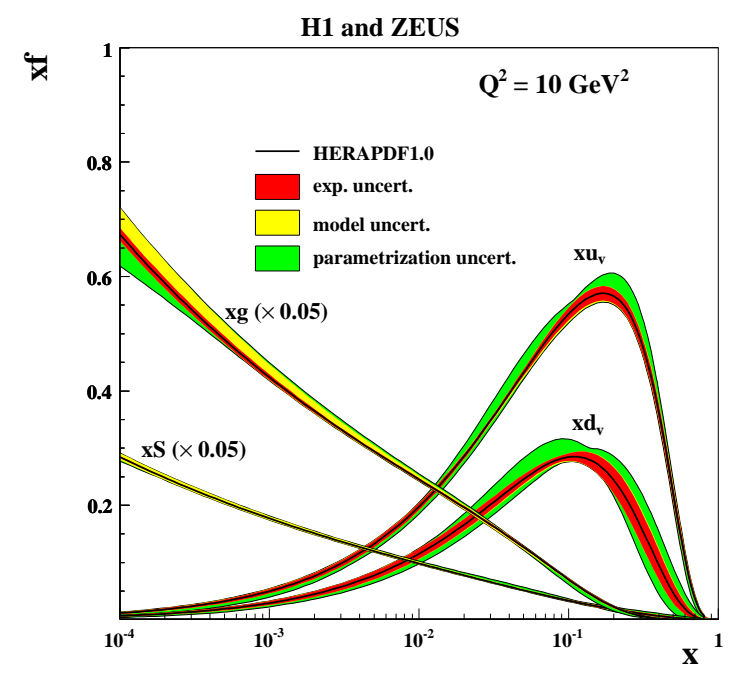

Figure 5.2 Parton distributions functions measured at HERA [90].

Particles produced by the event generators need to interact with the detector. The geometry and the material content are simulated with the DøGSTAR program [91], a simulation of the DØ detector based on GEANT [92]. GEANT is a software package that simulates the passage of elementary particles through matter. Physical processes for particles interacting with detector matter such as bremsstrahlung, pair production, ionization and hadronic interactions are included in GEANT. A program known as DøSIM simulates the digitization of the simulated data produced by DøGSTAR. To simulate the effects remnants of the proton and antiproton leftover from the hard scatter and collisions taking place outside of the hard scattering, DøSIM merges zero-bias events taken from actual collisions with the simulated events. The simulated events are reconstructed using the same algorithms that are used for events from data, described in Chapter 4.

The primary simulator of $t \bar{t}$ production is the MC@NLO event generator. To decay the $t$ and $\bar{t}$ quarks, MC@NLO utilizes the HERWIG event generator for parton showering and hadronization. The MC@NLO event generator includes processes at the order of $\alpha_{s}^{2}$ and at the order of $\alpha_{s}^{3}$. MC@NLO is designed to ensure that no double counting of gluon radiation occurs, so that processes such as $q \bar{q} \rightarrow t \bar{t} g$ and $q \bar{q} \rightarrow t \bar{t}$ (with a gluon radiated by the HERWIG parton shower) are distinguishable. 
Figure 5.3 contains plots of $A_{\mathrm{FB}}$ versus $y^{t}$ and the differential cross section for the transverse momentum of the top quark. Notice that there is no asymmetry for the HERWIG prediction, which only has leading order contributions, while both the MC@NLO and NLO predictions have similar, and somewhat large, asymmetries. For quantifying the systematic effects of the event simulation, $t \bar{t}$ signal is also produced with the ALPGEN event generator, with parton showering and hadronization carried out by PYTHIA using the MLM matching procedure [93]. Additional studies use $t \bar{t}$ events produced, showered and hadronized by the PYTHIA event generator.
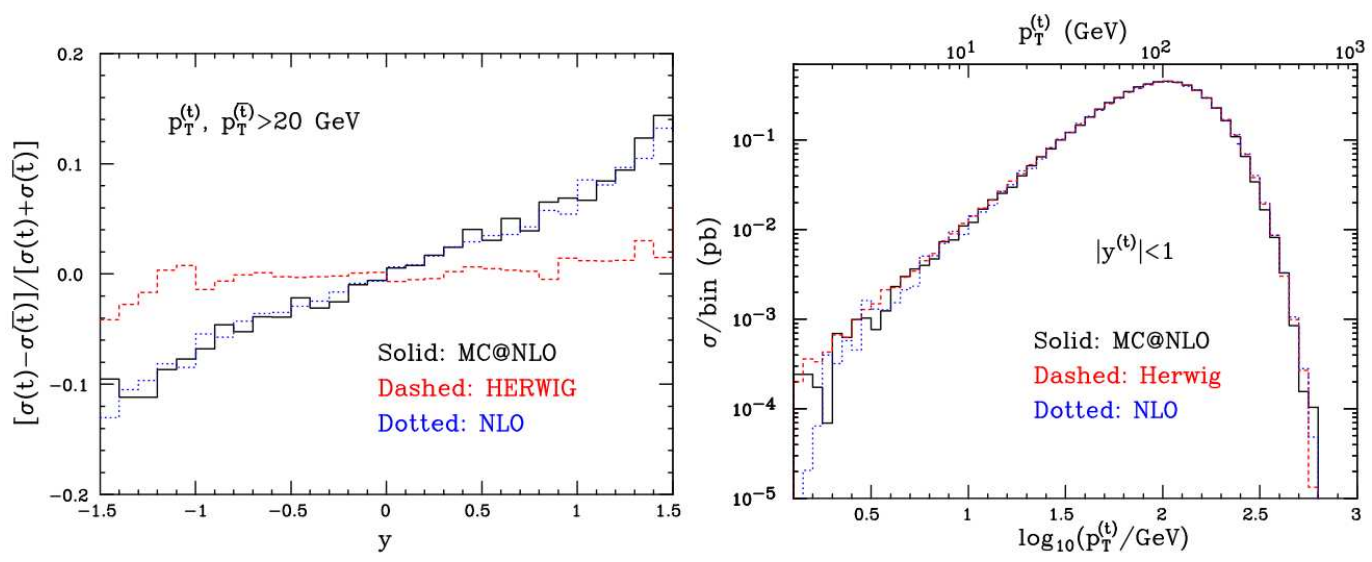

Figure 5.3 Production asymmetry versus top quark rapidity (left) and top quark tranverse momentum (right) from NLO predictions, HERWIG, and MC@NLO. HERWIGdoes not predict any asymmetry, while NLO predictions and MC@NLO do [42].

Events for the $W+$ jets background are simulated with the ALPGEN event generator, with the addition of PYTHIA for parton showering and hadronization. Three different $W+$ jets subsamples, $W b \bar{b}, W c \bar{c}$ and $W j j$, which includes the $W c j$ process, are included in the $W+$ jets subsample. For the most part the backgrounds are symmetric. In the case of $A_{\mathrm{FB}}^{l}$, the $W+$ jets background is highly asymmetric and is tested with background dominated samples. Other backgrounds are not included in the main portion of the analysis and are only used for testing:

Diboson $W W, W Z$ and $Z Z$ samples are generated with PYTHIA. 
Single top Single top events are simulated with the COMPHEP Monte Carlo generator [94].

$Z+$ jets The $Z+$ jets backgrounds are simulated with ALPGEN for events where the $Z$ boson decays to pair of electrons, muons or taus.

\subsection{Event Weights}

Rather than counting each event in simulation as one event, as is the case in data, events in simulation have weights to ensure that the relative frequencies between different types of events are correct and to more accurately match data. Real events from data are used for the zero-bias events included with the simulation. The instantaneous luminosities are higher in much of the data used in the measurement compared to when the zero-bias events were collected. For this reason, the luminosity profile of the zero-bias data used in the simulation is weighted to match the luminosity profile from the data used for the measurement.

In a similar fashion, the distribution of the $z$ coordinate of the primary vertex is Gaussian in simulation, but not in data [95]. The distribution of the $z$ coordinate of the primary vertex in simulation is weighted to match data. Because the trigger is not simulated, the probability that an event would pass one of the triggers is also included as weight. The fragmentation of $b$ hadrons is carried about by a variation of the Lund fragmentation model [96] known as Bowler functions [97]. This parameterization is included in PYTHIA, but the default settings do not agree with measurements from SLD [98], so a weight is included to account for this difference. The more complicated procedure of weighting simulated events by their probability of being $b$ tagged is described below.

\subsubsection{Direct $b$ Tagging}

The $b$-tagging probabilities in simulation are not correct, due to the fact that tracking efficiency in simulation is higher than in data. For this reason, dataderived $b$-tagging probabilities are applied to the simulation. The same $b$-tagging algorithm is applied on data and simulation, but flavor dependent scale factors are applied to each event. This method is known as direct tagging. Because all 
$b$-tagged jets are required to be "taggable" and contain at least one track, an additional taggability scale factor, derived from data, is applied to account for difference between the taggability efficiency in data and simulation. One way to include the taggability scale factor is to throw a random number for each jet in simulation. If the random number is less than the taggability scale factor, the jet is considered to be taggable. If the random number is larger, the jet is not considered to be taggable. The taggability scale factor is dependent on the jet $\eta$, the jet energy and the sign of the $z$ coordinate of the primary vertex.

In this analysis, we use a slightly different method. The overall probability for the jets in the event to be taggable is:

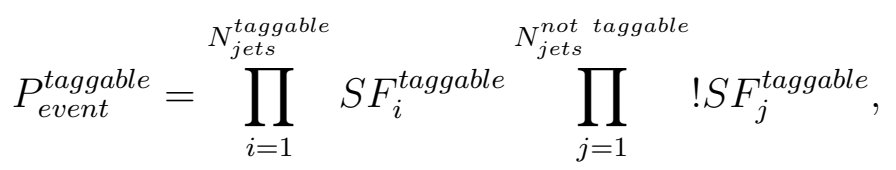

where $S F^{\text {taggable }}$ is the taggability scale factor and $! S F^{\text {taggable }}$ is $\frac{1.0-P_{\text {jet }}^{\text {taggable }}}{1.0-P_{\text {jet }}^{\text {tagable }} / S F^{\text {taggable }}}$. The quantity $P_{\text {jet }}^{\text {taggable }}$ is the probability for a jet to be taggable, derived from data.

Each event in simulation gets an additional weight built from the scale factors for each jet:

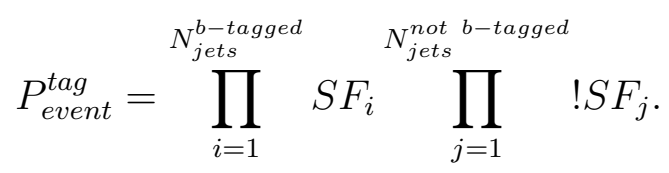

The scale factor $S F$ and the not-b-tagged scale factor $! S F$ are determined based on the value of $b$-tagging neural net output for the simulated jet:

$$
S F=\frac{\left(T R F_{\text {Data }}(a)-T R F_{\text {Data }}(b)\right)}{\left(T R F_{M C}(a)-T R F_{M C}(b)\right)},
$$

where $a$ and $b$ are the tighter $(b)$ and looser $(a) b$-tag working points that the NN output of the jet lies between. $T R F_{\text {Data }}$ and $T R F_{M C}$ are the Tag Rate Functions derived on data with $b$ jets containing muons (tagged with the SLT method) and semileptonic $b$ decays in simulation. The TRFs are parameterized by the flavor, pseudorapidity and energy of the simulated jets. The overall weight for each event is changed by $P_{\text {event }}^{\text {taggable }} \cdot P_{\text {event }}^{\text {tag }}$ to account for $b$ tagging. 


\section{Selection and Reconstruction}

This section lists the event selection criteria used for this analysis. The event selection criteria are the requirements placed on the reconstructed objects to maximize the signal to background ratio in data. In addition, this section also explains an algorithm known as a kinematic fit, which is used to reconstruct events from data under the hypothesis of $t \bar{t}$ decay.

\subsection{Event Selection}

Most of the events in data collected by $\mathrm{D} \varnothing$ are entirely unrelated to $t \bar{t}$ decay. To isolate the events of interest, we start with the decay sequence $t \bar{t} \rightarrow W b W b \rightarrow$ lvqqbb. The result of this chain is a lepton with a large amount of momentum transverse to the beam pipe isolated from other particles, a neutrino and four or more jets. Because of the short lifetime and complex decay of the $\tau$ lepton, only electrons and muons are considered for this analysis. We require a large amount of missing transverse energy, $\mathbb{E}_{\mathrm{T}}$, to indicate the presence of the neutrino. We veto events with a second isolated electron or muon so that the data used in this analysis does not overlap with the data used by analyses in the di-lepton $(t \bar{t} \rightarrow W b W b \rightarrow l \nu l \nu b b)$ channel. Any object with the highest $\mathrm{p}_{\mathrm{T}}$ compared to objects of the same type is called the "leading" object. For example, if electron

$\# 1$ has $\mathrm{p}_{\mathrm{T}}=15 \mathrm{GeV}$, electron \#2 has $\mathrm{p}_{\mathrm{T}}=55 \mathrm{GeV}$ and electron $\# 3$ has $\mathrm{p}_{\mathrm{T}}=22$ $\mathrm{GeV}$, then electron \#2 is the leading electron.

The majority of the selection requirements are taken from the cross section measurement, Reference [24]. Additional criteria used in this analysis that are not imposed by the cross section measurement are the requirement of at least four 
jets, the requirement at least one jet to be $b$ tagged and the requirement of the convergence of the kinematic fit, described below.

The following criteria are required for events in both the $e+$ jets and the $\mu+$ jets channels:

- Luminosity block selection, i.e. removal of events from runs where data recording is considered to be poor;

- Good event quality (e.g. no excess noise in the calorimeter);

- At least three tracks associated with the primary vertex. The reconstructed primary vertex must have a z coordinate of $\left|z_{P V}\right|<60 \mathrm{~cm}$;

- At least four jets with $\mathrm{p}_{\mathrm{T}} \geq 20 \mathrm{GeV}$ and $\left|\eta_{\text {det }}\right|<2.5$ In RunIIb all jets are required to be vertex confirmed, i.e. at least two tracks in the jet cone must come from the primary vertex;

- At least one jet is required to have $\mathrm{p}_{\mathrm{T}} \geq 40 \mathrm{GeV}$. This requirement suppresses the $W+$ jets background;

- At least one jet with a $b$-tagging neural net value of 0.2 among the leading four jets;

- The kinematic fit must converge.

The selection requirements specific to the $e+$ jets channel are:

- One loose electron (Top_loose) with $\mathrm{p}_{\mathrm{T}} \geq 20 \mathrm{GeV}$ and $\left|\eta_{\text {det }}\right|<1.1$;

- The electron must originate from the primary vertex: $|\delta z(e, P V)|<1 \mathrm{~cm}$;

- For the tight data sample, where the measurement takes place, the electron is required to pass the tight isolation criteria (Top_tight);

- To ensure orthogonality to the dielectron channel, events with a second tight electron with $\mathrm{p}_{\mathrm{T}} \geq 15 \mathrm{GeV}$ and $\left|\eta_{\text {det }}\right|<2.5$ are rejected. Similarly, events with a tight isolated muon with $\mathrm{p}_{\mathrm{T}} \geq 15 \mathrm{GeV}$ and $\left|\eta_{\text {det }}\right|<2.0$ are rejected to ensure orthogonality with the electron-muon channel; 
- $\mathbb{E}_{\mathrm{T}} \geq 20 \mathrm{GeV}$;

- The "triangle cut", which is used to reject multijet events: $\Delta \phi\left(e, \mathbb{E}_{\mathrm{T}}\right)>$ $2.2-0.045 \cdot \mathbb{E}_{\mathrm{T}}$;

- Events in data are required to have passed the $e+$ jets "superOR" trigger, which is a combination of the single electron OR and $e+$ jets OR triggers.

The selection requirements specific to the $\mu+$ jets channel are:

- At least one loose isolated ( $\Delta R$ isolation) muon with $\mathrm{p}_{\mathrm{T}} \geq 20 \mathrm{GeV}$ and $\left|\eta_{\text {det }}\right|<2.0$

- The muon must originate from the primary vertex: $|\delta z(\mu, P V)|<1 \mathrm{~cm}$;

- For the tight data sample, where the measurement takes place, the leading muon is required to pass the tight isolation criteria (TopP14);

- To reject events from $Z \rightarrow \mu \mu+$ jets decays, the invariant masses $m_{\mu \mu}$ of the leading muon and any other loose isolated muons with $\mathrm{p}_{\mathrm{T}} \geq 15 \mathrm{GeV}$ and $\left|\eta_{\text {det }}\right|<2.0$ must be $70 \mathrm{GeV}<\mathrm{m}_{\mu \mu}<110 \mathrm{GeV}$, away from the peak of the $Z$ boson mass spectrum;

- $\mathbb{E}_{\mathrm{T}} \geq 25 \mathrm{GeV}$ and $\mathbb{E}_{\mathrm{T}}<250 \mathrm{GeV}$;

- To ensure orthogonality to the dimuon channel, events with a second muon with $\mathrm{p}_{\mathrm{T}} \geq 15 \mathrm{GeV}$, medium $n_{\text {seg }}=3$ and medium track quality are rejected. Similarly, events with a tight isolated electron with $\mathrm{p}_{\mathrm{T}} \geq 15 \mathrm{GeV}$ and $\left|\eta_{\text {det }}\right|<$ 2.5 are rejected to ensure orthogonality with the electron-muon channel;

- $\mathbb{E}_{\mathrm{T}} \geq 20 \mathrm{GeV}$;

- The "triangle cut", which is used to reject multijet events: $\Delta \phi\left(\mu, \mathbb{E}_{\mathrm{T}}\right)>$ $2.1-0.035 \cdot \mathbb{E}_{\mathrm{T}}$

- $\mathrm{wmt}=\sqrt{\left(\mathrm{p}_{\mathrm{T}}^{l}+\mathbb{E}_{\mathrm{T}}\right)^{2}-\left(p_{x}^{l}+\mathbb{E}_{\mathrm{x}}\right)^{2}-\left(p_{y}^{l}+\mathbb{E}_{\mathrm{y}}\right)^{2}}<250 \mathrm{GeV}$

- Events in data collected during Run IIa are required to have passed the $\mu+$ jets "superOR" trigger, which is a combination of the single muon OR 
and $\mu+$ jets OR triggers. Due to issues with the $\mu+$ jets OR triggers, events in Run IIb are required to have passed at least one of the single muon triggers, known collectively as the single muon OR trigger.

\subsection{Matrix Method for Multijet Background De- termination}

Events from the multijet background are taken directly from data, rather than from simulation. In order to obtain a sample of events that does not overlap with the sample used for the measurement, we use the so-called "Matrix Method." Two different samples are used, one with loose lepton isolation criteria and one with tight lepton isolation criteria. Data used for the measurement are contained in the tight sample, which is a subset of the loose sample. Data from the loose sample that do not pass the tight selection criteria are used to estimate the contributions from multijet background. This sample is known as the loose-minus-tight sample, as together the tight sample and the loose-minus-tight sample make up the loose sample.

Top quark decays, as well as decays from the $W+$ jets background, contain true isolated leptons. We refer to these decays as "signal" decays, because the lepton comes from signal-like events. The number of signal-like events passing the loose isolation criteria is $N^{s i g}$. The number of multijet events that do not contain truly isolated leptons, but still pass the loose isolation criteria, is $N^{\mathrm{MJ}}$. The number of events passing the loose isolation criteria, $n_{l}$, and the number of events passing the tight isolation criteria, $n_{t}$ can be written as a matrix equation:

$$
\left(\begin{array}{c}
n_{l} \\
n_{t}
\end{array}\right)=\left(\begin{array}{cc}
1 & 1 \\
\epsilon_{s i g} & \epsilon_{Q C D}
\end{array}\right)\left(\begin{array}{l}
N^{s i g} \\
N^{\mathrm{MJ}}
\end{array}\right)
$$

The quantities $\epsilon_{s i g}$ and $\epsilon_{Q C D}$ are the efficiencies for signal-like and multijet events from the loose sample pass in the tight isolation criteria. Table 6.1 lists the values of $\epsilon_{s i g}$ and $\epsilon_{Q C D}$ for each channel.

The matrix from Equation 6.1 is inverted to obtain the number of events for $N^{s i g}$ and $N^{\mathrm{MJ}}$ in the loose sample: 


$$
\begin{gathered}
N^{s i g}=\frac{n_{t}-\epsilon_{Q C D} n_{l}}{\epsilon_{s i g}-\epsilon_{Q C D}} \text { and } N^{\mathrm{MJ}}=\frac{\epsilon_{s i g} n_{l}-n_{t}}{\epsilon_{\text {sig }}-\epsilon_{Q C D}} . \\
\begin{array}{ccc}
e+\text { jets } & \mu+\text { jets } \\
\hline & \multicolumn{3}{c}{\text { Run IIa }} \\
\hline \epsilon_{\text {sig }} 0.831 \pm 0.011 & 0.881 \pm 0.039 \\
\hline \epsilon_{Q C D} & 0.109 \pm 0.008 & 0.172 \pm 0.048 \\
\hline \epsilon_{\text {sig }} & 0.813 \pm 0.045 & 0.896 \pm 0.021 \\
\epsilon_{Q C D} & 0.124 \pm 0.015 & 0.219 \pm 0.043
\end{array}
\end{gathered}
$$

Run IIb

Table 6.1 Efficiencies for isolated leptons and mis-identified jets to pass the tight selection criteria. The uncertainties include both systematic and statistical contributions [24].

To determine $\epsilon_{Q C D}$, events with $\mathbb{E}_{\mathrm{T}}<10 \mathrm{GeV}$ that otherwise pass the selection criteria are taken from data [24]. This sample is enriched in multijet events, and are assumed to include no truly isolated leptons. The number of events in this sample passing tight selection criteria are divided by the number of events passing the loose selection criteria, yielding $\epsilon_{Q C D}$.

A similar procedure calculates $\epsilon_{\text {sig }}$, except that data are replaced by simulated $W+$ jets and $t \bar{t}$ events. The value of $\epsilon_{\text {sig }}$ is determined from the mean of the separate $W+$ jets and $t \bar{t}$ calculations. However, these samples have differing kinematic distributions and the differences between the average $\epsilon_{\text {sig }}$ and $\epsilon_{\text {sig }}$ from the pure $W+$ jets and $t \bar{t}$ samples are assigned as systematic uncertainties. The usage of the Matrix Method in our analysis, described in more detail in Section 7.2.1, is slightly different than described here.

\subsection{Constrained Kinematic Fit}

After selection, we are left with four or more jets, a lepton and missing transverse energy, but still no information about the kinematics of the top quarks. To find the decay angles and energies of the top quarks, the three jets from the decay of the hadronic top must be found. Additionally, the jets that originated from the light quarks from the decay of the $W$ boson from the decay of the hadronic top 
also need to be identified. To do this we need to answer the question: Which jets belong to which quarks (partons)? Figure 6.1 illustrates this issue.

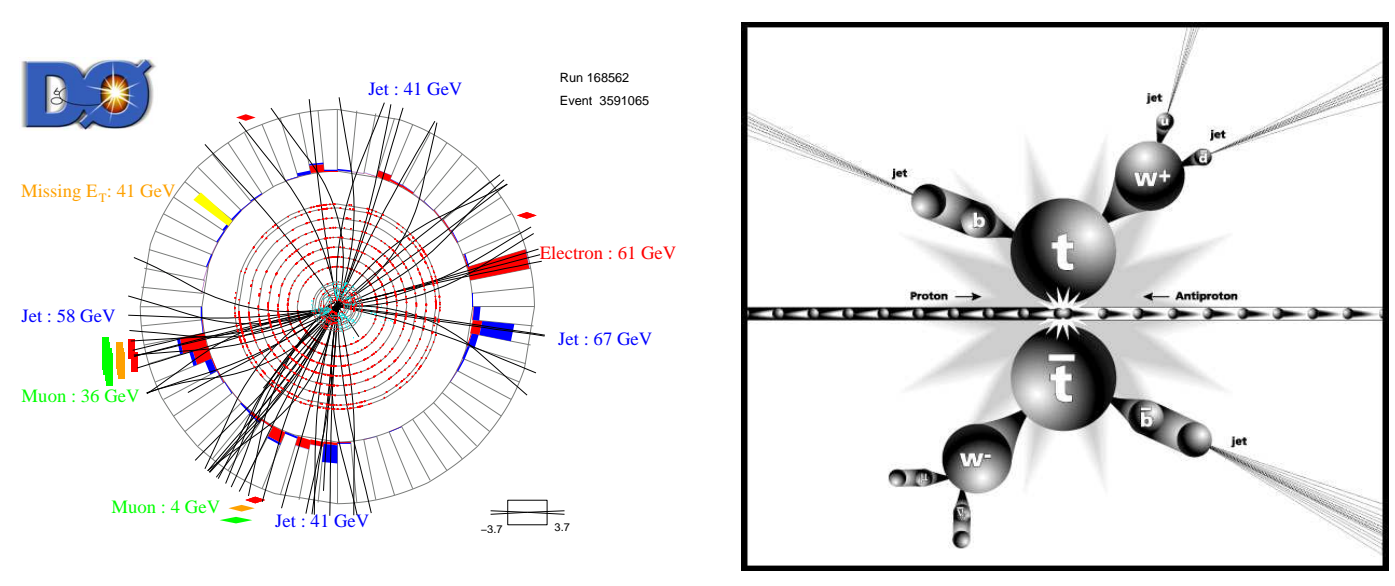

Figure 6.1 Which jets originate from which quarks? DØ event display of toplike $e+$ jets event (left) and rendition of $t \bar{t}$ decay to lepton+jets (right) [99].

By requiring one $b$-tagged jet to be assigned as $b$ quark and only considering the leading four jets, the total number of combinations is reduced from twelve to six, as shown in Table 6.2, where $b_{1}$ is the $b$-tagged jet and $j_{1}, j_{2}$ and $j_{3}$ are the other jets. If there are two $b$-tagged jets, $b_{1}$ and $b_{2}$, the number of combinations is reduced even further to two, as shown in Table 6.3. For the case of more than two $b$-tagged jets, the situation is essentially the same as the case of one $b$-tagged jet and the number of jet-parton combinations is six.

\begin{tabular}{c|c|c}
$b$ from $t \rightarrow W b \rightarrow l \nu b$ & $b$ from $t \rightarrow W b \rightarrow b q q$ & $q q$ from $t \rightarrow W b \rightarrow b q q$ \\
\hline$b_{1}$ & $j_{1}$ & $j_{2}, j_{3}$ \\
$b_{1}$ & $j_{2}$ & $j_{1}, j_{3}$ \\
$b_{1}$ & $j_{3}$ & $j_{1}, j_{2}$ \\
$j_{1}$ & $b_{1}$ & $j_{2}, j_{3}$ \\
$j_{2}$ & $b_{1}$ & $j_{1}, j_{3}$ \\
$j_{3}$ & $b_{1}$ & $j_{1}, j_{2}$
\end{tabular}

Table 6.2 Table of potential jet-parton assignments for the reconstructed top quarks for events with one $b$ tag.

To solve these issues, we use a kinematic fit referred to inside the $\mathrm{D} \varnothing$ top group as HITFIT [100]. The kinematic fitting algorithm was originally designed 


\begin{tabular}{c|c|c}
$b$ from $t \rightarrow W b \rightarrow l \nu b$ & $b$ from $t \rightarrow W b \rightarrow b q q$ & $q q$ from $t \rightarrow W b \rightarrow b q q$ \\
\hline$b_{1}$ & $b_{2}$ & $j_{1}, j_{1}$ \\
$b_{2}$ & $b_{1}$ & $j_{1}, j_{2}$
\end{tabular}

Table 6.3 Table of potential jet-parton assignments for the reconstructed top quarks for events with two $b$ tags.

to measure the mass of the top quark, which is well measured now, so we can use it as an additional constraint. For this analysis we use the kinematic fit to figure out the jet-parton assignment, which is the assignment of jets in the detector to daughter quarks from the $t \bar{t}$ decay. The general idea of the kinematic fit is to use known information about the top decays, such as the mass of the $t$ quark (172.5 $\mathrm{GeV}$ ) and the mass of the $W$ boson $(80.4 \mathrm{GeV})$, and figure out which jet-parton assignment from the lists in Tables 6.2 and 6.3 fits the $t \bar{t}$ hypothesis the best.

To find the best jet-parton assignment, the following equation is minimized:

$$
\chi^{2}=\left(\vec{x}-\vec{x}_{\text {meas }}\right)^{T} \mathbf{G}\left(\vec{x}-\vec{x}_{\text {meas }}\right),
$$

where $\vec{x}$ is the vector of the fit variables, $\vec{x}_{\text {meas }}$ is the vector of the measured variables and $\mathbf{G}$ is the inverse of the error matrix. The resolutions on each of the fit variables are determined by comparing simulated particles processed by the detector simulation with their counterpart objects output by the reconstruction sequences. The following constraints are imposed on the kinematic fit:

$$
\begin{aligned}
& m_{W, \text { had }}=m_{W, \text { lep }}=80.4 \mathrm{GeV}, \\
& m_{t, \text { had }}=m_{t, \text { lep }}=172.5 \mathrm{GeV} .
\end{aligned}
$$

Because the constraints are non-linear combinations of the fit variables, a numerical approach is used to minimize the constrained equation. The solution with the lowest $\chi^{2}$ is considered to be the "correct" solution, i.e. the jets and $t \bar{t}$ daughter particles are properly matched. This solution is the correct solution in about $70 \%$ of simulated $t \bar{t}$ events that have one $b$ tag and are "matchable." Events that are matchable have all four partons separated by a distance of 0.5 in $\eta$ and $\phi$. Otherwise the partons from the simulated top quark decays overlap and the 
jets in the event cannot be matched. As seen in Figure 6.2, less than half of $t \bar{t}$ decays are matchable. For events with two $b$-tagged jets, the kinematic fit finds the solution more than $85 \%$ of the time. If the mass constraint is removed and the top masses are set to be equal, but not fixed to a set value, the performance of the kinematic fit drops and the correct solution is found at a lower rate $(\sim 55 \%$ for events with one $b \mathrm{tag}$ ). The kinematic fit fails to converge for approximately $1 \%$ of events. This fraction is the same for both data and simulation. The number of degrees of freedom for the fit is about three, found by fitting a $\chi^{2}$ function to the distribution in Figure 6.2.

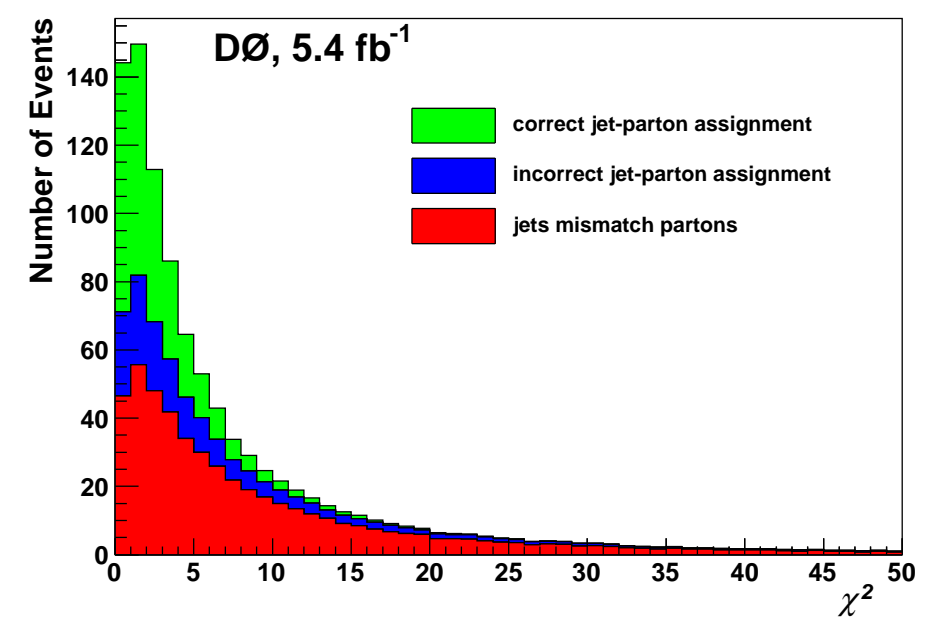

Figure 6.2 $\chi^{2}$ distribution from the kinematic fit for $t \bar{t}$ signal events.

Note: Many quantities in the analysis, such as $\Delta y$ and $m_{t \bar{t}}$, the invariant mass of the $t \bar{t}$ system, are constructed using variables output from the kinematic fit $(\vec{x})$, and not the quantities reconstructed in the detector $\left(\vec{x}_{\text {meas }}\right)$. 


\section{Analysis Technique and Results}

This chapter is the main portion of the thesis and the section the author, as the main analyzer, had the largest role in shaping. All aspects of the analysis will be discussed in this section.

\subsection{Predicting reconstruction-level asymmetries}

The main procedure we use to make predictions is quite simple. We take the distributions in $\Delta y$ and $q_{l} y_{l}$ from MC@NLO+HERWIG after the simulated events are processed by simulation of the $\mathrm{D} \emptyset$ detector and reconstructed with the selection criteria described earlier. The predicted reconstruction-level asymmetries are found from these distributions by simply counting the number of (weighted) events with $\Delta y\left(\right.$ or $\left.\mathrm{q}_{1} \mathrm{y}_{1}\right)>0$ and $\Delta y\left(\right.$ or $\left.\mathrm{q}_{1} \mathrm{y}_{\mathrm{l}}\right)<0$. Table 7.1 lists the predicted $\Delta y$ - and lepton-based asymmetries by channel and data collection period.

\subsection{Reconstruction-level measurements}

In this section, the technique for measuring the reconstruction level asymmetries is described. By our definition of reconstruction-level, this quantity is the asymmetry remaining in the data sample after the backgrounds have been subtracted, so the asymmetry from the top quarks in the data sample. In addition to measuring the asymmetry, the signal and background fractions need to be measured. As described in Section 6.3, all of the kinematic quantities used for the reconstructionlevel measurements are taken from the solution to the kinematic fit with the lowest $\chi^{2}$. 


\begin{tabular}{|c|c|c|c|}
\hline \multicolumn{2}{|c|}{ Sample } & \multirow{2}{*}{$\Delta y$-based $A_{\mathrm{FB}}(\%)$} & lepton-based $A_{\mathrm{FB}}(\%)$ \\
\hline Reco. Version & Channel & & $1.1 \pm 0.3$ \\
Combined & Combined & $2.4 \pm 0.3$ & $0.9 \pm 0.4$ \\
& $e+$ jets & $2.4 \pm 0.4$ & $1.5 \pm 0.4$ \\
\hline \multirow{3}{*}{ Run IIb } & $\mu+$ jets & $2.5 \pm 0.4$ & $0.8 \pm 0.4$ \\
& Combined & $2.2 \pm 0.4$ & $0.7 \pm 0.5$ \\
& $e+$ jets & $2.3 \pm 0.5$ & $1.1 \pm 0.5$ \\
\hline \multirow{3}{*}{ Run IIa } & Combined & $2.0 \pm 0.5$ & $2.0 \pm 0.4$ \\
& $e+$ jets & $2.5 \pm 0.6$ & $1.5 \pm 0.6$ \\
& $\mu+$ jets & $3.8 \pm 0.6$ & $2.6 \pm 0.6$ \\
\hline
\end{tabular}

Table 7.1 Predictions from MC@NLO. The quoted uncertainties are statistical, from the size of the MC sample.

\subsubsection{Template Fit and Likelihood Discriminant}

To measure the asymmetry, signal events need to be separated from background events. The overall asymmetry in data can be presented as:

$$
A_{\mathrm{FB}}^{\text {data }}=\left(\frac{N_{\text {sig }}}{N_{\text {data }}} A_{\mathrm{FB}}^{\text {sig }}+\Sigma_{i}^{N_{b g}} \frac{N_{b g_{i}}}{N_{\text {data }}} A_{\mathrm{FB}}^{b g_{i}}\right)
$$

Multiplying both sides by $\frac{N_{\text {data }}}{N_{\text {sig }}}$ and shuffling terms around, the asymmetry from the signal can be found by subtracting known backgrounds from data:

$$
A_{\mathrm{FB}}^{s i g}=\left(\frac{N_{\text {data }}}{N_{\text {sig }}} A_{\mathrm{FB}}^{\text {data }}-\Sigma_{i}^{N_{b g}} \frac{N_{b g_{i}}}{N_{s i g}} A_{\mathrm{FB}}^{b g_{i}}\right) .
$$

There are multiple parts of Equation 7.2 that need to be measured. Both $N_{\text {data }}$ and $A_{\mathrm{FB}}^{\text {data }}$ are taken directly from raw data, after the final selection. The predicted asymmetries from the backgrounds processes, $A_{\mathrm{FB}}^{b g_{i}}$, are found in different ways. The asymmetry for the $W+$ jets background, $A_{\mathrm{FB}}^{W+j e t s}$, is taken directly from the ALPGEN simulation. For the MJ background, the predicted asymmetry, $A_{\mathrm{FB}}^{\mathrm{MJ}}$, is taken from the "loose-tight" data sample.

The remaining piece is the sample composition, which consists of $N_{t \bar{t}}$, the number of signal events, $N_{W+\text { jets }}$, the number of background events, and $N_{\mathrm{MJ}}$, the number of multijet events. The number of MJ events is constrained using 
the "matrix" method, which works by solving two equations with two unknown quantities. The total number of events in the sample with the loose lepton isolation requirements is:

$$
N_{l}=N_{l}^{t \bar{t}}+N_{l}^{W+j e t s}+N_{l}^{M J}
$$

Similarly, the total number of events passing the tight lepton isolation is:

$$
N_{t}=\epsilon_{s i g}\left(N_{l}^{t \bar{t}}+N_{l}^{W+j e t s}\right)+\epsilon_{Q C D}\left(N_{l}^{M J}\right) .
$$

For the tight sample, two different efficiencies play a role. One is the efficiency for leptons from $W$ decay to pass the tight isolation criteria, $\epsilon_{s i g}$. The other is the efficiency for MJ events to have a jet faking a lepton, $\epsilon_{Q C D}$. Because the leptons arise from such different types of decay, this parameterization is useful in figuring out the ratio between MJ events and events with real isolated leptons. Sample with different selection criteria are used to measure $\epsilon_{s i g}$ and $\epsilon_{Q C D}$. In some analyses, these two equations are solved to give $N_{t}^{t \bar{t}}+N_{t}^{W+j e t s}$ and $N_{t}^{Q C D}$ in terms of the counts in data and the efficiencies. In this analysis, these equations are used as constraints.

The distribution for the discriminant and the asymmetry for the MJ sample are found using the loose-tight (L-T) sample, a sample of events that pass loose lepton isolation requirements but fail the tight criteria used in the measurement. The underlying assumption is the change in lepton isolation will not change the overall kinematics very much. The number of events in the loose minus tight sample in terms of the composition of the loose sample and the lepton efficiencies is:

$$
N_{l-t}=\left(1-\epsilon_{s i g}\right)\left(N_{l}^{t \bar{t}}+N_{l}^{W+j e t s}\right)+\left(1-\epsilon_{Q C D}\right)\left(N_{l}^{M J}\right)
$$

The number of events in the loose minus tight sample can also be shown in terms of the composition of the tight sample by replacing $N_{l}^{t \bar{t}}+N_{l}^{W+j e t s}$ with $\frac{\left(N_{t}^{t \bar{t}}+N_{t}^{W+j e t s}\right)}{\epsilon_{s i g}}$ and $N_{l}^{M J}$ with $\frac{N_{t}^{M J}}{\epsilon_{Q C D}}$ :

$$
N_{l-t}=\left(\frac{1-\epsilon_{s i g}}{\epsilon_{s i g}}\right)\left(N_{t}^{t \bar{t}}+N_{t}^{W+j e t s}\right)+\left(\frac{1-\epsilon_{Q C D}}{\epsilon_{Q C D}}\right)\left(N_{t}^{M J}\right)
$$


When using the L-T sample as the source for the MJ background, some additional $t \bar{t}$ and $W$ +jets events are included with in the MJ template, since the L-T sample is not entirely composed of multijet events. The number of multijet events in the $\mathrm{L}-\mathrm{T}$ sample is $N_{l-t}^{M J}$. When the $\mathrm{L}-\mathrm{T}$ sample is used as a background template in the ML fit, the number of fitted multijet events is $N_{t}^{M J}$. So the L-T sample need to be multiplied by a factor of $a$, so that

$$
a N_{l-t}^{M J}=N_{t}^{M J}
$$

Combining Equations 7.7 and 7.15 leads to:

$$
a\left(\frac{1-\epsilon_{Q C D}}{\epsilon_{Q C D}}\right) N_{t}^{M J}=N_{t}^{M J}
$$

And so $a$ is:

$$
a=\left(\frac{\epsilon_{Q C D}}{1-\epsilon_{Q C D}}\right)
$$

There are now $\left(\frac{\epsilon_{Q C D}}{1-\epsilon_{Q C D}}\right) N_{l-t}$ events in the fitted L-T template, so that the multijet portion of the L-T template is equal to the number of multijet events fitted in the tight sample. This means that the number of $t \bar{t}$ and $W+$ jets events in the fitted template made from the L-T sample is:

$$
a N_{l-t}^{t \bar{t}+W+j e t s}=\left(\frac{\epsilon_{Q C D}}{1-\epsilon_{Q C D}} \frac{1-\epsilon_{s i g}}{\epsilon_{s i g}}\right) N_{t}^{t \bar{t}+W+j e t s}=C\left(N_{t}-N_{t}^{M J}\right)
$$

So the contamination factor $\mathrm{C}$ is:

$$
C=\frac{\epsilon_{Q C D}}{1-\epsilon_{Q C D}} \frac{1-\epsilon_{s i g}}{\epsilon_{s i g}}
$$

We use a maximum likelihood (ML) fit to measure the asymmetry and sample composition with the templates. Using the procedure from Ref. [1], which is based on the template fit used in Ref. [101], the following likelihood is constructed: 


$$
L\left(N_{t}^{t \bar{t}}, N_{t}^{W+j e t s}, N_{t}^{\mathrm{MJ}}, A_{\mathrm{FB}}\right)=\left[\prod_{i} P\left(n_{i}^{o b s}, \mu_{i}\right)\right] \cdot P\left(N_{l-t}^{o b s}, N_{l-t}\right)
$$

The likelihood equation contains the constraint from the number of L-T events. The additional constraint that $N_{t}=N_{t}^{t \bar{t}}+N_{t}^{W+j e t s}+N_{t}^{\mathrm{MJ}}$ is also included, which reduces Equation 7.12 to $L\left(N_{t}^{t \bar{t}}, N_{t}^{\mathrm{MJ}}, A_{\mathrm{FB}}\right)$, as $N_{t}^{W+j e t s}=N_{t}-N_{t}^{t \bar{t}}-N_{t}^{\mathrm{MJ}}$. Each template includes a binned distribution in the discriminant as well as a bin with the fraction of forward events and a bin with the fraction of backward events.

To deal with the binned templates, a ML fit for binned data is used $[102,103]$. Since the bin contents are independent of each other, the probability for $n_{i}^{o b s}$ is taken from the Poisson distribution, $P\left(n_{i}^{o b s}, \mu_{i}\right)=\frac{\mu_{i}^{n_{i}^{o b s}}}{n_{i}^{o b s} !} e^{-\mu_{i}}$. Taking the logarithm of Equation 7.12 leads to

$$
\log \left(\prod_{i} P\left(n_{i}^{o b s}, \mu_{i}\right)\right)=\sum_{i}\left(n_{i}^{o b s} \log \left(\mu_{i}\right)-\mu_{i}-\log \left(n_{i}^{o b s} !\right)\right)
$$

The negative logarithm of the likelihood, $-\log (L)$, is minimized using the TMinuit class from ROOT [104].

The overall structure of the likelihood equation is:

$$
-\log (L)=\sum_{i}^{n_{b i n}}\left(-n_{i}^{o b s} \log \left(\mu_{i}\right)+\mu_{i}\right)-N_{l-t}^{o b s} \log \left(N_{l-t}\right)+N_{l-t}
$$

where $n_{i}^{o b s}$ is the number of data events in bin $i$ and $\mu_{i}$ is predicted number of events in bin $i$ and $N_{l-t}$ is defined as:

$$
N_{l-t}=\left(\frac{1-\epsilon_{s i g}}{\epsilon_{s i g}}\right)\left(N_{t}-N_{t}^{M J}\right)+\left(\frac{1-\epsilon_{Q C D}}{\epsilon_{Q C D}}\right)\left(N_{t}^{M J}\right)
$$

The bin contents $n_{i}^{\text {obs }}$ from Equation 7.14 are taken from the template made with the tight data sample. The one part of Equation 7.14 left to explain is $\mu_{i}$. The parameters of the fit are contained within $\mu_{i}$, which uses the equalities $N_{F}=N_{t \bar{t}} \frac{1+A_{\mathrm{FB}}}{2}$ and $N_{B}=N_{t \bar{t}} \frac{1-A_{\mathrm{FB}}}{2}$. The signal contamination factor derived in 
Equation 7.11 is also included, with a factor of $(1-C)$ for the standard $t \bar{t}$ and $W+$ jets templates. The $\mu_{i}$ term for the $i$ th bin is defined as:

$$
\begin{aligned}
\mu_{i}\left(N_{t}^{t \bar{t}}, N_{t}^{\mathrm{MJ}}, A_{\mathrm{FB}}\right) & =f_{i}^{t \bar{t}(\Delta y>0)} N_{t}^{t \bar{t}}\left(\frac{1+A_{\mathrm{FB}}}{2}\right)(1-C) \\
& +f_{i}^{t \bar{t}(\Delta y<0)} N_{t}^{t \bar{t}}\left(\frac{1-A_{\mathrm{FB}}}{2}\right)(1-C) \\
& +f_{i}^{W+j e t s}\left(N_{t}-N_{t}^{t \bar{t}}-N_{t}^{\mathrm{MJ}}\right)(1-C) \\
& +f_{i}^{l-t}\left(N_{t}^{\mathrm{MJ}}+C\left(N_{t}-N_{t}^{\mathrm{MJ}}\right)\right)
\end{aligned}
$$

where $f_{i}^{t \bar{t}(\Delta y>0)}, f_{i}^{t \bar{t}(\Delta y<0)}, f_{i}^{W+j e t s}$ and $f_{i}^{\mathrm{MJ}}$ are the fractions of events in the $i$ th bin. The templates are normalized to one:

i. Forward signal template: the fraction of forward events is set to 1 and the fraction of backward events is set to 0 . The discriminant is made from all signal events.

ii. Backward signal template: the fraction of forward events is set to 0 and the fraction of backward events is set to 1 . The discriminant is made from all signal events.

iii. $W+$ jets background template: the fraction of forward events and the fraction of backward events are taken from simulation.

iv. L-T background template: the fraction of forward events and the fraction of backward events are taken from data.

To separate $W+$ jets and $t \bar{t}$ events, a "likelihood" discriminant (LD) is used [105]. Various distributions in kinematic variables are combined to increase the overall strength at differentiating signal events $(S)$ from background events $(B)$ using the following equation:

$$
L D=\frac{\exp \left(\sum_{i=1}^{N_{\text {var }}} \ln \left(\frac{S}{B}\right)_{i}^{f i t}\right)}{\exp \left(\sum_{i=1}^{N_{\text {var }}} \ln \left(\frac{S}{B}\right)_{i}^{f i t}\right)+1}
$$


where the term $\ln \left(\frac{S}{B}\right)_{i}^{f i t}$ represents a function fit to a template made with signal and background simulations for the $i$ th variable ( $N_{\text {var }}$ variables in total $)$, and $\mathrm{S}$ and $\mathrm{B}$ are the probabilities for simulated events from signal and background processes to have a specific value of variable $i$. Appendix $\mathrm{C}$ contains the input functions for the discriminants for both periods of data collection. If an event is signal-like, the numerator and the denominator will quickly become large, resulting in a value of 1. In the opposite case, the numerator would go to zero and the result would be 0 . In this way, the discriminant is able to combine multiple pieces of information together to distinguish between events from signal and background processes.

Four different variables, shown in Figure 7.1, are used in the discriminant:

i. $p_{T}^{l b}$, the transverse momentum of the leading $b$-tagged jet, which is the $b$-tagged with the largest $p_{T}$,

ii. $M_{j j}$, the invariant mass of the two jets assigned to the hadronic $W$ by the kinematic fit,

iii. $k_{T}^{m i n}, \min \left(\mathrm{p}_{\mathrm{T}}^{1}, \mathrm{p}_{\mathrm{T}}^{2}\right) \Delta \mathrm{R}^{12}$, where $\Delta R^{12}$ is the minimum separation in $\eta$ - $\phi$ between any two of the leading four jets,

iv. $\chi^{2}$ of the jet assignment chosen by the kinematic fit, so the minimum $\chi^{2}$.

\subsubsection{Results for the $\Delta y$-based asymmetry}

To find the asymmetry in $\Delta y$ reconstructed for $t \bar{t}$ events, the maximum likelihood template fit described earlier is used. Recall that the fit has three free parameters: the number of $t \bar{t}$ events, the number of MJ events and $A_{\mathrm{FB}}$ for $t \bar{t}$ events. The number of $W+$ jets events is constrained so that the total sum $\left(N_{\mathrm{MJ}}+N_{W+\text { jets }}+\right.$ $N_{t \bar{t}}$ ) equals the number of events in data. For the $\Delta y$-based reconstruction level asymmetry, forward events have $\Delta y>0$ and backward events have $\Delta y<0$.

The results from the maximum likelihood fit are shown in Table 7.2. The table presents the measured sample composition and asymmetry for the entire sample and for different channels: $e+$ jets, $\mu+$ jets, $l+4$ jets and $l+\geq 5$ jets. 

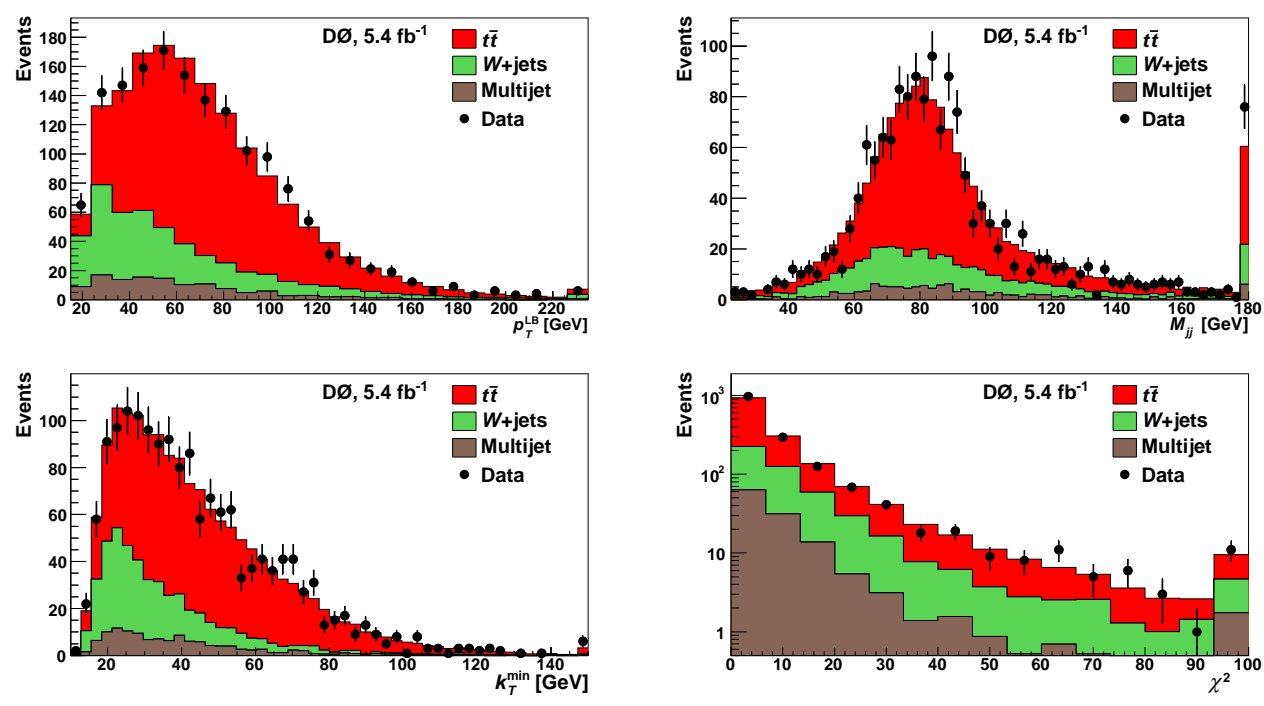

Figure 7.1 Comparison of data and simulation for the four variables used to construct the discriminant.
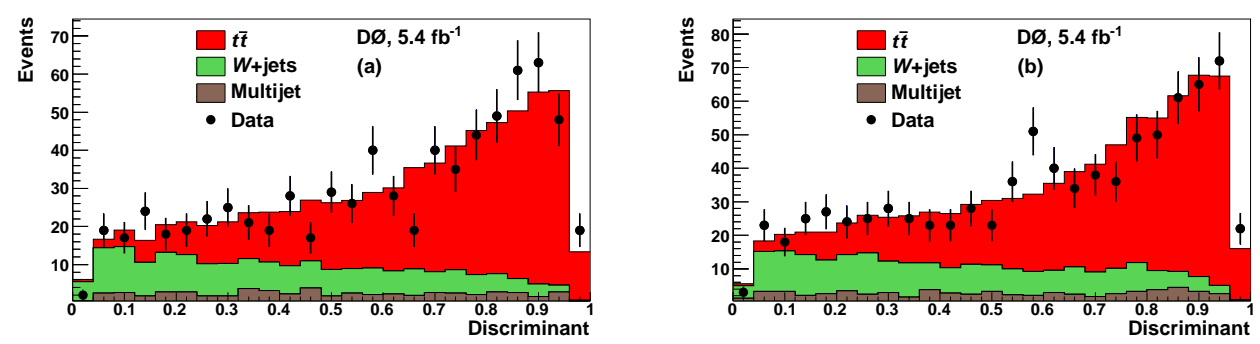

Figure 7.2 Distributions in the discriminant for $\Delta y<0$ (a) and $\Delta y>0$ (b).

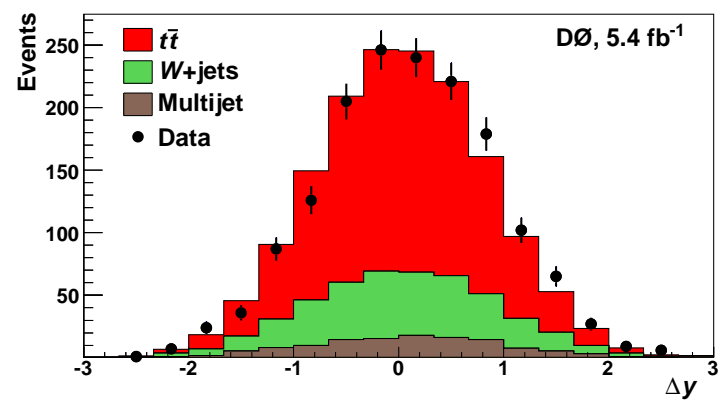

Figure 7.3 The $\Delta y$ distribution for the data, shown with the fitted sample composition. 


\begin{tabular}{|l|c|c|c|c|c|}
\hline & $l+\geq 4$ jets & $e+\geq 4$ jets & $\mu+\geq 4$ jets & $l+4$ jets & $l+\geq 5$ jets \\
\hline$N$ & 1581 & 852 & 729 & 1314 & 267 \\
$N_{\Delta y>0}$ & 849 & 455 & 394 & 717 & 132 \\
$N_{\Delta y<0}$ & 732 & 397 & 335 & 597 & 135 \\
\hline$N_{t \bar{t}}$ & $1126 \pm 39$ & $613 \pm 27$ & $506 \pm 27$ & $902 \pm 36$ & $218 \pm 16$ \\
$N_{W}$ & $376 \pm 39$ & $159 \pm 27$ & $223 \pm 27$ & $346 \pm 36$ & $35 \pm 16$ \\
$N_{\mathrm{MJ}}$ & $79 \pm 5$ & $80 \pm 4$ & $0 \pm 0$ & $66 \pm 4$ & $13 \pm 2$ \\
\hline Data $A_{\mathrm{FB}}(\%)$ & $9.2 \pm 3.6$ & $8.6 \pm 4.9$ & $9.0 \pm 5.5$ & $12.2 \pm 4.2$ & $-3.0 \pm 7.8$ \\
\hline MC@NLO $A_{\mathrm{FB}}(\%)$ & $2.4 \pm 0.3$ & $2.4 \pm 0.4$ & $2.5 \pm 0.4$ & $3.9 \pm 0.3$ & $-2.9 \pm 0.7$ \\
\hline
\end{tabular}

Table 7.2 Sample sizes and $A_{\mathrm{FB}}$ fit results. Only statistical uncertainties are included.

In Figure 7.2, the data and fitted templates for the discriminants for both forward and backward events are shown. Figure 7.3 shows the $\Delta y$ distribution for the entire dataset.

Except for the $l+\geq 5$ jets channel, all of the measured asymmetries in Table 7.2 are larger by more than one standard deviation from the asymmetries predicted by MC@NLO. The main result of $A_{\mathrm{FB}}=(9.2 \pm 3.6) \%$ is almost two standard deviations larger than the predicted result of $(2.4 \pm 0.3) \%$. The $e+$ jets and $\mu+$ jets channels are in good agreement with each other. The nominal value from the fit for the $l+\geq 5$ jets channel is in very good agreement with the prediction, but the uncertainty is large.

\subsubsection{Results for the lepton-based asymmetry}

The maximum likelihood fit that was described earlier measures the forwardbackward asymmetry of the $q_{l} y_{l}$ distribution. For the lepton-based asymmetry, forward events have $q_{l} y_{l}>0$ and backward events have $q_{l} y_{l}<0$. Because of issues with the selection efficiency at large $y_{l}$, which will be described in more detail later, an additional requirement of $\left|y_{l}\right|<1.5$ is imposed. This requirement slightly reduces the overall number of events in the tight sample from 1581 to 1532 .

The results for the lepton-based measurements are shown in Table 7.3. Figure 7.4 shows the data and the fitted template distributions in $q_{l} y_{l}$. 


\begin{tabular}{|l|c|c|c|c|c|}
\hline & $l+\geq 4$ jets & $e+\geq 4$ jets & $\mu+\geq 4$ jets & $l+4$ jets & $l+\geq 5$ jets \\
\hline$N$ & 1532 & 852 & 680 & 1276 & 256 \\
$N_{q \cdot y_{l}>0}$ & 867 & 485 & 382 & 730 & 137 \\
$N_{q \cdot y_{l}<0}$ & 665 & 367 & 298 & 546 & 119 \\
\hline$N_{t \bar{t}}$ & $1096 \pm 39$ & $613 \pm 27$ & $478 \pm 27$ & $881 \pm 36$ & $211 \pm 16$ \\
$N_{W}$ & $356 \pm 39$ & $159 \pm 27$ & $202 \pm 27$ & $323 \pm 36$ & $31 \pm 16$ \\
$N_{\mathrm{MJ}}$ & $79 \pm 5$ & $80 \pm 4$ & $0 \pm 0$ & $66 \pm 4$ & $14 \pm 2$ \\
\hline Data $A_{\mathrm{FB}}^{l}(\%)$ & $14.2 \pm 3.6$ & $16.8 \pm 4.9$ & $9.7 \pm 5.5$ & $16.2 \pm 4.2$ & $6.0 \pm 7.8$ \\
\hline $\mathrm{MC} @ \mathrm{NLO} A_{\mathrm{FB}}^{l}(\%)$ & $0.8 \pm 0.3$ & $0.7 \pm 0.4$ & $1.0 \pm 0.4$ & $2.1 \pm 0.3$ & $-3.8 \pm 0.7$ \\
\hline
\end{tabular}

Table 7.3 Result of fit for sample composition and lepton-based reconstructionlevel $A_{\mathrm{FB}}^{l}$. Only statistical uncertainties are included.

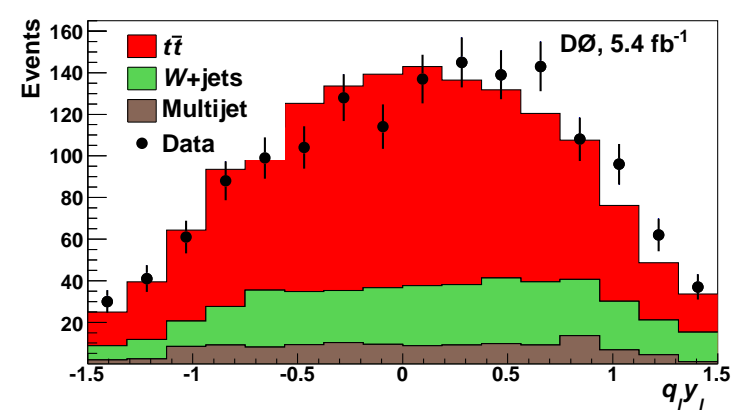

Figure 7.4 The $q_{l} y_{l}$ distribution for data, shown with the fitted sample composition. 
The measured lepton-based asymmetries are larger than the predicted asymmetries by multiple standard deviations. The result of the $l+\geq 4$ jets channel is $(14.2 \pm 3.6) \%$ is more than three standard deviations larger than the prediction of $(0.8 \pm 0.3) \%$. The result for the $e+$ jets channel is slightly higher than the $\mu+$ jets channel, but within statistical uncertainty. The measured asymmetry decreases as the number of jets increases, but within the statistical uncertainty.

\subsubsection{Reconstruction-level asymmetry dependencies}

In addition to measuring the reconstruction $\Delta y$-based asymmetries for all events, also known as the inclusive asymmetry, we measured the asymmetry for different ranges of $m_{t \bar{t}}$, the invariant mass of the $t \bar{t}$ system, and for different ranges of $|\Delta y|$. The specifics of these two differential measurements are driven in part by the dependence of the $A_{\mathrm{FB}}$ on $m_{t \bar{t}}$ seen by the CDF collaboration [33]. Two differential measurements are made: one measurement of $A_{\mathrm{FB}}$ for events with $m_{t \bar{t}}<450$ $\mathrm{GeV}$ and $m_{t \bar{t}}>450 \mathrm{GeV}$, and another measurement for events with $|\Delta y|<1$ and $|\Delta y|>1$. The results of the differential reconstruction-level asymmetry measurements are summarized in Figure 7.5. We do not see the overall dependence seen in the data of the CDF collaboration, but DØ data are consistent with the CDF result for each range of individual masses.
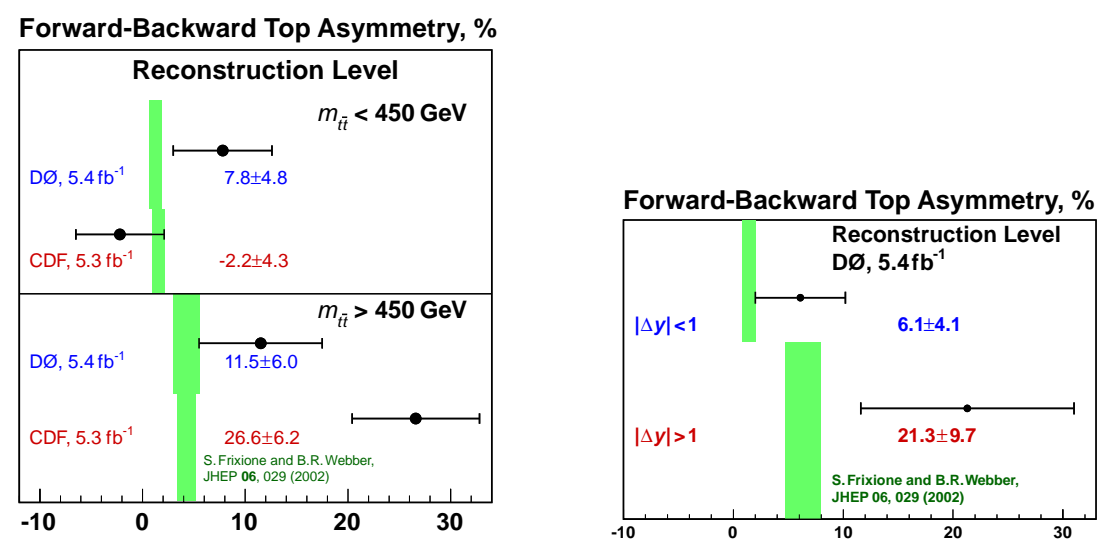

Figure 7.5 Differential asymmetry measurements with respect to $m_{t \bar{t}}$ (left) and $|\Delta y|$ (right). Results from the CDF collaboration are included in the left plot. 


\subsection{Production-level measurements}

In this section, the production-level measurements are explained in detail. To make a measurement at the production level, we use a process known as unfolding. Reference [103] explains unfolding in detail under the title of deconvolution. Unfolding parameterizes the simulated effects of reconstruction and acceptance and reverses them. For the unfolding techniques described below, the input $\Delta y$ and $q_{l} y_{l}$ distributions are from data after background subtraction.

Unfolding data is more complex than measuring the asymmetry in the detector after background subtraction, yet allows for more direct comparison with theory and other experiments. For instance, the reconstruction-level predictions are derived from $t \bar{t}$ events simulated with MC@NLO processed by the simulation of the $\mathrm{D} \varnothing$ detector. But more recent predictions go beyond QCD to include QED effects [39], which are presently not included in MC@NLO. Therefore we unfold the asymmetries to allow comparison to the latest theoretical work.

A proper understanding of the detector plays a vital role for both the reconstructionand production-level measurements. If the detector simulation does not match the behavior the actual detector and introduces (or fails to introduce) an asymmetry that is not in the real detector, comparisons to theory cannot be made. For the reconstruction-level measurement, the asymmetry from background-subtracted data would not be affected by improper simulation of the detector, but the predictions from MC@NLO after the detector simulation would be affected. Conversely, theoretical predictions are fine if the detector is improperly modeled, but the unfolded asymmetry would be affected.

\subsubsection{Unfolding the $\Delta y$ distribution in four regions using matrix inversion}

The method discussed in this subsection is based on one of the unfolding methods used by the CDF collaboration [33]. The basic idea is to take four different ranges in $\Delta y$ for both reconstructed values and generated values and create a matrix of the probabilities of an event generated within a given $\Delta y$ range to be reconstructed in another $\Delta y$ range. 
The following equation is the basis for matrix-based unfolding:

$$
\vec{N}_{\text {reco }}=\mathbf{S A} \vec{N}_{\text {prod }}
$$

In Equation 7.18, $\vec{N}_{\text {reco }}$ and $\vec{N}_{\text {prod }}$ are lists of $n$-dimensional vectors, where $n$ is the number of regions being unfolded. These vectors constitute the bin contents of the $\Delta y$ histogram of data after background subtraction. Following the CDF methodology, we use the following four $\Delta y$ ranges: $\Delta y<-1 ;-1<\Delta y<0 ; 0<$ $\Delta y<1$ and $1<\Delta y$.

Two matrices, $\mathbf{S}$ and $\mathbf{A}$ link $\vec{N}_{\text {reco }}$ and $\vec{N}_{\text {prod }}$ together. The $\mathbf{S}$ matrix is known as the migration matrix and gives the probability of an event generated with a $\Delta y$ value in bin $i$ to be reconstructed with a $\Delta y$ value in bin $j$. The A matrix is the selection efficiency in each region of produced $\Delta y$. Different regions have different selection efficiencies due to the intrinsic capabilities of the detector and the selection requirements. Figure 7.6 shows the $\mathbf{S}$ matrix and Table 7.4 shows the A matrix. Using a bit of linear algebra, the unfolding technique via matrix inversion is derived:

$$
\mathbf{A}^{-1} \mathbf{S}^{-1} \mathbf{S A} \vec{N}_{\text {prod }}=\vec{N}_{\text {prod }}=\mathbf{A}^{-1} \mathbf{S}^{-1} \vec{N}_{\text {reco }}
$$

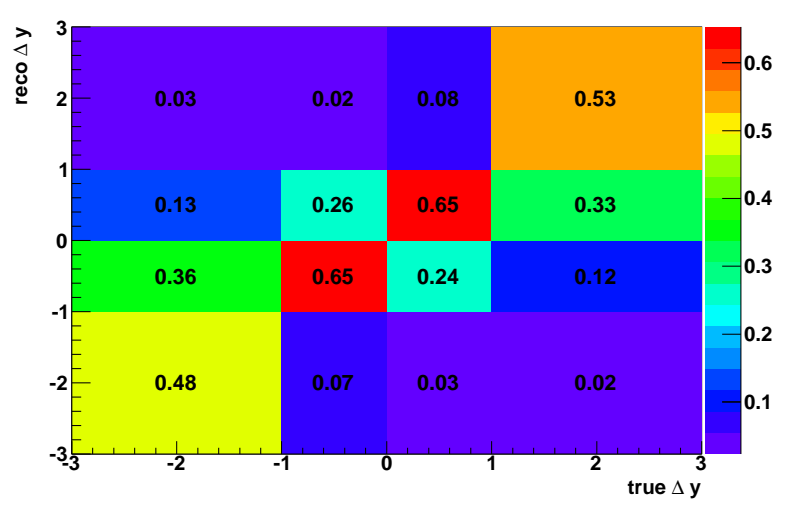

Figure 7.6 The 4 by 4 migration matrix used in the simplest form of unfolding.

For the background subtracted data, $\vec{N}_{\text {reco }}=(95,403,454,144)$. Solving Equation 7.19 gives $\vec{N}_{\text {prod }}=(100 \pm 28,354 \pm 53,452 \pm 55,187 \pm 31)$, with statistical 


$$
\mathrm{A}=\left[\begin{array}{cccc}
1.05 & 0 & 0 & 0 \\
0 & 0.998 & 0 & 0 \\
0 & 0 & 0.981 & 0 \\
0 & 0 & 0 & 0.970
\end{array}\right]
$$

Table 7.4 Relative selection efficiency between bins in $\Delta y$, shown so that the trace of $\mathrm{A}$ is equal to $4 . \Delta y$ increases as the entries go from left to right.

errors included. With only the effects of reconstruction included, $\mathrm{S}^{-1} \vec{N}_{\text {reco }}=$ $(104 \pm 27,367 \pm 52,451 \pm 53,173 \pm 29)$. The "MC@NLO corrected" asymmetries are $(16.9 \pm 8.0) \%$ with the acceptance matrix included. The uncertainty is calculated with Equation 2.11. The unfolded differential asymmetries with respect to $|\Delta y|$ are $12 \%$ for $|\Delta y|<1$ and $30 \%$ for $|\Delta y|>1$.

For completeness, we also simultaneously unfold $\Delta y$ and $m_{t \bar{t}}$ using the same technique as the CDF collaboration [33]. We chose not to publish this result because we didn't fully calculate the systematics and test the method. Also, the result is not particularly interesting as the reconstruction-level differential measurements already tell the story.

Here is a condensed description of how to use matrix inversion to simultaneously correct $\Delta y$ and $m_{t \bar{t}}$. Four different ranges are used:

$1 \Delta y<0$ and $m_{t \bar{t}}<450 \mathrm{GeV}$

$2 \Delta y>0$ and $m_{t \bar{t}}<450 \mathrm{GeV}$

$3 \Delta y<0$ and $m_{t \bar{t}}>450 \mathrm{GeV}$

$4 \Delta y>0$ and $m_{t \bar{t}}>450 \mathrm{GeV}$

The migration matrix shown in Figure 7.7 is the $\mathbf{S}$ matrix and the diagonal entries of $\mathbf{A} \approx 0.067,0.065,0.086$ and 0.076 . The results of this procedure are shown in Table 7.5. Using the CDF unfolding procedure on our data, with no further study, gives $A_{\mathrm{FB}}=20 \%$ for $m_{t \bar{t}}<450 \mathrm{GeV}$ and $A_{\mathrm{FB}}=18 \%$ for $m_{t \bar{t}}>450$ $\mathrm{GeV}$. 


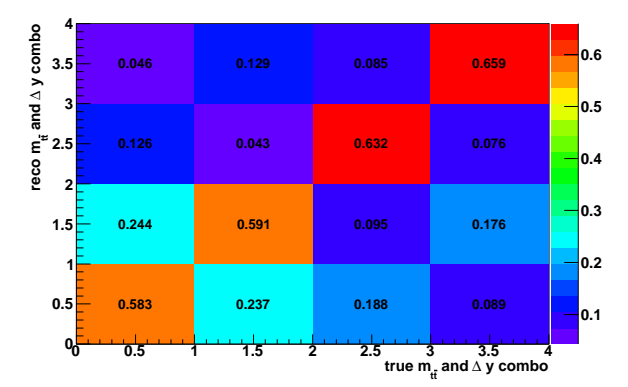

Figure 7.7 Migration matrix for different $\Delta y$ and $m_{t \bar{t}}$ regions.

\begin{tabular}{|l|c|c|}
\hline Type & $A_{\mathrm{FB}}(\%)\left(m_{t \bar{t}}<450 \mathrm{GeV}\right)$ & $A_{\mathrm{FB}}(\%)\left(m_{t \bar{t}}>450 \mathrm{GeV}\right)$ \\
\hline Raw data & $6.3 \pm 3.1$ & $9.5 \pm 4.3$ \\
CDF raw data & $-1.6 \pm 3.4$ & $21.0 \pm 4.9$ \\
BG sub data & $7.8 \pm 3.7$ & $11.5 \pm 5.0$ \\
Unfold (S ${ }^{-1}$ only) & 18.4 & 12.2 \\
Unfold & 19.9 & 17.7 \\
CDF Unfold & -11.6 & 47.5 \\
\hline
\end{tabular}

Table 7.5 Results using simultaneous unfolding of $\Delta y$ and $m_{t \bar{t}}$ with comparison of CDF results from Ref. [33]. 


\subsubsection{Unfolding the $\Delta y$ distribution using a maximum likelihood fit}

The technique presented here is analogous to unfolding by matrix inversion. Rather than explicitly making the migration matrix, we use a maximum likelihood fit similar to the one found in Section 7.2.

The general procedure for template-based unfolding goes as follows. First consider $\mathrm{M}$ regions in $\Delta y$. Construct templates with the discriminant, to separate signal from background, and with the reconstructed fractions $\frac{d N}{d(\Delta y)}$ in M bins of $\Delta y$, to do the unfolding. $\mathrm{M}+2$ different templates are fit to the distribution from data: $M$ templates for signal in each region of generated $\Delta y, W+$ jets, with $\frac{d N}{d(\Delta y)}$ taken from simulation and MJ, with $\frac{d N}{d(\Delta y)}$ taken from the L-T sample. This is essentially the same procedure as used in Section 7.2 , except $\frac{d N}{d(\Delta y)}$ replaces the sign of $\Delta y$.

The maximum likelihood fit uses the same log likelihood found in Equation 7.14, except that the $\mu_{i}$ term is replaced with Equation 7.20. The terms in Equation 7.20 are the same as in Equation 7.16, except that the terms $F_{1}$ through $\mathrm{F}_{n}$ are added. The terms $\mathrm{F}_{i}$ are the fractions of events in different ranges of generated $\Delta y$, the unfolded results. Equation 7.21 shows $\mu_{i}$ for the specific case of four different $\Delta y$ ranges.

$$
\begin{aligned}
\mu_{i}\left(N_{t}^{t \bar{t}}, N_{t}^{\mathrm{MJ}}, \mathrm{F}_{1}, \ldots, \mathrm{F}_{\mathrm{n}}\right) & =f_{i}^{t \bar{t}(\text { region } 1)} N_{t}^{t \bar{t}} \mathrm{~F}_{1}(1-\mathrm{C}) \\
& +\cdots \\
& +f_{i}^{t \bar{t}(\text { region } n-1)} N_{t}^{t \bar{t}} \mathrm{~F}_{\mathrm{n}-1}(1-\mathrm{C}) \\
& +f_{i}^{t \bar{t}(\text { region } n)} N_{t}^{t \bar{t}}\left(1-\sum_{i=1}^{n-1} \mathrm{~F}_{\mathrm{i}}\right)(1-C) \\
& +f_{i}^{W+\text { jets }}\left(N_{t}-N_{t}^{t \bar{t}}-N_{t}^{\mathrm{MJ}}\right)(1-C) \\
& +f_{i}^{l-t}\left(N_{t}^{\mathrm{MJ}}+C\left(N_{t}-N_{t}^{\mathrm{MJ}}\right)\right)
\end{aligned}
$$




$$
\begin{aligned}
\mu_{i}\left(N_{t}^{t \bar{t}}, N_{t}^{\mathrm{MJ}}, \mathrm{F}_{1}, \mathrm{~F}_{2}, \mathrm{~F}_{3}, \mathrm{~F}_{4}\right) & =f_{i}^{t \bar{t}(\text { region } 1)} N_{t}^{t \bar{t}} \mathrm{~F}_{1}(1-\mathrm{C}) \\
& +f_{i}^{t \bar{t}(\text { region } 2)} N_{t}^{t \bar{t}} \mathrm{~F}_{2}(1-\mathrm{C}) \\
& +f_{i}^{t \bar{t}(\text { region } 3)} N_{t}^{t \bar{t}} \mathrm{~F}_{3}(1-\mathrm{C}) \\
& +f_{i}^{t \bar{t}(\text { region } 4)} N_{t}^{t \bar{t}}\left(1-\mathrm{F}_{1}-\mathrm{F}_{2}-\mathrm{F}_{3}\right)(1-C) \\
& +f_{i}^{W+\text { jets }}\left(N_{t}-N_{t}^{t \bar{t}}-N_{t}^{\mathrm{MJ}}\right)(1-C) \\
& +f_{i}^{l-t}\left(N_{t}^{\mathrm{MJ}}+C\left(N_{t}-N_{t}^{\mathrm{MJ}}\right)\right)
\end{aligned}
$$

The results of template-based unfolding procedure are shown in Figure 7.8. The unfolded numbers of events are $(100,368,457,167)$, very similar to $\mathbf{S}^{-1} \vec{N}_{\text {reco }}$ from Section 7.3.1. When backgrounds are not included in the unfolding procedure, these numbers become exactly the same. Which means that unfolding via matrix inversion and unfolding via maximum likelihood fit are exactly the same. I will prove this equality the following way. First, the $i$ th component of the reconstruction-level vector $\vec{\mu}$ is

$$
\mu_{i}=\sum_{j} a_{i j} f_{j}
$$

where $a_{i j}$ is the migration matrix entry and $f_{j}$ is the $j$-th component of the production-level vector $\vec{f}$. Plugging in $\mu_{i}$ into the log likelihood from Equation 7.14 gives

$$
\log L=\sum_{i} n_{i} \log \mu_{i}-\mu_{i}=\sum_{i} n_{i} \log \left(\sum_{j} a_{i j} f_{j}\right)-\sum_{j} a_{i j} f_{j}
$$

The likelihood will be maximum when $\left.\frac{d(\log L)}{d f_{i}}\right|_{\vec{f}=\hat{f}}=0$ for each $f_{i}$. Taking the derivate, using $\frac{d f_{j}}{d f_{k}}=\delta_{j k}$ gives

$$
\begin{aligned}
\frac{d(\log L)}{d f_{k}} & =\sum_{i} \frac{n_{i}}{\sum_{j} a_{i j} \hat{f}_{j}} a_{i j} \delta_{j k}-a_{i j} \delta_{j k}=\sum_{i} \frac{n_{i}}{\sum_{j} a_{i j} \hat{f}_{j}} a_{i k}-a_{i k} \\
& =\sum_{i} \frac{\left(n_{i}-\sum_{j} a_{i j} \hat{f}_{j}\right) a_{i k}}{\sum_{j} a_{i j} \hat{f}_{j}}
\end{aligned}
$$


Equation 7.24 can only equal 0 for every $k$ if $n_{i}-\sum_{j} a_{i j} \hat{f}_{j}=0$. But this term is equivalent to $\vec{N}_{\text {reco }}=\mathbf{S} \vec{N}_{\text {prod }}$, which is Equation 7.19 without the acceptance matrix included. So matrix inversion unfolding and maximum likelihood unfolding are equivalent.

One advantage of unfolding via maximum likelihood fit over unfolding by matrix inversion is the correlations between the fitted backgrounds and the unfolded results are included by TMinuit, which makes the computation of uncertainties easier.
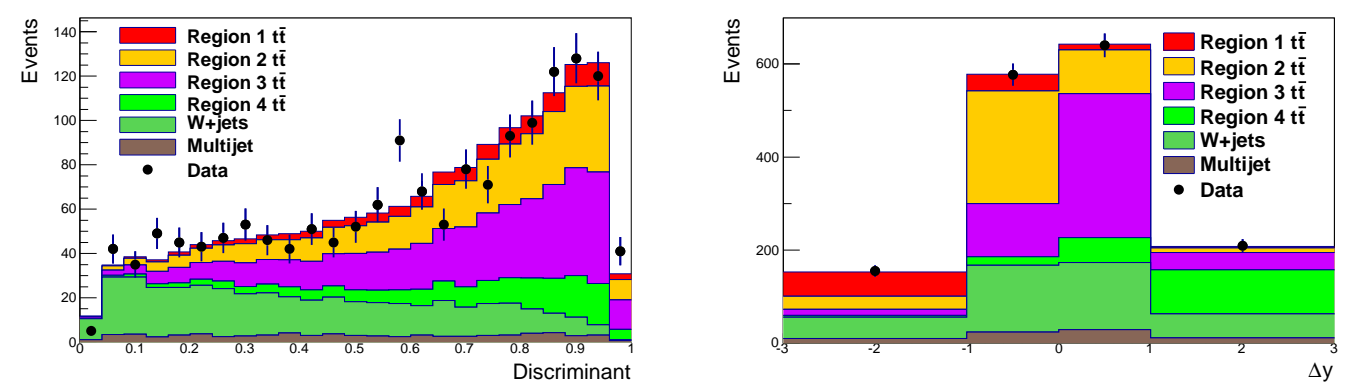

Figure 7.8 Data and simulation for the fit to the discriminant (left) and the fit to $\Delta y$ with the template unfolding technique (right).

\subsubsection{Issues with unfolding}

One of the more problematic parts of unfolding the asymmetry is accounting for the events near the $\Delta y=0$ boundary, which plays the special role of dividing forward and backward events. Imagine having a two-by-two migration matrix. On both sides, about $70 \%$ of the events produced with $\Delta y>0$ are reconstructed with $\Delta y>0$. But that is the average migration fraction. Events with produced with large $|\Delta y|$ migrate across 0 much less than $30 \%$ of the time, while events produced near zero may migrate with a rate of $50 \%$. So two bins are not enough to do a good job of describing the migrations. Each bin should be smaller than the resolution so that the migration fraction is constant for the entire bin, rather than an average, as shown in the above example.

Figure 7.9 shows the simulated resolution for three different observables used to measure angular properties of $t \bar{t}$ production: $\Delta y, q_{l} y_{l}$, and $y_{t, \text { had }}$, the rapidity 
of the top quark that decays entirely to jets, or the "hadronic" top, used by the CDF collaboration. Table 7.6 shows the RMS resolution of these three observables and the percentage of events within one standard deviation of zero, the dividing point between forward and backward. The observable with the worst resolution is $\Delta y$, which has long tails. The rapidity of the hadronic top quark is resolved two times better than $\Delta y$. The resolutions of both of these observables are almost 100 times worse when compared to the resolution of the rapidity of the lepton, which is essentially a delta function. For these reasons, we use $\Delta y$, which includes all of the information from the $t \bar{t}$ decay, and $q_{l} y_{l}$, which minimizes the effects of detection.

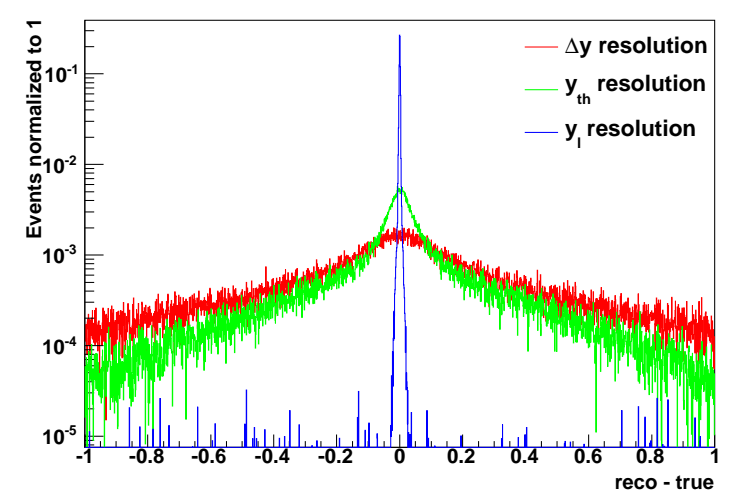

Figure 7.9 Experimental resolution for different observables. $y_{\text {th }}$ refers to the rapidity of the hadronically-decaying top quark. $y_{1}$ refers to the rapidity of the lepton.

Using many bins with pure matrix inversion causes large fluctuations, which is why wide bins are used in the methods described earlier. But Reference [103] warns: "Suppressing [fluctuations in unfolding via matrix inversion] by using wide bins can lead to inconsistent results."

\subsubsection{Regularized unfolding of the $\Delta y$ distribution}

The previous two unfolding techniques shown in Sections 7.3.1 and 7.3.2 are not the primary unfolding techniques used for this analysis. Rather, these techniques are used as crosschecks for the main unfolding technique, described here. As discussed in Section 7.3.3, a finely binned migration matrix is desirable. Each bin of the migration matrix from Section 7.3.1 is divided further into sixteen 


\begin{tabular}{|c|c|c|c|}
\hline Quantity & RMS & $1 \sigma$ from core & $\%$ of events within $1 \sigma$ of zero \\
\hline$\Delta y$ & 0.65 & 0.22 & $20 \%$ \\
$y_{t, \text { had }}$ & 0.37 & 0.08 & $10 \%$ \\
$q \cdot y_{l}$ in MC & 0.0023 & 0.0015 & $0.1 \%$ \\
$q \cdot y_{l}$ in cosmic data & - & 0.003 & $0.1 \%$ \\
\hline
\end{tabular}

Table 7.6 Experimental resolution and the fraction of problematic events for different observables. The cosmic ray resolutions in data from Ref. [106] are used as a crosscheck for the simulated resolution. $y_{t \text {,had }}$ refers to the rapidity of the hadronically-decaying top quark.

more bins. Due to low MC statistics, the outer eight bins $(-3<\Delta y<-2$ and $2<\Delta y<3)$ are merged into one bin. At the recommendation of Reference [103] and to better constrain the production-level $\Delta y$ distribution, more bins are used for reconstructed $\Delta y$ than for the production-level $\Delta y$. Except for the outermost bins, every two reconstruction-level bins of the migration matrix are merged into one production-level bin. The migration matrix has 50 bins for reconstructed $\Delta y$ and 26 bins for produced $\Delta y$, as shown in Figure 7.10. The contents of any bins with negative entries (due to limited statistics and negative weights from MC@NLO) are set to 0.
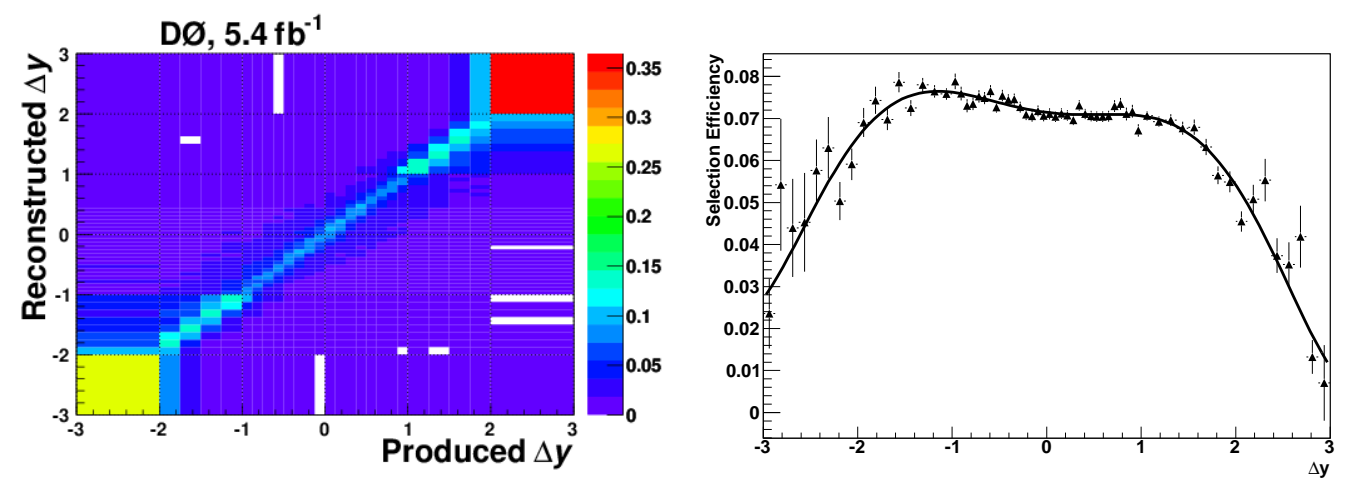

Figure 7.10 Left: Migration matrix used for regularization with 50 bins for reconstructed $\Delta y$ and 26 bins for the produced $\Delta y$. Right: Selection efficiency as a function of $\Delta y$.

Due to the small bin widths, large fluctuations will occur in the unfolded data sample. To control these fluctuations, an additional constraint is included, the assumption that the unfolded distribution is smooth. The process of constraining the unfolded distribution to be smooth is known as regularization. We use the 
TUnfold class $[107,108]$ included with ROOT to incorporate regularized unfolding into the measurement. Because the standard version of TUnfold does not allow for variable bin widths, we modified TUnfold accordingly.

To include regularization, the likelihood for the unfolded distribution of $\Delta y$ is modified such that:

$$
\log L(\vec{\theta})=\log L_{\text {stat }}(\vec{\theta})-\tau R_{\text {regu }}(\vec{\theta})
$$

where $L_{\text {stat }}$ is the likelihood term from the migration matrix, $\theta$ is the vector of bin contents of the produced $\Delta y$ histogram, $R_{\text {regu }}$ is the regularization condition and $\tau$ is the strength of the regularization [103]. For this analysis, we regularize on the curvature of the event density, where the event density is the contents of a bin divided by the bin width. This regularization is analogous to regularizing on the curvature of the bin density, a standard option in TUnfold, but extends the procedure to allow for variable binning.

When $\tau$ is zero, Equation 7.25 reduces to the usual maximum likelihood unfolding by matrix inversion. When $\tau \rightarrow \infty$, the regularization condition dominates. In the case of regularizing on curvature, the maximizing $\tau$ causes the unfolded distribution to be as linear as possible (minimizing curvature). There is a trade off in regularization for finely binned unfolding. Either there are rapid fluctuations in the unfolded distribution or there is a bias created, shaping the final distribution.

One way to optimize the $\tau$ parameter is known as the "L-curve" method [109]. Figure 7.11 shows an example L-curve. The L-curve shows the trade off between smoothness on the y-axis and distortion on the x-axis. The "kink" in the L-curve is considered to be the optimal point, where statistical fluctuations in the unfolded data are smoothed out, but the over all shape is not otherwise distorted. We use a slightly smaller regularization strength than suggested by the L-curve to further minimize the bias, as shown in Figure 7.13.

Figure 7.12 shows an example of a simulated data sample unfolded with different regularization strengths. In the left plot, the unfolding is under regularized and there are large fluctuations in the unfolded distribution. In the center plot, the regularization is near optimal and the unfolded $\Delta y$ distribution describes the production-level distribution fairly well. The right plot shows an over regularized distribution, which is flattened. 


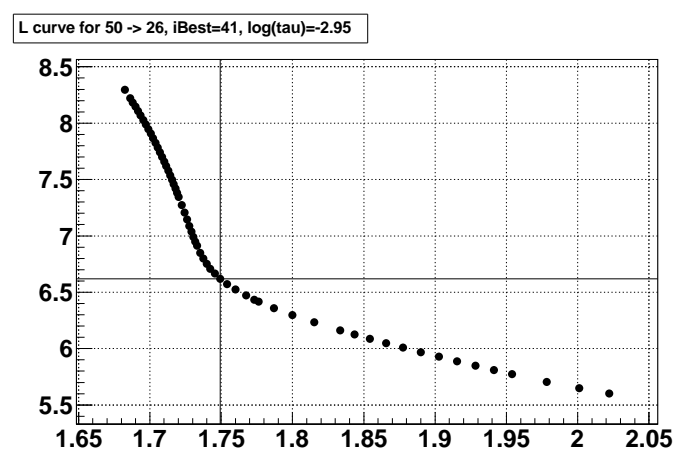

Figure 7.11 Example of an L-curve used to choose the regularization strength. The standard likelihood from matrix inversion is shown on the x-axis. The likelihood from the regularization term is shown on the y-axis. The size of the $\tau$ parameter and the amount of regularization increase as the points move toward the right of the plot.
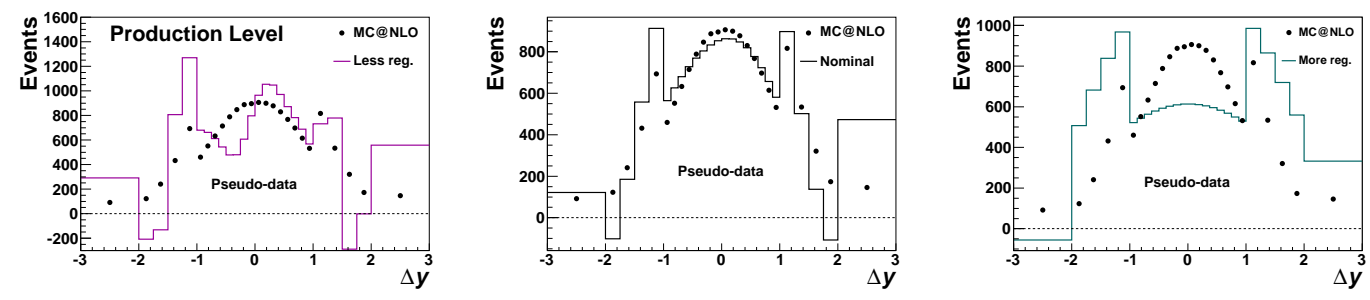

Figure 7.12 Example simulated data unfolded with different regularization strengths: under regularized (left), more-or-less optimally regularized (center) and over regularized (right).
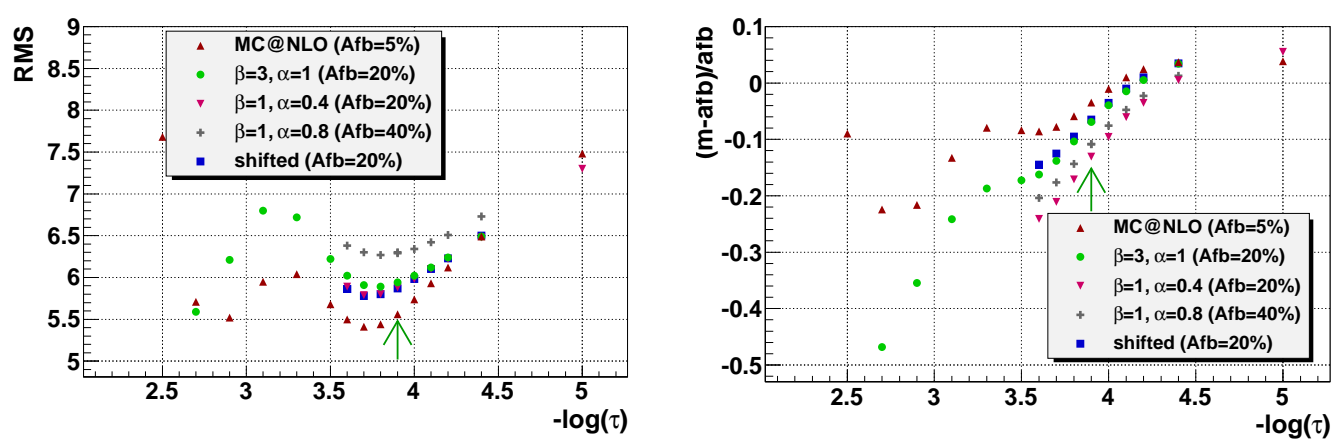

Figure 7.13 Effect of different regularization strengths on the statistical fluctuation and the bias of the asymmetry for multiple input distributions. The optimized parameter is shown with an arrow. 
Although bias to the unfolded asymmetry has been partially minimized by the choice of the regularization term, some residual effects remain. To understand the size of the bias, we used a series of pseudo experiments for many distributions of $\Delta y$, weighted to change $A_{\mathrm{FB}}$, and compared the output $A_{\mathrm{FB}}$ to the input $A_{\mathrm{FB}}$. More details about pseudo experiments, also known as ensemble testing, may be found in Section 7.4. The results of these tests are summarized in Figure 7.14. The bias factor is $0.93 \pm 0.05$, such that $A_{\mathrm{FB}}^{\text {unfolded }}=0.93 A_{\mathrm{FB}}^{\text {prod }}$.

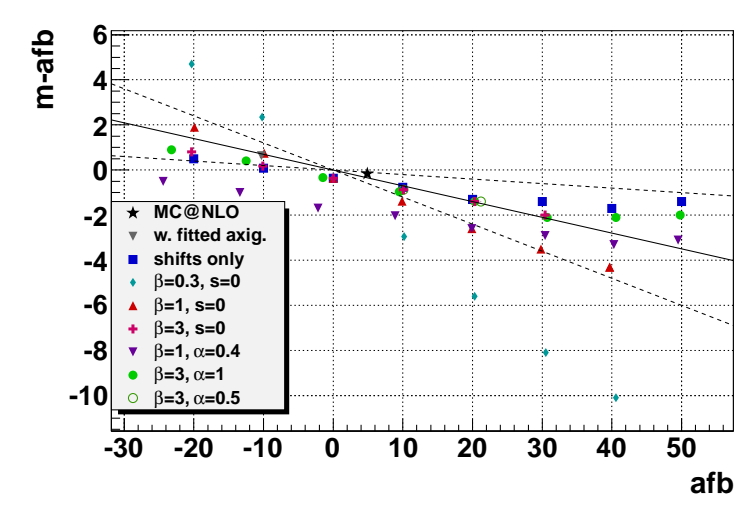

Figure 7.14 Bias for different $\Delta y$ distributions. For positive asymmetries, the bias in general causes the unfolded asymmetry to be smaller. The solid line is fit to the difference between the unfolded and true asymmetries. The dotted lines represent the systematic uncertainty on the bias correction.

To measure the asymmetry, the $\Delta y$ distribution from background-subtracted data is unfolded. Then the unfolded distribution is collapsed into the two asymmetry bins, $\Delta y>0$ and $\Delta y<0$, so the procedure is $50 \rightarrow 26 \rightarrow 2$. The asymmetry calculated from these two bins is corrected by dividing the result by the bias factor, leading to an unfolded asymmetry of $(19.6 \pm 6.0$ (stat) $) \%$. The difference between the unfolded measurement is more than two standard deviations larger the prediction from MC@NLO. The regularized $\Delta y$ distribution can be "folded" back with the migration matrix to give a comparison with data, as seen in Figure 7.15. 


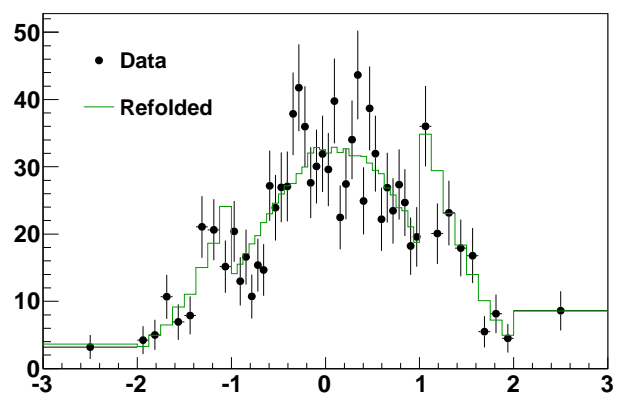

Figure 7.15 Comparison of $\Delta y$ distribution from data and the regularized $\Delta y$ distribution after "folding" it back to the reconstruction-level.

\subsubsection{Unfolding the $q_{l} y_{l}$ distribution}

Unfolding the $q_{l} y_{l}$ distribution is more straight forward than unfolding the $\Delta y$ distribution because migrations are much smaller. For this reason, only an acceptance correction is used. Using the same structure as the formulae shown earlier, unfolding the $q_{l} y_{l}$ distribution proceeds as following:

$$
\vec{N}_{\text {reco }}=\mathbf{S A} \vec{N}_{g e n}
$$

Since migrations are small, we approximate $\mathbf{S}$ as the identity matrix, $\mathbf{I}$, which means that $\mathbf{S A}=\mathbf{I A}=\mathbf{A}$, and leads to:

$$
\vec{N}_{\text {reco }}=\mathbf{A} \vec{N}_{\text {gen }}
$$

Unfolding becomes relatively simple:

$$
\vec{N}_{g e n}=\mathbf{A}^{-1} \vec{N}_{\text {reco }}
$$

Putting this equation in terms of a binned histogram, the unfolded result for the $i$ th bin becomes:

$$
\operatorname{bin}_{\text {gen }}^{i}=\frac{1}{\epsilon_{\text {sel }}^{i}} \operatorname{bin}_{\text {reco }}^{i}
$$

Figure 7.16 shows the selection efficiency for different $q_{l} y_{l}$ ranges. Notice the sharp change in selection efficiency at $\left|y_{l}\right|=1.5$. Because of this change, only 
events from with $\left|y_{l}<1.5\right|$ are included for the lepton-based measurements. The result from applying the acceptance correction to the $q_{l} y_{l}$ distribution is $A_{\mathrm{FB}}^{l}=15.2 \pm 3.8$ (stat). Table 7.7 shows the unfolded $A_{\mathrm{FB}}^{l}$ and the reconstructionlevel $A_{\mathrm{FB}}^{l}$ for comparison. Unfolding the lepton-based asymmetry for acceptance effects does not change the reconstruction asymmetry very much. The unfolded lepton-based asymmetry is more than three standard deviations from the MC@NLO prediction. The statistical uncertainty on the unfolded $A_{\mathrm{FB}}^{l}$ is evaluated with pseudo experiments (see Section 7.4).

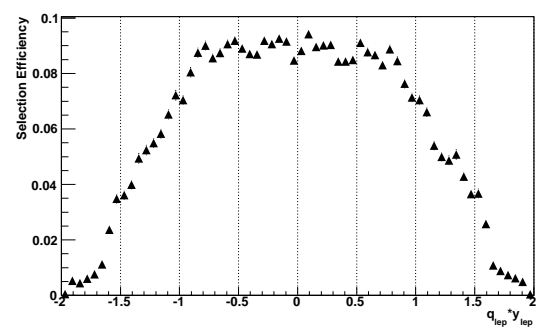

Figure 7.16 Selection efficiency as a function of $q_{l} y_{l}$. Because of the sharp drops at $\left|y_{l}\right|=1.5$, an additional selection criterion of $\left|y_{l}\right|<1.5$ is included for $A_{\mathrm{FB}}^{l}$.

\begin{tabular}{|l|c|c|}
\hline & \multicolumn{2}{|c|}{$A_{\mathrm{FB}}^{l}(\%)$} \\
& Data & $\mathrm{MC} @ \mathrm{NLO}$ \\
\hline Generated & & $2.1 \pm 0.1$ \\
Reconstructed & $14.2 \pm 3.6_{-0.8}^{+0.6}$ & $1.0 \pm 0.3 \pm 0.4$ \\
Unfolded & $15.2 \pm 3.8_{-1.3}^{+1.0}$ & $2.0 \pm 0.1$ \\
\hline
\end{tabular}

Table 7.7 Predicted and observed lepton-based asymmetries.

\subsection{Ensemble testing of procedures}

To understand the results of the four measurements and see how much simulated data might fluctuate from a central value, a procedure known as ensemble testing is used. In ensemble testing, groups of simulated events know as pseudo-data samples (PDSs) are put through the same analysis procedure as the real data sample collected by D $\varnothing$. 


\begin{tabular}{|l|c|}
\hline Physics process & Number of expected events \\
\hline$t \bar{t} l+$ jets & 1100 \\
$t \bar{t}$ dilepton & 32 \\
$W+$ jets & 333 \\
Diboson $(W W, W Z, Z Z)$ & 16 \\
Single top $(t b, t b q)$ & 12 \\
$Z+$ jets & 25 \\
Multijet & 80 \\
\hline
\end{tabular}

Table 7.8 Number of signal and background events for each type of process included in ensemble testing.

To make ensemble testing as close to reality as possible, all of the background processes are included, even though the measurement procedure still only fits for the $W+$ jets and multijet backgrounds. Since $\Delta y$ and $q_{l} y_{l}$ are correlated, the correlations are included for each input process. Poisson fluctuations in the number of signal and background events are also included, as well as the mild correlation between $\Delta y$ and the discriminant. The correlation between $q_{l} y_{l}$ and the discriminant is not explicitly included. The expected number of events are included in Table 7.8. These numbers are taken from yield tables for the dedicated $t \bar{t}$ cross section analysis, but using the same $b$-tagging criteria as this analysis. The number of $t \bar{t}$ events was adjusted to match the measured amount.

Different signal simulations with non-SM physics can also be included to compare to the data. Since actual $t \bar{t}$ events generated with BSM simulators do not currently exist with adequate statistics, events are weighted to emulate the asymmetric distributions based on the generated $\Delta y$ and $q_{l} y_{l}$ values. As shown in Equation 7.30, the production-level asymmetries $A_{\mathrm{FB}}$ and $A_{\mathrm{FB}}^{l}$ are weighted with multiple error functions to better match the asymmetry in data. Using the weights from Equation 7.30, the production level asymmetries become $A_{\mathrm{FB}}=20.7 \%$ and $A_{\mathrm{FB}}^{l}=15.5 \%$.

$$
w_{B S M}=1+0.55 \operatorname{Erf}\left(\frac{(\Delta y)_{g e n}}{3}\right)+0.2 \operatorname{Erf}\left(\frac{\left(q_{l} y_{l}\right)_{g e n}}{2}\right)
$$



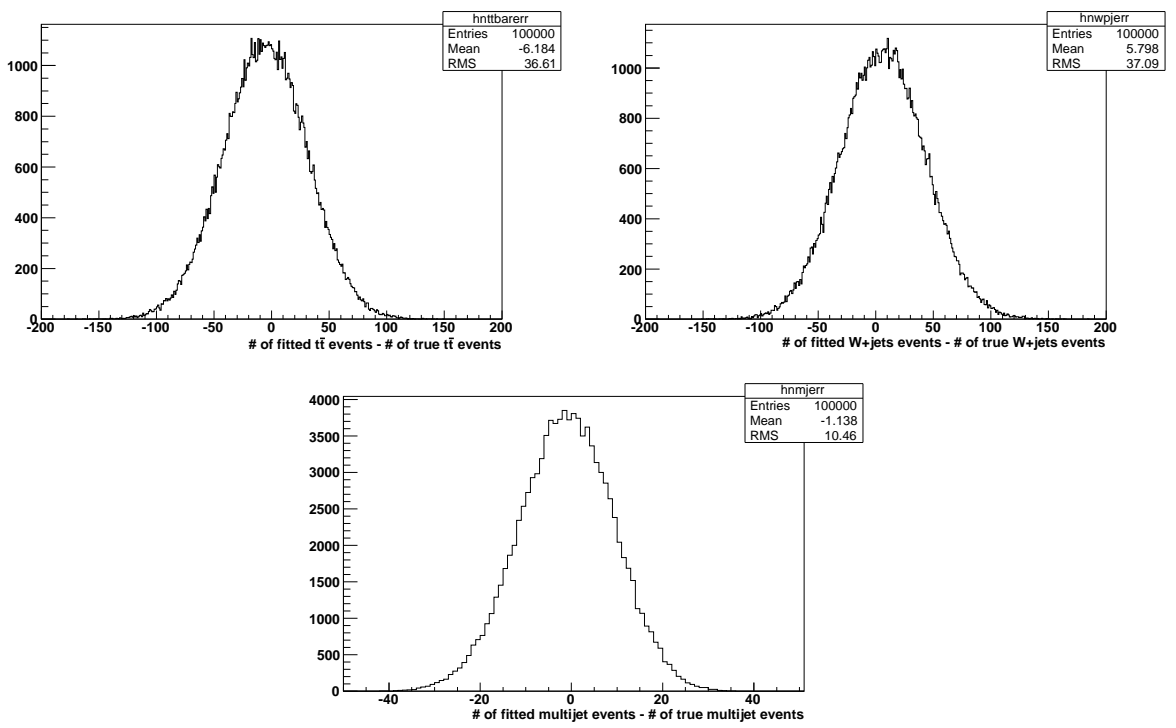

Figure 7.17 The fitted number of events minus the actual number of events in each PDS for $t \bar{t}$ (top, left), $W+$ jets (top, right) and MJ (bottom).

\subsubsection{Results of ensemble testing}

The figures shown in this section contain the main results for ensemble testing with 100,000 pseudo-data samples. The most important thing to test for is the ability of the template fit to properly measure the number of signal and background events. Figure 7.17 shows the difference between the fitted number of given type of event and the actual number of a given type event for each pseudo experiment. All pseudo events from $t \bar{t}$, including dilepton decays, are considered to be signal. Pseudo events from the L-T data sample are considered to be multijet (with the signal contamination factor included). All other pseudo events are considered to be $W+$ jets. There is very little bias due to these assumptions, as can be seen by the small mean of the histograms in Figure 7.17.

Another important thing is to check that the fitting procedure can correctly measure the true $t \bar{t}$ asymmetry in the pseudo data sample. As each pseudo data sample is artificial, the true asymmetries at the reconstruction level are known. The difference between the fitted reconstruction-level asymmetries and the true reconstruction-level asymmetries are measured in Figure 7.18. The resulting biases are about $-0.2 \%$ for $A_{\mathrm{FB}}$ and $-0.9 \%$ for $A_{\mathrm{FB}}^{l}$. Since the biases are negative, the 

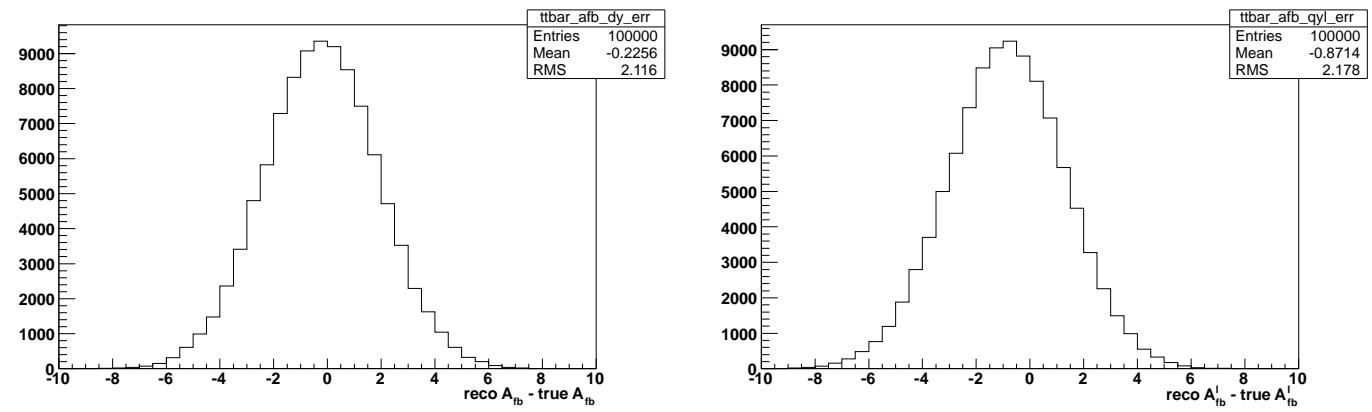

Figure 7.18 The fitted asymmetry minus the true asymmetry for each PDS for $A_{\mathrm{FB}}$ (left) and $A_{\mathrm{FB}}^{l}$ (right).

"actual" $t \bar{t}$ asymmetries being measured are larger. These biases makes sense, because only the asymmetry from the $W+$ jets background, which is larger than the other backgrounds, is included in the template, even though pseudo-events from $W+$ jets decays only account for about $80 \%$ of the events considered by the template fit to be $W+$ jets. These biases could be included as systematic uncertainties and would have a small overall effect on the uncertainty for the measurements. Since the predicted asymmetries do not include dilepton $t \bar{t}$ decays, the corresponding biases are not calculated. If events from dilepton $t \bar{t}$ decays are included as true signal events, the magnitudes of these biases are reduced by $0.15 \%$ to $-0.05 \%$ for $A_{\mathrm{FB}}$ and $-0.73 \%$ for $A_{\mathrm{FB}}^{l}$.

The interesting correlations between the different measurements are shown in Figure 7.19. Without ensemble testing there is no way to know how much the asymmetries are correlated. For instance, the unfolded $A_{\mathrm{FB}}$ and the reconstructed $A_{\mathrm{FB}}$ are about $75 \%$ correlated, which makes sense. A positive reconstructed $A_{\mathrm{FB}}$ is likely to lead to a positive unfolded $A_{\mathrm{FB}}$. The same connection is true for the reconstructed and unfolded lepton-based asymmetries, except more so, with a correlation factor of $97 \%$. The correlation between the observables $\Delta y$ and $q_{l} y_{l}$ is about 0.4 , which gives a ball park estimate for the correlation between $A_{\mathrm{FB}}$ and $A_{\mathrm{FB}}^{l}$. The bottom two scatter plots of Figure 7.19 show the correlations between the $A_{\mathrm{FB}}$ and $A_{\mathrm{FB}}^{l}$ for both reconstructed and unfolded quantities. Notice that the asymmetries are only about $25 \%$ correlated. We measure both $A_{\mathrm{FB}}$ and $A_{\mathrm{FB}}^{l}$, which are similar but almost independent of each other, giving the pair more strength. 

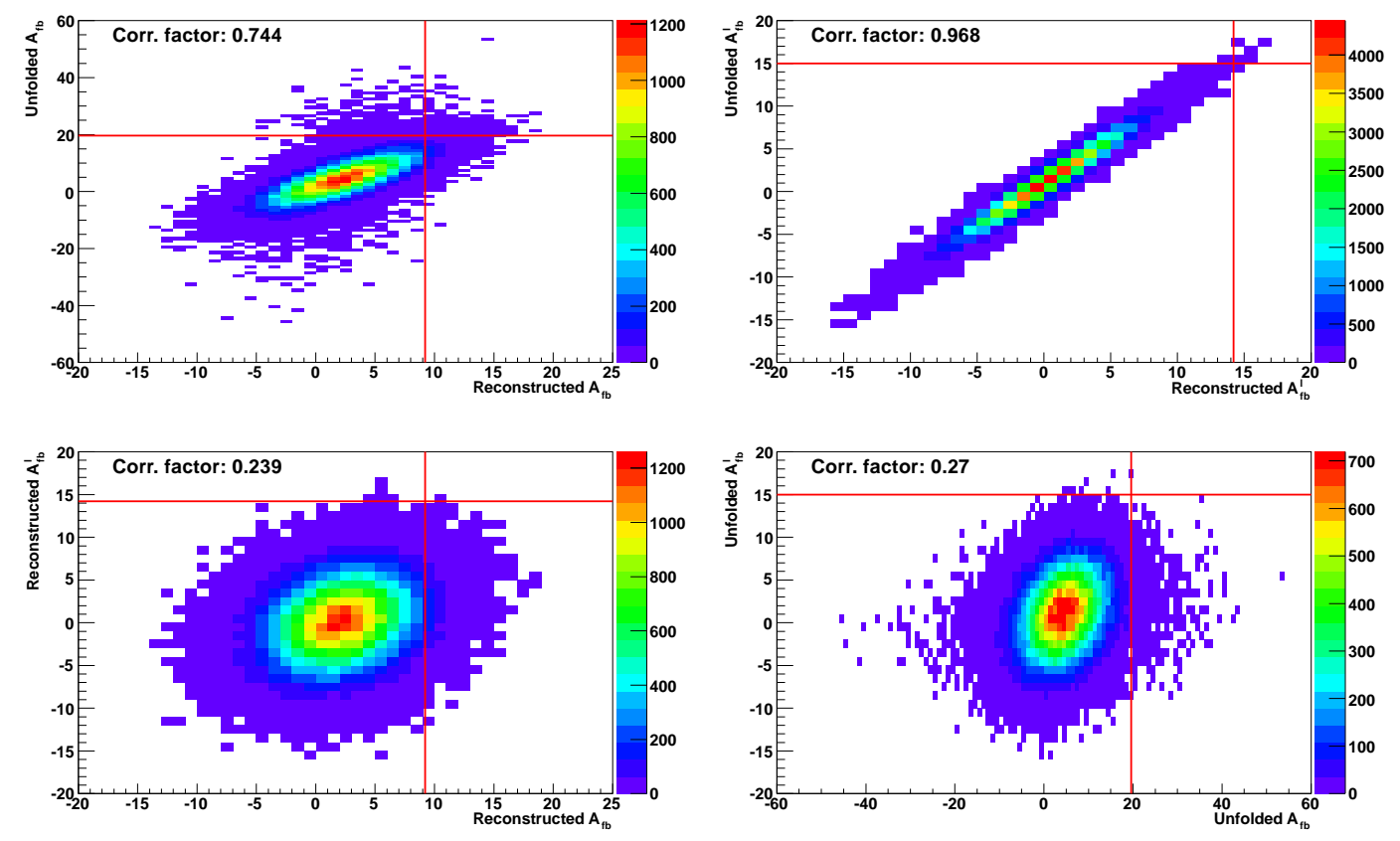

Figure 7.19 Correlation plots for unfolded $A_{\mathrm{FB}}$ versus reconstruction-level $A_{\mathrm{FB}}$ (top, left), unfolded $A_{\mathrm{FB}}^{l}$ versus reconstruction-level $A_{\mathrm{FB}}^{l}$ (top, right), reconstruction level $A_{\mathrm{FB}}^{l}$ versus reconstruction level $A_{\mathrm{FB}}$ (bottom, left) and unfolded $A_{\mathrm{FB}}^{l}$ versus unfolded $A_{\mathrm{FB}}$ (bottom, right). The red lines represent the values measured from data. 
In addition to scatter plots in Figure 7.20, contour bands are included that contain different " $\sigma$ " fractions of PDSs: $1 \sigma=68.3 \%, 2 \sigma=95.4 \%, 3 \sigma=99.7 \%$, $4 \sigma=99.99 \%$ and $5 \sigma=99.9999 \%$. Since $A_{\mathrm{FB}}$ and $A_{\mathrm{FB}}^{l}$ are normally distributed, the bivariate normal distribution is fit to the two dimensional scatter plot. The bivariate normal distribution has the following form:

$$
\frac{A}{2 \pi \sigma_{1} \sigma_{2} \sqrt{1-\rho^{2}}} e^{-\frac{1}{2} Z}
$$

where

$$
Z \equiv \frac{z_{1}^{2}+z_{2}^{2}-2 \rho z_{1} z_{2}}{1-\rho^{2}}
$$

Note that $z_{1} \equiv \frac{x_{1}-\mu_{1}}{\sigma_{1}}$ and $z_{2} \equiv \frac{x_{2}-\mu_{2}}{\sigma_{2}}$. In the bivariate normal distribution there are six free parameters: $A$ is the overall normalization factor, $\sigma_{1}$ and $\sigma_{2}$ are the variances of $x_{1}$ and $x_{2}, \mu_{1}$ and $\mu_{2}$ are the means of $x_{1}$ and $x_{2}$, and $\rho$ is the correlation factor. Contour lines are lines of equal height and, for the case of $A=1$, equal probability. When $A=1$, Equation 7.31 becomes a probability distribution function and contours occur when $Z=C$, some constant.

Now we need to know the area of the ellipse where $Z=C$. With help from References [110] and [111], one can see that $Z$ is equivalent to $\chi^{2}$ distribution with two degrees of freedom. Reference [111] states with proof, in murkier terms, that when $Z=\chi^{2}(\alpha)$, the area of the ellipse is $1-\alpha$. In this case, $\chi^{2}(\alpha)$ is $a$ in the $\int_{0}^{a} \chi^{2}=\alpha$, where the integral is the cumulative distribution function for $\chi^{2}$. The general cumulative distribution function for a $\chi^{2}$ distribution with $\mathrm{n}$ degrees of freedom is quite complicated, but in the case of two degrees of freedom, the cumulative distribution function is $1-e^{-\frac{\chi^{2}}{2}}=\alpha\left(\chi^{2}\right)$. Inverting the equation gives the value of $\chi^{2}$ for an ellipse with area $1-\alpha$ is $\chi^{2}(\alpha)=-2 \log (1-\alpha)$.

Substituting $Z=\chi^{2}(\alpha)=-2 \log (1-\alpha)$ into Equation 7.31 gives:

$$
\frac{1}{2 \pi \sigma_{1} \sigma_{2} \sqrt{1-\rho^{2}}} e^{-\frac{1}{2}(-2 \log (1-\alpha))}=\frac{1-\alpha}{2 \pi \sigma_{1} \sigma_{2} \sqrt{1-\rho^{2}}}
$$

Equation 7.33 gives the height of contour lines surrounding ellipses containing $\left(\alpha^{*} 100\right) \%$ of the distribution. The maximum height of this distribution is $\mathrm{h}(0)$ 
$=\frac{1}{2 \pi \sigma_{1} \sigma_{2} \sqrt{1-\rho^{2}}}$. Any other height can be expressed in terms of $\mathrm{h}(0): \mathrm{h}(\alpha)=$ $\mathrm{h}(0)(1-\alpha)$. So a contour with $90 \%$ of the maximum height contains $10 \%$ of the area and a contour with $10 \%$ of the maximum height contains $90 \%$ of the area. This expression is true even if the distribution is not normalized to 1.

To make contours for confidence intervals, the percentage of events within $n \sigma$ (for the 1D normal distribution) from the histogram maximum is $\alpha=\operatorname{Erf}\left(\frac{n}{\sqrt{2}}\right)$. The height of the $n$-th $\sigma$ confidence band becomes $\mathrm{h}(\mathrm{n})=\mathrm{h}(0)\left(1-\operatorname{Erf}\left(\frac{n}{\sqrt{2}}\right)\right)$. These bands are the contours that are drawn in Figure 7.20. In the top two plots, which use $t \bar{t}$ signal generated with MC@NLO, notice that the red star representing the measured values from data lies outside of the $3 \sigma$ contour lines. The $\rho$ values printed on the plots are in good agreement with the correlation factors taken directly from the histograms. In addition, the p-value can be measured from the contour that the measured values lie on. The p-value is the fraction of PDSs outside of the contour, measured by $\frac{h_{\text {data }}}{h_{\max }}$. For this analysis, the p-values are 0.00044 for the measured reconstruction-level asymmetries agreeing with the predictions from MC@NLO, and 0.00032 for the production-level asymmetries agreeing with the predictions from MC@NLO.

In the bottom two plots of Figure 7.20, the MC@NLO t $t \bar{t}$ signal is weighted such that the production-level asymmetries are $A_{\mathrm{FB}}=20.7 \%$ and of $A_{\mathrm{FB}}^{l}=15.5 \%$. Notice the variances are consistent with the unweighted signal. The correlation coefficients are also consistent, but this consistency is only due to the fact that $(\Delta y)_{g e n}$ and $\left(q_{l} y_{l}\right)_{g e n}$ are uncoupled in the weight and does not necessarily hold true. The measured mean unfolded values of $A_{\mathrm{FB}}=21.9 \%$ and $A_{\mathrm{FB}}^{l}=13.8 \%$ are off from the input values but within the systematic uncertainty for the calibration. As some values of the unfolded $A_{\mathrm{FB}}$ go outside of the $5 \sigma$ contour band, it seems to be the case that the distribution in the unfolded $A_{\mathrm{FB}}$ has non-Gaussian tails.

Another way to make the contour bands is to make ellipses with $\sigma_{x}$ and $\sigma_{y}$ for the semi-major and semi-minor axes, known as standard error bands [110, 103]. The probability for an event to lie within $n \sigma_{2 D}$ from the peak of a two-dimensional normal distribution is given by $1-e^{\frac{-n^{2}}{2}}$. The height of the $n$ th-sigma standard error band is $e^{\frac{-n^{2}}{2}}$. Figure 7.21 shows the reconstruction-level and production-level correlation plots with the standard error bands. 

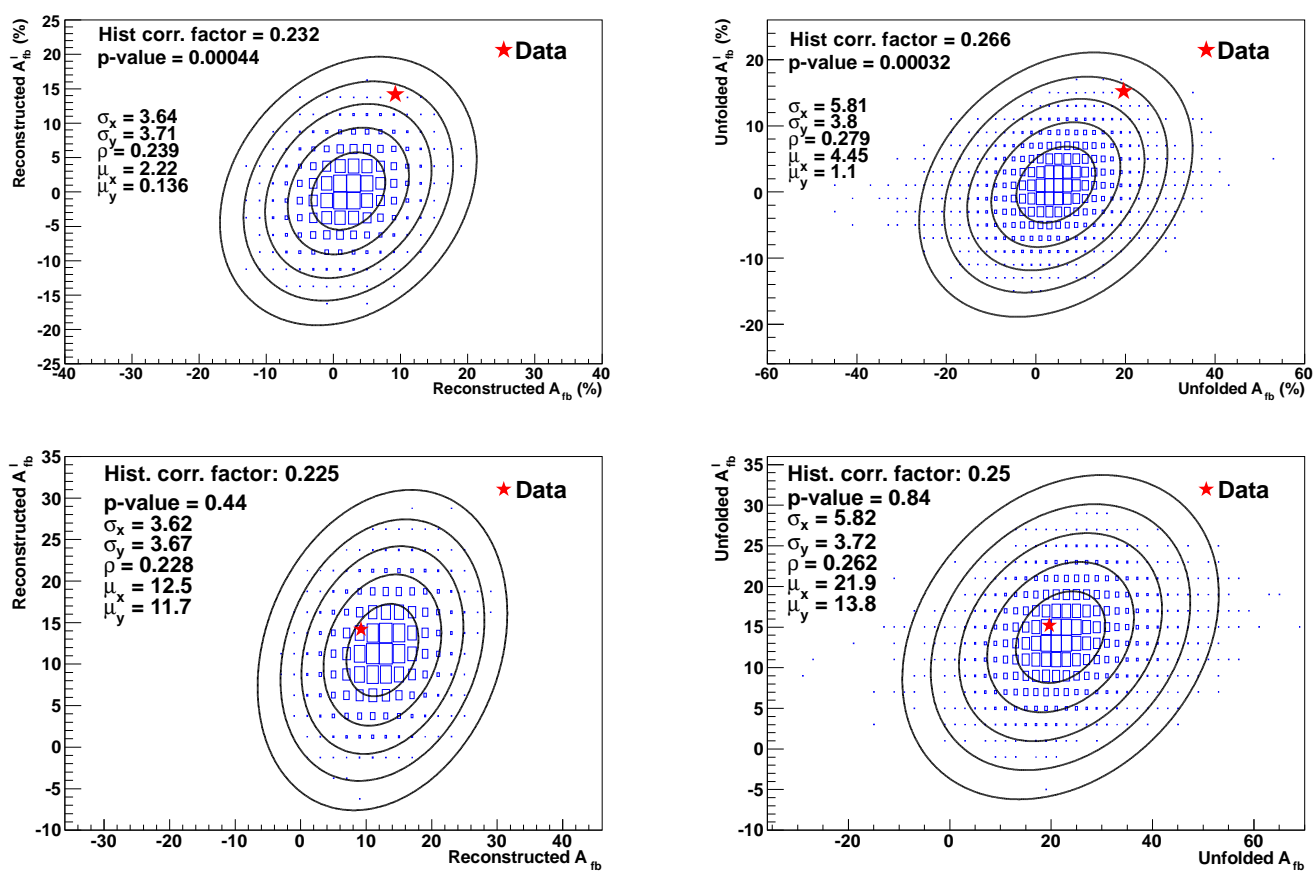

Figure 7.20 Correlation plots with confidence contour lines for different asymmetries. The plots on the left compare reconstruction-level asymmetries and the plots on the right compare unfolded asymmetries. Events in the bottom plots are weighted to change the production level asymmetries. The red point represents values measured from data. Systematic uncertainties are not included.
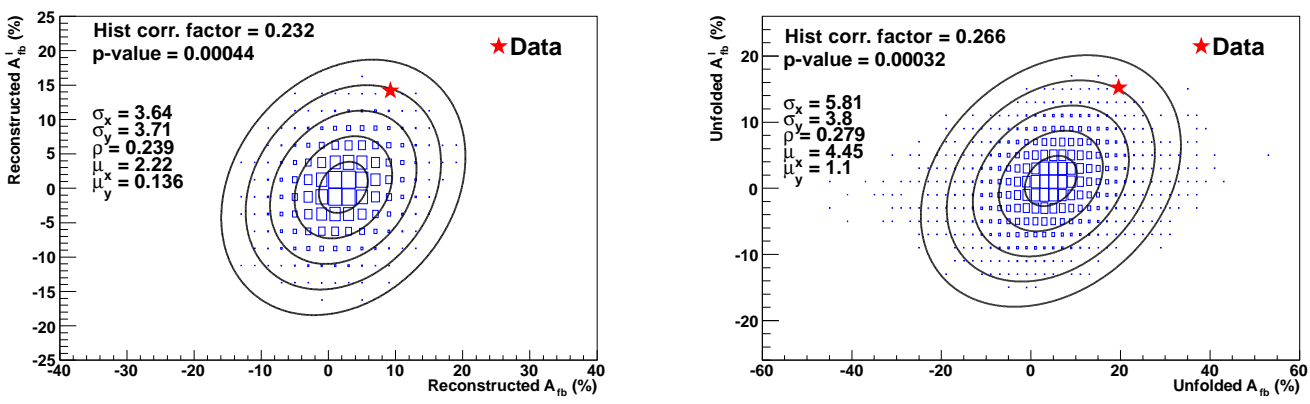

Figure 7.21 Correlation plots with standard error contour lines for different asymmetries. The plot on the left compares reconstruction-level asymmetries and the plot on the right compares unfolded asymmetries. The red point represents values measured from data. Systematic uncertainties are not included. 


\subsection{Crosschecks}

Multiple cross checks beyond ensemble testing are performed to make sure the inputs to the measurement make sense.

\subsubsection{Production cross section}

To make sure that the number of $t \bar{t}$ events fitted in the tight data sample is in agreement with other measurements, thus making sure that the template-based fitting procedure produces sensible results, we compute the $t \bar{t}$ production cross section for each channel. The cross section is found using the following equation:

$$
\sigma_{t \bar{t}}=\frac{N_{t \bar{t}}}{\epsilon_{S M} B(t \bar{t} \rightarrow l+\mathrm{jets}) \int L d t},
$$

where the branching fraction $B(t \bar{t} \rightarrow l+$ jets $)$ is $17.14 \%$ for the $e+$ jets channel and $17.21 \%$ for the $\mu+$ jets channel [16], $\int L d t$ is the integrated luminosity in each channel, and $\epsilon_{S M}$ is the selection efficiency.

- For Run IIa, the resulting cross sections are $8.2_{-0.9}^{+0.9}$ (stat) $\mathrm{pb}$ in the $e+$ jets channel, and $8.2_{-1.1}^{+1.1}$ (stat) pb in the $\mu+$ jets channel.

- For Run IIb, the resulting cross sections are $6.7_{-0.5}^{+0.5}$ (stat) $\mathrm{pb}$ in the $e+$ jets channel, and $8.7_{-0.6}^{+0.6}$ (stat) pb in the $\mu+$ jets channel.

The cross sections measured here are comparable with those found in the dedicated $l+$ jets cross section measurement using the same data sample [24]. That measurement used different event selection criteria, specifically a tighter $b$-tagging requirement as well as events with two and three jets. To compensate for the fact that $t \bar{t}$ events which decay via the dilepton channel yet pass the lepton+jets selection criteria were not included in the signal simulation, a factor of $\frac{1}{1.09}$ has been included.

\subsubsection{Directly measuring the asymmetry from $W+$ jets}

All of the techniques used in the measurement assume that the background distributions are well-modeled. The background distributions from multijet events are 
taken directly from data, so no dependence on simulation exists for the MJ background. On the other hand, the distributions for the $W+$ jets background, as well as $A_{\mathrm{FB}}$ and $A_{\mathrm{FB}}^{l}$ for $W+$ jets, are taken directly from ALPGEN+PYTHIA. Rather than take these predictions at face value, we designed the following technique to simultaneously measure the asymmetries from $W+$ jets as well as $t \bar{t}$ events. The asymmetry from the $W+$ jets background is measured with a template fitting technique similar to the template procedure described in Section 7.2.1. Equation 7.12 is modified such that:

$$
L\left(N_{t}^{t \bar{t}}, N_{t}^{\mathrm{MJ}}, A_{\mathrm{FB}}, W+j e t s A_{\mathrm{FB}}\right)=\left[\prod_{i} P\left(n_{i}^{o b s}, \mu_{i}\right)\right] \cdot P\left(N_{l-t}^{o b s}, N_{l-t}\right)
$$

The essential form of Equation 7.14 is used to make this new measurement, but the $\mu_{i}$ term is modified so that

$$
\begin{aligned}
\mu_{i}\left(N_{t}^{t \bar{t}}, N_{t}^{\mathrm{MJ}}, A_{\mathrm{FB}}, W+j e t s A_{\mathrm{FB}}\right) & =f_{i}^{t \bar{t}(\Delta y>0)} N_{t}^{t \bar{t}}\left(\frac{1+A_{\mathrm{FB}}}{2}\right)(1-C) \\
& +f_{i}^{t \bar{t}(\Delta y<0)} N_{t}^{t \bar{t}}\left(\frac{1-A_{\mathrm{FB}}^{f i t}}{2}\right)(1-C) \\
& +f_{i}^{W+j e t s}\left(N_{t}-N_{t}^{t \bar{t}}-N_{t}^{\mathrm{MJ}}\right) \\
& \cdot\left(\frac{1+W+j e t s A_{\mathrm{FB}}}{2}\right)(1-C) \\
& +f_{i}^{W+j e t s}\left(N_{t}-N_{t}^{t \bar{t}}-N_{t}^{\mathrm{MJ}}\right) \\
& \cdot\left(\frac{1-W+j e t s A_{\mathrm{FB}}}{2}\right)(1-C) \\
& +f_{i}^{l-t}\left(N_{t}^{\mathrm{MJ}}+C\left(N_{t}-N_{t}^{\mathrm{MJ}}\right)\right)
\end{aligned}
$$

In order to better constrain the equation, events without any $b$-tagged jets but passing all of the other selection criteria are included in the templates. Additionally, the templates are divided into three sections for events with zero $b$ tags, one $b$ tag and two or more $b$ tags, as seen in Figures 7.22 and 7.23. Since the fitted asymmetries now include events without any $b$-tagged jets, we checked to 
make sure that the simulated asymmetries did not vary much with respect to $b$ tagging. The maximum difference between predicted asymmetries is about $2 \%$ for events with different numbers of $b$ tags. Figures 7.24, 7.25 and 7.26 show the distributions in $\Delta y$ and $q_{l} y_{l}$ for events with 0,1 and $\geq 2 b$-tagged jets, respectively. Table 7.9 shows the measured $t \bar{t}$ asymmetries for events separated by the number of $b$-tagged jets.
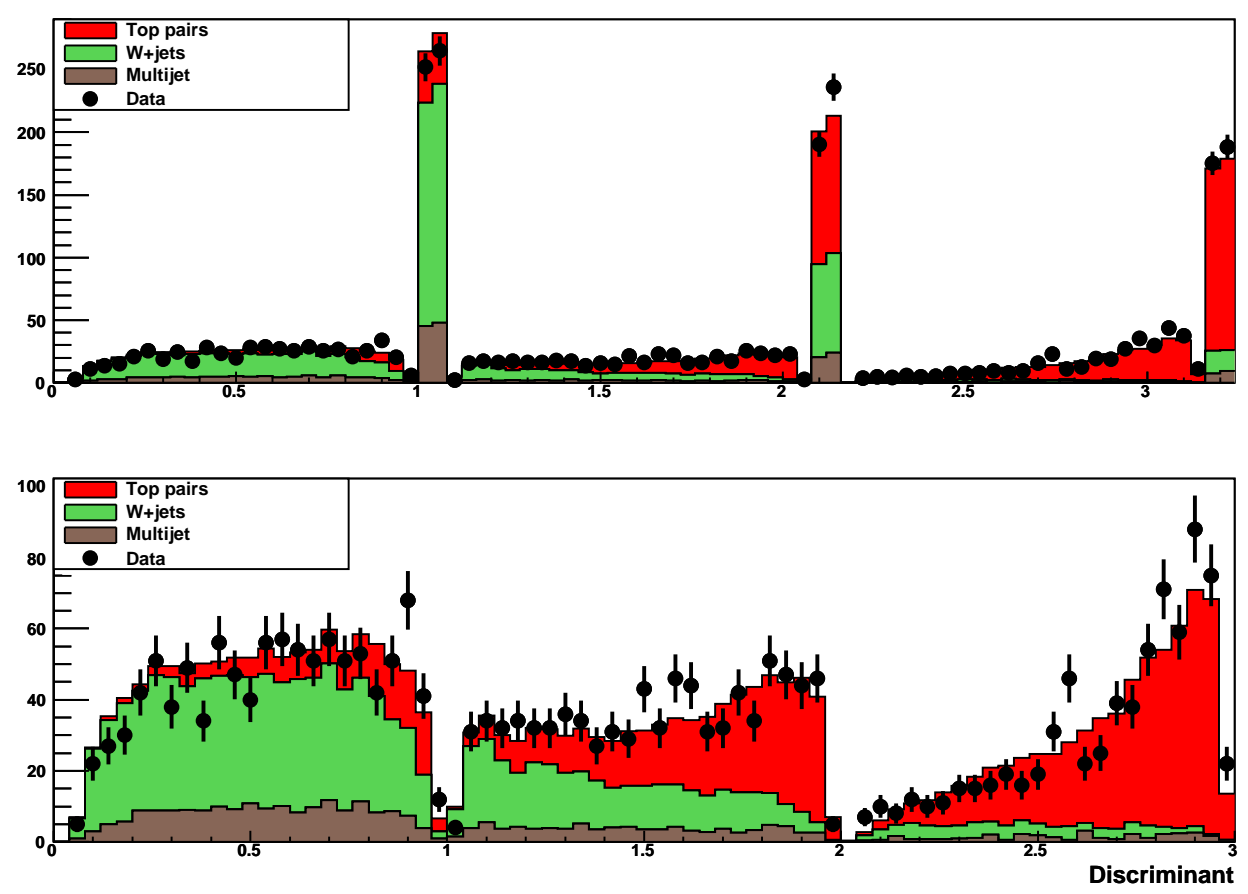

Figure 7.22 The templates used in the fit for the $W+$ jets $\Delta y$-based asymmetry. Shown fully (top), and without the $\operatorname{sgn}(\Delta y)$ bins below (bottom).

\begin{tabular}{|l|c|c|}
\hline Number of $b$-tagged jets & $A_{\mathrm{FB}}(\%)$ & $A_{\mathrm{FB}}^{l}(\%)$ \\
\hline 0 & $0 \pm 12$ & $9 \pm 12$ \\
1 & $17.0 \pm 6.7$ & $26.9 \pm 6.8$ \\
$\geq 2$ & $3.8 \pm 4.2$ & $5.5 \pm 4.3$ \\
\hline
\end{tabular}

Table 7.9 $A_{\mathrm{FB}}$ and $A_{\mathrm{FB}}^{l}$ shown for events with different numbers of $b$-tagged jets.

Results from the simultaneous template fit of $t \bar{t} A_{\mathrm{FB}}$ and $W+$ jets $A_{\mathrm{FB}}$ are shown in Table 7.10 , along with the $W+$ jets $A_{\mathrm{FB}}$ predictions from ALPGEN+PYTHIA. 

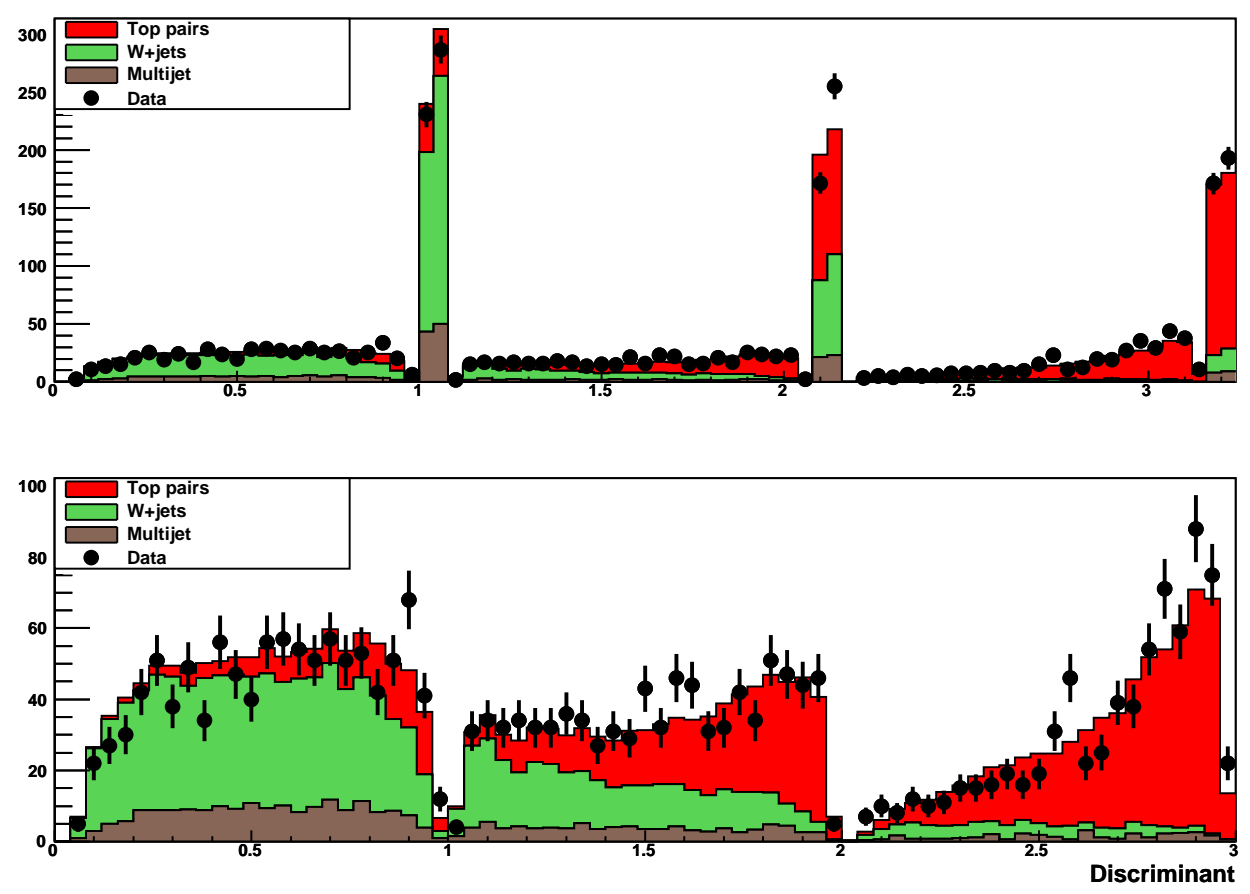

Figure 7.23 The templates used in the fit for the $W+$ jets $q_{l} y_{l}$-based asymmetry. Shown fully (top), and without the $\operatorname{sgn}\left(q_{l} y_{l}\right)$ bins (bottom).
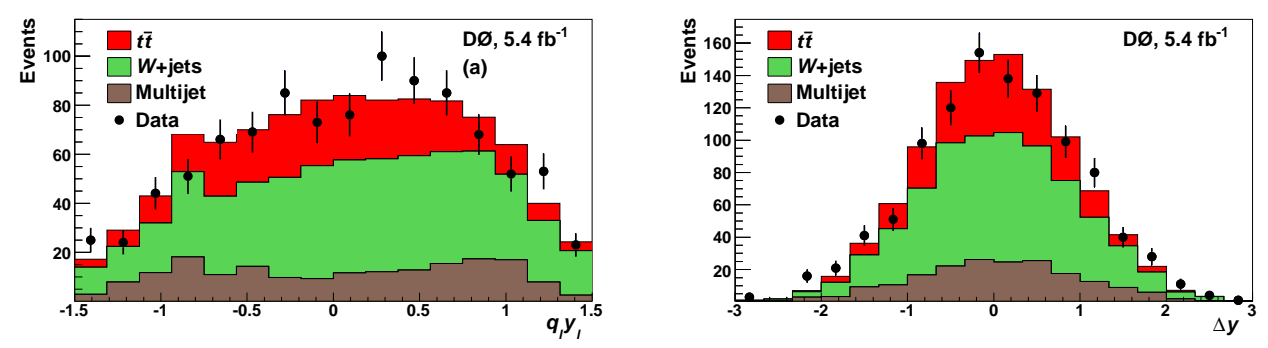

Figure 7.24 Data versus simulation for the $q_{l} y_{l}$ (left) and $\Delta y$ (right) distributions for events with $0 b$-tagged jets. 

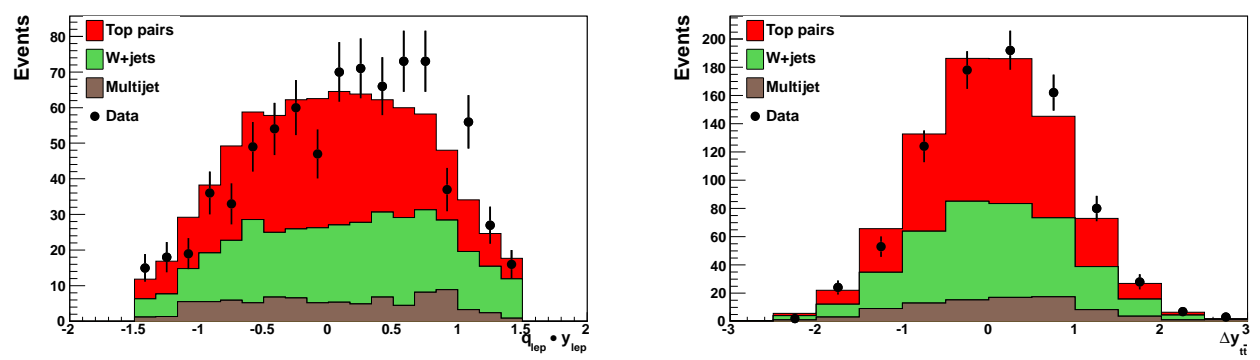

Figure 7.25 Data versus simulation for the $q_{l} y_{l}$ (left) and $\Delta y$ (right) distributions for events with $1 b$-tagged jet.
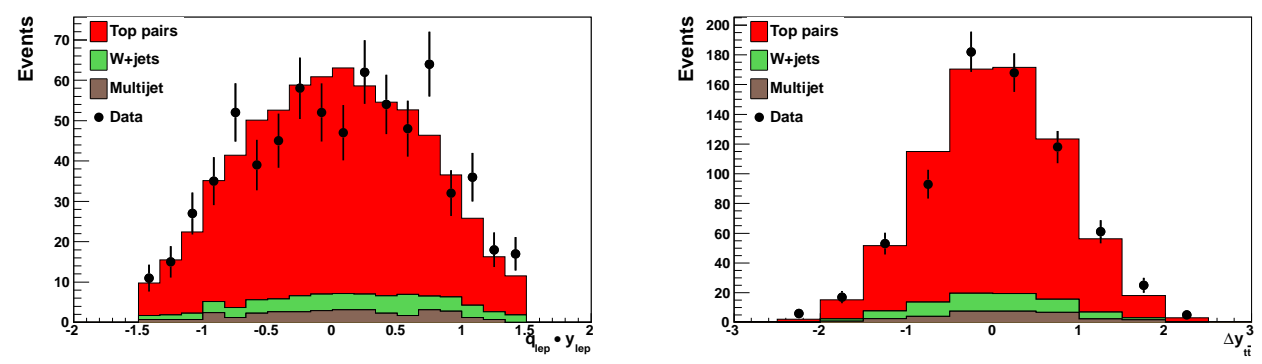

Figure 7.26 Data versus simulation for the $q_{l} y_{l}$ (left) and $\Delta y$ (right) distributions for events with 2 or more $b$-tagged jets. 
Similar results for the lepton-based $t \bar{t}$ and $W+$ jets asymmetries are shown in Table 7.11. As can be seen from the two tables, the predicted asymmetries for the $W+$ jets background by ALPGEN+PYTHIA are in good agreement with the asymmetries measured in data. Note that this crosscheck is also the first measurement of the asymmetry in $W+\geq 4$ jets.

\begin{tabular}{|l||c|c|c|c|c|}
\hline & Combined & $e+$ jets & $\mu+$ jets & 4 jets & $\geq 4$ jets \\
\hline$N_{\mathrm{t}}$ & 2615 & 1445 & 1170 & 2237 & 378 \\
$N_{\mathrm{l-t}}$ & 1808 & 1547 & 261 & 1550 & 258 \\
\hline$N_{t \bar{t}}$ & $1230 \pm 27$ & $621 \pm 19$ & $611 \pm 19$ & $990 \pm 25$ & $239 \pm 10$ \\
$N_{W}$ & $1150 \pm 27$ & $605 \pm 19$ & $550 \pm 19$ & $1044 \pm 25$ & $106 \pm 10$ \\
$N_{\mathrm{MJ}}$ & $235 \pm 7$ & $219 \pm 7$ & $9 \pm 3$ & $201 \pm 7$ & $33 \pm 3$ \\
$A_{\mathrm{FB}}(\%)$ & $7 \pm 4$ & $9 \pm 5$ & $5 \pm 6$ & $10 \pm 4$ & $-4 \pm 8$ \\
$W+$ jets $A_{\mathrm{FB}}(\%)$ & $4 \pm 4$ & $-2 \pm 5$ & $10 \pm 6$ & $3 \pm 4$ & $12 \pm 15$ \\
\hline Pred. $W A_{\mathrm{FB}}(\%)$ & $1.8 \pm 1.4$ & $-1.8 \pm 2.0$ & $6.4 \pm 1.9$ & $1.5 \pm 1.5$ & $4 \pm 5$ \\
\hline
\end{tabular}

Table 7.10 Fitted sample compositions and $\Delta y$-based asymmetries for $t \bar{t}$ and $W+$ jets, with the simulated $W+$ jets asymmetry.

\begin{tabular}{|l||c|c|c|c|c|}
\hline & Combined & $e+$ jets & $\mu+$ jets & 4 jets & $\geq 4$ jets \\
\hline$N_{t \bar{t}}$ & $1229 \pm 27$ & $620 \pm 19$ & $610 \pm 19$ & $992 \pm 25$ & $239 \pm 10$ \\
$N_{W}$ & $1151 \pm 27$ & $605 \pm 19$ & $550 \pm 19$ & $1044 \pm 25$ & $106 \pm 10$ \\
$N_{\mathrm{MJ}}^{l}(\%)$ & $235 \pm 7$ & $220 \pm 7$ & $10 \pm 3$ & $201 \pm 7$ & $33 \pm 3$ \\
$A_{\mathrm{FB}}^{l}(\%)$ & $11 \pm 4$ & $13 \pm 5$ & $8 \pm 6$ & $12 \pm 4$ & $6 \pm 8$ \\
$W+$ jets $A_{\mathrm{FB}}^{l}(\%)$ & $15 \pm 4$ & $14 \pm 5$ & $15 \pm 6$ & $16 \pm 4$ & $-2 \pm 15$ \\
\hline Pred. $W A_{\mathrm{FB}}^{l}(\%)$ & $14.3 \pm 1.4$ & $9.5 \pm 2.1$ & $20.5 \pm 1.8$ & $14.2 \pm 1.5$ & $16 \pm 5$ \\
\hline
\end{tabular}

Table 7.11 Fitted sample compositions and lepton-based asymmetries for $t \bar{t}$ and $W+$ jets, with the simulated $W+$ jets asymmetry. The number of data events $\left(N_{\mathrm{t}}\right.$ and $\left.N_{\mathrm{l}-\mathrm{t}}\right)$ is the same as in Table 7.10.

\subsubsection{Measurements with different magnetic field configu- rations}

An interesting crosscheck, also used in other D $\varnothing$ asymmetry measurements (such as Reference [112]), is to measure the asymmetry with different magnetic field configurations. Checking the asymmetry versus the magnetic field configuration 
is one way to test that the detector is not adding an inherent asymmetry to the data. Because there are two different magnet systems that have two different polarity settings in the $\mathrm{D} \varnothing$ detector, there are four combinations of magnet settings. Tables 7.12 and 7.13 show the measurements of $A_{\mathrm{FB}}$ and $A_{\mathrm{FB}}^{l}$ with the different magnet settings. Because $A_{\mathrm{FB}}^{l}$ depends completely on the measurement of the lepton kinematics, which depend on the magnetic field, this measurement should be most sensitive to the field configuration. Table 7.15 shows the dependence of the $A_{\mathrm{FB}}^{l}$ on the toroid polarity separately for $e+$ jets and $\mu+$ jets. A similar set of measurements of $A_{\mathrm{FB}}$ are shown in Table 7.14.

No significant dependencies on the magnet polarities are observed. In terms of the development of the analysis, the somewhat large change of $(23 \pm 12) \%$ in the asymmetry between the (tor +, sol -) and (tor -, sol -) magnet polarity settings helped to influence us to check the lepton-based asymmetry, which is more stable under changes in the magnetic field, and also much larger than we expected it to be. We conclude that no obvious detector biases are apparent in the data.

\begin{tabular}{|l||c|c|c|c|}
\hline & tor,+ sol + & tor,+ sol - & tor -, sol + & tor -, sol - \\
\hline$N_{\mathrm{t}}$ & 380 & 385 & 423 & 393 \\
$N_{\Delta y>0}$ & 188 & 200 & 234 & 227 \\
$N_{\Delta y<0}$ & 192 & 185 & 189 & 166 \\
$N_{\mathrm{l}-\mathrm{t}}$ & 178 & 182 & 167 & 189 \\
\hline Data $A_{\mathrm{FB}}(\%)$ & $-1 \pm 5$ & $4 \pm 5$ & $11 \pm 5$ & $16 \pm 5$ \\
$\mathrm{~L}-\mathrm{T} A_{\mathrm{FB}}(\%)$ & $-2 \pm 7$ & $13 \pm 7$ & $10 \pm 8$ & $13 \pm 7$ \\
\hline$N_{t \bar{t}}$ & $260 \pm 19$ & $284 \pm 19$ & $302 \pm 20$ & $268 \pm 20$ \\
$N_{W}$ & $100 \pm 19$ & $80 \pm 19$ & $104 \pm 20$ & $104 \pm 20$ \\
$N_{\mathrm{MJ}}$ & $20 \pm 2$ & $21 \pm 2$ & $17 \pm 2$ & $22 \pm 2$ \\
$A_{\mathrm{FB}}(\%)$ & $-2 \pm 8$ & $3 \pm 7$ & $14 \pm 7$ & $21 \pm 8$ \\
\hline
\end{tabular}

Table 7.12 Fitted sample compositions and $A_{\mathrm{FB}}$ by magnet polarity.

\subsubsection{Measurements by lepton charge}

To check whether the asymmetry measurements are dependent on the charge of the lepton, $A_{\mathrm{FB}}$ and $A_{\mathrm{FB}}^{l}$ are measured for events with $q_{l}>0$ and $q_{l}<0$. A difference would mean that top quarks and antiquarks behave differently, either from a violation of $\mathrm{CP}$ symmetry or from a mis-measurement by the detector. Detector 


\begin{tabular}{|l||c|c|c|c|}
\hline & tor,+ sol + & tor,+ sol - & tor -, sol + & tor -, sol - \\
\hline$N_{\Delta y>0}$ & 206 & 227 & 242 & 221 \\
$N_{\Delta y<0}$ & 174 & 158 & 181 & 172 \\
\hline Data $A_{\mathrm{FB}}^{l}(\%)$ & $8 \pm 5$ & $18 \pm 5$ & $14 \pm 5$ & $12 \pm 5$ \\
$\mathrm{~L}-\mathrm{T} A_{\mathrm{FB}}^{l}(\%)$ & $6 \pm 7$ & $-1 \pm 7$ & $2 \pm 8$ & $8 \pm 7$ \\
\hline$A_{\mathrm{FB}}^{l}(\%)$ & $7 \pm 8$ & $21 \pm 7$ & $16 \pm 7$ & $12 \pm 8$ \\
\hline
\end{tabular}

Table 7.13 Fitted sample compositions and $A_{\mathrm{FB}}^{l}$ by magnet polarity. The number of data events $\left(N_{\mathrm{t}}\right.$ and $\left.N_{\mathrm{lt}}\right)$ and the fitted sample composition $\left(N_{t \bar{t}}, N_{W}\right.$, and $\left.N_{\mathrm{MJ}}\right)$ are the same as in Table 7.10.

\begin{tabular}{|l||c|c|c|c|}
\hline & tor,$+ e+$ jets & tor,$- e+$ jets & tor,$+ \mu+$ jets & tor,$- \mu+$ jets \\
\hline$N_{\Delta y>0}$ & 461 & 177 & 211 & 244 \\
$N_{\Delta y<0}$ & 355 & 164 & 213 & 184 \\
\hline Data $A_{\mathrm{FB}}(\%)$ & $13 \pm 3$ & $4 \pm 5$ & $0 \pm 5$ & $14 \pm 5$ \\
$\mathrm{~L}-\mathrm{T} A_{\mathrm{FB}}(\%)$ & $12 \pm 5$ & $-4 \pm 12$ & $8 \pm 6$ & $11 \pm 6$ \\
\hline$A_{\mathrm{FB}}(\%)$ & $17 \pm 5$ & $5 \pm 7$ & $-3 \pm 8$ & $17 \pm 7$ \\
\hline
\end{tabular}

Table 7.14 $A_{\mathrm{FB}}$ by toroid polarity and channel.

\begin{tabular}{|l||c|c|c|c|}
\hline & tor,$+ e+$ jets & tor,$- e+$ jets & tor,$+ \mu+$ jets & tor,$- \mu+$ jets \\
\hline$N_{\Delta y>0}$ & 463 & 190 & 243 & 242 \\
$N_{\Delta y<0}$ & 353 & 151 & 181 & 186 \\
\hline Data $A_{\mathrm{FB}}^{l}(\%)$ & $13 \pm 3$ & $11 \pm 5$ & $14 \pm 5$ & $13 \pm 5$ \\
$\mathrm{~L}-\mathrm{T} A_{\mathrm{FB}}^{l}(\%)$ & $5 \pm 5$ & $4 \pm 12$ & $2 \pm 6$ & $5 \pm 6$ \\
\hline$A_{\mathrm{FB}}^{l}(\%)$ & $14 \pm 5$ & $11 \pm 7$ & $17 \pm 7$ & $14 \pm 7$ \\
\hline
\end{tabular}

Table 7.15 $A_{\mathrm{FB}}^{l}$ by toroid polarity and channel. 
effects are more likely to influence $A_{\mathrm{FB}}^{l}$. For the 774 events with positively charged leptons, $A_{\mathrm{FB}}^{l}=(13 \pm 5($ stat $)) \%$. For the 807 events with negatively charged leptons, $A_{\mathrm{FB}}^{l}=(16 \pm 5($ stat $)) \%$. The measurements with $A_{\mathrm{FB}}$ for positively and negatively charged leptons are $A_{\mathrm{FB}}=(8 \pm 5($ stat $)) \%$ and $A_{\mathrm{FB}}=(10 \pm 5($ stat $)) \%$, respectively. Neither the $\Delta y$-based asymmetry nor the lepton-based asymmetry is significantly dependent on the charge of the lepton. We conclude that the data shows no signs of mis-measurement by the detector or $\mathrm{CP}$ violation from new physics.

\subsection{Systematic Uncertainties}

There are multiple sources of systematic uncertainty that are taken into account. The systematic uncertainties are divided into three different categories: uncertainties on the predicted reconstruction-level asymmetries, uncertainties on the reconstruction-level measurements and uncertainties on unfolded asymmetries.

To evaluate the systematic uncertainties, each part of the analysis is performed with the relevant modification. These evaluations includes modifications to the $\Delta y$ and $q_{l} y_{l}$ distributions for the predicted asymmetries, modifications to the templates used for the background subtraction, modifications to the migration matrix and modifications to the selection efficiency.

The different types of variations used to calculate the systematic uncertainties are shown below, grouped into condensed categories for ease of display:

I Jet reco Uncertainties involved with the reconstruction of jets.

Jet efficiency Jet reconstruction and identification efficiency uncertainties are determined by varying the JetID data-MC scale factor, which removes extra jets from simulation, down by one standard deviation. This uncertainty is made symmetric.

Vertex confirmation In RunIIb, jets have an additional requirement of being vertex confirmed. An uncertainty is associated with the scale factor used to correct the rate of vertex confirmed jets in simulation to the one in data. This uncertainty is applied here and made symmetric. 
II JER/JES Uncertainties from jet energy scale and jet energy resolution.

Jet energy scale To determine the uncertainty on the jet energy scale, the JES correction is varied up and down, with the sum of the statistical and systematic uncertainties in data and $\mathrm{MC}$ added in quadrature.

Jet energy resolution To determine the uncertainty on the jet energy resolution, the JER correction is varied up and down by one standard deviation.

III Signal modeling Uncertainties associated with the simulation of signal events are included here.

Acceptance modeling Forward and backward events have different amounts of gluon radiation. This difference affects the relative selection efficiency between forward and backward events. One observable where gluon radiation plays a prominent role is the transverse momentum of the $t \bar{t}$ system. To understand the maximum effect of gluon radiation on the measurement, the measurement is carried out with this effect turned off.

Alternative signal model The systematics on the signal modeling are estimated by substituting MC@NLO+HERWIG with ALPGEN+PYTHIA for the signal simulation.

Additional collisions The luminosity profile in MC simulation is weighted to match the luminosity profile in data. To estimate the effect of additional collisions, this weight is turned off by setting it 1 .

Different top masses Two different signal files with top masses of $170 \mathrm{GeV}$ and $180 \mathrm{GeV}$ are used, instead of $172.5 \mathrm{GeV}$. These files are made with ALPGEN+PYTHIA and compared against the result for ALPGEN signal with a top mass of $172.5 \mathrm{GeV}$. To compute the difference, the variation is divided by the number of standard deviations from $172.5 \mathrm{GeV}$, according to the current uncertainty on the top mass, $1.4 \mathrm{GeV}$. The change for 170 $\mathrm{GeV}$ is divided by 2 and the change for $180 \mathrm{GeV}$ is divided by 4 .

The migration matrix requires $\mathrm{MC}$ samples with many simulated events. Instead of repeating the analysis per systematic variation as we do for the other uncertainties on unfolded events, this systematic uncertainty 
is only evaluated at the reconstruction level. At the production level, this systematic effect is estimated to have the same relative uncertainty.

Monte Carlo statistics The uncertainty due to limited MC statistics for the predicted asymmetry is taken into account.

IV $b$-Tagging Uncertainties associated with $b$-tagging.

Scale factors for heavy and light flavors The uncertainties on the $b, c$ and light scale factors used to match $b$-tagging rates in simulation to data are calculated by varying the corresponding SF by one standard deviation up and down. As the $b$ and $c$ flavored jets get the same SF assigned, we correlate the uncertainties on $b$ and $c$ jets by $100 \%$.

V Charge ID Uncertainties associated with the modeling of the charge misidentification rates.

Lepton charge misidentification The lepton charge misidentification rate is varied according to the uncertainties. This uncertainty is applied as a multiplicative factor that calibrates the fitted and unfolded asymmetries. The rate of misidentification of $0.00143_{-0.00143}^{+0.005}$, with an uncertainty of $0.5 \%$, yields a multiplicative factor of $1_{-0.0029}^{0.0101}$.

VI Background (Bg) subtraction Uncertainties associated with subtracting the background templates from the data.

$W+$ jets heavy flavor fraction A heavy flavor scale factor of 1.47 with a relative uncertainty of $15 \%$ is applied to $W c c$ and $W b b$ events.

$W+$ jets asymmetry The uncertainty on the asymmetry from the $W+$ jets due to limited MC statistics is taken into account.

Fake and true lepton selection rates The uncertainties on the fake lepton selection rate and true lepton selection rate used to evaluate multijet background are propagated to the multijet background yield, essentially varying $C$ and $1-C$ within the template fit.

Background subtraction Uncertainty from the normalization of the background templates is included with the statistical uncertainty for the 
fitted results. For the unfolded results, the multijet background template and the $W+$ jets background template are varied separately within their fitted uncertainties, which are almost completely uncorrelated.

VII Unfolding bias Uncertainty due to the bias from unfolding.

Calibration The results from regularized unfolding are calibrated with a bias factor. Uncertainties on this bias factor are propagated to the unfolded result to find the systematic uncertainty.

Not included in this list are the biases from the fitting procedure. The results of ensemble testing, described in Section 7.4.1, have additional systematic uncertainties of $+0.2 \%$ for $A_{\mathrm{FB}}$ and $+0.9 \%$ for $A_{\mathrm{FB}}^{l}$. The bias from the fitting procedure is in exact agreement with bias found in Reference [1]. The addition of the fitting bias for $A_{\mathrm{FB}}^{l}$ is not included in Reference [2], as it was not prepared in time for publication, and will be not be included here.

Tables D.3 and D.4 show the condensed systematic uncertainties for all measurements and predictions. Appendix D contains the systematic uncertainties broken down in more detail. As expected, the systematic uncertainties are much smaller than the statistical uncertainties for all of the measurements.

\begin{tabular}{|l|c|c|c|}
\hline & \multicolumn{3}{|c|}{ Absolute uncertainty (\%) } \\
Source & Reco. level & Gen. level \\
\hline Jet reco & \pm 0.3 & \pm 0.5 & \pm 1.0 \\
JES/JER & +0.5 & -0.5 & -1.3 \\
Signal modeling & \pm 0.3 & \pm 0.5 & $+0.3 /-1.6$ \\
b-tagging & - & \pm 0.1 & \pm 0.1 \\
Charge ID & - & +0.1 & $+0.2 /-0.1$ \\
Bg subtraction & - & \pm 0.1 & $+0.8 /-0.7$ \\
Unfolding Bias & - & - & $+1.1 /-1.0$ \\
\hline Total & $+0.7 /-0.5$ & $+0.8 /-0.9$ & $+1.8 /-2.6$ \\
\hline
\end{tabular}

Table 7.16 Systematic uncertainties on the $A_{\mathrm{FB}}$. Only uncertainties above $0.1 \%$ are listed. 


\begin{tabular}{|l|c|c|c|}
\hline & \multicolumn{3}{|c|}{ Absolute uncertainty (\%) } \\
& Reco. level & Gen. level \\
Source & Prediction & Measurement & Measurement \\
\hline \hline Jet reco & \pm 0.3 & \pm 0.1 & \pm 0.8 \\
JES/JER & +0.1 & -0.4 & $+0.1 /-0.6$ \\
Signal modeling & \pm 0.3 & \pm 0.5 & $+0.2 /-0.6$ \\
$b$-tagging & - & \pm 0.1 & \pm 0.1 \\
Charge ID & - & +0.1 & $+0.2 /-0.0$ \\
Bg subtraction & - & \pm 0.3 & \pm 0.6 \\
\hline Total & \pm 0.5 & \pm 0.7 & $+1.0 /-1.3$ \\
\hline
\end{tabular}

Table 7.17 Systematic uncertainties on the $A_{\mathrm{FB}}^{l}$. Only uncertainties above $0.1 \%$ are listed. 


\section{Conclusion}

We measure the forward-backward production asymmetry in top quark pair production using multiple techniques. We compare the asymmetries based on two different observable quantities to predictions from the MC@NLO next-to-leadingorder QCD event generator. One of the observable quantities, the rapidity difference between top and antitop quarks, $\Delta y$, takes into account the information from the entire $t \bar{t}$ decay. The other quantity, the charged lepton rapidity, $q_{l} y_{l}$, focuses on the part of the decay that is best measured by the detector. We correct both of the measurements for the effects of acceptance. We also correct $A_{\mathrm{FB}}$ for the effects of detector reconstruction.

At the reconstruction level, where asymmetries are formed from distributions of data in $\Delta y$ and $q_{l} y_{l}$ after background processes are subtracted, we measure $A_{\mathrm{FB}}=(9.2 \pm 3.7) \%$ and $A_{\mathrm{FB}}^{l}=(14.2 \pm 3.8) \%$. At the production level, where the distributions of background-subtracted data are corrected for the effects acceptance and detector resolution, we measure $A_{\mathrm{FB}}=(19.6 \pm 6.5) \%$ and $A_{\mathrm{FB}}^{l}=(15.2 \pm 4.0) \%$. These asymmetries are larger than those predicted by MC@NLO, but there is not yet enough statistical strength to decisively claim a divergence of the data from theoretical predictions.

In addition to measuring the inclusive $t \bar{t}$ asymmetry from all events that pass the selection criteria, we perform a differential measurement $A_{\mathrm{FB}}$ with respect to the invariant mass of the $t \bar{t}$ system. We did not find a statistically significant dependence of $A_{\mathrm{FB}}$ on $m_{t \bar{t}}$ compared to the predictions from MC@NLO, but our measurements also do not disagree with the latest results from the CDF collaboration [33]. 


\subsection{Projections for the Future}

Now that the results have been presented and explained, predictions can be made for future measurements, specifically at the Tevatron. A simple way to predict what kind of significance the measurement could have in the future is to double the statistics of the events in the ensemble testing, which is more or less what will happen for the analysis of the full Tevatron dataset. Figure 8.1 shows the uncertainty bands for $\sim 10 \mathrm{fb}^{-1}$ of data, along with the central values for the current measurement. Note that this plot is somewhat misleading, due the use of the MC@NLO generator, which is known to have asymmetries that are slightly lower than other predictions. As the reader can see, if the new measured values remain where the current data are, the future could become even more interesting.

We have many future plans for continuing this analysis beyond a mere update using the data from the entirety of Run II of the Tevatron collected by D $\varnothing$. These plans are still in the development stage and focus on three different aims: increasing the number of $t \bar{t}$ events, investigating the behavior of the asymmetry in a variety of situations and conducting an even more thorough set of studies of the asymmetries predicted by background processes. We encourage interested readers to stay tuned.
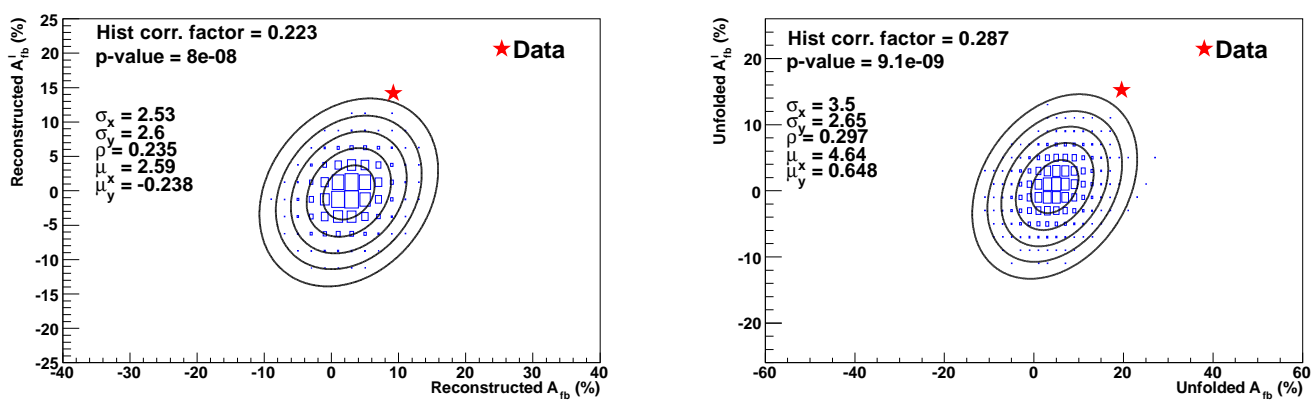

Figure 8.1 Correlation plots with sigma contour lines for different asymmetries. The plots on the left compare reconstruction-level asymmetries and the plots on the right compare unfolded asymmetries. These plots are a projection for the future with the amount of data doubling and the measured asymmetries staying the same. The red lines represent the current values measured from data. 


\section{Bibliography}

[1] Abazov, V. et al., "First measurement of the forward-backward charge asymmetry in top quark pair production," Phys.Rev.Lett., Vol. 100, 2008, pp. 142002 .

[2] Abazov, V. M. et al., "Forward-backward asymmetry in top quark-antiquark production," 2011.

[3] Geiger, H. and Marsden, E., "On a Diffuse Reflection of the $\alpha$-Particles," Proc. Roy. Soc., Vol. 82, 1909, pp. 495-500.

[4] Lee, T. D. and Yang, C. N., "Question of Parity Conservation in Weak Interactions," Phys. Rev., Vol. 104, Oct 1956, pp. 254-258.

[5] Wu, C. S., Ambler, E., Hayward, R. W., Hoppes, D. D., and Hudson, R. P., "Experimental Test of Parity Conservation in Beta Decay," Phys. Rev., Vol. 105, Feb 1957, pp. 1413-1415.

[6] Garwin, R. L., Lederman, L. M., and Weinrich, M., "Observations of the Failure of Conservation of Parity and Charge Conjugation in Meson Decays: the Magnetic Moment of the Free Muon," Phys. Rev., Vol. 105, Feb 1957, pp. 1415-1417.

[7] Gross, D. J. and Wilczek, F., "Ultraviolet Behavior of Non-Abelian Gauge Theories," Phys. Rev. Lett., Vol. 30, Jun 1973, pp. 1343-1346.

[8] Gross, D. J., "Twenty five years of asymptotic freedom," Nucl.Phys.Proc.Suppl., Vol. 74, 1999, pp. 426-446.

[9] Halzen, F. and Martin, A. D., "Quarks and Leptons," Wiley, 1984. 
[10] Gockeler, M., Horsley, R., Irving, A., Pleiter, D., Rakow, P., et al., "A Determination of the Lambda parameter from full lattice QCD," Phys.Rev., Vol. D73, 2006, pp. 014513.

[11] Davies, C. et al., "High precision lattice QCD confronts experiment," Phys.Rev.Lett., Vol. 92, 2004, pp. 022001.

[12] de Troconiz, J. and Yndurain, F., "Calculation of alpha-bar (QED) on the Z," Phys.Rev., Vol. D65, 2002, pp. 093002.

[13] Lepage, G., "Lattice QCD for novices," 1998, pp. 49-90.

[14] Abe, F. et al., "Observation of Top Quark Production in $\bar{p} p$ Collisions with the Collider Detector at Fermilab," Phys. Rev. Lett., Vol. 74, Apr 1995, pp. 2626-2631.

[15] Abachi, S. et al., "Observation of the Top Quark," Phys. Rev. Lett., Vol. 74, Apr 1995, pp. 2632-2637.

[16] Nakamura, K. et al., "The Review of Particle Physics," J. Phys. G 37, 075021, 2010.

[17] Ahrens, V., Ferroglia, A., Neubert, M., Pecjak, B. D., and Yang, L. L., "Renormalization-Group Improved Predictions for Top-Quark Pair Production at Hadron Colliders," JHEP, Vol. 1009, 2010, pp. 097.

[18] Ahrens, V., Ferroglia, A., Neubert, M., Pecjak, B., and Yang, L., "Top-Quark Pair Production Beyond Next-to-Leading Order," Nucl.Phys.Proc.Suppl., Vol. 205-206, 2010, pp. 48-53.

[19] Moch, S. and Uwer, P., "Theoretical status and prospects for top-quark pair production at hadron colliders," Phys.Rev., Vol. D78, 2008, pp. 034003.

[20] Langenfeld, U., Moch, S., and Uwer, P., "Measuring the running top-quark mass," Phys.Rev., Vol. D80, 2009, pp. 054009.

[21] Aliev, M., Lacker, H., Langenfeld, U., Moch, S., Uwer, P., et al., "HATHOR: HAdronic Top and Heavy quarks crOss section calculatoR," Comput.Phys.Commun., Vol. 182, 2011, pp. 1034-1046. 
[22] Kidonakis, N. and Vogt, R., "Next-to-next-to-leading order soft gluon corrections in top quark hadroproduction," Phys.Rev., Vol. D68, 2003, pp. 114014.

[23] Kidonakis, N., "Next-to-next-to-leading soft-gluon corrections for the top quark cross section and transverse momentum distribution," Phys.Rev., Vol. D82, 2010, pp. 114030.

[24] Abazov, V. M. et al., "Measurement of the top quark pair production cross section in the lepton + jets channel in proton-antiproton collisions at $\sqrt{s}=$ $1.96 \mathrm{TeV}, "$ Phys. Rev. D, Vol. 84, Jul 2011, pp. 012008.

[25] Kidonakis, N., "Single top quark production at the Fermilab Tevatron: Threshold resummation and finite-order soft gluon corrections," Phys. Rev. D, Vol. 74, Dec 2006, pp. 114012.

[26] Abazov, V. M. et al., "Measurements of single top quark production cross sections and $\left|V_{t b}\right|$ in $p \bar{p}$ collisions at $\sqrt{s}=1.96 \mathrm{TeV}$," 2011, Long author list - awaiting processing.

[27] Abazov, V. M. et al., "Precision Measurement of the Ratio $\mathrm{B}(t \rightarrow$ $W b) / \mathrm{B}(t \rightarrow W q)$ and Extraction of $V_{\mathrm{tb}}$," Phys. Rev. Lett., Vol. 107, Sep 2011, pp. 121802.

[28] Kühn, J. H. and Rodrigo, G., "Charge Asymmetry in Hadroproduction of Heavy Quarks," Phys. Rev. Lett., Vol. 81, Jul 1998, pp. 49-52.

[29] Kühn, J. H. and Rodrigo, G., "Charge asymmetry of heavy quarks at hadron colliders," Phys. Rev. D, Vol. 59, Feb 1999, pp. 054017.

[30] Nason, P., Dawson, S., and Ellis, R., "The total cross section for the production of heavy quarks in hadronic collisions," Nuclear Physics B, Vol. 303, No. 4, 1988, pp. $607-633$.

[31] Halzen, F., Hoyer, P., and Kim, C., "Forward-backward asymmetry of hadroproduced heavy quarks in QCD," Physics Letters B, Vol. 195, No. 1, 1987, pp. $74-77$. 
[32] Aaltonen, T. et al., "Forward-Backward Asymmetry in Top Quark Production in $p \bar{p}$ Collisions at sqrts $=1.96 \mathrm{TeV}, "$ Phys.Rev.Lett., Vol. 101, 2008, pp. 202001.

[33] Aaltonen, T. et al., "Evidence for a Mass Dependent Forward-Backward Asymmetry in Top Quark Pair Production," Phys.Rev., Vol. D83, 2011, pp. 112003.

[34] Aaltonen, T. et al., "Measurement of the Forward Backward Asymmetry in Top Pair Production in the Dilepton Decay Channel using $5.1 \mathrm{fb}^{-1}$ " May be found at http://www-cdf.fnal.gov/physics/new/top/2011/DilAfb/ cdfpubnote.pdf.

[35] Bowen, M., Ellis, S., and Rainwater, D., "Standard model top quark asymmetry at the Fermilab Tevatron," Phys.Rev., Vol. D73, 2006, pp. 014008.

[36] Dittmaier, S., Uwer, P., and Weinzierl, S., "NLO QCD corrections to t anti-t + jet production at hadron colliders," Phys.Rev.Lett., Vol. 98, 2007, pp. 262002.

[37] Melnikov, K. and Schulze, M., "NLO QCD corrections to top quark pair production in association with one hard jet at hadron colliders," Nucl.Phys., Vol. B840, 2010, pp. 129-159.

[38] Ahrens, V., Ferroglia, A., Neubert, M., Pecjak, B. D., and Yang, L. L., "The top-pair forward-backward asymmetry beyond NLO," Phys.Rev., Vol. D84, 2011, pp. 074004.

[39] Hollik, W. and Pagani, D., "The electroweak contribution to the top quark forward-backward asymmetry at the Tevatron," 2011, * Temporary entry *.

[40] Pecjak, B., "Top-quark charge asymmetry at hadron colliders within the SM," TOP 2011 Conference Talk, 2011, https: //indico.cern. ch/getFile.py/access? contribId=41\&sessionId= 34\&res Id=0\&material Id=slides\&conf $I d=144537$.

[41] Frixione, S. and Webber, B. R., "Matching NLO QCD computations and parton shower simulations," JHEP, Vol. 06, 2002, pp. 029. 
[42] Frixione, S., Nason, P., and Webber, B. R., "Matching NLO QCD and parton showers in heavy flavour production," JHEP, Vol. 08, 2003, pp. 007.

[43] Campbell, J. M. and Ellis, R. K., "Update on vector boson pair production at hadron colliders," Phys. Rev. D, Vol. 60, Nov 1999, pp. 113006.

[44] Bernreuther, W. and Si, Z.-G., "Distributions and correlations for top quark pair production and decay at the Tevatron and LHC." Nucl.Phys., Vol. B837, 2010, pp. 90-121.

[45] Aguilar Saavedra, J. A., "Overview of models for the $t \bar{t}$ asymmetry," TOP 2011 Conference Talk, 2011, https://indico.cern.ch/getFile. py/access? contribId=44\&session Id=36\&res $I d=0 \&$ material $I d=$ slides\&conf Id=144537.

[46] Krohn, D., Liu, T., Shelton, J., and Wang, L.-T., "A Polarized View of the Top Asymmetry," Phys.Rev., Vol. D84, 2011, pp. 074034.

[47] Abazov, V. et al., "The upgraded D $\varnothing$ detector," Nuclear Instruments and Methods in Physics Research Section A: Accelerators, Spectrometers, Detectors and Associated Equipment, Vol. 565, No. 2, 2006, pp. 463 - 537.

[48] "Home page for Tevatron Run II handbook," http://www-bd.fnal.gov/ lug/runII_handbook/RunII_index.html.

[49] Abe, F. et al., "The CDF detector: an overview," Nucl. Instr. Meth., Vol. A271, 1988, pp. 387-403.

[50] "Accelerator Concepts Rookie Book," http://www-bdnew.fnal.gov/ operations/rookie_books/rbooks.html.

[51] "Fermilab Tevatron website," http://www.fnal.gov/pub/science/accelerator/.

[52] Abachi, S. et al., "The D $\varnothing$ detector," Nuclear Instruments and Methods in Physics Research Section A: Accelerators, Spectrometers, Detectors and Associated Equipment, Vol. 338, No. 23, 1994, pp. 185 - 253.

[53] Abachi, S. et al., "The D0 upgrade: The Detector and its physics," 1996, FERMILAB-PUB-96-357-E. 
[54] "D $\varnothing$ fact sheet," http://www-d0.fnal.gov/public/popular-lectures/ D0_Fact_Sheet.ppt.

[55] "The DØ Silicon Tracker Technical Design Report," 1994, http://www-d0. fnal.gov/ lipton/tdr_final.ps.

[56] Angstadt, R. et al., "The layer 0 inner silicon detector of the D0 experiment," Nuclear Instruments and Methods in Physics Research A, Vol. 622, Oct. 2010, pp. 298-310.

[57] "DØ Central Fiber Tracker Description Page," 2005, http://d0server1. fnal.gov/projects/scifi/cft_description.html.

[58] Brzezniak, J., Fast, R., Krempetz, K. J., Kristalinski, A., Lee, A., et al., "Conceptual design of a 2-Tesla superconducting solenoid for the Fermilab D0 detector upgrade," 1994.

[59] Bernardi, G. et al., "Improvements from the T42 Algorithm on Calorimeter Objects Reconstruction," 2004, Internal DØ Note 4335.

[60] Edwards, T. L. et al., "Determination of the effective inelastic $p \bar{p}$ crosssection for the D0 Run II luminosity measurement," 2004.

[61] Jenni, P., Nessi, M., Nordberg, M., and Smith, K., ATLAS high-level trigger, data-acquisition and controls: Technical Design Report, Technical Design Report ATLAS, CERN, Geneva, 2003.

[62] Abazov, V. et al., "D0 Run IIB upgrade technical design report," 2002.

[63] "DØ Run II Bests webpage," 2012, http://www-d0.fnal.gov/runcoor/ run2best.html.

[64] Borissov, G., "Ordering a Chaos or ... Technical Details of AA tracking," All DØ Meeting February 28, 2003, http://www-d0.fnal.gov/atwork/ $\mathrm{adm} / \mathrm{d} 0$ _private/2003-02-28/adm_talk.ps.

[65] Khanov, A., "HTF: histogramming method for finding tracks. The algorithm description." 2000, Internal DØ Note 3778. 
[66] R. and Fr?hwirth, "Application of Kalman filtering to track and vertex fitting," Nuclear Instruments and Methods in Physics Research Section A: Accelerators, Spectrometers, Detectors and Associated Equipment, Vol. 262, No. 23, 1987, pp. $444-450$.

[67] Greenlee, H., "The DØ Interacting Propagator," 2003, Internal DØ Note 4293.

[68] Hough, P. V. C., "Machine Analysis of Bubble Chamber Pictures," Proc. Int. Conf. High Energy Accelerators and Instrumentation, 1959.

[69] Schwartzman, A. and Tully, C., "Primary Vertex Reconstruction by Means of Adaptive Vertex Fitting," 2005, Internal DØ Note 4918.

[70] Schwartzman, A. and Narain, M., "Probabilistic Primary Vertex Selection," 2002, Internal DØ Note 4042.

[71] Kumar, A. et al., "Electron Likelihood Study," 2005, Internal DØ Note 4769.

[72] "Run II DØ Muon Identification," 2008, http://www-d0.fnal.gov/phys_ id/muon_id/d0_private/muonid_dh.html.

[73] Brandt, O. et al., "Muon Identification Certification for the Summer 2009 Extended Dataset (Run IIb-1 and -2)," 2010, Internal DØ Note 6025.

[74] Blazey, G. C., Dittmann, J. R., Ellis, S. D., Elvira, V., Frame, K., et al., "Run II jet physics," 2000, pp. 47-77.

[75] Emmanuel Busato, B. A., "Jet Algorithms in the D $\varnothing$ Run II Software: Description and User's Guide," 2004, Internal DØ Note 4457.

[76] Harel, A., "Jet ID Optimization," 2005, Internal DØ Note 4919.

[77] "DØ Final RunIIa Jet Energy Scale," 2008, http://www-d0.fnal.gov/ phys_id/jes/public_RunIIa/.

[78] R. Demina, A. Khanov, F. R., "b-tagging with Counting Signed Impact Parameter method," 2003, Internal DØ Note 4049. 
[79] Daniel Bloch, Benoit Clement, D. G. et al., "Performance of the JLIP btagger in p14," 2004, Internal DØ Note 4348.

[80] Schwartzman, A. and Narain, M., "b-quark jet identification via Secondary Vertex Reconstruction," 2003, Internal DØ Note 4080.

[81] Schwartzman, A. and Narain, M., "Secondary Vertex b-tagging using the Kalman Filter Algorithm," 2001, Internal DØ Note 3909.

[82] Schwartzman, A. and Narain, M., "Secondary Vertex Reconstruction using the Kalman Filter Algorithm," 2001, Internal DØ Note 3908.

[83] Hanagaki, K. and Kasper, J., "Identification of b-jet by Soft Muon," 2005, Internal DØ Note 4867.

[84] Scanlon, T., "A Neural Net b-tagging Tool," 2005, Internal DØ Note 4889.

[85] "DØ Top Triggers," 2008, http://www-d0.fnal.gov/Run2Physics/top/ d0_private/wg/triggers/triggers.html.

[86] Mangano, M. L., Moretti, M., Piccinini, F., Pittau, R., and Polosa, A. D., "ALPGEN, a generator for hard multiparton processes in hadronic collisions," JHEP, Vol. 0307, 2003, pp. 001.

[87] Sjostrand, T., Mrenna, S., and Skands, P. Z., "PYTHIA 6.4 Physics and Manual," JHEP, Vol. 05, 2006, pp. 026.

[88] Corcella, G., Knowles, I., Marchesini, G., Moretti, S., Odagiri, K., et al., "HERWIG 6.5 release note," 2002.

[89] Pumplin, J. et al., "New generation of parton distributions with uncertainties from global QCD analysis," JHEP, Vol. 07, 2002, pp. 012.

[90] Aaron, F. D. et al., "Combined Measurement and QCD Analysis of the Inclusive ep Scattering Cross Sections at HERA," JHEP, Vol. 01, 2010, pp. 109.

[91] Fisyak, Y. and Womersley, J., "D0gstar: DØ GEANT Simulation of the Total Apparatus Response," 1997, Internal DØ Note 3191. 
[92] Agostinelli, S. et al., "Geant4a simulation toolkit," Nuclear Instruments and Methods in Physics Research Section A: Accelerators, Spectrometers, Detectors and Associated Equipment, Vol. 506, No. 3, 2003, pp. 250 - 303.

[93] Hoeche, S., Krauss, F., Lavesson, N., Lonnblad, L., Mangano, M., et al., "Matching parton showers and matrix elements," 2006.

[94] Boos, E. et al., "CompHEP 4.4: Automatic computations from Lagrangians to events," Nucl.Instrum.Meth., Vol. A534, 2004, pp. 250-259.

[95] Schellman, H., "The longitudinal shape of the luminous region at DØ," 2006, Internal D $\varnothing$ Note 5142.

[96] Andersson, B., Gustafson, G., Ingelman, G., and Sjostrand, T., "Parton Fragmentation and String Dynamics," Phys. Rept., Vol. 97, 1983, pp. 31145.

[97] Bowler, M. G., " $e^{+} e^{-}$Production of Heavy Quarks in the String Model," 1981, Z. Phys. C11 169.

[98] Abe, K. et al., "Measurement of the b quark fragmentation function in Z0 decays," Phys.Rev., Vol. D65, 2002, pp. 092006.

[99] "DØ Event Displays and Illustrations," 2012, http://www-d0.fnal.gov/ Run2Physics/displays/.

[100] Snyder, S., Measurement of the Top Quark Mass at DØ, Ph.D. thesis, Stony Brook University, Stony Brook, NY, 1995, http://www-d0.fnal. gov/results/publications_talks/thesis/snyder/thesis-html.html.

[101] Abazov, V. et al., "Measurement of the $t \bar{t}$ production cross section in $p \bar{p}$ collisions at $\sqrt{s}=1.96-\mathrm{TeV}$ using kinematic characteristics of lepton + jets events," Phys.Rev., Vol. D76, 2007, pp. 092007.

[102] Cowan, G., Statistical Data Analysis, Oxford University Press, 1998.

[103] Bohm, G. and Zech, G., Introduction to statistics and data analysis for physicists, DESY, 2010. 
[104] James, F., "TMinuit Class in ROOT," May be found at http://root.cern.ch/root/html/TMinuit.html.

[105] Abazov, V. M. et al., "Measurement of the $\mathrm{W}$ boson helicity in top quark decays using $5.4 \mathrm{fb}^{-1}$ of $\boldsymbol{p} \overline{\boldsymbol{p}}$ collision data," Phys. Rev., Vol. D83, 2011, pp. 032009.

[106] Abazov, V. M. et al., "Precise study of the $Z / \gamma^{*}$ boson transverse momentum distribution in $p \bar{p}$ collisions using a novel technique," Phys.Rev.Lett., Vol. 106, 2011, pp. 122001.

[107] "TUnfold home page," http://root.cern.ch/root/html/TUnfold.html.

[108] Schmidt, S., "TUnfold overview," http://www.desy.de/ sschmitt/tunfold.htmll.

[109] Hansen, P. C., "The L-Curve and its Use in the Numerical Treatment of Inverse Problems," in Computational Inverse Problems in Electrocardiology, ed. P. Johnston, Advances in Computational Bioengineering, WIT Press, 2000, pp. 119-142.

[110] Cowan, G., "Statistics," http://pdg.lbl.gov/2011/reviews/rpp2011-revstatistics.pdf.

[111] Johnson, R. A. and Wichern, D. W., Applied Multivariate Statistical Analysis, Prentice Hall, 2001.

[112] Abazov, V. et al., "Measurement of the muon charge asymmetry from $W$ boson decays," Phys.Rev., Vol. D77, 2008, pp. 011106. 


\section{A Derivation of statistical uncertainty for weighted events}

Recall that the total number of events for weighted events is:

$$
N_{t o t}\left(n_{i}, w_{i}\right)=\Sigma_{i=0}^{N} n_{i} w_{i}
$$

Usually each $n_{i}$ is equal to one, but the calculation is aided by the inclusion of this factor. To find the uncertainty, partial derivatives must be taken for each $n_{i}$ and $w_{i}$, and added in quadrature.

$$
\left(\Delta N_{t o t}\right)^{2}=\Sigma_{i=0}^{N}\left(\frac{\partial N}{\partial n_{i}} \delta n_{i}\right)^{2}+\Sigma_{i=0}^{N}\left(\frac{\partial N}{\partial w_{i}} \delta w_{i}\right)^{2}
$$

The last part of the equation $\left(\frac{\partial N}{\partial w_{i}}\right)$ is essentially considered in the systematic uncertainties (variations in b-tagging weights, etc.), so only the first part of the

equation $\left(\frac{\partial N}{\partial n_{i}}\right)$ is considered for the statistical uncertainty. The uncertainty on the number of events is the usual Poisson uncertainty of $\sqrt{n_{i}}$, which leads to:

$$
\left(\Delta N_{t o t}\right)^{2}=\Sigma_{i=0}^{N}\left(w_{i} \sqrt{n_{i}}\right)^{2}
$$

Therefore, the square of the statistical uncertainty for weighted events is the sum of the squares of the weights:

$$
\left(\Delta N_{t o t}\right)^{2}=\Sigma_{i=0}^{N} n_{i} w_{i}^{2}=\Sigma_{i=0}^{N} w_{i}^{2}
$$




\section{B Dilution}

At the time of the first DØ publication on the asymmetry [1], MC@NLO was not fully integrated into the $\mathrm{D} \varnothing$ software framework. So events generated with MC@NLO could not be processed by the DØ detector simulation or reconstruction sequences. Instead a parameterization was made to quantify the effect of reconstruction on the asymmetry using $t \bar{t}$ events that were simulated with ALPGEN+PYTHIA, and processed by the simulation of the DØ detector and the reconstruction chain. Because the asymmetry is the difference between the fraction of forward events and the fraction of backward events, only the sign of the $\Delta y$, which separates forward from backward, matters.

When the sign of $\Delta y$ in an event is the same for both the true generated value of $\Delta y$ and reconstructed value of $\Delta y$ the event is properly reconstructed for the purpose of the asymmetry measurement. Events that reconstruct the sign of $\Delta y$ correctly have

$$
\left(q_{l}\left(y_{t, l e p}-y_{t, h a d}\right)\right)_{g e n} \times\left(q_{l}\left(y_{t, l e p}-y_{t, h a d}\right)\right)_{\text {reco }}=1,
$$

while events that improperly reconstruct the sign of $\Delta y$ have

$$
\left(q_{l}\left(y_{t, l e p}-y_{t, h a d}\right)\right)_{g e n} \times\left(q_{l}\left(y_{t, l e p}-y_{t, h a d}\right)\right)_{\text {reco }}=-1 \text {. }
$$

The reasons for improper reconstruction of the sign $\Delta y$ can be either the misidentification of the lepton charge, a small effect, or the improper reconstruction of the sign of $y_{t, l e p}-y_{t, h a d}$, a much larger effect. The incorrect assignment of jets to partons by the kinematic fit is the main factor for the latter reason. We define 

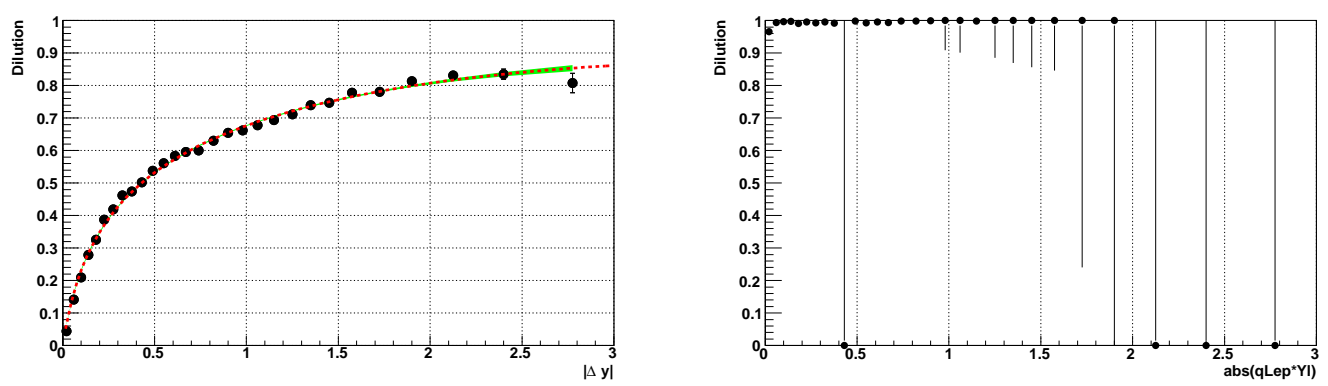

Figure B.1 Dilution for $A_{\mathrm{FB}}$ using reconstructed $t \bar{t}$ events from MC@NLO. The dilution curves are shown separately for the fully reconstructed $\Delta y$ (left), and for the signed lepton rapidty, $\left|q_{l} y_{l}\right|$ (right).

the probability, $P$, for the sign of $\Delta y$ to be constructed correctly at a given value of $\Delta y$. The dilution, $\mathcal{D}$, can be defined as:

$$
\mathcal{D}=2 P-1
$$

In Figure B.1, the dilution is shown for the different channels. For the observable used to construct the lepton-based $A_{\mathrm{FB}}^{l}$, the dilution is essentially 1 across all values of $\left|y_{l}\right|$ and effects from reconstruction have a small role, as shown in the right plot. At larger magnitudes of $\Delta y$ the dilution factor is large and events are reconstructed with the proper sign about $90 \%$ of the time. As the magnitude of $\Delta y$ gets smaller, especially when $|\Delta y|<0.5$, the sign of the $\Delta y$ is no longer well resolved and the dilution quickly reduces to zero.

The following function is fit to the dilution curve:

$$
D\left(\left|\Delta y_{\text {gen }}\right|\right)=c_{0} \ln \left(1+c_{1}\left|\Delta y_{\text {gen }}\right|+c_{2}\left|\Delta y_{\text {gen }}\right|^{2}\right)
$$

Table B.1 contains the parameters from these fits.

\begin{tabular}{lccc} 
Reco version & $c_{0}$ & $c_{1}$ & $c_{2}$ \\
\hline Run IIa & 0.2317 & 18.50 & 0.0664 \\
Run IIb & 0.2374 & 16.87 & -1.731 \\
Combined & 0.2372 & 17.09 & -1.555
\end{tabular}

Table B.1 Dilution Parameters. 


\section{B.0.1 Using the prediction as a cross check for dilution}

The predicted reconstruction level asymmetry, $A_{\mathrm{FB}}^{\text {pred }}$, can be determined without processing events through the full detector simulation by using the prescription presented in the first $\mathrm{D} \varnothing$ measurement [1]:

$$
A_{\mathrm{FB}}^{\text {pred }}=\int_{0}^{\infty} A_{\mathrm{FB}}(\Delta y) \mathcal{D}(\Delta y)[g(\Delta y)+g(-\Delta y)] d \Delta y
$$

where $\mathcal{D}$ is the function from Section B and $g(\Delta y)$ is the event density at a given value of $\Delta y$, after acceptance has been taken into account. The functions for $g(|\Delta y|)$ are essentially the same before and after selection with $0.94 e^{\left(-\frac{x^{2}}{1.5}\right)}$. Using the event density, $A_{\mathrm{FB}}(\Delta y)=\frac{g(\Delta y)-g(-\Delta y)}{g(\Delta y)+g(-\Delta y)}$. Two other functions are still needed to compute the prediction: $A_{\mathrm{FB}}(\Delta y)$ and $g(\Delta y)+g(-\Delta y)=g(|\Delta y|)$. The functions for $A_{\mathrm{FB}}(\Delta y)$ are shown in Figure B.2. Since the complete selection requirements are not present outside of the $\mathrm{D} \varnothing$ detector simulation, "particle" jets, or jets made with particles directly the $t \bar{t}$ decay from MC@NLO+HERWIG, are in lieu of actual jets.
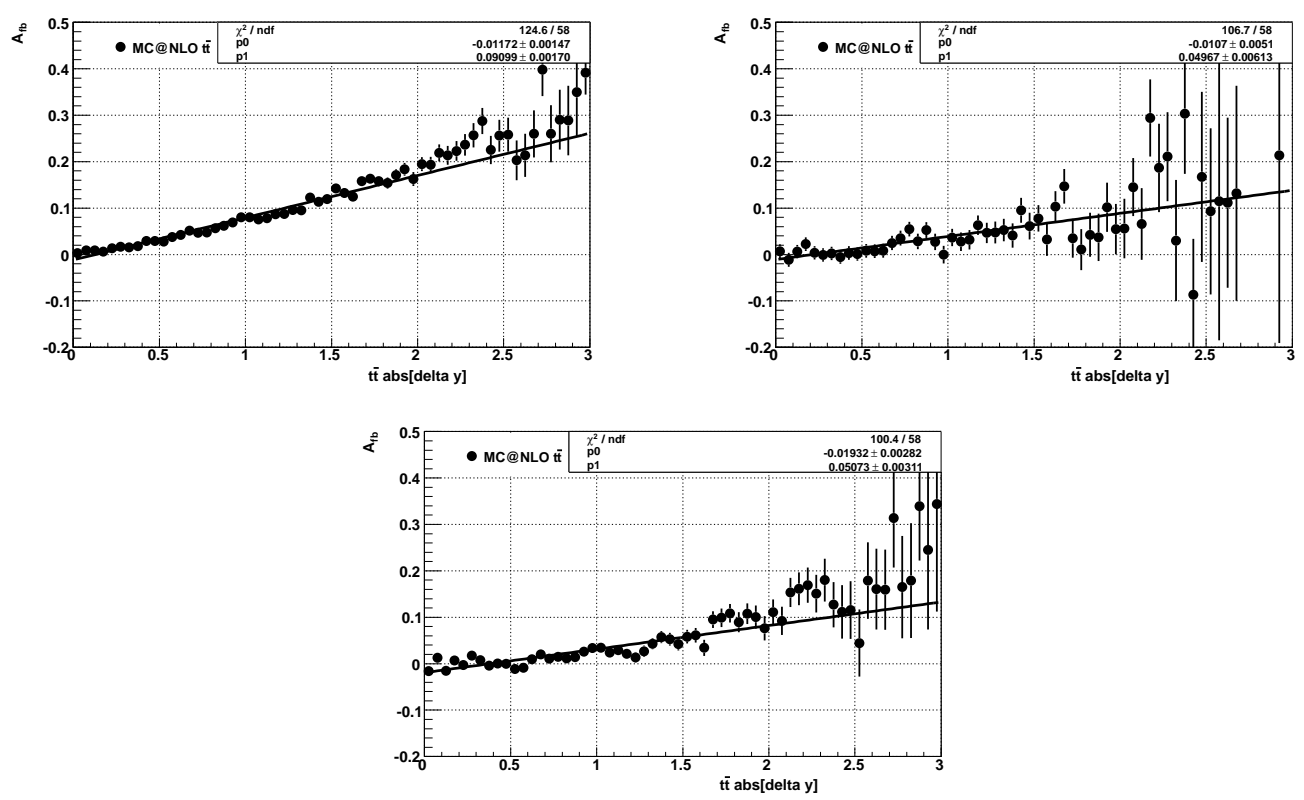

Figure B.2 Fitted functions for for $A_{\mathrm{FB}}(\Delta y)$ with different levels of selection. $A_{\mathrm{FB}}(\Delta y)$ before selection (top, left), $A_{\mathrm{FB}}(\Delta y)$ after the full selection (top, right) and $A_{\mathrm{FB}}(\Delta y)$ after parameterized selection with "particle" jets (bottom). 
Using the values from fits to $A_{\mathrm{FB}}(\Delta y), g(|\Delta y|)$ and $D\left(\left|\Delta y_{\text {gen }}\right|\right)$, we test the ability of the dilution prescription to correctly predict the asymmetry. The results of this prescription are shown in Table B.2. The dilution prescription predicted the asymmetry within the uncertainties given in Reference [1], although there was bias of $-1 \%$. For theories that are not incorporated into the full $\mathrm{D} \emptyset$ detector simulation, the dilution prescription offers a relatively good, fast way to get a reconstruction-level prediction. Note that this procedure has only been tested in this one case.

\begin{tabular}{cll} 
Selection & $A_{\mathrm{FB}}$ from Eqn. B.5 (\%) & Actual $A_{\mathrm{FB}}$ pred. (\%) \\
\hline None & 5.3 & 4.9 \\
Full selection (no dilution) & 3.6 & 2.4 \\
Full selection & 1.7 & 2.4 \\
Selection with "particle" jets & 1.3 & 2.4
\end{tabular}

Table B.2 Comparison between asymmetries predicted with Equation B.5 (center column) and the full detector simulation (right column). 


\section{Template fits}

This appendix contains the $t \bar{t}$ and $W+$ jets templates as well as the corresponding fits used by the discriminant. Because of the differences between Run IIa and Run IIb, different fits are used for each run period. Within a given run period, $e+$ jets and $\mu+$ jets events are included in the same fits. Figure C.1 shows the templates and fits for Run IIa. Figure C.2 shows the templates and fits for Run IIb. 

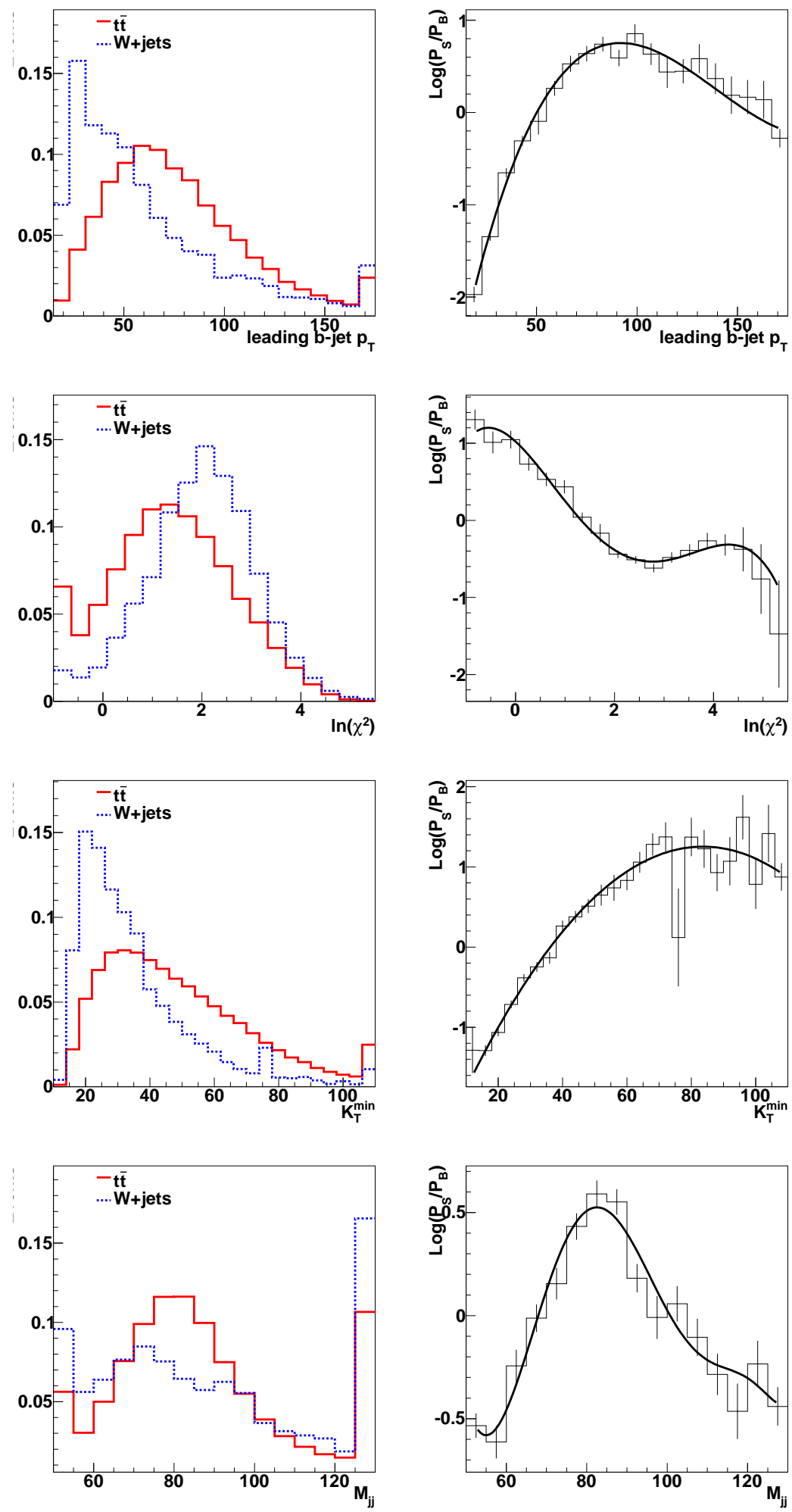

Figure C.1 Run IIa template fits. The left plots show the distributions of the input variables used in the likelihood discriminant in simulated $t \bar{t}$ events (red curve) and $W+$ jets events (dashed blue curve). The right plots show the logarithms of the ratios of these distributions (points and histogram), and the fit to them (curve). Overflows are shown in the extreme bins. 

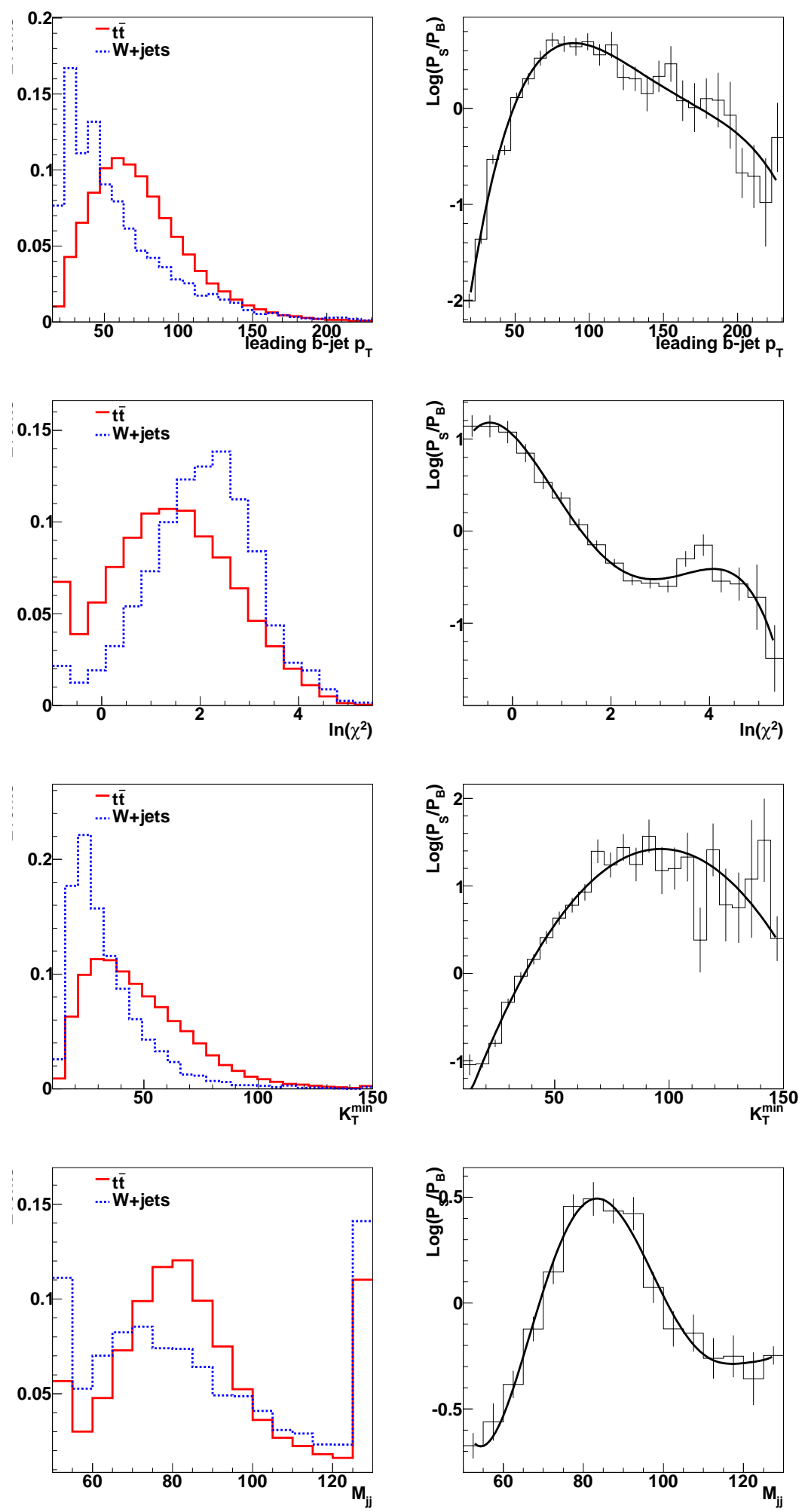

Figure C.2 Run IIb template fits. The left plots show the distributions of the input variables used in the likelihood discriminant in simulated $t \bar{t}$ events (red curve) and $W+$ jets events (dashed blue curve). The right plots show the logarithms of the ratios of these distributions (points and histogram), and the fit to them (curve). Overflows are shown in the extreme bins. 


\section{Complete systematics}

In the body of the thesis an abbreviated systematics table was shown. In this appendix the tables in their full glory are shown with each individual systematic effect and the contribution to the various asymmetries.

\section{D.0.2 Systematic uncertainties on predicted asymmetries}

\begin{tabular}{|c|c|c|c|c|}
\hline Source & $\Delta^{+}($in $\%)$ & $\Delta^{-}($in $\%)$ & $\sigma^{+}($in $\%)$ & $\sigma^{-}($in $\%)$ \\
\hline Jet Efficiency & \multicolumn{2}{|c|}{+0.2} & +0.2 & -0.2 \\
\hline Vertex Confirmation & \multicolumn{2}{|c|}{-0.1} & +0.1 & -0.1 \\
\hline Additional Collisions & \multicolumn{2}{|c|}{+0.2} & +0.2 & -0.2 \\
\hline Jet Enetry Resolution & +0.2 & +0.1 & +0.2 & -0.0 \\
\hline Jet Energy Scale & +0.1 & +0.4 & +0.4 & -0.0 \\
\hline Scale Factor for Heavy Flavor & -0.0 & +0.1 & +0.1 & -0.0 \\
\hline Scale Factor for Light Flavor & -0.0 & +0.0 & +0.0 & -0.0 \\
\hline Total & & & +0.6 & -0.4 \\
\hline
\end{tabular}

Table D.1 Absolute systematic uncertainties on $A_{\mathrm{FB}}^{\mathrm{pred}}$. The 2nd and 3rd columns list the effect of each systematic variation on $A_{\mathrm{FB}}^{\mathrm{pred}}$, and the 4 th and 5th columns list the resulting up and down uncertainties. 


\begin{tabular}{|c|c|c|c|c|}
\hline Source & $\overline{\Delta^{+}(\text {in } \%)}$ & $\Delta^{-}($in $\%)$ & $\sigma^{+}($in $\%)$ & $\overline{\sigma^{-}(\text {in } \%)}$ \\
\hline Jet Efficiency & \multicolumn{2}{|c|}{+0.1} & +0.1 & -0.1 \\
\hline Vertex Confirmation & \multicolumn{2}{|c|}{-0.3} & +0.3 & -0.3 \\
\hline Additional Collisions & \multicolumn{2}{|c|}{+0.1} & +0.1 & -0.1 \\
\hline Jet Enetry Resolution & +0.1 & -0.0 & +0.1 & -0.0 \\
\hline Jet Energy Scale & +0.0 & +0.1 & +0.1 & -0.0 \\
\hline Scale Factor for Heavy Flavor & -0.0 & +0.0 & +0.0 & -0.0 \\
\hline Scale Factor for Light Flavor & -0.0 & +0.0 & +0.0 & -0.0 \\
\hline Magnet Polarity Modeling & +0.2 & -0.2 & +0.2 & -0.2 \\
\hline Total & & & +0.5 & -0.5 \\
\hline
\end{tabular}

Table D.2 Absolute systematic uncertainties on $A_{\mathrm{FB}}^{\text {lep,pred }}$. The 2nd and 3rd columns list the effect of each systematic variation on $A_{\mathrm{FB}}^{\text {lep,pred }}$, and the 4 th and 5th columns list the resulting up and down uncertainties. 


\section{D.0.3 Systematic uncertainties on reconstruction level asym- metries}

\begin{tabular}{|c|c|c|c|c|}
\hline Source & $\Delta^{+}($in $\%$ & $\overline{\Delta^{-}(\text {in } \%)}$ & $\sigma^{+}($in $\%)$ & $\sigma^{-}($in $\%)$ \\
\hline Jet Efficiency & \multicolumn{2}{|c|}{+0.2} & +0.2 & -0.2 \\
\hline Alternative signal model & \multicolumn{2}{|c|}{+0.5} & +0.5 & -0.5 \\
\hline Vertex Confirmation & \multicolumn{2}{|c|}{-0.2} & +0.2 & -0.2 \\
\hline Lumi & \multicolumn{2}{|c|}{-0.4} & +0.4 & -0.4 \\
\hline Jet Shifting On & \multicolumn{2}{|c|}{-0.1} & +0.1 & -0.1 \\
\hline W+Jets Heavy Flavor & -0.1 & +0.1 & +0.1 & -0.1 \\
\hline W+jets Asymmetry & +0.5 & -0.7 & +0.5 & -0.7 \\
\hline Fake Lepton Selection Rate & -0.1 & +0.1 & +0.1 & -0.1 \\
\hline True Lepton Selection Rate & -0.0 & +0.0 & +0.0 & -0.0 \\
\hline Jet Enetry Resolution & -0.4 & -0.2 & +0.0 & -0.4 \\
\hline Jet Energy Scale & -0.1 & -0.2 & +0.0 & -0.2 \\
\hline Scale Factor for Heavy Flavor & -0.1 & +0.1 & +0.1 & -0.1 \\
\hline Scale Factor for Light Flavor & +0.0 & -0.0 & +0.0 & -0.0 \\
\hline Fitting procedure & \multicolumn{2}{|c|}{ \pm 0.2} & -0.2 & +0.2 \\
\hline Total & & & +0.8 & -1.0 \\
\hline
\end{tabular}

Table D.3 Absolute systematic uncertainties on the observed $A_{\mathrm{FB}}$. The 2nd and 3 rd columns list the effect of each systematic variation on $A_{\mathrm{FB}}$, and the 4 th and 5th columns list the resulting up and down uncertainties. 


\begin{tabular}{|c|c|c|c|c|}
\hline Source & $\Delta^{+}($in $\%)$ & $\Delta^{-}($in $\%)$ & $\sigma^{+}($in $\%)$ & $\sigma^{-}($in $\%)$ \\
\hline Jet Efficiency & \multicolumn{2}{|c|}{+0.1} & +0.1 & -0.1 \\
\hline Vertex Confirmation & \multicolumn{2}{|c|}{-0.1} & +0.1 & -0.1 \\
\hline Lumi & \multicolumn{2}{|c|}{+0.0} & +0.0 & -0.0 \\
\hline Jet Shifting On & \multicolumn{2}{|c|}{-0.0} & +0.0 & -0.0 \\
\hline W+Jets Heavy Flavor & +0.0 & -0.0 & +0.0 & -0.0 \\
\hline W+jets Asymmetry & +0.5 & -0.4 & +0.5 & -0.4 \\
\hline Fake Lepton Selection Rate & +0.3 & -0.3 & +0.3 & -0.3 \\
\hline True Lepton Selection Rate & -0.0 & +0.0 & +0.0 & -0.0 \\
\hline Jet Enetry Resolution & -0.3 & +0.0 & +0.0 & -0.3 \\
\hline Jet Energy Scale & -0.2 & -0.1 & +0.0 & -0.2 \\
\hline Scale Factor for Heavy Flavor & +0.0 & +0.0 & +0.0 & -0.0 \\
\hline Scale Factor for Light Flavor & +0.0 & -0.0 & +0.0 & -0.0 \\
\hline Fitting procedure & \multicolumn{2}{|c|}{ \pm 0.2} & -0.2 & +0.2 \\
\hline Total & & & +0.6 & -0.7 \\
\hline
\end{tabular}

Table D.4 Absolute systematic uncertainties on the observed $A_{\mathrm{FB}}^{l}$. The 2nd and 3rd columns list the effect of each systematic variation on $A_{\mathrm{FB}}^{l}$, and the 4 th and 5th columns list the resulting up and down uncertainties. 


\section{D.0.4 Systematic uncertainties on unfolded asymmetries}

\begin{tabular}{|l|c|c|c|c|}
\hline Source & $\Delta^{+}$(in \%) & $\Delta^{-}$(in $\left.\%\right)$ & $\sigma^{+}$(in \%) & $\sigma^{-}$(in \%) \\
\hline Jet Efficiency & \multicolumn{2}{|c|}{-0.2} & +0.2 & -0.2 \\
Vertex Confirmation & \multicolumn{2}{|c|}{+0.7} & +0.7 & -0.7 \\
Lumi & \multicolumn{2}{|c|}{-0.3} & +0.3 & -0.3 \\
Jet Shifting On & \multicolumn{2}{|c|}{-0.8} & +0.8 & -0.8 \\
W+Jets Heavy Flavor & -0.2 & +0.1 & +0.1 & -0.2 \\
W+jets Asymmetry & -0.0 & -0.0 & +0.0 & -0.0 \\
Fake Lepton Selection Rate & -0.1 & +0.1 & +0.1 & -0.1 \\
True Lepton Selection Rate & +0.0 & +0.0 & +0.0 & -0.0 \\
Jet Energy Resolution & -0.9 & -0.8 & +0.0 & -0.9 \\
Jet Energy Scale & -0.9 & -0.6 & +0.0 & -0.9 \\
Scale Factor for Heavy Flavor & -0.1 & +0.1 & +0.1 & -0.1 \\
Scale Factor for Light Flavor & -0.1 & +0.1 & +0.1 & -0.1 \\
Charge ID & -0.1 & +0.2 & +0.2 & -0.1 \\
Fitted $t \bar{t}$ & +0.6 & -0.6 & +0.6 & -0.6 \\
Fitted $W+$ jets & -0.4 & +0.4 & +0.4 & -0.4 \\
Calibration & -1.0 & +1.1 & +1.1 & -1.0 \\
Acceptance Modeling & +0.0 & -1.6 & +0.0 & -1.6 \\
\hline Total & \multicolumn{4}{|r|}{} \\
\hline
\end{tabular}

Table D.5 Absolute systematic uncertainties on the $50 \rightarrow 26$ unfolded $A_{\mathrm{FB}}$. The 2nd and 3rd columns list the effect of each systematic variation on the unfolded $A_{\mathrm{FB}}$, and the 4 th and 5 th columns list the resulting up and down uncertainties. 


\begin{tabular}{|c|c|c|c|c|}
\hline Source & $\Delta^{+}($in $\%)$ & $\Delta^{-}($in $\%)$ & $\sigma^{+}($in $\%)$ & $\overline{\sigma^{-}}($in $\%)$ \\
\hline Jet Efficiency & \multicolumn{2}{|c|}{-0.1} & +0.1 & -0.1 \\
\hline Vertex Confirmation & \multicolumn{2}{|c|}{-0.8} & +0.8 & -0.8 \\
\hline Lumi & \multicolumn{2}{|c|}{+0.1} & +0.1 & -0.1 \\
\hline Jet Shifting On & \multicolumn{2}{|c|}{+0.1} & +0.1 & -0.1 \\
\hline W+Jets Heavy Flavor & -0.0 & +0.0 & +0.0 & -0.0 \\
\hline W+jets Asymmetry & +0.0 & +0.0 & +0.0 & -0.0 \\
\hline Fake Lepton Selection Rate & +0.4 & -0.3 & +0.4 & -0.3 \\
\hline True Lepton Selection Rate & +0.0 & +0.0 & +0.0 & -0.0 \\
\hline Jet Enetry Resolution & -0.5 & +0.1 & +0.1 & -0.5 \\
\hline Jet Energy Scale & -0.3 & -0.2 & +0.0 & -0.3 \\
\hline Scale Factor for Heavy Flavor & +0.1 & -0.0 & +0.1 & -0.0 \\
\hline Scale Factor for Light Flavor & +0.0 & -0.0 & +0.0 & -0.0 \\
\hline Charge ID & -0.0 & +0.2 & +0.2 & -0.0 \\
\hline fitted ttbar & +0.1 & -0.0 & +0.1 & -0.0 \\
\hline fitted $\mathrm{W}+$ jets & +0.5 & -0.5 & +0.5 & -0.5 \\
\hline Magnet Polarity Modeling & +0.2 & -0.2 & +0.2 & -0.2 \\
\hline Acceptance Modeling & +0.0 & -0.6 & +0.0 & -0.6 \\
\hline Total & & & +1.1 & -1.3 \\
\hline
\end{tabular}

Table D.6 Absolute systematic uncertainties on the unfolded $A_{\mathrm{FB}}^{l}$. The 2nd and 3rd columns list the effect of each systematic variation on the unfolded $A_{\mathrm{FB}}^{l}$, and the 4 th and 5 th columns list the resulting up and down uncertainties. 